
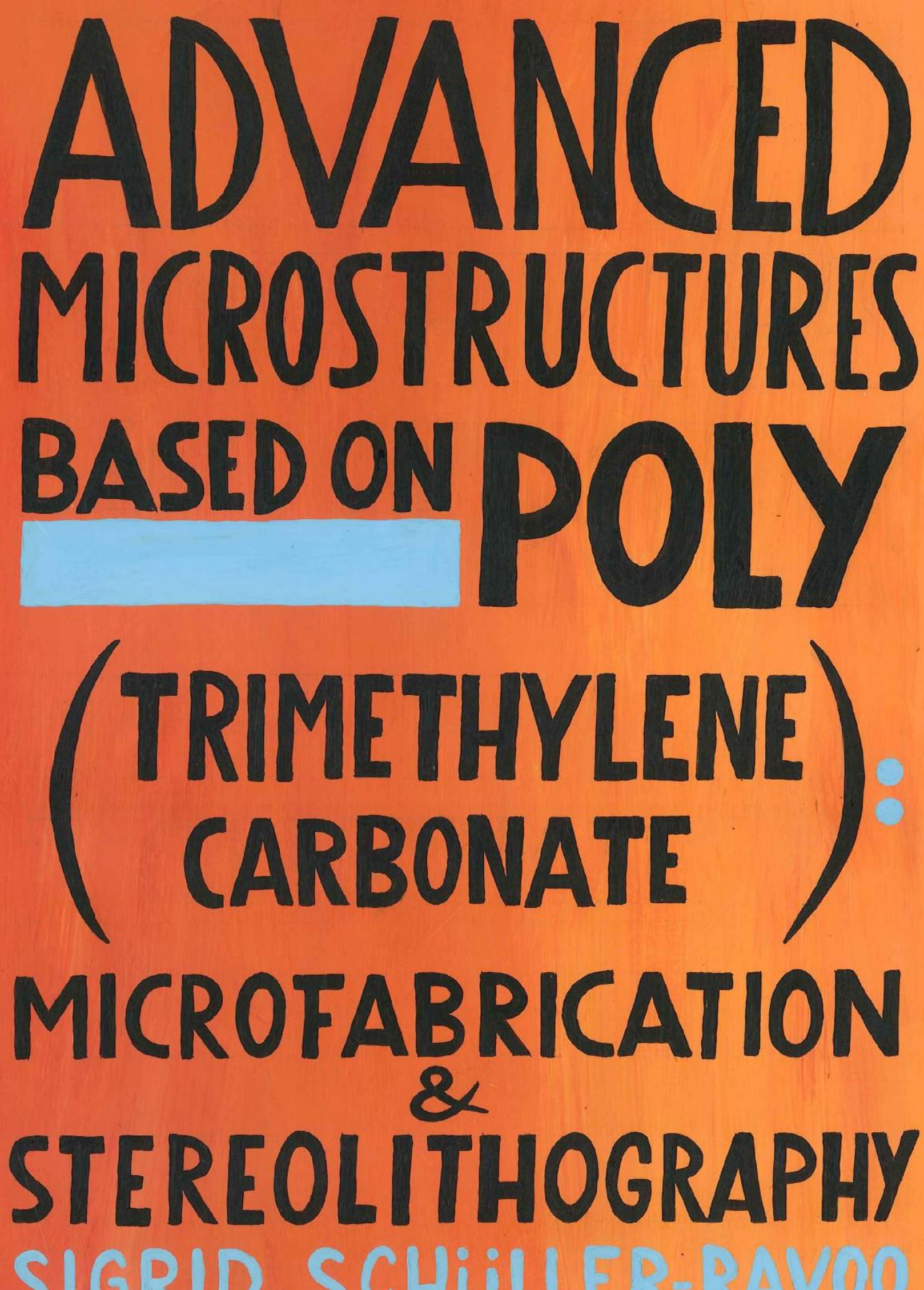


\author{
ADVANCED MICROSTRUCTURES \\ BASED ON \\ POLY(TRIMETHYLENE CARBONATE): \\ MICROFABRICATION AND STEREOLITHOGRAPHY
}

Sigrid Schüller-Ravoo 
The research described in this thesis was carried out between 2005 and 2010 in the research group Polymer Chemistry and Biomaterials at the Institute for Biomedical Technology and Technical Medicine (MIRA), University of Twente, Enschede, The Netherlands. The research was financially supported by the program: "Advanced Polymeric Microstructures for Tissue Engineering" spearhead program of the University of Twente.

Advanced Microstructures Based on Poly(Trimethylene Carbonate):

Microfabrication and Stereolithography

Sigrid Schüller-Ravoo

$\mathrm{PhD}$ Thesis with references and summaries in English and Dutch

University of Twente, Enschede, The Netherlands

ISBN: 978-90-365-3219-8

Printed by Wöhrmann Print Service, Zutphen, The Netherlands

(C) 2011 by Sigrid Schüller-Ravoo, all rights reserved.

Cover Design by Ingeborg Panjer.

(http://www.ingeborgpanjer.nl/)

The printing of this thesis is supported by: 


\title{
ADVANCED MICROSTRUCTURES \\ BASED ON \\ POLY(TRIMETHYLENE CARBONATE): \\ MICROFABRICATION AND STEREOLITHOGRAPHY
}

\section{DISSERTATION}

\author{
to obtain \\ the degree of doctor at the University of Twente, \\ on the authority of the rector magnificus, \\ prof. dr. H. Brinksma, \\ on account of the decision of the graduation committee, \\ to be publicly defended \\ on Friday the $8^{\text {th }}$ July 2011 at $15: 00$
}

by

\section{Sigrid Schüller-Ravoo}

born on the $15^{\text {th }}$ May 1977

in Sibiu, Romania 
This dissertation has been approved by the promoters:

Prof. Dr. Jan Feijen

Prof. Dr. Dirk W. Grijpma

(C) 2011 Sigrid Schüller-Ravoo

ISBN: 978-90-365-3219-8 
To my family and friends

who always believed in me. 



\section{Table of Contents}

$\begin{array}{lll}\text { Chapter } 1 \text { General Introduction } & 3\end{array}$

$\begin{array}{lll}\text { Chapter } 2 & \text { Microstructures for Biomedical Applications and } & 7\end{array}$

Tissue Engineering

Chapter 3 Flexible, Elastic and Tear-Resistant Networks Prepared by

Photo-Crosslinking Poly(Trimethylene Carbonate) Macromers

Chapter 4 Microstructured Photo-Crosslinked Poly(Trimethylene Carbonate)

for Use in Soft Lithography Applications: Biodegradable Structures as Alternative for Poly(Dimethylsiloxane)

Chapter 5 Preparation of Flexible and Elastic Poly(Trimethylene Carbonate) Structures by Stereolithography

Chapter 6 Designed Scaffolds for Cartilage Tissue Engineering Prepared by Stereolithography Using Poly(Trimethylene Carbonate)-Based Resins

Chapter 7 Preparation of a Designed Poly(Trimethylene Carbonate)

Microvascular Network by Stereolithography

Summary

Samenvatting 


\section{Chapter 1}

\section{General Introduction}

We live in a world where knowledge and technology rapidly develop and more and more becomes possible, also in medicine. People suffering from diseased organs or damaged tissues can be helped by transplantations, which allows them a relatively normal life. Unfortunately, the number of available donor tissues and organs does not match the continuously increasing demand.

Biomedical engineering aims to apply knowledge, technology and engineering solutions to solve problems in medicine. Tissue engineering is an approach that could solve the problem of donor shortage. Formulated approximately 20 years ago, tissue engineering combines principles of engineering and life sciences to develop new biological tissues to reconstruct the human body. ${ }^{[1-2]}$ The main approach to engineering tissues requires a scaffold, on which cells (preferably autologous) are seeded and cultured to build up biological tissue. Eventually, the scaffold should degrade and resorb, leaving a fully regenerated, native tissue or organ behind. To make this possible, scientists of different fields must work closely together. Chemists, for example, develop and produce new materials which are used by biologists to grow cells on. Engineers are necessary to develop techniques and instruments to process these materials in an easy, rapid and reproducible way.

Microstructures produced by microfabrication techniques, allow the investigation of cell-cell and cell-material interactions. ${ }^{[3-6]}$ The results of this research has provided useful knowledge that can be applied to tissue engineering. However, tissue engineering poses another challenge: scaffolds need to be engineered in three dimensions. Although scaffolds can be prepared by conventional techniques such as porogen leaching, gas foaming and phase-separation followed by freeze-drying, these methods lead to isotropically distributed pores and pore interconnectivities, made in a not very reproducible manner. ${ }^{[7]}$ Stacking microstructured layers obtained by microfabrication is a way to obtain three-dimensional scaffolds. ${ }^{[8-10]}$ However, although this approach allows better control of parameters such as 
pore size and pore interconnectivity, it is very difficult to reproducibly prepare structures as well.

Scaffolds can reproducibly be prepared by rapid prototyping methods. This term comprises several processing technologies which create three-dimensional structures in an additive (layer-by-layer) manner. Stereolithography is a form of prototyping that has been shown to be very versatile with highest accuracy and precision. ${ }^{[11]}$ Stereolithography is based on the layer-by-layer solidification of a liquid resin by photo-crosslinking according to the (computer-)design of a three-dimensional structure.

Most resins processed by stereolithography yield rigid and stiff materials, and only few of them are biodegradable. ${ }^{[12-17]}$ As numerous tissues and organs of the human body are soft, there is an urgent need for resins that can be processed by stereolithography to yield structures that are flexible and elastic, but also mechanically strong.

Poly(trimethylene carbonate) (PTMC) is an amorphous polymer with a glass transition temperature around $-19{ }^{\circ} \mathrm{C}$. PTMC is a flexible polymer with exceptional mechanical properties at molecular weights above approximately $200000 \mathrm{~g} / \mathrm{mol}$. Cross-linking of this linear polymer by gamma-irradiation increases its creep resistance, while the elastic modulus and tensile strength are not significantly affected. ${ }^{[18]}$ Since it is also biodegradable, it complies with most requirements for soft tissue engineering. Creating simple twodimensional and more complex three-dimensional scaffolds from PTMC by microfabrication and stereolithography would provide new opportunities in the biomedical field, especially in the engineering of soft tissue.

\section{Aims and Outline of this Thesis}

This thesis aims at developing photo-crosslinkable resins based on PTMC macromers for processing by microfabrication techniques and stereolithography. The obtained two- and three-dimensional structures are characterized with regard to their processability, and (biomedically) relevant material properties such as their mechanical performance and compatibility with different cell types.

Chapter 2 introduces basic knowledge on techniques used in this research and on PTMC, the material of choice. Microfabrication techniques for the creation of microstructures, in particular by soft lithography, are described. With regard to the fabrication of threedimensional structures, the principle of stereolithography is briefly explained. Each section contains examples that are relevant to biomedical applications. 
In Chapter 3 PTMC macromers of different molecular weights were synthesized and crosslinked in the melt. It is shown that the thermal and mechanical properties of the resulting networks depend strongly on the macromer molecular weight. Special attention is paid to the toughness and tearing behavior of the networks. This is characterized by the tear propagation strength and the suture retention strength.

Chapter 4 demonstrates the potential application of PTMC macromers in the field of microfabrication as a biodegradable alternative to PDMS. Macromers based on PTMC are processed by casting or hot embossing, and subsequently photo-crosslinking to yield microstructured PTMC surfaces. The obtained surfaces are evaluated for use in two possible applications: as a stamp in microcontact printing and as a structured substrate for smooth muscle cell culturing.

In Chapter 5 PTMC resins based on PTMC macromers of different molecular weights are formulated for application in stereolithography. It is shown that resins based on PTMC macromers with molecular weights below $20150 \mathrm{~g} / \mathrm{mol}$ can successfully be processed into solid and porous three-dimensional structures, using propylene carbonate as a non-reactive diluent. The extracted and dried structures are characterized by their compression moduli. Thermal and tensile properties of solid films prepared by stereolithography are compared to the results obtained in Chapter 3.

Chapter 6 investigates the applicability of three-dimensional porous PTMC structures as scaffolds for cartilage tissue engineering. Highly interconnected structures with a gyroid pore network architecture were seeded with bovine chondrocytes, which were cultured for 6 weeks. During this culturing period the compression moduli of the constructs were determined.

In Chapter 7 we present a three-dimensional design of a microvascular network for use in tissue engineering. The advanced structure is prepared by applying a non-diluted PTMC resin in stereolithography and is characterized by micro-computed tomography, scanning electron microscopy and perfusion experiments. To assess their compatibility with cells, human umbilical vein endothelial cells were cultured on flat disks of the photo-crosslinked polymer. 


\section{References}

[1] Langer R, Vacanti JP. Tissue engineering. Science 1993, 260, 920-926.

[2] Williams DF. On the nature of biomaterials. Biomaterials 2009, 30, 5897-5909.

[3] Bhatia SN, Chen CS. Tissue engineering at the micro-scale. Biomed Microdevices 1999, 2, 131-144.

[4] Folch A, Toner M. Microengineering of cellular interactions. Annu Rev Biomed Eng 2000, 2, 227-256.

[5] Li N, Tourovskaia A, Folch A. Biology on a chip: microfabrication for studying the behaviour of cultured cells. Crit Rev Biomed Eng 2003, 31, 423-488.

[6] Kaji H, Camci-Unal G, Langer R, Khademhosseini A. Engineering systems for the generation of patterned co-cultures for controlling cell-cell interactions. Biochim Biophys Acta 2011, 1810, 239-250.

[7] Muschler GF, Nakamoto C, Griffith LG. Engineering principles of clinical cell-based tissue engineering. J Bone Joint Surg Am 2004, 86, 1541-1558.

[8] Borenstein JT, Weinberg EJ, Orrick BK, Sundback C, Kaazempur-Mofrad MR, Vacanti JP. Microfabrication of three-dimensional engineered scaffolds. Tissue Eng 2007, 13, 1837-1844.

[9] Tan W, Desai TA. Microscale multilayer cocultures for biomimetic blood vessels. $J$ Biomed Mater Res A 2005, 72A, 146-160.

[10] Tan W, Desai TA. Layer-by-layer microfluidics for biomimetic three-dimensional structures. Biomaterials 2004, 25, 1355-1364.

[11] Savalani MM, Harris RA. Layer manufacturing for in vivo devices. P I Mech Eng H 2006, 220, 505-520.

[12] Matsuda T, Mizutani M, Arnold SC. Molecular design of photocurable liquid biodegradable copolymers. 1. Synthesis and photocuring characteristics. Macromolecules 2000, 33, 795-800.

[13] Matsuda T, Mizutani M. Liquid acrylate-endcapped biodegradable poly(epsiloncaprolactone-co-trimethylene carbonate). II. Computer-aided stereolithographic microarchitectural surface photoconstructs. J Biomed Mater Res 2002, 62, 395-403.

[14] Cooke MN, Fisher JP, Dean D, Rimnac C, Mikos AG. Use of stereolithography to manufacture critical-sized 3D biodegradable scaffolds for bone ingrowth. J Biomed Mater Res A 2003, 64B, 65-69.

[15] Lee SJ, Kang HW, Park JK, Rhie JW, Hahn SK, Cho DW. Application of microstereolithography in the development of three-dimensional cartilage regeneration scaffolds. Biomed Microdevices 2008, 10, 233-241.

[16] Jansen J, Melchels FPW, Grijpma DW, Feijen J. Fumaric acid monoethyl esterfunctionalized poly(D,L-lactide)/N-vinyl-2-pyrrolidone resins for the preparation of tissue engineering scaffolds by stereolithography. Biomacromolecules 2009, 10, 214220.

[17] Melchels FPW, Feijen J, Grijpma DW. A poly(D,L-lactide) resin for the preparation of tissue engineering scaffolds by stereolithography. Biomaterials 2009, 30, 38013809.

[18] Pego AP, Grijpma DW, Feijen J. Enhanced mechanical properties of 1,3-trimethylene carbonate polymers and networks. Polymer 2003, 44, 6495-6504. 


\section{Chapter 2}

\section{Microstructures for Biomedical Applications and Tissue Engineering}

Cells are the elementary units of every living organism. They typically have diameters in the order of $10 \mu \mathrm{m}$ and respond to environmental features at micrometer to nanometer length scales. ${ }^{[1]}$ Microstructures are increasingly being investigated for applications in biology and medicine, as their size scale matches the physical dimensions of most microorganisms and individual cells of complex organisms. ${ }^{[2-3]}$ By application of microfabrication techniques in cell culturing studies, scientists have investigated cell-cell and cell-material interactions. ${ }^{[4-7]}$ In recent years, microfabrication techniques have contributed to the development and successful application of microchips in biology and in the clinic. ${ }^{[8]}$ Soft lithography microfabrication techniques allow the production of microstructures and increasingly more so of nanostructures. However, microfabrication is a two-dimensional structure fabrication technique. ${ }^{[9]}$ In certain applications (such as in tissue engineering) three-dimensional structures are required which can only be prepared indirectly when microfabrication techniques are employed (see below).

One of the ultimate goals in biomedicine is the replacement or the regeneration of diseased or damaged tissues and organs. Tissue engineering involves the use of cells that are seeded and cultured on synthetic microstructured scaffolds. To mimic nature as closely as possible, these scaffolds should be three-dimensional. New techniques such as stereolithography are being investigated for the fabrication of advanced three-dimensional microstructured scaffolds for application in tissue engineering. Cells not only respond to the microstructure, but also to the mechanical and chemical properties of the material from which the structure is made. It should therefore be emphasized that the design of tailor-made materials will be a key factor in determining a suitable microstructured surface or scaffold. 


\section{Microfabrication}

Microfabrication is the manufacturing of structures with small-sized features and has an ever increasing impact in science and technology. ${ }^{[10]}$ It provides basic experimental techniques that allow the examination of cells and microorganisms in well-defined and controlled environments. ${ }^{[3]}$ In biomedical research, the use of microstructured devices allows performing thousands of parallel experiments with cells under identical conditions. Using microchannels, small amounts of fluids can be transported, mixed and delivered to cells. $^{[3]}$

A

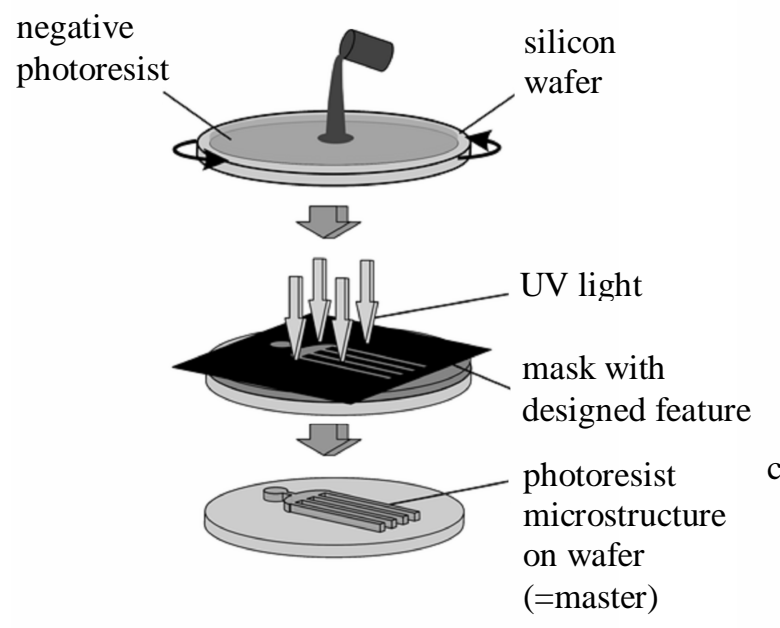

B

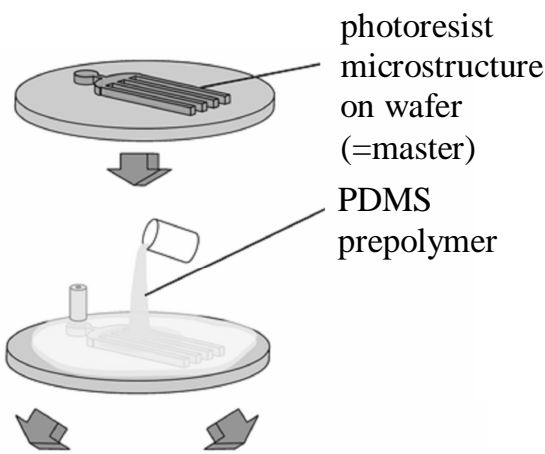

C

D

cured PDMS stamp

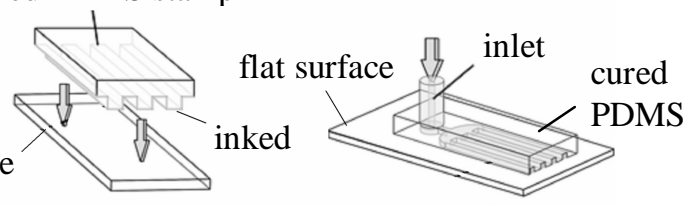

microcontact printing microfluidic device

Figure 1. Preparation of a microstructured silicon wafer by photolithography (A). Soft lithography: Replica molding of PDMS to obtain microstructured PDMS (B) to be used as stamp in micro-contact printing $(C)$ or for the preparation of a microfluidic device $(D)$. (Figure is adapted from reference 6).

Until the end of the last century, microstructured surfaces were mainly created by techniques such as photolithography. ${ }^{[11,14]}$ Here, by use of a mask and photo-crosslinking, a geometric pattern is transferred to a wafer spin-coated with a photoresist (Figure 1A). In the biomedical field these technologies have led to the development of e.g. microfluidics-based biological systems, or high-density thin-film cochlear electrode arrays. ${ }^{[12-13]}$ There are some drawbacks to the application of photolithography: it has to be performed in a clean room facility, maintenance and operation costs for fabrication equipment are very high, it only 
allows the patterning of planar surfaces, and it is limited to a small number of photoresist materials. ${ }^{[8,10]}$

\section{Soft Lithography}

Soft lithography was introduced by the group of Whitesides as an alternative microfabrication method to photolithography. ${ }^{[10]}$ Soft lithography techniques comprise microfabrication methods in which a patterned elastomer is prepared. These key elements are then used as stamps or molds to transfer a pattern to another substrate. Important soft lithography techniques include replica molding (REM) (Figure 1B), (hot) embossing (Figure 2) and microcontact printing $(\mu \mathrm{CP})$ (Figure 1C). ${ }^{[14]}$

\section{Use of Poly(dimethylsiloxane) in Soft Lithography}

Poly(dimethylsiloxane) (PDMS) has emerged as the material of choice in soft lithography, and it is used as stamps, masks, molds for substrates for microfluidic devices. The properties of PDMS make it well-suited or many applications, and (perhaps even more importantly) it is well-known and readily available commercially. Advantageous properties of PDMS are its flexibility and elasticity. The elastomer conforms well to non-planar surfaces and is easily released from complex molds, which allows the preparation of highquality patterns and structures. It is chemically inert and has low interfacial free energy with air. Furthermore, it is homogeneous, isotropic and optically transparent, allowing UV crosslinking of resins. The material is quite durable, and can be used in stamping processes for long periods of time without noticeable changes in performance. ${ }^{[14]}$ Regarding cell culturing experiments, PDMS shows relatively good permeability to non-polar gases such as $\mathrm{O}_{2}, \mathrm{~N}_{2}$ and $\mathrm{CO}_{2}$ which is crucial when the material is used in microfluidic cell culturing channel systems. $^{[15]}$

There are, however, some important shortcomings to the use of PDMS in soft lithography. When printing small features by $\mu \mathrm{CP}$, for example, the low E-modulus of the PDMS material (approximately $1.5 \mathrm{MPa}$ ) results in collapse or sagging of the stamp when contacting the substrate surface and limits the achievable resolution. ${ }^{[16]}$ Also, the hydrophobic nature of PDMS makes it less suitable for the printing of polar molecules like proteins or DNA. Furthermore, PDMS was found to absorb hydrophobic small 
molecules. ${ }^{[17]}$ When microfluidic devices made of PDMS are used in cell culture, drug discovery or proteomic analysis, it is necessary to take this absorption into account. As the components of interest are only present in micro- or nanomolar concentrations, the composition of the solutions that are being investigated might change significantly. Another limitation of PDMS concerns its application as an implantable material or as a tissue engineering scaffolding structure: PDMS is highly resistant to degradation and in long term culture shows limited compatibility with cells. ${ }^{[18-21]}$ With regard to its mechanical properties, it should be realized that when not reinforced, which is mostly the case in microfabrication applications, PDMS is a brittle material with poor resistance to tearing. ${ }^{[22]}$ Although PDMS is not seen as part of a successful path to product manufacturing and development in industry, it is an excellent material for prototyping approaches and researchers will continue working with it. ${ }^{[23]}$

\section{Replica Molding}

Replica molding (REM) is a process that allows the replication of (micro)structures from a master to another material. The material to be structured is cast on the surface of a structured master and solidified by heat or (UV) light-irradiation. The structured master can be a silicon wafer or another hard material, in soft lithography applications it is often a PDMS structure. ${ }^{[3,25]}$ PDMS masters (obtained by REM using a silicon wafer, see Figure 1B) are advantageous because of their flexibility and low interfacial free energy, which allows easy detachment of replicated materials. ${ }^{[10,24]}$ REM is often used to fabricate microfluidic devices. ${ }^{[25]}$

Other molding processes in soft lithography also use PDMS molds prepared by REM. ${ }^{[14]}$ Using microstructured PDMS as a mold, several biodegradable materials have been structured. These include poly(DL-lactic-co-glycolide), poly( $\varepsilon$-caprolactone-DL-lactide) tetra-acrylate and silk fibroin. ${ }^{[26-29]}$

\section{Hot Embossing}

In hot embossing, a microstructure is created by imprinting the structure of a master onto a polymer that is softened by heat (Figure 2). ${ }^{[14]}$ A thermoplastic polymer film is placed on top of a master in a molding machine, and pressure is applied after heating to above the glass-transition temperature of the polymer. The polymer forms to the relief structure of the 
master, and an inverted replicate of the microstructure is created. ${ }^{[30]}$ In conventional hot embossing, the polymer film is then cooled and the replicated structure can be demolded from the master. Hot embossing can also be used to prepare microstructured thermosetting polymers: a polymer resin is then cured by e.g. photo-crosslinking with UV light. ${ }^{[31-32]}$ Since hot embossing provides microstructured surfaces with high precision, the technique is frequently applied in the fabrication of optical components such as optical waveguides. ${ }^{[32]}$ Hot embossing is a cost-effective, high-throughput process that is well suited for mass production. $^{[14,30]}$

$$
\mathrm{T}=\text { room temperature } \quad \mathrm{T}=\text { room temperature }
$$

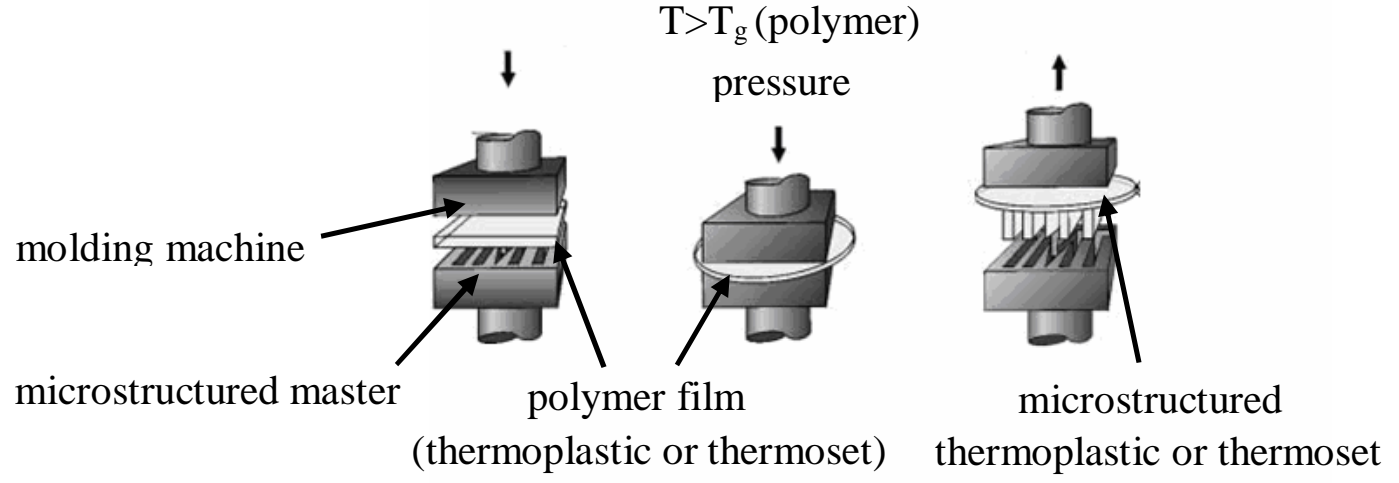

Figure 2. Hot embossing: preparation of a microstructured thermoplastic or thermoset. (Figure is adapted from reference 30 ).

\section{Microcontact Printing}

Microcontact printing produces micropatterns and microstructures on the surface of a substrate according to the relief pattern of the stamp using self-assembly. ${ }^{[14]}$ The technique was first developed to transfer thiol-patterns onto gold surfaces, but is now being used to transfer a variety of inks. These include aqueous solutions of proteins and other biomolecules. ${ }^{[33-35]}$ The range of substrate materials that is being patterned has significantly expanded and includes glass, silicon and polymers like poly(styrene) (PS), poly(lactid acid) (PLA) and poly(methyl methacrylate) (PMMA). ${ }^{[16,36]}$ Due to its many advantageous properties with regard to $\mu \mathrm{CP}$, (see above), PDMS is still the most widely used stamp material, although other polymers and hydrogels are being investigated as alternative materials. ${ }^{[16,37]}$

Microcontact printing has successfully been used in the biomedical field. In 1998 Bernard et al. were the first to transfer proteins by $\mu \mathrm{CP}$ using (PDMS) stamps to a variety of 
different substrates such as glass, silicon and PS. ${ }^{[38]}$ To spatially control the organization, spreading, morphology and alignment of cells for tissue engineering applications, cells and proteins have been patterned on chitosan, poly(DL-lactic-co-glycolic acid) (PLGA) and PLA. ${ }^{[39-40]}$ In the development of implantable nerve guides, microcontact printing has been used to guide Schwann cells and nerve cells on patterned substrates. ${ }^{[41]}$ Micropatterned arrays on PLGA and on poly(ethylene glycol) and poly(DL-lactic acid) copolymer (PEG/PLA) surfaces were used to study retinal pigment epithelial cell attachment and morphology. ${ }^{[42]}$ For the fabrication of microreactor arrays, avidin was printed on activated poly(ethylene terephthalate) surfaces to form specific patterns that allow the selective attachment of polyelectrolyte microcapsules functionalized with biotin. The formed capsule arrays are of great interest for drug release and targeting investigations. ${ }^{[43]}$ Microcontact printing has also been used to obtain polymerized hydrogel microstructures for application in biomedical microdevices. ${ }^{[44]}$

\section{Microfluidic Devices}

Microfluidic devices are prepared by reversibly or irreversibly sealing a microstructured surface containing channels of approximately $5 \mu \mathrm{m}$ to $500 \mu \mathrm{m}$ against a flat surface (see Figure 1D). ${ }^{[3]}$ The flat surface can be PDMS or another material such as glass, while the structured surface is most often prepared from PDMS. ${ }^{[25]}$ Microfluidic systems were developed to process or manipulate fluids and were first used in analytical applications. ${ }^{[45]}$ They were introduced into the field of molecular biology in the 1980s, when the interest in genomics and other microanalytic fields related to molecular biology like high-throughput DNA sequencing was at its height. Microfluidics offered approaches to increase the sensitivity, resolution and the throughput of the analytical methods needed. ${ }^{[45]}$

Microfluidic devices have several properties that make them very useful to conduct biological experiments. ${ }^{[3,12]}$ Small fluid flows are precisely controlled at high resolutions, and only small volumes of samples and reagents are required. In microfluidic devices reaction times are short and unit costs are low. Furthermore, microfluidic units can be easily upscaled and arrayed to facilitate high-throughput experimentation. Most microfluidic systems are compatible with imaging and microscopy techniques.

In biomedical engineering, microfluidic systems have been used in bioanalyses, in the development of drugs and in investigating protein crystallization conditions. ${ }^{[46-48]}$ By binding bioactive substrates in microchannels, microfluidic devices with specific patterns 
of bioactive substrates can be obtained. This allows the creation of multi-phenotype cell arrays for high-throughput investigations of cell-biomolecule interactions. ${ }^{[49-50]}$ Microfluidic systems are powerful tools to investigate biological phenomena in vitro and in vivo, as they allow to control the flow of fluids and soluble factors, thereby defining cellcell and cell-material interactions. ${ }^{[51-52]}$ The behavior of cells can be analyzed at high resolutions both on single cell and on multi-cellular level.

The potential of microfluidic biomaterial devices in tissue engineering has been evaluated, especially in the form of branched, microfluidic networks. ${ }^{[53]}$ Many organs in the human body such as the liver, the kidneys and the lungs, can be considered to be microfluidic processors with a vascular network. ${ }^{[54]}$ To successfully engineer an organ, replication of this vascular network is necessary. With this aim in mind, biodegradable microfluidic structures based on poly(D,L-lactic-co-glycolide) have been fabricated. ${ }^{[26]}$

\section{Stereolithography}

Rapid prototyping (RP) techniques use additive processes to create complex three-dimensional constructs in a layer-by-layer manner. They mostly allow only average to good control over microstructures, with resolutions close to $100 \mu \mathrm{m} .{ }^{[55]}$ They are applied in the automotive and in the jewellery industry, but are increasingly being used in biomedical research to manufacture surgical tools, implants, scaffolds and other biomedical devices. ${ }^{[56-}$ ${ }^{57]}$ Stereolithography (SL) is an RP technique based on the spatially controlled solidification of a liquid, photo-crosslinkable resin upon illumination with (laser) light. The technology became commercially available in $1988 .{ }^{[58]}$ It is the most versatile, accurate and precise RP technique, and allows the fabrication of decimeter sized three-dimensional structures at high resolutions with an accuracy of $20 \mu \mathrm{m} .{ }^{[57,59]}$ Two-photon stereolithography, which is based on photo-polymerization by two-photon light absorption, even allows the creation of microstructures with resolutions of approximately $100 \mathrm{~nm} .^{[60]}$

In stereolithography, structures are built with use of a computer-aided design (CAD) file. This file can be a three-dimensional design created with graphical computer software, but it can also be obtained from the data acquired with (clinical) imaging techniques such as magnetic resonance imaging (MRI) and computed tomography (CT). ${ }^{[61]}$ The CAD file contains all necessary information regarding the structure that is to be built (geometry, size, porosity, etc.), and is then converted into standard triangulation language (STL) format. In 
order to build the desired structure in a layer-by-layer manner, the virtual structure is subsequently sliced into layers of $15-100 \mu \mathrm{m}$. The thickness of these slices determines the building resolution in the z-direction, the minimal thickness depends on the capabilities of the stereolithography apparatus. From each slice a corresponding two-dimensional pixel pattern or mask is derived that determines where a photo-crosslinkable resin layer will be illuminated. Only at these pixel positions the layer of resin with given thickness solidifies. This can be done using an array of mirrors such as a digital mirror device. Carefully controlling the light penetration depth allows each layer to be bound to its preceding one, and a three dimensional structure can be created by photo-crosslinking sequential resin layers that are illuminated using different pixel patterns. ${ }^{[62]}$ In Figure 3 a scheme of the stereolithography setup used in the research described in this thesis is presented.

In this manner stereolithography can be used to reproducibly prepare three dimensional tissue engineering scaffolds with well-defined pore network architectures. Structures with defined pore network architectures, precise porosities, pore sizes and pore size distributions, and pore interconnectivities can be designed and built at high resolutions. Even structures with gradients in porosity and pore size can be prepared. ${ }^{[63]}$ With use of clinical imaging data, as mentioned before, customized implants with tailored (micro)structure, shape and size can be built to conform to the defect or site of injury. The method also allows the large scale production of identical constructs for use in drug discovery and fundamental scientific investigations. ${ }^{[64]}$

The number of biocompatible resins that can be processed by stereolithography is very limited, especially when resorbable medical implants or scaffolds are desired. Resins based on poly(propylene fumarate), poly(ethylene glycol), poly(D,L-lactide) and (co)polymers of trimethylene carbonate and $\varepsilon$-caprolactone have been investigated for these purposes. ${ }^{[62,65-72]}$ The use of these resins results in the formation of brittle or rigid networks. Only few stereolithography resins have been developed that yield flexible and elastic networks suitable for soft tissue engineering applications. These resins were based on functionalized poly(D,L-lactide-co- $\varepsilon$-caprolactone), poly(ethylene glycol)dimethacrylate and poly(ethylene oxide). ${ }^{[63,73-74]}$ 


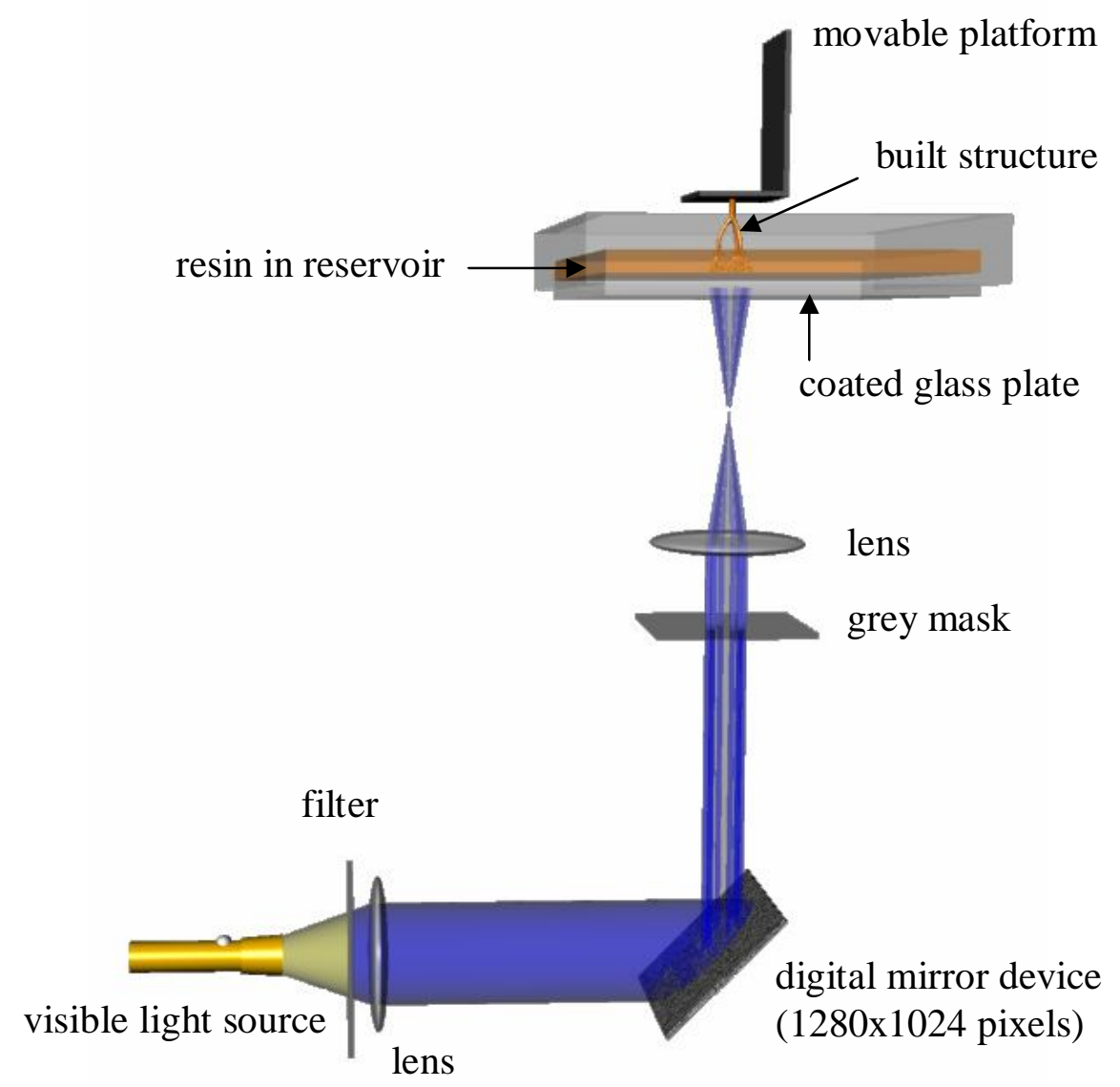

Figure 3. Schematic diagram of a stereolithography setup equipped with a digital mirror device that projects a pixel pattern of visible light on a photocurable resin.

\section{Tissue Engineering}

Every year millions of people suffer from serious health problems related to tissue loss and end-stage organ failure while waiting for a suitable transplant. ${ }^{[75]}$ Tissue engineering could become important in solving the shortage of donor tissues and organs that are available for transplantation. Langer and Vacanti defined tissue engineering as "an interdisciplinary field that applies the principles of engineering and life sciences towards the development of biological substitutes that restore, maintain, or improve tissue function". ${ }^{[75]}$ The approach for the engineering of tissue most often involves the culturing of cells (preferably autologous cells) on a resorbable scaffold. The scaffold functions as a framework with appropriate physical-, chemical- and mechanical properties that allow the cells to penetrate, attach, and proliferate, bringing them in close proximity to each other and allowing them to assemble towards a three-dimensional body-own tissue. The scaffolding structure should 
also ensure adequate oxygen and nutrient levels to the cells and allow the removal of metabolic waste products. It has to show mechanical integrity during formation of the tissue and then preferably degrade and resorb. ${ }^{[76]}$ The degradation products should be non-toxic, low molecular weight compounds that can be excreted via natural pathways.

Porous structures for use as tissue engineering scaffolds have mostly been produced by conventional methods such as salt-leaching, gas-foaming and phase-separation followed by freeze-drying. With these methods, however, control of the pore network characteristics and parameters like porosity and pore size is limited. ${ }^{[77]}$ Microfabrication methods can be of great importance in tissue engineering. Fundamental studies of cell-cell, cell-material and cell-microstructure interactions have played a vital role in understanding biological processes and the basic principles required to successfully engineer tissues. ${ }^{[52,54]}$ Microfabrication techniques allow the preparation of structured material surfaces with which these interactions can be investigated in two dimensions. ${ }^{[9]}$ However, to reproduce in vivo conditions, cell culturing in three dimensions is required. ${ }^{[78]}$ Three-dimensional structures have been obtained by stacking such microstructured layers. ${ }^{[54]}$ Complex systems prepared from various layers of cells and biopolymers have been prepared in this way, but the process is difficult and not very reproducible. ${ }^{[79-80]}$ Rapid prototyping techniques like stereolithography allow a much more controlled way of fabricating scaffolding structures. It is crucial that (micro)structures can be tailor made with minimal limitations to their design and preparation, since the optimal (pore) architecture and scaffold chemistry will depend on the tissue that is to be engineered. ${ }^{[76]}$

Vascularization is essential in tissue engineering; a microvascular network ensures the transport of oxygen and nutrients to the growing cells and the removal of waste products. Most tissue engineered constructs do not have a microvascular network at the time of implantation. ${ }^{[81]}$ While engineered tissues thinner than $2 \mathrm{~mm}$ can survive by diffusion of oxygen and essential nutrients only (the diffusional penetration of oxygen in native tissues is $100-200 \mu \mathrm{m}$ ), this is not sufficient when engineering thicker and more complex tissues like cardiac muscle or liver. ${ }^{[81]}$ For the latter a microvascular system embedded in the scaffold is indispensable to maintain cellular function. ${ }^{[82]}$

Freed et al. envisage that a next generation of scaffolds, based on new scaffold materials and structures will be critical for the future success of engineered tissue replacements. These scaffolds will seamlessly integrate thin-walled, bifurcating microchannels lined with endothelial cells with extravascular compartments containing other cell types, collagen-like 
networks, and systems for controlled release of angiogenic or other growth factors to form a pre-defined template for a tissue vasculature. ${ }^{[81]}$ For clinical applications, the manufacturing process should allow the presence of biological components in certain applications and produce scaffolds in a reproducible, controlled and cost-effective way. ${ }^{[55]}$ It has been stated that "Within the context of tissue engineering and regenerative medicine, there continues to be a clear and present need to develop advanced materials design and processing methods that can better replicate the exquisite architecture and functional properties of native tissues." [81]

\section{Poly(Trimethylene Carbonate)}

Poly(trimethylene carbonate) (PTMC) has been known since $1930 .{ }^{[83]}$ It is an amorphous polymer with low glass transition, and is prepared by ring opening polymerization of the cyclic trimethylene carbonate (TMC) monomer. ${ }^{[84]}$ It can be copolymerized with other cyclic monomers to yield materials with tuneable physical and chemical properties. Upon crosslinking, flexible and elastic (co)polymer networks are obtained.

Materials based on PTMC are very useful in biomedical applications like tissue engineering and drug delivery. ${ }^{\left[{ }^{[5]}\right.}$ Linear (co)polymers and (co)polymer networks prepared from TMC and $\mathrm{D}, \mathrm{L}$-lactide or $\varepsilon$-caprolactone were shown to be compatible with a large number of cells: Schwann cells, human umbilical vein endothelial cells, rat cardiomyocytes, human skin fibroblasts, smooth muscle cells and mouse pre-myoblast $\mathrm{C} 2 \mathrm{C} 12$ cells all showed good cell attachment and proliferation in vitro on the surface of these materials. Implantation experiments in small animals showed only a mild tissue response. ${ }^{[85-88,93,101-102,104]}$

Absorbable sutures prepared from copolymers containing TMC as a comonomer were introduced in 1985. However, at that time, PTMC homopolymers were considered to be less suitable as an implant material due to their supposedly inferior mechanical properties. ${ }^{[89-90]}$ In later work our group showed this not to be necessarily true, as the mechanical properties of the polymer strongly depend on its molecular weight. ${ }^{[91]}$ While number average molecular weights below $50000 \mathrm{~g} / \mathrm{mol}$ lead to gummy materials with poor mechanical properties, very high molecular weight PTMC ( $\overline{\mathrm{M}}_{n}$ above approximately $200000 \mathrm{~g} / \mathrm{mol}$ ) gives a flexible material with excellent properties due to strain-induced crystallization. High molecular weight linear PTMC is an amorphous, tough and flexible solid with a glass transition temperature of $-19{ }^{\circ} \mathrm{C}$, and was used for the preparation of 
porous artificial nerve guides and cardiac tissue engineering scaffolds. ${ }^{[22-93]}$ By comparison, low molecular weight PTMC is a viscous liquid at room temperature and at body temperature, making it a applicable as an injectable polymer for localized drug delivery. ${ }^{[94]}$ The resistance to creep of the flexible polymer significantly increases when it is crosslinked. ${ }^{[91]}$ Such PTMC networks have been prepared by gamma-irradiation of linear high molecular weight polymer or by photo-crosslinking functionalized macromers based on TMC using UV or visible light. ${ }^{[65,95-97]}$

PTMC based polymers and networks degrade by an enzymatic surface erosion mechanism, without the release of acidic degradation products. ${ }^{[65,98-100]}$ In vivo, linear high molecular weight PTMC films were found to degrade in 3 weeks. ${ }^{[99]}$ Upon crosslinking by gamma irradiation doses of 25 to $100 \mathrm{kGy}$, PTMC network films eroded upon subcutaneous implantation in rats within 4 weeks. The erosion rate was independent of irradiation dose. $^{[101]}$ The surface erosion behavior of (crosslinked) PTMC films in vivo could be replicated in vitro using aqueous lipase and cholesterol esterase enzyme solutions. Typically, non-crosslinked PTMC was found to completely erode within about eight weeks, while PTMC networks prepared by gamma-irradiation at 25 and $50 \mathrm{kGy}$, respectively, showed mass losses of approximately 65 and $55 \%$ after the same period. PTMC networks prepared by photo-crosslinking acrylate end-capped PTMC macromers with molecular weight $=7300 \mathrm{~g} / \mathrm{mol}$, showed a mass loss of $33 \%$ after 44 weeks under the same conditions. ${ }^{[95]}$ This implies that these dense networks degrade at even slower rates than the ones obtained by gamma-irradiation. The degradation rates of linear polytrimethylene carbonate polymers and networks can be further tuned by copolymerization of trimethylene carbonate with $\varepsilon$-caprolactone or D,L-lactide. ${ }^{[33,98-99]}$

With regard to applications in tissue engineering, porous structures have been prepared from high molecular weight PTMC. ${ }^{[102-104]}$ By phase separation micromolding, microstructured porous PTMC films with pore sizes between 2-20 $\mu \mathrm{m}$ and porosities of up to $31 \%$ were obtained. ${ }^{[102]}$ However, changes in the morphology of porous structures were observed during cell culturing when linear PTMC materials were used. By crosslinking, the collapse of pores in porous PTMC structures can be avoided. ${ }^{[103]}$ Dimensionally-stable tubular PTMC scaffolds were prepared by gamma-irradiating composites of high molecular weight PTMC and salt or sugar particles, followed by leaching the porogen with water. In this way a flexible and elastic scaffold with an interconnected pore network with average pore sizes of $110 \mu \mathrm{m}$ (salt) or $60 \mu \mathrm{m}$ (sugar) could be obtained. 


\section{Conclusions}

Two-dimensional microstructures created by microfabrication play a significant role in understanding the principles of biology, and can thus be important analytical tools in medicine. However, more complex microstructured three-dimensional structures are often needed as well. In tissue engineering for example, space filling three-dimensional scaffolds are required to mimic the in vivo conditions as closely as possible when cells are cultured, differentiated and expanded to form tissues that replace diseased tissues or organs. Stereolithography is a technique that allows the reproducible manufacturing of designed three-dimensional structures at micrometer scale resolution. In combination with (clinical) imaging techniques, stereolithography is an excellent technique to prepare advanced scaffolds for tissue engineering. The number of resins that can be processed by stereolithography to yield biocompatible and biodegradable materials, especially with flexible and elastic properties, is very limited. Poly(trimethylene carbonate) is known to be such a versatile polymer. It can be concluded that there is a great potential in a variety of biomedical applications for resins based on poly(trimethylene carbonate) macromers in preparing two-dimensional structures by microfabrication and three-dimensional structures by stereolithography.

\section{References}

[1] Stevens MM, George JH. Exploring and engineering the cell surface interface. Science 2005, 310, 1135-1138

[2] Mata A, Fleischman AJ, Roy S. Characterization of polydimethylsiloxane (PDMS) properties for biomedical micro/nanosystems. Biomed Microdevices 2005, 7, 281293.

[3] Weibel DB, DiLuzio WR, Whiteside GM. Microfabrication meets microbiology. Nat Rev Microbiol 2007, 5, 209-218.

[4] Bhatia SN, Chen CS. Tissue engineering at the micro-scale. Biomed Microdevices 1999, 2, 131-144.

[5] Folch A, Toner M. Microengineering of cellular interactions. Annu Rev Biomed Eng 2000, 2, 227-256.

[6] Li N, Tourovskaia A, Folch A. Biology on a chip: microfabrication for studying the behaviour of cultured cells. Crit Rev Biomed Eng 2003, 31, 423-488.

[7] Kaji H, Camci-Unal G, Langer R, Khademhosseini A. Engineering systems for the generation of patterned co-cultures for controlling cell-cell interactions. Biochim Biophys Acta 2011, 1810, 239-250.

[8] Pan T, Wang W. From cleanroom to desktop: emerging micro-nanofabrication technology for biomedical applications. Ann Biomed Eng 2011, 39, 600-620. 
[9] King KR, Wang CCJ, Kaazempur-Mofrad MR, Vacanti JP, Borenstein JT. Biodegradable microfluidics. Adv Mater 2004, 16, 2007-2012.

[10] Xia Y, Whitesides GM. Soft lithography. Angew Chem Int Ed 1998, 37, 550-575.

[11] Brambley D, Martin B, Prewett PD. Microlithography: an overview. Adv Mater Opt Electron 1994, 4, 55-74.

[12] Breslauer DN, Lee PJ, Lee LP. Microfluidics-based systems biology. Mol BioSyst 2006, 2, 97-112.

[13] Wise KD, Bhatti PT, Wang J, Friedrich CR. High-density cochlear implants with position sensing and control. Hearing Res 2008, 242, 22-30.

[14] Xia Y, Whitesides GM. Soft lithography. Annu Rev Mater Sci 1998, 28, 153-184.

[15] Whitesides GM, Ostuni E, Takayama S, Jiang X, Ingber DE. Soft lithography in biology and biochemistry. Annu Rev Biomed Eng 2001, 3, 335-373.

[16] Kaufmann T, Ravoo BJ. Stamps, inks and substrates: polymers in microcontact printing. Polym Chem 2010, 1, 371-387.

[17] Toepke MW, Beebe DJ. PDMS absorption of small molecules and consequences in microfluidic applications. Lab Chip 2006, 6, 1484-1486.

[18] Sarkar S, Dadhania M, Rourke P, Desai TA, Wong JY. Vascular tissue engineering: microtextured scaffold templates to control organization of vascular smooth muscle cells and extracellular matrix. Acta Biomater 2005, 1, 93-100.

[19] Jo B-H, Van Lerberghe LM, Motsegood KM, Beebe DJ. Three-dimensional microchannel fabrication in polydimethylsiloxane (PDMS) elastomer. J Microelectromech $S$ 2000, 9, 76-81.

[20] Bettinger EJ, Weinberg EJ, Kulig KM, Vacanti JP, Wang Y, Borenstein JT, Langer R. Three-dimensional microfluidic tissue engineering scaffolds using a flexible biodegradable polymer. Adv Mater 2006, 18, 165-169.

[21] Moraes C, Kagoma YK, Beca BM, Tonelli-Zasarsky RLM, Sun Y, Simmons CA. Integrating polyurethane culture substrates into poly(dimethylsiloxane) microdevices. Biomaterials 2009, 30, 5241-5250.

[22] Kumudinie C, Mark JE. Tearing energies for in-situ reinforced poly(dimethylsiloxane) networks. Mat Sci Eng C 2000, 11, 61-66.

[23] Mukhopadhyay R. When PDMS isn't the best. Anal Chem 2007, 79, 3248-3253.

[24] Armani DK, Liu C. Microfabrication technology for polycaprolactone, a biodegradable polymer. J Micromech Microeng 2000, 10, 80-84.

[25] McDonald JC, Duffy DC, Anderson JR, Chiu DT, Wu H, Schueller OJA, Whitesides GM. Fabrication of microfluidic systems in poly(dimethylsiloxane). Electrophoresis 2000, 21, 27-40.

[26] King K, Wang C, Kaazempur-Mofrad M, Vacanti J, Borenstein J. Biodegradable microfluidics. Adv Mater 2004, 16, 2007-2012.

[27] Vozzi G, Flaim CJ, Bianchi F, Ahluwalia A, Bhatia S. Microfabricated PLGA scaffolds: a comparative study for application to tissue engineering. Mat Sci Eng C 2002, 20, 43-47.

[28] Leclerc E, Furukawa KS, Miyata F, Sakai Y, Ushida T, Fujii T. Fabrication of microstructures in photosensitive biodegradable polymers for tissue engineering applications. Biomaterials 2004, 25, 4683-4690.

[29] Bettinger CJ, Cyr KM, Matsumoto A, Langer R, Borenstein JF, Kaplan DL. Silk fibroin microfluidic devices. Adv Mater 2007, 19, 2847-2850.

[30] Gerlach A, Knebel G, Guber AE, Heckele M, Herrmann D, Muslija A, Schaller Th. Microfabrication of single-use plastic microfluidic devices for high-throughput screening and DNA analysis. Microsys Technol 2002, 7, 265-268. 
[31] Schuh K, Prucker O, Rühe J. Surface attached polymer networks through thermally induced cross-linking of sulfonyl azide group containing polymers. Macromolecules 2008, 41, 9284-9289.

[32] Choi C-G. Fabrication of optical waveguides in thermosetting polymers using hot embossing. J Micromech Microeng 2004, 14, 945-949.

[33] Kumar A, Whitesides GM. Features of gold having micrometer to centimeter dimensions can be formed through a combination of stamping with an elastomeric stamp and an alkanethiol "ink" followed by chemical etching. Appl Phys Lett 1993, 63, 2002-2004.

[34] Kumar A, Biebuyck HA, Whitesides GM. Patterning self-assembled monolayers: applications in materials science. Langmuir 1994, 10, 1498-1511.

[35] Bernard A, Renault JP, Michel B, Bosshard HR, Delamarche E. Microcontact printing of proteins. Adv Mater 2000, 12, 1067-1070.

[36] Perl A, Reinhoudt DN, Huskens J. Microcontact printing: limitations and achievements. Adv Mater 2009, 21, 2257-2268.

[37] $\mathrm{Xu} \mathrm{H}$, Huskens J. Versatile stamps in microcontact printing: transferring inks by molecular recognition and from ink reservoirs. Chem Eur J 2010, 16, 2342-2348.

[38] Bernard A, Delamarche E, Schmid H, Michel B, Bosshard HR, Biebuyck H. Printing patterns of proteins. Langmuir 1998, 14, 2225-2229.

[39] Kumar G, Wang YC, Co C, Ho C-C. Spatially controlled cell engineering on biomaterials using polyelectrolytes. Langmuir 2003, 19, 10550-10556.

[40] Lin C-C, Co CC, Ho C-C. Micropatterning proteins and cells on polylactic acid and poly(lactide-co-glycolide). Biomaterials 2005, 26, 3655-3662.

[41] Schmalenberg KE, Buettner HM, Uhrich KE. Microcontact printing of proteins on oxygen plasma-activated poly(methyl methacrylate). Biomaterials 2004, 25, 18511857.

[42] Lu L, Nyalakonda K, Kam L, Bizios R, Göpferich A, Mikos AG. Retinal pigment epithelial cell adhesion on novel micropatterned surfaces fabricated from synthetic biodegradable polymers. Biomaterials 2001, 22, 291-297.

[43] Wang B, Zhao Q, Wang F, Gao C. Biologically driven assembly of polyelectrolyte microcapsule patterns to fabricate microreactor arrays. Angew Chem Int Ed 2006, 45, 1560-1563.

[44] Biswal D, Chirra HD, Hilt JZ. Fabrication of hydrogel microstructures using polymerization controlled by microcontact printing (PC mu CP). Biomed Microdevices 2008, 10, 213-219.

[45] Whitesides GM. The origins and the future of microfluidics. Nature 2006, 442, 368373.

[46] Sia SK, Whitesides GM. Microfluidic devices fabricated in poly(dimethylsiloxane) for biological studies. Electrophoresis 2003, 24, 3563-3576.

[47] Pihl J, Karlsson M, Chiu DT. Microfluidic technologies in drug discovery. Drug Discov Today 2005, 10, 1377-1383.

[48] Hansen CL, Skordalakes E, Berger JM, Quake SR. A robust and scalable microfluidic metering method that allows protein crystal growth by free interface diffusion. $P$ Natl Acad Sci USA 2002, 99, 16531-16536.

[49] Khademhosseini A, Yeh J, Eng G, Karp J, Kaji H, Borenstein J, Farokhzad OC, Langer R. Cell docking inside microwells within reversibly sealed microfluidic channels for fabricating multiphenotype cell arrays. Lab Chip 2005, 5, 1380-1386.

[50] Li Y, Yuan B, Ji H, Han D, Chen S, Tian F, Jiang X. A method for patterning multiple types of cells by using electrochemical desorption of self-assembled monolayers within microfluidic channels. Angew Chem Int Ed 2007, 119, 1112-1114. 
[51] Park TH, Shuler ML. Integration of cell culture and microfabrication technology. Biotechnol Prog 2003, 19, 243-253.

[52] Bettinger CJ, Borenstein JT. Biomaterials-based microfluidics for engineered tissue constructs. Soft Matter 2010, 6, 4999-5015.

[53] Andersson H, Van den Berg A. Microfabrication and microfluidics for tissue engineering: state of the art and future opportunities. Lab Chip 2004,4, 98-103.

[54] Borenstein JT, Weinberg EJ, Orrick BK, Sundback C, Kaazempur-Mofrad MR, Vacanti JP. Microfabrication of three-dimensional engineered scaffolds. Tissue Eng 2007, 13, 1837-1844.

[55] Zhang H, Hutmacher DW, Chollet F, Poo AN, Burdet E. Microrobotics and MEMSbased fabrication techniques for scaffold-based tissue engineering. Macromol Biosci 2005, 5, 477-489.

[56] Wendel B, Rietzel D, Kühnlein F, Feulner R, Hülder G, Schmachtenberg E. Additive processing of polymers. Macromol Mater Eng 2008, 293, 799-809.

[57] Melchels FPW, Feijen J, Grijpma DW. A review on stereolithography and its applications in biomedical engineering. Biomaterials 2010, 31, 6121-6130.

[58] Hutmacher DW, Sittinger M, Risbud MV. Scaffold-based tissue engineering: rationale for computer-aided design and solid free-form fabrication systmens. Trends Biotechnol 2004, 22, 354-362.

[59] Savalani MM, Harris RA. Layer manufacturing for in vivo devices. $P$ I Mech Eng $H$ 2006, 220, 505-520.

[60] Park S-H, Yang D-Y, Lee K-S. Two-photon stereolithography for realizing ultraprecise three-dimensional nano/microdevices. Laser Photonics Rev 2009, 3, 1-11.

[61] Mankovich NJ, Samson D, Pratt W, Lew D, Beumer J. Surgical planning using 3dimensional imaging and computer modeling. Otolaryng Clin N Am 1994, 27, 875889.

[62] Melchels FPW, Feijen J, Grijpma DW. A poly(D,L-lactide) resin for the preparation of tissue engineering scaffolds by stereolithography. Biomaterials 2009, 30, 38013809.

[63] Melchels FPW, Bertoldi K, Gabbrielli R, Velders AH, Feijen J, Grijpma DW. Mathematically defined tissue engineering scaffold architectures prepared by stereolithography. Biomaterials 2010, 31, 6909-6916.

[64] Tsang VL, Bhatia SN. Three-dimensional tissue fabrication. Adv Drug Deliv Rev 2004, 56, 1635-1647.

[65] Cooke MN, Fisher JP, Dean Dm, Rimnac C, Mikos AG. Use of stereolithography to manufacture critical-sized 3D biodegradable scaffolds for bone ingrowth. J Biomed Mater Res 2003, 64B, 65-69.

[66] Lee JW, Lan PX, Kim B, Lim G, Cho D-W. 3D scaffold fabrication with PPF/DEF using micro-stereolithography. Microelectron Eng 2007, 84, 1702-1705.

[67] Dhariwala B, Hunt E, Boland T. Rapid prototyping of tissue-engineerign constructs, using photopolymerizable hydrogels and stereolithography. Tissue Eng 2004, 10, 1316-1322.

[68] Mapili G, Lu Y, Chen S, Roy K. Laser-layered microfabrication of spatially patterned functionalized tissue-engineering scaffolds. J Biomed Mater Res B 2005, 75B, 414424.

[69] Jansen J, Melchels FPW, Grijpma DW, Feijen J. Fumaric acid monoethyl esterfunctionalized poly(D,L-lactide)/N-vinyl-2-pyrrolidone resins for the preparation of tissue engineering scaffolds by stereolithography. Biomacromolecules 2009, 10, 214220. 
[70] Matsuda T, Mizutani M. Liquid acrylate-endcapped biodegradable poly( $\varepsilon$ caprolactone-co-trimethylene carbonate). II. Computer-aided stereolithographic microarchitectural surface photoconstructs. J Biomed Mater Res 2002, 62, 395-403.

[71] Kwon KI, Matsuda T. Photo-polymerized microarchitectural constructs prepared by microstereolithography ( $\mu \mathrm{SL})$ using liquid acrylate-end-capped trimethylene carbonate-based prepolymers. Biomaterials 2005, 26, 1675-1684.

[72] Lee S-J, Kang H-W, Park JK, Rhie J-W, Hahn SK, Cho D-W. Application of microstereolithography in the development of three-dimenstional cartilage regeneration scaffolds. Biomed Microdevices 2008, 10, 233-241.

[73] Mapili G, Lu Y, Chen S, Roy K. Laser-layered microfabrication of spatially patterned functionalized tissue-engineering scaffolds. J Biomed Mater Res Part B: Appl Biomater 2005, 75B, 414-424.

[74] Dhariwala B, Hunt E, Boland T. Rapid prototyping of tissue-engineering constructs, using photopolymerizable hydrogels and stereolithography. Tissue Eng 2004, 10, 1316-1322.

[75] Langer R, Vacanti JP. Tissue engineering. Science 1993, 260. 920-926.

[76] Karp JM, Dalton PD, Shoichet MS. Scaffolds for tissue engineering. MRS Bull 2003, 28, 301-306.

[77] Yang SF. Leong KF, Du ZH, Chua CK. The design of scaffolds for use in tissue engineering. Tissue Eng 2001, 7, 679-689.

[78] Griffith LG, Swartz MA. Capturing complex 3D tissue physiology in vitro. Nat Rev Mol Cell Biol 2006, 7, 211-224.

[79] Tan W, Desai TA. Microscale multilayer cocultures for biomimetic blood vessels. $J$ Biomed Mater Res A 2005, 72A, 146-160.

[80] Tan W, Desai TA. Layer-by-layer microfluidics for biomimetic three-dimensional structures. Biomaterials 2004, 25, 1355-1364.

[81] Freed LE, Engelmayr GC, Borenstein JT, Moutos FT, Guilak F. Advanced material strategies for tissue engineering scaffolds. Adv Mater 2009, 21, 3410-3418.

[82] Griffith CK, Miller C, Sainson RCA, Calvert JW, Jeon NL, Hughes CCW, George SC. Diffusion limits of an in vitro thick prevascularized tissue. Tissue Eng 2005, 11, 257-266.

[83] Carothers WH, van Natta FJ. Studies on polymerization and ring formation. III Glycol esters of carbonic acid. J Am Chem Soc 1930, 52, 314-326.

[84] Zhu KJ, Hendren RW, Jensen K, Pitt CG. Synthesis, properties, and biodegradation of poly(1,3-trimethylene carbonate). Macromolecules 1991, 24, 1736-1740.

[85] Zhang Z, Kuijer R, Bulstra SK, Grijpma DW, Feijen J. The in vivo and in vitro degradation behavior of poly(trimethylene carbonate). Biomaterials 2006, 27, 17411748.

[86] Pêgo AP, Vleggeert-Lankamp CLAM, Deenen M, Lakke EAJF, Grijpma DW, Poot AA, Marani E, Feijen J. Adhesion and growth of human schwann cells on trimethylene carbonate (co)polymers. J Biomed Mater Res A 2003, 67A, 876-885.

[87] Pêgo AP, Van Luyn MJA, Brouwer LA, Van Wachem PB, Poot AA, Grijpma DW, Feijen J. In vivo behavior of poly(1,3-trimethylene carbonate) and copolymers of 1,3trimethylene carbonate with D,L-lactide or epsilon-caprolactone: degradation and tissue response, J Biomed Mater Res A. 2003, 67A, 1044-1054.

[88] Fabre T, Schappacher M, Bareille R, Dupuy B, Soum A, Bertrand-Barat J, Baquey C. Study of a (trimethylenecarbonate-co-epsilon-caprolactone) polymer - part 2: In vitro cytocompatibility analysis and in vivo ed 1 cell response of a new nerve guide. Biomaterials 2001, 22, 2951-2958. 
[89] Katz AR, Mukherjee DP, Kaganov AL, Gordon S. A new synthetic monofilament absorbable suture made from polytrimethylene carbonate. Surg Gynecol Obstet 1985, 161, 213-222.

[90] Engelberg I, Kohn J. Physicomechanical properties of degradable polymers used in medical applications - a comparative-study. Biomaterials 1991, 12, 292-304.

[91] Pego AP, Grijpma DW, Feijen J. Enhanced mechanical properties of 1,3-trimethylene carbonate polymers and networks. Polymer 2003, 44, 6495-6504.

[92] Pêgo AP. Poot AA, Grijpma DW, Feijen J. Copolymers of trimethylene carbonate and epsiolon-caprolactone for porous nerve guides: Synthesis and properties. J Biomater Sci-Polym E 2001, 12, 35-53.

[93] Pêgo AP, Siebum B, Van Luyn MJA, Gallego XJ, Van Seijen Y, Poot AA, Grijpma DW, FeijenJ. Preparation of degradable porous structures based on 1,3-trimethylene carbonate and D,L-lactide (co)polymers for heart tissue engineering. Tissue Eng 2003, 9, 981-994.

[94] Timbart L, Tse MY, Pang SC, Babasola O, Amsden BG. Low viscosity poly(trimethylene carbonate) for localized drug delivery: pheological properties and in vivo degradation. Macromol Biosci 2009, 9, 786-794.

[95] Chapanian R, Tse MY, Pang SC, Amsden BG. The role of oxidation and enzymatic hydrolysis on the in vivo degradation of trimethylene carbonate based photocrosslinkable elastomers. Biomaterials 2009, 30, 295-306.

[96] Matsuda T, Mizutani M. Molecular design of photocurable liquid biodegradable copolymers. 2. Synthesis of coumarin-derivatized oligo(Methacrylate)s and photocuring. Macromolecules 2000, 33, 791-794.

[97] Matsuda T, Mizutani M, Arnold SC. Molecular design of photocurable liquid biodegradable copolymers. 1. Synthesis and photocuring characteristics. Macromolecules 2000, 33, 795-800.

[98] Bat E, Plantinga JA, Harmsen MC, Van Luyn MJA, Zhang Z, Grijpma DW, Feijen J. Trimethylene carbonate and $\varepsilon$-caprolactone based (co)polymer networks: mechanical properties and enzymatic degradation. Biomacromolecules 2008, 9, 3208-3215.

[99] Pego AP, Van Luyn MJA, Brouwer LA, van Wachem PB, Poot AA, Grijpma DW, Feijen J. In vivo behavior of poly(1,3-trimethylene carbonate) and copolymers of 1,3trimethylene carbonate with D,L-lactide or epsilon-caprolactone: degradation and tissue response. J Biomed Mater Res A 2003, 67A, 1044-1054.

[100] Mizutani M, Matsuda T. Liquid photocurable biodegradable copolymers: In vivo degradation of photocured poly( $\varepsilon$-caprolactone-co-trimethylene carbonate). J Biomed Mater Res 2002, 61, 53-60.

[101]Bat E, Plantinga JA, Harmsen MC, Van Luyn MJA, Feijen J, Grijpma DW. In vivo behavior of trimethylene carbonate and $\varepsilon$-caprolactone-based (co)polymer networks: degradation and tissue response. J Biomed Mater Res A 2010, 95A, 940-949.

[102] Papenburg BJ, Schüller-Ravoo S, Bolhuis-Versteeg LAM, Hartsuiker L, Grijpma DW, Feijen J, Wessling M, Stamatialis D. Designing porosity and topography of poly(1,3-trimethylene carbonate) scaffolds. Acta Biomater 2009, 5, 3281-3294.

[103] Song Y, Kamphuis MMJ, Zhang Z, Sterk LMT, Vermes I, Poot AA, Feijen J, Grijpma DW. Flexible and elastic porous poly(trimethylene carbonate) structures for use in vascular tissue engineering. Acta Biomater 2010, 6, 1269-1277.

[104] Song Y, Wennink JWH, Kamphuis MMJ, Vermes I, Poot AA, Feijen J, Grijpma DW. Effective seeding of smooth muscle cells into tubular poly(trimethylene carbonate) scaffolds for vascular tissue engineering. J Biomed Mater Res A, 2010, 95A, 440-446. 


\title{
Chapter 3
}

\section{Flexible, Elastic and Tear-Resistant Networks Prepared by Photo-Crosslinking Poly(Trimethylene Carbonate) Macromers}

\begin{abstract}
Poly(trimethylene carbonate) (PTMC) macromers with molecular weights between 1000 and $41000 \mathrm{~g} / \mathrm{mol}$ were prepared by ring opening polymerization and subsequent functionalization with methacrylate end groups. Flexible networks were obtained by radical photo-crosslinking reactions of these macromers. With increasing molecular weight of the macromer, the obtained networks showed increasing swelling ratios in chloroform and decreasing glass transition temperatures, reaching a constant value of approximately $-18{ }^{\circ} \mathrm{C}$. This value is close to that of linear high molecular weight PTMC.

For all prepared networks the creep resistance was high. It was found that the molecular weight of the macromer strongly influenced the tensile properties of the networks obtained after photo-crosslinking. At room temperature, the E-modulus decreased from $314 \mathrm{MPa}$ for the network prepared from the macromer with the lowest molecular weight to a value of $5 \mathrm{MPa}$ for the network prepared from the macromer with the highest molecular weight. The elongation at break of the networks continuously increased with molecular weight of the macromer to reach a very high value of $1200 \%$. The maximum values for the tensile strengths of the networks were found to first decrease with increasing macromer molecular weight. At molecular weights higher than approximately $10000 \mathrm{~g} / \mathrm{mol}$ the networks showed rubber-like behavior and the maximum tensile strengths increased with macromer molecular weight. Determinations of the toughness (the area under the stress-strain curves, W) determined in tensile testing experiments, in tear propagation experiments, and in suture retention strength measurements showed that PTMC networks prepared from the higher molecular weight macromers (molecular weights higher than $10000 \mathrm{~g} / \mathrm{mol}$ ) were tenacious materials.

The mechanical properties of these networks compare favorably with those of linear high molecular weight PTMC and with those of well-known elastomeric materials like silicone
\end{abstract}


rubber (poly(dimethylsiloxane), PDMS) and latex natural rubber. The mechanical properties of the networks also compare favorably with those of native blood vessels, which may be of importance for using these materials for the tissue-engineering of small diameter blood vessels.

\section{Introduction}

Much attention has recently been paid to developing biodegradable elastic materials for the preparation of soft tissue implants and soft tissue engineering scaffolds for use in cardiovascular applications. As suturing is the most common technique to connect grafts to soft tissue, the materials are ideally suturable and resistant to tearing. ${ }^{[1-3]}$ When suturing, a defect is created that in a two-step tearing process can lead to catastrophic failure of the implant. ${ }^{[4]}$ Nevertheless, research on developing suturable, tough and tear resistant biodegradable elastomeric implant materials is very limited.

We have prepared flexible and elastic creep resistant networks that are biocompatible and biodegradable from high molecular weight poly(trimethylene carbonate) (PTMC). The polymer crosslinks when exposed to gamma-radiation, and form-stable networks with excellent mechanical properties, elasticity, and resistance to creep can be obtained in this manner. ${ }^{[5-6]}$ Also, elastic porous PTMC structures for use in vascular tissue engineering could be prepared in this way. ${ }^{[7]}$ However, the crosslinking procedure is quite involved as gamma-irradiation of the polymers needs to be done externally in a specialized facility. Photo-crosslinking of precursor macromers would be a much more practical and versatile approach to prepare tear- and creep resistant PTMC networks.

In early work by Storey and co-workers, three-armed oligomeric polyesters based on D,L-lactide and trimethylene carbonate with molecular weights between approximately 2300 and $2600 \mathrm{~g} / \mathrm{mol}$ were end-capped with methacrylate groups and cured by thermal radical polymerization to yield amorphous network structures. ${ }^{[8]}$ The networks with 80 and $100 \%$ of trimethylene carbonate were flexible with low values of the E-modulus. Matsuda et al. prepared biodegradable networks by photo-crosslinking liquid acrylate-end-capped poly( $\varepsilon$-caprolactone-co-trimethylene carbonate) macromers with molecular weights of 2600 and $3500 \mathrm{~g} / \mathrm{mol}$ using stereolithography. ${ }^{[9]}$ The authors did not discuss the mechanical properties of their materials, but cracks could be discerned in the built structures and in some cases the structures were found to have been broken upon subcutaneous implantation 
in rats. Most likely, the rubber-like materials of the previous examples would also be too brittle to be suturable. The molecular weights of the macromers from which the amorphous networks were prepared were quite low, resulting in high crosslinking densities and restricted mobility of the network chains leading to brittle behaviour. ${ }^{[10-11,13]}$

Tough and tear resistant rubber-like materials are obtained when propagation of a microfracture or tear is hindered. Examples of elastomeric materials in which the propagation of a tear is hindered are natural and silicone rubber networks to which filler particles have been added, elastomeric networks prepared from block copolymers comprising crystallisable segments such as polyurethanes, elastomeric networks prepared from chains with bimodal chain length distributions, and networks that show strain induced crystallization. ${ }^{[12-13]}$ Natural rubber displays this self-reinforcing behavior and has excellent toughness, tear resistance, and tensile properties. ${ }^{[14]}$

In general unmodified non-phase separated amorphous polymer networks that display rubber-like elasticity are weak and fracture catastrophically at moderate elongations as a result of their poor tear resistance. ${ }^{[12]}$ Hou et al. have shown that, when photo-crosslinking PTMC macromers of different molecular weights, the tensile strength and elongation at break of the resulting networks significantly increased with increasing molecular weight. ${ }^{[15]}$ Preliminary experiments in which the tear strength of PTMC networks prepared from PTMC macromers with different molecular weights was determined indicated that in this manner much tougher and more tear resistant PTMC networks could be prepared.

In this paper we describe the preparation of tough, tear-resistant networks by photo-crosslinking methacrylate functionalized PTMC macromers. The influence of the molecular weight of the macromer on the mechanical properties of the obtained networks, especially regarding their elasticity and their toughness, suturability and resistance to tearing, is evaluated. To assess the potential of photo-crosslinked PTMC networks as blood vessel grafts or as blood vessel tissue engineering scaffolds, the mechanical properties of native blood vessels were evaluated as well. 


\section{Materials and Methods}

\section{Materials}

Polymer grade 1,3-trimethylene carbonate (1,3-dioxan-2-one, TMC) was obtained from Boehringer Ingelheim (Germany). Glycerol (99.5+\% spectrophotometric grade) was purchased from Aldrich (USA) and dehydrated by briefly heating at $180{ }^{\circ} \mathrm{C}$. Stannous octoate (stannous 2-ethylhexanoate, $\mathrm{SnOct}_{2}$ ) was used as received from Sigma (USA). Triethyl amine (used as received) and methacryloyl chloride (distilled prior to use) were obtained from Fluka (Germany). Methacrylic anhydride (94\%) and hydroquinone were purchased from Aldrich (Germany), and ( \pm )- $\alpha$-Tocopherol (vitamin E) from Fluka (Switzerland). Irgacure 2959 (1-[4-(2-hydroxyethoxy)-phenyl]-2-hydroxy-2-methyl-1propane-1-one) was provided by Ciba Specialty Chemicals (Switzerland).

Dichloromethane of analytical grade was purchased from Biosolve (The Netherlands), dried over calcium hydride and freshly distilled. Technical grade chloroform and ethanol were obtained from Chempopack (Belgium). Serva silicone solution was purchased from SERVA Electrophoresis GmbH (Germany). Propylene carbonate was purchased from Merck (Germany).

Linear, high molecular weight (HMW) PTMC $\left(\overline{\mathrm{M}}_{n}=243000 \mathrm{~g} / \mathrm{mol}, \mathrm{T}_{\mathrm{g}}=-15.7^{\circ} \mathrm{C}\right)$ was synthesized by ring opening polymerization. Films with a thickness of $500 \mu \mathrm{m}$ were prepared by compression moulding at $140{ }^{\circ} \mathrm{C}$ and used as a reference material. Also, silicone elastomer (Sylgard ${ }^{\circledR} 184$, Dow Corning, USA) and latex natural rubber (Kars, The Netherlands) films were used as reference materials. Both were cast at a thickness of approximately $400 \mu \mathrm{m}$ and cured at $80{ }^{\circ} \mathrm{C}$ for $24 \mathrm{~h}$.

\section{Synthesis and Characterization of PTMC Macromers}

Star-shaped hydroxyl-terminated PTMC oligomers were prepared by ring opening polymerization (oligomerization) of TMC using glycerol as initiator and $\mathrm{SnOct}_{2}(0.07 \mathrm{wt} \%$ ) as catalyst. The TMC monomer and glycerol initiator were charged into a freshly silanized and dried three-necked flask and polymerized in an argon atomosphere at $130{ }^{\circ} \mathrm{C}$ for 2 days. The molecular weights of the three-armed oligomers could be controlled by adjusting the TMC to glycerol ratio. 
Subsequently, the hydroxyl-terminated oligomers were functionalized by reaction with methacryloyl chloride or with methacrylic anhydride. The oligomers with the higher molecular weights (approximately 18000 to $41000 \mathrm{~g} / \mathrm{mol}$, determined by NMR) were functionalized with methacryloyl chloride in dichloromethane solutions at room temperature. To PTMC oligomer solutions (1.3 to $4.0 \mathrm{mmol}$ in 150 to $200 \mathrm{ml}$ dichloromethane), an excess of triethyl amine $(0.25 \mathrm{mmol}$ excess per hydroxyl group, also dissolved in $10 \mathrm{ml}$ dichloromethane) was added. The solutions were then cooled with an ice bath, and methacryloyl chloride (an excess of 0.25 mmol per hydroxyl group in $30 \mathrm{ml}$ dichloromethane) was slowly added while vigorously stirring. The contents were let to heat up to room temperature, and the reaction was continued for at least 2 days. Purification of most of these macromers could be readily achieved by precipitation into cold ethanol and drying under vacuum.

As the macromers of the lower molecular weights (1000 to $10300 \mathrm{~g} / \mathrm{mol}$ ) could not be easily purified by precipitation, the functionalization of the corresponding oligomers was conducted using methacrylic anhyride, followed by distillation of the excess methacrylic anhydride and formed methacrylic acid. The PTMC oligomers were dissolved in methacrylic anhydride (an excess of $25 \mathrm{~mol} \%$ per functional hydroxyl group was used) and reacted at $120{ }^{\circ} \mathrm{C}$ for 1 to 5 days. To avoid premature crosslinking, $0.06 \mathrm{wt} \%$ hydroquinone and $0.06 \mathrm{wt} \%( \pm)$ - $\alpha$-tocopherol (both relative to the oligomer) were used. The macromers were purified by removal of the formed methacrylic acid and the excess methacrylic anhydride by vacuum distillation.

After vacuum distillation, the macromer with molecular weight $10300 \mathrm{~g} / \mathrm{mol}$ still contained methacrylic anhydride, and was additionally purified by precipitation into cold ethanol and drying under vacuum.

Monomer conversions in the oligomerization reaction, molecular weights and degrees of functionalization of the macromers were determined by ${ }^{1} \mathrm{H}-\mathrm{NMR}$ spectroscopy (Varian Innova $300 \mathrm{MHz}$, USA), using $\mathrm{CDCl}_{3}$ (Merck, Germany) as solvent.

Thermal properties of macromers were determined by differential scanning calorimetry (DSC) employing a Perkin Elmer Pyris 1 (USA). Samples of 5-12 mg were heated from $-100{ }^{\circ} \mathrm{C}$ to $100{ }^{\circ} \mathrm{C}$ at a heating rate of $10{ }^{\circ} \mathrm{C} / \mathrm{min}$ and quenched rapidly $\left(300{ }^{\circ} \mathrm{C} / \mathrm{min}\right)$ to $-100{ }^{\circ} \mathrm{C}$. After $5 \mathrm{~min}$, a second scan was recorded. Melting temperatures $\left(\mathrm{T}_{\mathrm{m}}\right)$ were taken as peak melting temperatures in the first scan, while glass transition temperatures $\left(\mathrm{T}_{\mathrm{g}}\right)$ were determined using the second scan data. To give a measure of the width of the glass 
transition, onset values ( $\mathrm{T}_{\mathrm{g}}$ (onset)) and midpoint values of the heat capacity change $\left(\mathrm{T}_{\mathrm{g}}(\right.$ midpoint $\left.)\right)$ are reported. Temperature calibration was performed using cyclohexane, indium and lead as calibration standards.

\section{Preparation of Networks by Photo-Crosslinking of PTMC Macromers}

PTMC networks were prepared by photo-crosslinking in bulk. First, chloroform solutions containing 15 to $45 \mathrm{wt} \%$ PTMC macromer and $5 \mathrm{wt} \%$ (relative to the macromer) Irgacure 2959 photoinitiator were poured into glass molds, then the solvent was allowed to evaporate. To prevent premature crosslinking, this was done in the dark. The liquid macromers $\left(\overline{\mathrm{M}}_{n}=1000\right.$ and $\left.1800 \mathrm{~g} / \mathrm{mol}\right)$ were then photo-crosslinked at room temperature under nitrogen in a crosslinking cabinet (Ultralum, USA) for $5 \mathrm{~min}$ at $365 \mathrm{~nm}$. PTMC macromers with molecular weights of 3300 to $41000 \mathrm{~g} / \mathrm{mol}$ were very viscous or semi-crystalline materials, therefore these were crosslinked at $70{ }^{\circ} \mathrm{C}$ under nitrogen in a crosslinking cabinet (Ultralum, USA) for $5 \mathrm{~min}$ at $365 \mathrm{~nm}$.

\section{Characterization of Photo-Crosslinked PTMC Networks}

The obtained transparent films were extracted with a mixture of propylene carbonate (70 vol\%) and chloroform (30 vol\%), the solvent was refreshed twice. The extracted films were then washed with ethanol and dried under vacuum until a constant weight was reached. The dry extracted films were $0.5 \pm 0.2 \mathrm{~mm}$ thick.

The gel content of the photo-crosslinked networks was determined in triplicate using equation 1:

$$
\text { gel content }(\%)=\frac{m_{d r y}}{m_{\text {initial }}} \times 100
$$

where $m_{d r y}$ is the mass of the insoluble part of the network after drying and $m_{\text {initial }}$ is the mass of the crosslinked network before extraction.

The volume degree of swelling $(q)$ was assessed by swelling the extracted networks in chloroform, the values were determined according to equation 2 :

$$
q=1+\left(\frac{m_{s w o l l e n}-m_{d r y}}{m_{d r y}}\right) \times \frac{\rho_{p}}{\rho_{s}}
$$


where $m_{\text {swollen }}$ is the weight of the swollen network, and $\rho_{s}$ and $\rho_{p}$ are the densities of chloroform $\left(1.48 \mathrm{~g} / \mathrm{cm}^{3}\right)$ and PTMC $\left(1.31 \mathrm{~g} / \mathrm{cm}^{3}\right)$, respectively.

\section{Thermal and Mechanical Properties of Photo-Crosslinked PTMC Networks}

After extraction and drying, the thermal properties of the networks were determined by DSC in the manner described above.

The mechanical properties of extracted photo-crosslinked PTMC networks were determined using a Zwick Z020 tensile tester (Germany), equipped with a $500 \mathrm{~N}$ load cell at room temperature. The elongation of the specimens was derived from the grip-to-grip separation, which initially was $50 \mathrm{~mm}$. Unless mentioned otherwise, experiments were conducted in triplicate. Tensile properties (Young's modulus, maximum tensile strength and elongation at break) of the PTMC networks were determined according to ASTM D882-91 at a crosshead speed of $50 \mathrm{~mm} / \mathrm{min}$ using specimens measuring $100 \times 5 \mathrm{~mm}^{2}$. The yield stress and the elongation at yield were determined from the intersection of tangents to the stressstrain curves. The toughness (W, expressed in $\mathrm{N} / \mathrm{mm}^{2}$ ) of the specimens in tensile testing was determined as the areas under the stress-strain curves.

Cyclic creep experiments were performed at room temperature. In cyclic tensile tests, the specimens were elongated 20 times to $50 \%$ strain at a rate of $50 \mathrm{~mm} / \mathrm{min}$. After $2 \mathrm{~h}$ relaxation, the permanent deformation (permanent set) was determined from the stressstrain curve of the $21^{\text {st }}$ tensile cycle. Unless otherwise mentioned, the experiments were conducted in twofold.

The average and maximum tear propagation strength (TPS) of the PTMC networks were determined according to ASTM 1938 at a crosshead speed of $250 \mathrm{~mm} / \mathrm{min}$. The trouser model specimens measured $75 \times 25 \mathrm{~mm}^{2}$, and had a $50 \mathrm{~mm}$ long cut. The values for the tear propagation strengths are normalized to the specimen thickness and are given in $\mathrm{N} / \mathrm{mm}$. The maximum suture retention strengths (SRS) were determined according to ANSI/AAMI VP20:1994, using approximately $0.5 \mathrm{~mm}$ thick specimens measuring $45 \times 12.5 \mathrm{~mm}^{2}$. Using a syringe needle $(0.50 \times 16 \mathrm{~mm} / 25 \mathrm{Gx} 5 / 8$, Sterican, Germany) a hole was punctured at a distance of $2 \mathrm{~mm}$ from the top edge of the specimen. Through this hole, a stainless steel wire (0.28 mm diameter, Metalwire, The Netherlands) was inserted. After clamping both ends of the wire in the upper grip of the tensile testing machine and the PTMC network specimen in the lower grip, the force required to tear the specimen was determined at a 
crosshead speed of $150 \mathrm{~mm} / \mathrm{min}$. The value for the maximum SRS was normalized to the specimen thickness and given in $\mathrm{N} / \mathrm{min}$.

The toughness of the specimens (normalized to their thickness) in tear propagation strength measurements ( $\mathrm{W}_{\text {TPS }}$ in $\mathrm{N}$ ) and in suture retention strength measurements ( $\mathrm{W}_{\mathrm{SRS}}$ in $\mathrm{N}$ ) were determined as the areas under the corresponding stress-strain curves.

\section{Mechanical Properties of Natural Blood Vessels}

Ovine carotid arteries, and porcine carotid arteries and aortas were obtained from a local slaughterhouse. The blood vessels were harvested immediately after slaughtering, and stored in phosphate buffered solution at $4{ }^{\circ} \mathrm{C}$. The tensile properties of these natural tissues were determined within $72 \mathrm{~h}$ of harvesting as described above. Values of the elasticity modulus were determined at low elongations (Young's modulus) and at high elongations (100-110\% strain). To determine the tensile strength, suture retention strength and tear strength of porcine aortas, specimens were punched out in the longitudinal direction of the aorta. For the smaller carotid arteries, the harvested tubular structures were tested in the longitudinal direction without further sample preparation. 


\section{Results and Discussion}

\section{Synthesis and Characterization of PTMC Macromers}<smiles>O=C1OCCCO1</smiles>

glycerol

TMC

$$
\downarrow \begin{aligned}
& 130^{\circ} \mathrm{C} \\
& 2 \text { days }
\end{aligned}
$$<smiles>[R]OCC(COC(C)C)OCCCOC(C)(C)C</smiles>

PTMC oligomer<smiles>C=C(C)C(=O)Cl</smiles>

room temperature 2 days
$+3$<smiles>C=C(C)C(=O)OC(=O)C(=C)C</smiles>

$120^{\circ} \mathrm{C}$

$1-5$ days<smiles>[R]OCC(COC(C)(C)C)OCCCOC(C)=O</smiles>

PTMC macromer

Figure 1. Scheme showing the synthesis of PTMC macromers: three-armed, hydroxylterminated PTMC oligomers were prepared using glycerol as initiator and subsequently functionalized with methacryloyl chloride or methacrylic anhydride. 
Star-shaped, methacrylate-functionalized PTMC oligomers (macromers) were synthesized by a two-step process, which is illustrated in Figure 1. In the first step trimethylene carbonate (TMC) was polymerized to yield three-armed hydroxyl-terminated PTMC oligomers using glycerol as initiator and stannous octoate as catalyst. The molecular weight of the oligomers could be adjusted by varying the TMC to glycerol molar ratio. The monomer conversion, determined by ${ }^{1} \mathrm{H}-\mathrm{NMR}$ spectroscopy of the crude polymerization product, varied between 95 and 99\%. The number average molecular weight $\left(\overline{\mathrm{M}}_{n}\right)$ of the oligomers was calculated from the ${ }^{1} \mathrm{H}-\mathrm{NMR}$ spectra, assuming that each hydroxyl group of the glycerol initiates polymerization of TMC. An example of an oligomer spectrum is shown in Figure 2A. For the calculation, the peak integral of the repeating $\mathrm{CH}_{2}$-groups at $4.2 \mathrm{ppm}$ or $2.05 \mathrm{ppm}$ ( $\mathrm{a}$ and $\mathrm{b}$ in Figure 2A, respectively) were compared with the peak integrals of the last $\mathrm{CH}_{2}$-group of the chain end at $3.7 \mathrm{ppm}$ (Figure $2 \mathrm{~A}, \mathrm{c}$ ).

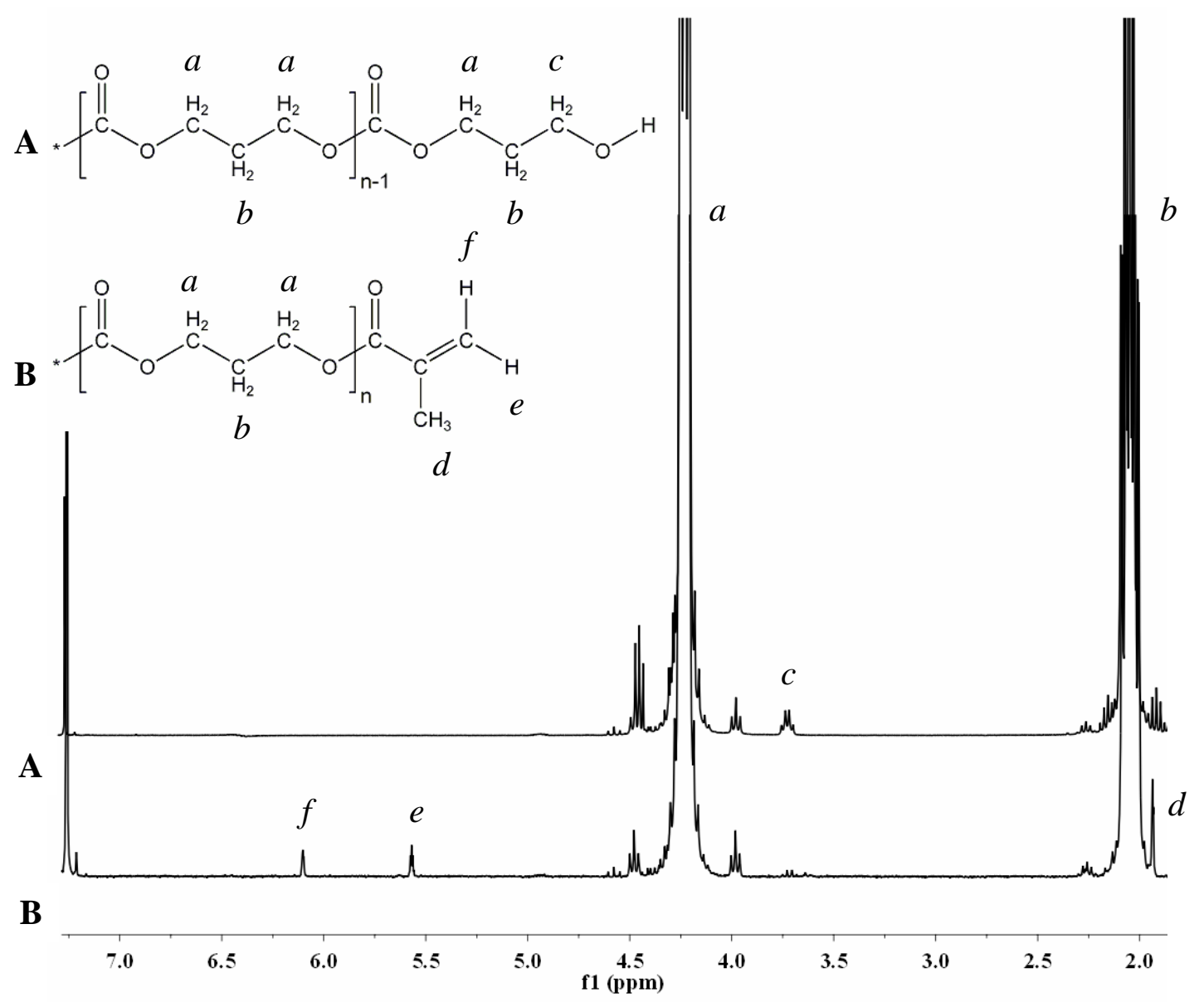

Figure 2. Characteristic ${ }^{1} H$-NMR spectra of three-armed PTMC oligomers (A) and macromers $(B)$ allowing determination of average molecular weights and degrees of functionalization. 
In the second step, the hydroxyl-terminated oligomers were functionalized to give PTMC macromers. Oligomers with the higher molecular weights (approximately 18000 to $41000 \mathrm{~g} / \mathrm{mol}$ ) were functionalized with methacryloyl chloride, while oligomers with lower molecular weights were functionalized using methacrylic anhydride. ${ }^{1} \mathrm{H}-\mathrm{NMR}$ spectroscopy confirmed the functionalization of the oligomers (see Figure $2 \mathrm{~A}$ and $\mathrm{B}$ ). The macromer methacrylate groups could be observed at $6.08 \mathrm{ppm}(\mathrm{CH}), 5.58 \mathrm{ppm}(\mathrm{CH})$ and $1.90 \mathrm{ppm}$ $\left(\mathrm{CH}_{3}\right)$ (respectively f, e and $\mathrm{d}$ in Figure $2 \mathrm{~B}$ ) in the ${ }^{1} \mathrm{H}-\mathrm{NMR}$ spectrum. Comparison of the integral of the methacrylate peak at $6.08 \mathrm{ppm}$ ( $\mathrm{f}$ in Figure 2B) with the integral of the oligo(trimethylene carbonate) peak at $4.2 \mathrm{ppm}$ ( $\mathrm{a}$ in Figure $2 \mathrm{~A}$ ) and taking into account the molecular weight of the PTMC oligomers allowed calculation of the degree of functionalization.

Table 1 shows that the determined degrees of functionalization reached values of up to 94\%. However, as the peak corresponding to the last $-\mathrm{CH}_{2}$ - group of the oligomer chain ends (c in Figure 2A) cannot be discerned upon functionalization, it can be assumed that the functionalizations were essentially complete.

Table 1. Properties of three-armed PTMC macromers containing methacrylate end groups.

\begin{tabular}{|c|c|c|c|c|c|}
\hline $\begin{array}{l}\overline{\mathrm{M}}_{n} \text { of } \\
\text { macromer }\end{array}$ & $\mathbf{D F}^{\mathrm{a}}$ & $\mathbf{T}_{\mathbf{m}}$ & $\underset{\text { (midpoint) }}{\mathbf{T}_{\mathrm{g}}}$ & $\underset{\text { (onset) }}{\mathbf{T}_{\mathrm{g}}}$ & appearance at \\
\hline$(\mathrm{g} / \mathrm{mol})$ & $(\%)$ & $\left({ }^{\circ} \mathbf{C}\right)$ & $\left({ }^{\circ} \mathbf{C}\right)$ & $\left({ }^{\circ} \mathbf{C}\right)$ & \\
\hline 1000 & 94 & - & -54.2 & -56.6 & liquid \\
\hline 1800 & 94 & - & -41.1 & -43.9 & viscous liquid \\
\hline 3300 & n.d. & - & -33.4 & -36.1 & very viscous liquid \\
\hline 10300 & 87 & 32.9 & -29.6 & -32.5 & sticky solid \\
\hline 18900 & 79 & 40.3 & -19.1 & -21.4 & solid \\
\hline 20000 & 94 & 40.7 & -18.4 & -19.7 & solid \\
\hline 24600 & 71 & 39.5 & -16.9 & -19.7 & solid \\
\hline 41000 & 87 & - & -17.5 & -19.3 & solid \\
\hline
\end{tabular}

${ }^{\text {a }}$ Degree of functionalization (DF) determined by ${ }^{1} \mathrm{H}-\mathrm{NMR}$. Note that NMR does not show the presence of terminal hydroxyl groups after functionalization, indicating essentially complete functionalization, see text. n.d.: not determined. 
Characteristic properties of the prepared PTMC macromers are summarized in Table 1. At room temperature the PTMC macromer $1000 \mathrm{~g} / \mathrm{mol}$ is a colourless liquid, whereas with increasing molecular weight the macromers become more viscous. The macromers with the highest molecular weights are solids. All PTMC macromers showed midpoint glass transition temperatures below room temperature, increasing from $-54{ }^{\circ} \mathrm{C}$ to approximately $-17{ }^{\circ} \mathrm{C}$ with increasing molecular weight. Onset values of the glass transition temperatures were slightly lower, indicating a narrow glass transition temperature range. While the macromers with molecular weights of $3300 \mathrm{~g} / \mathrm{mol}$ and lower did not crystallize, the higher molecular weight macromers showed melting peaks between $32{ }^{\circ} \mathrm{C}$ and $41{ }^{\circ} \mathrm{C}$. The macromer with the highest molecular weight of $40000 \mathrm{~g} / \mathrm{mol}$ did not crystallize either; which is in accordance with earlier observations related to linear non-functionalized PTMC. Linear PTMC with molecular weights up to $25000 \mathrm{~g} / \mathrm{mol}$ have been found to be semi-crystalline, with a melting temperature around $36^{\circ} \mathrm{C}^{[16-18]}$ Zhu et al. reported that the amorphous nature of PTMC with higher molecular weights is due to slow crystallization kinetics. ${ }^{[18]}$ Indeed in tensile testing very high molecular weight polymers showed strain induced crystallization. ${ }^{[15]}$

\section{Preparation of Networks by Photo-Crosslinking of PTMC Macromers}

Networks were formed by photo-crosslinking the PTMC macromers using Irgacure 2959 as photoinitiator and long-wavelength UV irradiation $(365 \mathrm{~nm})$ at $70{ }^{\circ} \mathrm{C}$. The lower molecular weight macromers ( $\overline{\mathrm{M}}_{n}=1000$ and $1800 \mathrm{~g} / \mathrm{mol}$ ) were crosslinked at room temperature. After crosslinking, the networks were extracted, dried and characterized. An overview of the network properties is given in Table 2. Under the applied crosslinking conditions PTMC networks with gel contents between 74 and 99\% were prepared. The degree of swelling of the extracted PTMC networks in chloroform increased with increasing macromer molecular weight. As the crosslink density decreases, the network will be able to swell more. It should be noted that PTMC networks prepared from low molecular weight macromers $\left(\overline{\mathrm{M}}_{n}=1000\right.$ and $\left.1800 \mathrm{~g} / \mathrm{mol}\right)$ were brittle and had fragmented during the extraction process. 
Table 2. Properties of PTMC networks prepared by photo-crosslinking three-armed PTMC macromers.

\begin{tabular}{|c|c|c|c|c|}
\hline $\begin{array}{l}\overline{\mathrm{M}}_{n} \text { of } \\
\text { macromer }\end{array}$ & gel content & volume degree & $\begin{array}{c}\mathbf{T}_{\mathbf{g}} \\
\text { (onset) }\end{array}$ & $\underset{\text { (midpoint) }}{\mathbf{T}_{\mathbf{g}}}$ \\
\hline $\mathrm{g} / \mathrm{mol}$ & $\%$ & & ${ }^{\circ} \mathbf{C}$ & ${ }^{\circ} \mathbf{C}$ \\
\hline 1000 & $95.3 \pm 0.7$ & $3.1 \pm 0.1$ & -12.6 & 7.6 \\
\hline 1800 & $96.0 \pm 0.1$ & $3.4 \pm 0.4$ & -12.1 & -2.9 \\
\hline 3300 & $82.7 \pm 0.3$ & $4.7 \pm 0.2$ & -12.4 & -7.5 \\
\hline 10300 & $77.9 \pm 1.3$ & $8.5 \pm 0.6$ & -18.9 & -16.3 \\
\hline 18900 & $80.9 \pm 0.6$ & $9.8 \pm 0.4$ & -19.9 & -18.2 \\
\hline 20000 & $99.5 \pm 1.0$ & $10.2 \pm 0.3$ & -19.5 & -17.3 \\
\hline 24600 & $83.3 \pm 0.3$ & $12.3 \pm 0.4$ & -19.6 & -17.6 \\
\hline 41000 & $74.1 \pm 0.5$ & $16.9 \pm 0.8$ & -17.2 & -15.4 \\
\hline
\end{tabular}

All PTMC networks were transparent and amorphous with (midpoint) glass transition temperatures ranging from $8{ }^{\circ} \mathrm{C}$ for the network prepared from the $1000 \mathrm{~g} / \mathrm{mol}$ macromer to $-15{ }^{\circ} \mathrm{C}$ for the network prepared from the $41000 \mathrm{~g} / \mathrm{mol}$ macromer. The decrease in glass transition temperatures is due to the increasing mobility of longer network chains. The temperature range of the glass transition of the network prepared from the lowest molecular weight macromer is very broad, as can be seen from the values for $T_{g}$ (onset) and $\mathrm{T}_{\mathrm{g}}$ (midpoint). With increasing macromer molecular weight of the PTMC, the range of the glass transitions becomes smaller.

Figure 3 compares the midpoint glass transition temperatures of PTMC macromers and their corresponding PTMC networks. It can be seen that while the $\mathrm{T}_{\mathrm{g}}$ of low molecular weight macromers is very low, the $\mathrm{T}_{\mathrm{g}}$ of the corresponding networks is very high due the decreased chain mobility in densely crosslinked networks. The glass transition temperatures of the macromers increased with increasing molecular weight, while that of the corresponding networks decreased. These glass transition temperatures approach the value of linear high molecular weight PTMC $\left(-15.7^{\circ} \mathrm{C}\right.$ for PTMC with $\left.\overline{\mathrm{M}}_{n}=243000 \mathrm{~g} / \mathrm{mol}\right)$. 


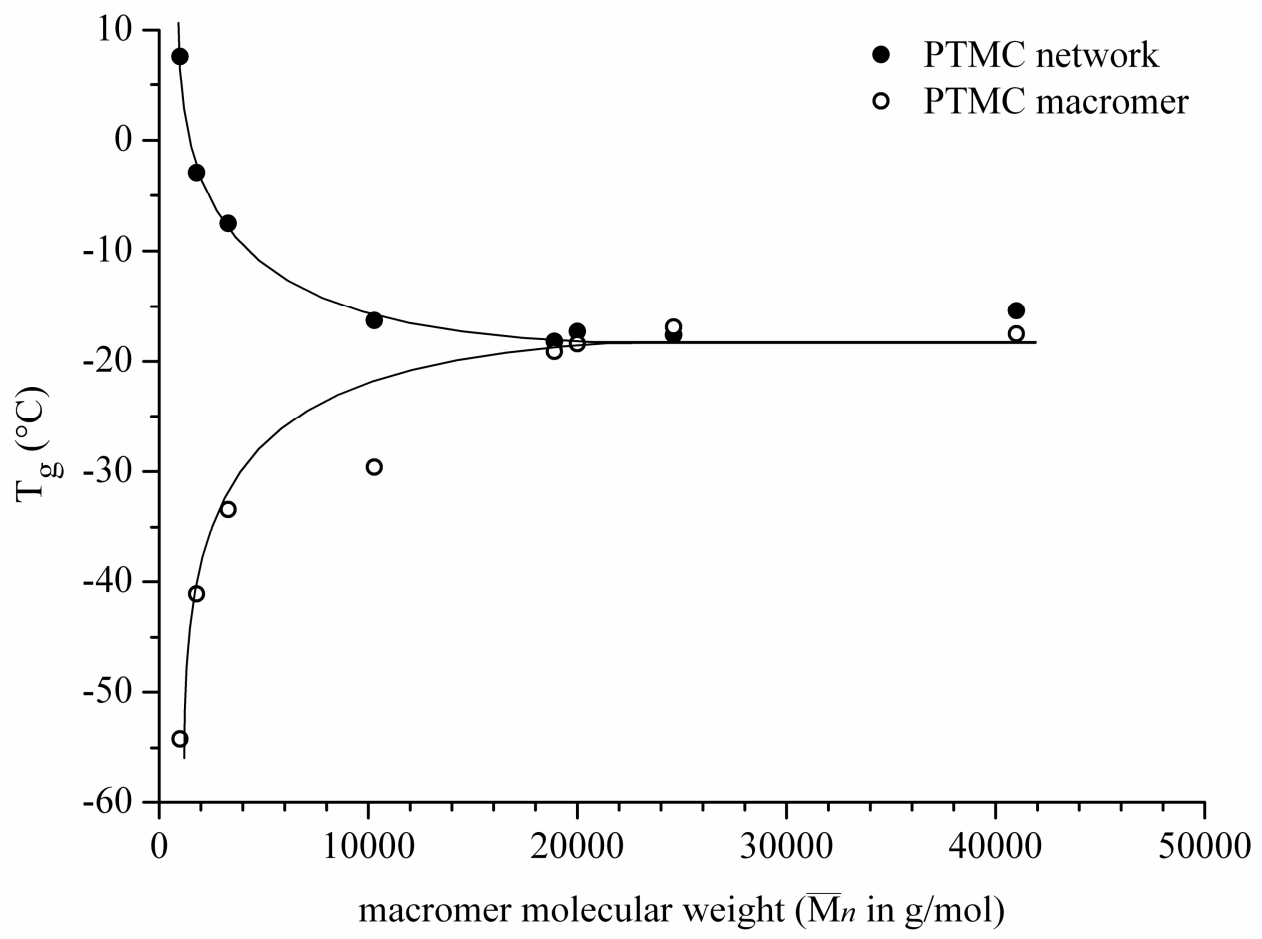

Figure 3. Midpoint glass transition temperatures $\left(T_{g}\right)$ of three-armed PTMC macromers and of corresponding extracted networks as a function of the macromer molecular weight.

\section{Mechanical Properties of PTMC Networks}

To be able to make use of these low $\mathrm{T}_{\mathrm{g}}$ materials, for example in tissue engineering where scaffolds are required in long term cell culturing, form stability is required. By varying the macromer molecular weight, PTMC networks with a wide range of properties could be prepared. Rigid and brittle materials were obtained when low molecular weight macromers were crosslinked, while crosslinking of macromers with relatively high molecular weight resulted in flexible, rubber-like elastomers.

Figure 4 illustrates the effect of the macromer molecular weight on the tensile properties of the resulting networks. The low molecular weight PTMC macromers yielded relatively stiff networks with low elongations at break. With increasing macromer molecular weight, the elastic modulus of the networks decreased and their elongation at break increased. The maximum tensile strength of the networks first decreased and then increased with the macromer molecular weight. Most importantly, networks prepared from macromers with $\overline{\mathrm{M}}_{n}$ values higher than approximately $10000 \mathrm{~g} / \mathrm{mol}$ showed yielding behavior and rubber plateaus, and the areas under the stress-strain curve (which are related to toughness) 
increased significantly. Therefore, these tough, flexible and elastic networks will be very well suited for use as biodegradable soft tissue implants.

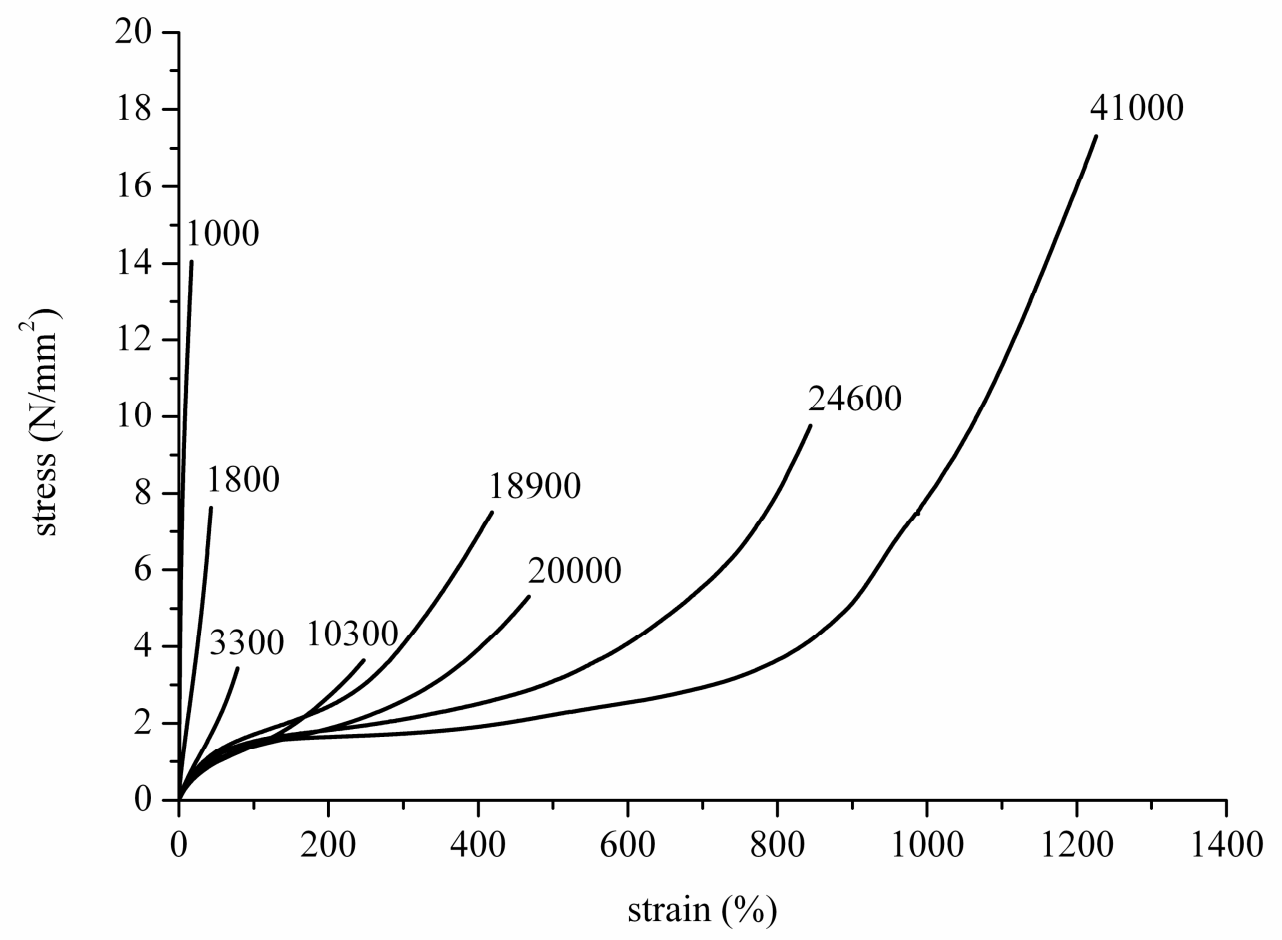

Figure 4. Stress-strain curves for PTMC networks obtained by photo-crosslinking three-armed macromers of different molecular weights $\left(\overline{\mathrm{M}}_{n}\right.$ values of the macromers corresponding to the stress-strain curves are given in the figure).

Table 3 gives an overview of the tensile properties of the photo-crosslinked PTMC networks, of linear high molecular weight PTMC and of other relevant elastomeric networks. 
Table 1. Tensile properties of extracted PTMC networks prepared by photo-crosslinking three-armed PTMC macromers, of linear high molecular weight PTMC and of other relevant elastomeric networks. Triplicate measurements, values are averages \pm standard deviation.

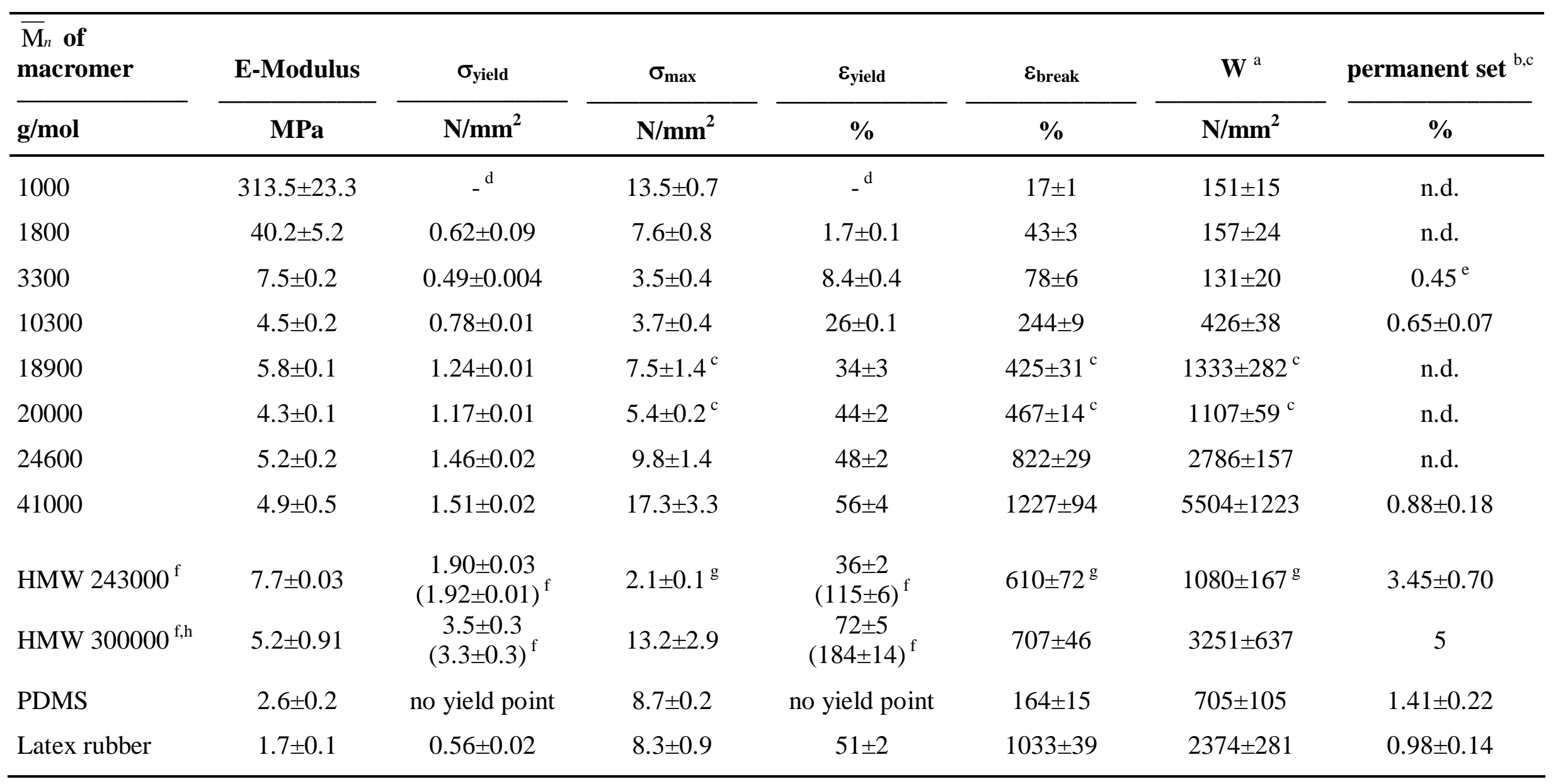

${ }^{\text {a }}$ Normalized area under the stress-strain curve (W, toughness).

${ }^{\mathrm{b}}$ Permanent deformation after 20 cycles in cyclic tensile testing to $50 \%$ elongation.

${ }^{\mathrm{g}}$ Premature failure; specimens broke in grips.

${ }^{\mathrm{c}} \mathrm{n}=2$; results are given as averages \pm standard deviation.

${ }^{\mathrm{h}}$ Data from reference 15.

${ }^{\mathrm{d}}$ Brittle fracture.

n.d. $=$ not determined.

${ }^{\mathrm{e}}$ Specimens failed during cyclic tensile testing to $50 \%$ elongation. Cyclic tensile testing to $25 \%$ elongation, $\mathrm{n}=1$.

${ }^{\mathrm{f}}$ Linear HMW PTMC shows a clear yield point, corresponding values are given in brackets. 
In Figure 5, the values for the maximum tensile strength, elongation at break, tensile yield strength, and elongation at yield of the networks are presented as a function of the molecular weight of the macromers used. While the yield strength, yield strain, and the elongation at break continuously increased with the macromer molecular weight, the maximum tensile strength showed minimum values at molecular weights of 3000 to $10000 \mathrm{~g} / \mathrm{mol}$. This is related to the brittle nature of the densely crosslinked networks in which neither a clear yield point nor rubber-plateau can be discerned. The maximum tensile strength and elongation at break of the networks then increased again with increasing macromer molecular weight.
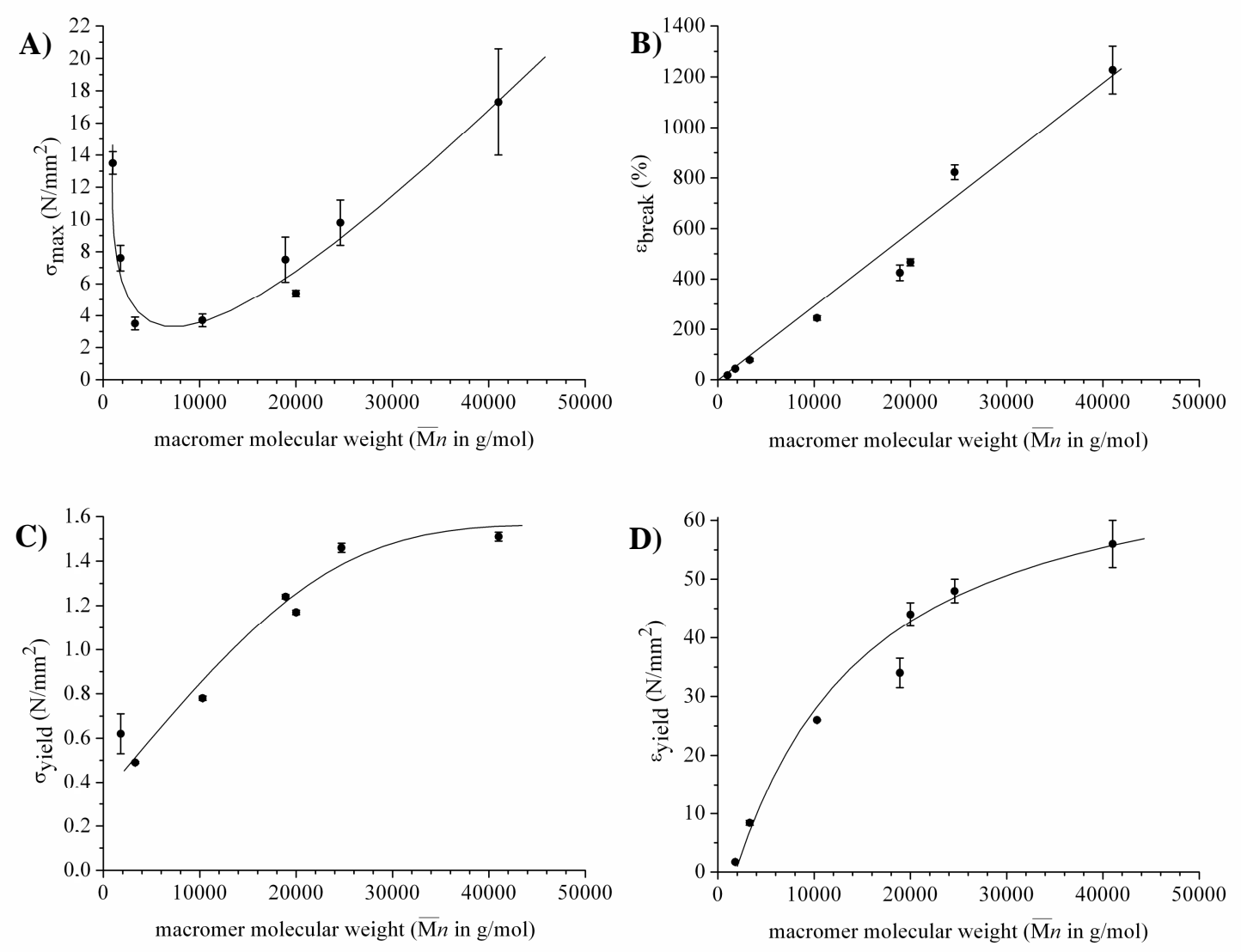

Figure 5. Illustration of the tensile properties of photo-crosslinked PTMC networks as a function of the molecular weight of the three-armed macromers used. Maximum tensile strength (A), elongation at break (B), tensile yield strength $(\mathrm{C})$, elongation at yield (D). See also Table 3. 
Table 3 also includes the properties of linear high molecular weight (HMW) PTMC and of selected elastomeric networks for comparison. The photo-crosslinked networks prepared from macromers with higher molecular weights show tensile properties comparable to those of linear non-crosslinked HMW-PTMC. Both types of materials are flexible with low E-moduli and high elongations at break, the values of the yield stress and yield strain are similar as well. The maximum tensile strength and elongation at break values of the networks prepared from macromers with molecular weights higher than approximately $20000 \mathrm{~g} / \mathrm{mol}$ surpass those of the linear high molecular weight PTMC polymers.

As Table 3 shows, these high values of maximum tensile strength and elongation at break lead to tough networks. While the toughness of the more densely crosslinked networks was rather low, the less densely crosslinked networks, prepared from macromers with molecular weights above $10000 \mathrm{~g} / \mathrm{mol}$, were quite tough. The toughness of these networks increased from approximately 1100 to $5500 \mathrm{~N} / \mathrm{mm}^{2}$ when the macromer molecular weight was increased for 20000 to $41000 \mathrm{~g} / \mathrm{mol}$. These values compare favourably with those of the linear polymers. For non-crosslinked high molecular weight PTMC, typical values of toughness range from 1000 to $3200 \mathrm{~N} / \mathrm{mm}^{2}$. (It should be noted that the linear HMW-PTMC 300000 strain hardens during tensile testing as a result of strain induced crystallization, see reference 5.)

The form stability of the PTMC networks was excellent. In cyclic tensile testing experiments, the rubber-like networks prepared from macromers with molecular weights of 10300 and $41000 \mathrm{~g} / \mathrm{mol}$ showed very low permanent deformations of 0.65 and $0.88 \%$, respectively, after 20 cycles to $50 \%$ elongation. Brittle networks obtained from the macromer with $\overline{\mathrm{M}}_{n}=3300 \mathrm{~g} / \mathrm{mol}$ failed prematurely upon cyclic stretching to $50 \%$ strain. When repeatedly elongated to a lower extension of $25 \%$, the network also showed a very low permanent deformation of $0.45 \%$. It is clear from Table 3 that the elasticity (and therefore the form stability) of the photo-crosslinked networks is much better than that of the linear high molecular weight PTMC specimens.

In comparison with PDMS and latex rubber, which are well-known elastomers used in the biomedical field, the tensile properties of photo-crosslinked PTMC are in the same order of magnitude: all materials are flexible, relatively strong, and elastic. The toughness and the resistance to permanent deformation of the rubber-like PTMC networks surpass that of PDMS and latex rubber. 
In surgical practice, implants are often sutured. For example, in vascular grafting or tissue engineering, grafts are sutured to the host vasculature. Also nerve guidance channels are sutured. Since punctures and defects are created during suturing, failure of the implanted structure can occur through crack propagation. The resistance to tearing of materials is experimentally determined by tear propagation strength and suture retention strength measurements.

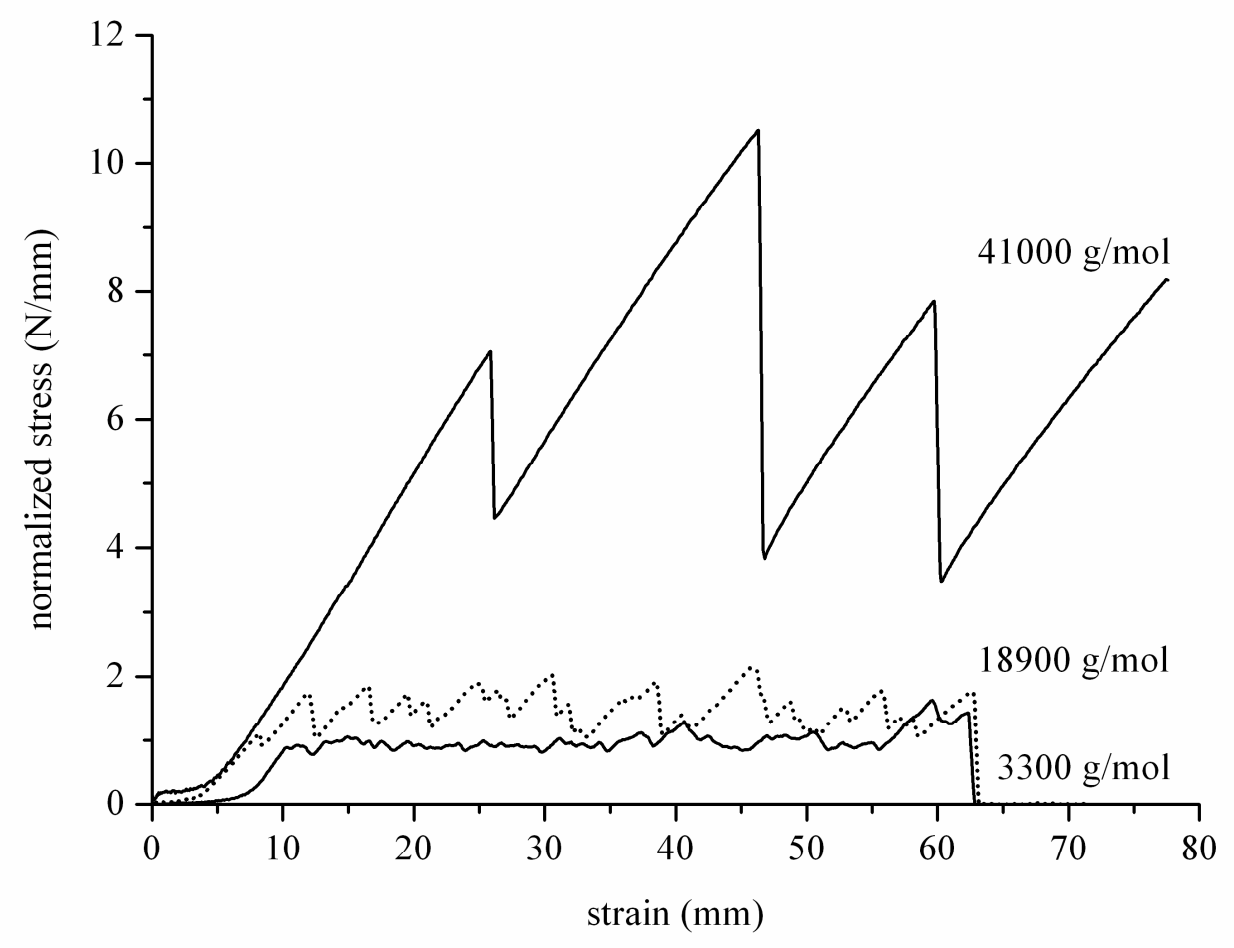

Figure 6. Strength versus strain diagrams determined during trouser TPS measurements of selected photo-crosslinked PTMC networks prepared from macromers of different molecular weights.

Figure 6 shows typical tear propagation curves in which the normalized stress is given as a function of strain for PTMC networks prepared from macromers with different molecular weights. The networks prepared from macromers of molecular weights below approximately $1800 \mathrm{~g} / \mathrm{mol}$ were too brittle to allow tear strength measurements in these trouser tearing tests. For networks prepared from macromers with higher $\overline{\mathrm{M}}_{n}$ values, the tear propagation strength increases with increasing molecular weight. This has been observed before for silicone rubbers. ${ }^{[4]}$ As is presented in Table 4, both the average and the maximum values of the tear propagation strength significantly increase above macromer molecular weights higher than $18900 \mathrm{~g} / \mathrm{mol}$ indicating that the propagation of the crack is 
increasingly restricted. In Figure 6, this is evident from the "stick-slip" tearing behavior of the network prepared from the macromer with $\overline{\mathrm{M}}_{n}=41000 \mathrm{~g} / \mathrm{mol}$. This network exhibits an average TPS of $7.1 \mathrm{~N} / \mathrm{mm}$, a maximum TPS of $11.1 \mathrm{~N} / \mathrm{mm}$ and a toughness of $420.6 \mathrm{~N}$.

Strain-crystallizing elastomers like natural rubber show "stick-slip" tearing behavior. ${ }^{[14]}$ Crack growth is restricted by crack-tip blunting at the interface of the formed crystallites. This toughening effect might also occur in the case of PTMC networks with high molecular weights between crosslinks, since linear high molecular weight PTMC undergoes strain-induced crystallization. ${ }^{[5]}$

Table 4. Values of the TPS, SRS and corresponding toughness ( $W_{T P S}$ and $W_{S R S}$, respectively) of extracted PTMC networks prepared by photo-crosslinking three-armed PTMC macromers of linear high molecular weight PTMC and of other relevant elastomeric networks. Triplicate measurements, values are averages \pm standard deviation.

\begin{tabular}{|c|c|c|c|c|c|}
\hline $\begin{array}{l}\overline{\mathrm{M}}_{n} \text { of } \\
\text { macromer }\end{array}$ & TPS $_{\text {average }}$ & TPS $_{\text {maximum }}$ & $\mathbf{W}_{\text {TPS }}$ & $\mathbf{S R S}_{\text {maximum }}$ & $\mathbf{W}_{\text {SRS }}$ \\
\hline $\mathrm{g} / \mathrm{mol}$ & $\mathbf{N} / \mathbf{m m}$ & $\mathbf{N} / \mathbf{m m}$ & $\mathbf{N}$ & $\mathbf{N} / \mathbf{m m}$ & $\mathbf{N}$ \\
\hline 1000 & $-{ }^{a}$ & $-{ }^{a}$ & $-{ }^{a}$ & $9.3 \pm 2.2^{b}$ & $5.9 \pm 3.1^{b}$ \\
\hline 1800 & $-{ }^{a}$ & $-{ }^{a}$ & $-{ }^{a}$ & $4.1 \pm 0.5^{b}$ & $8.0 \pm 0.9^{b}$ \\
\hline 3300 & $1.6 \pm 0.1^{\mathrm{c}}$ & $1.1 \pm 0.1^{\mathrm{c}}$ & $51.6 \pm 3.8^{c}$ & $3.3 \pm 0.5$ & $9.6 \pm 3.5$ \\
\hline 10300 & n.d. & n.d. & n.d. & $5.0 \pm 0.6$ & $23.3 \pm 4.3$ \\
\hline 18900 & $1.4 \pm 0.1$ & $2.1 \pm 0.1$ & $78.1 \pm 5.8$ & $4.7 \pm 1.0$ & $17.6 \pm 6.1$ \\
\hline 20000 & $1.8 \pm 0.1$ & $2.5 \pm 0.3$ & $96.9 \pm 9.4$ & $5.5 \pm 0.5$ & $23.4 \pm 5.5$ \\
\hline 24600 & $3.0 \pm 0.4$ & $4.9 \pm 0.9$ & $159.2 \pm 9.9$ & $7.7 \pm 1.3$ & $41.1 \pm 13.8$ \\
\hline 41000 & $7.1 \pm 1.0$ & $11.1 \pm 1.5$ & $420.6 \pm 38.2$ & $13.9 \pm 3.8$ & $162.0 \pm 105.2$ \\
\hline$H M W 243000^{\mathrm{c}}$ & $-{ }^{d}$ & $18.7 \pm 6.7$ & $1039.8 \pm 880.5$ & $37.3 \pm 0.9$ & $6293.8 \pm 706.7$ \\
\hline PDMS & $0.16 \pm 0.01$ & $0.19 \pm 0.01$ & $8.4 \pm 0.2$ & $1.3 \pm 0.1$ & $2.5 \pm 0.5$ \\
\hline Latex rubber & $-{ }^{e}$ & $-{ }^{e}$ & $-{ }^{e}$ & $30.0 \pm 4.8$ & $3056.8 \pm 547.9$ \\
\hline
\end{tabular}

${ }^{a}$ Networks too brittle to allow tear strength measurements.

${ }^{\mathrm{b}}$ Cracks already form when perforating the specimens.

${ }^{\mathrm{c}} \mathrm{n}=2$.

${ }^{\mathrm{d}}$ Stress continues to increase upon elongation, average value was not determined.

e Specimen did not tear.

n.d.: not determined. 
Table 4 indicates that the increased resistance to tearing as a function of macromer molecular weight and the corresponding toughness ( $\mathrm{W}_{\mathrm{TPS}}$ ) correlate well with the toughness of non-punctured networks in tensile tests, which are shown in Table 3.

Most often suture retention strength values are reported in the development of vascular grafts. For the networks prepared from macromers of different molecular weights, the normalized maximum stresses determined during the tearing of a suture through the material are given in Table 4. With increasing macromer molecular weight, the maximum SRS first decreased before increasing again. Although networks prepared from macromers with molecular weights below approximately $1800 \mathrm{~g} / \mathrm{mol}$ had relatively high SRS values, the materials were quite brittle and cracks already formed when the specimens were perforated to insert the suture. The toughness of the networks, determined as the area under the stress-strain curves in the suture retention strength measurements, continuously increased with increasing molecular weight of the corresponding macromers.

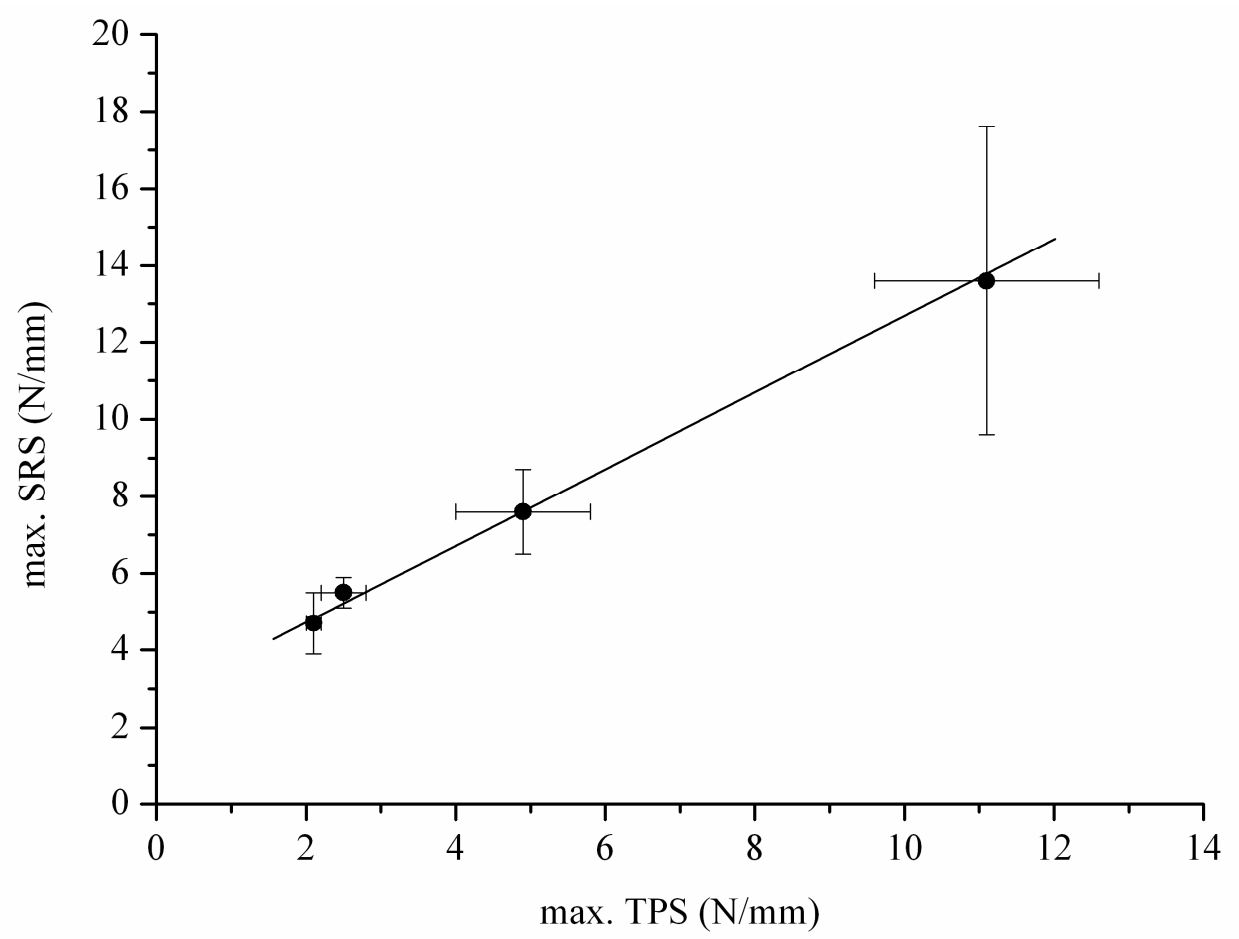

Figure 7. Values of the maximum SRS plotted against the maximum TPS of PTMC networks obtained by photo-crosslinking that show rubber-like tensile behavior. 
Figure 7 indicates that there is a linear relationship between the TPS and the maximum SRS of photo-crosslinked PTMC networks obtained from macromers with $\overline{\mathrm{M}}_{n}$ values higher than $18900 \mathrm{~g} / \mathrm{mol}$. These are the networks that display rubber-like tensile behavior (Figure 4).

The network obtained from the PTMC macromer with molecular weight $41000 \mathrm{~g} / \mathrm{mol}$ showed excellent tear resistance with high maximum SRS values $(13.9 \mathrm{~N} / \mathrm{mm})$ and high toughness values $\left(\mathrm{W}_{\mathrm{SRS}}=162.0 \mathrm{~N}\right)$. For comparison, Table 4 also includes the determined values for high molecular weight PTMC and selected elastomeric networks. Although poly(dimethylsiloxane) is flexible and elastic, it tears with extreme ease. All photo-crosslinked PTMC networks have significantly higher values of tear propagation strength, suture retention strength and corresponding toughness values. Latex rubber is also significantly tougher than PDMS, its maximum SRS was $30.0 \pm 4.8 \mathrm{~N} / \mathrm{mm}$ with a high toughness of $3056.8 \mathrm{~N}$ and in the tear propagation determinations the specimens did not even tear. Non cross-linked, linear HMW-PTMC ( $\left.\overline{\mathrm{M}}_{n}=243000 \mathrm{~g} / \mathrm{mol}\right)$ has very high maximum TPS and maximum SRS (values are 18.7 \pm 6.7 and $37.3 \pm 0.9 \mathrm{~N} / \mathrm{mm}$, respectively). Also the corresponding values of the toughness (1039.8 and $6293.8 \mathrm{~N}$, respectively), are very high. As previously indicated, this can be due to strain-induced crystallization. Its tearing properties are equivalent to those of latex rubber.

\section{Mechanical Properties of Native Blood Vessels}

The excellent creep resistance, good tensile, and good tear properties would make photo-crosslinked PTMC networks very well-suited for application as nerve guidance channels, vascular grafts or materials for use in vascular tissue engineering. Ideally, the mechanical properties of the implanted biomaterials are in accordance with those of the native tissues.

For comparison with our PTMC networks, we also determined the tensile and tear properties of different porcine and ovine blood vessels. The values of the tensile properties are presented in Table 5. For these vessels, the E-modulus values at low strains (Young's modulus) vary between 0.05 and $0.12 \mathrm{MPa}$. In the high strain region, these values are higher and vary between 1.44 and 3.41 MPa. Human blood vessels are quite similar with Emoduli of 1 to $3 \mathrm{MPa} .{ }^{[19]}$ The maximum tensile strengths of native porcine and ovine blood vessels were 0.8 to $2.4 \mathrm{~N} / \mathrm{mm}^{2}$, with elongations at break of 75 to $145 \%$. Their toughness 
determined in tensile testing experiments was 24 to $117 \mathrm{~N} / \mathrm{mm}^{2}$. The differences in mechanical properties between the different animals were not significant. Comparing these tensile testing results with those of the different photo-crosslinked PTMC networks presented in Table 3, it can be seen that the tensile strength, elongation at break and toughness of PTMC networks prepared from macromers with molecular weights higher than approximately $10000 \mathrm{~g} / \mathrm{mol}$ surpass those of the blood vessels. The values for the elasticity modulus of the networks are somewhat higher. When preparing porous structures from these materials these values and those of the tensile strength will decrease with increasing porosity.

Table 5. Tensile properties of different porcine and ovine blood vessels. Values are reported as averages \pm standard deviation.

\begin{tabular}{|c|c|c|c|c|}
\hline \multirow{2}{*}{ blood vessel } & \multirow{2}{*}{$\frac{\text { E-Modulus }}{\text { MPa }}$} & \multirow{2}{*}{$\frac{\sigma_{\max }}{\mathrm{N} / \mathbf{m m}^{2}}$} & \multirow{2}{*}{$\frac{\varepsilon_{\max }}{\%}$} & \multirow{2}{*}{$\frac{\mathbf{W}^{\mathrm{c}}}{\mathbf{N} / \mathbf{m m}^{\mathbf{2}}}$} \\
\hline & & & & \\
\hline porcine carotid artery $^{a}$ & $\begin{array}{c}0.05 \pm 0.01 \\
(2.24 \pm 0.26)^{b}\end{array}$ & $1.0 \pm 0.1$ & $139 \pm 18$ & $54.1 \pm 35.0$ \\
\hline porcine aorta $^{\mathrm{d}}$ & $\begin{array}{c}0.12 \pm 0.03 \\
(1.44 \pm 0.87)^{\mathrm{e}}\end{array}$ & $0.8 \pm 0.3$ & $75 \pm 15$ & $24.0 \pm 9.4$ \\
\hline ovine carotid artery ${ }^{\mathrm{f}}$ & $\begin{array}{c}0.11 \pm 0.02 \\
(3.41 \pm .55)^{b}\end{array}$ & $2.4 \pm 1.4$ & $145 \pm 14$ & $117.0 \pm 65.3$ \\
\hline \multicolumn{5}{|c|}{$\begin{array}{l}{ }^{\mathrm{a}} \text { Diameter }=5.4 \pm 0.4 \mathrm{~mm} \text {, wall thickness }=1.0 \pm 0.2 \mathrm{~mm}, \mathrm{n}=3 \text {. } \\
{ }^{\mathrm{b}} \text { E-modulus value at } 100-110 \% \text { elongation. } \\
{ }^{\mathrm{c}} \text { Normalized area under the stress-strain curve }(\mathrm{W}, \text { toughness). } \\
\mathrm{d} \text { Specimens (width }=4.6 \pm 0.5 \mathrm{~mm} \text {, thickness }=1.6 \pm 0.3 \mathrm{~mm}, \mathrm{n}=4) \text { were cut out from } 12 \mathrm{~cm} \text { long porcine aortas } \\
\text { with diameters of approximately } 30 \mathrm{~mm} \text {. } \\
{ }^{\mathrm{e}} \text { E-modulus value at } 40-45 \% \text { elongation. } \\
{ }^{\mathrm{f}} \text { Diameter }=5.1 \pm 0.3 \mathrm{~mm} \text {, wall thickness }=0.5 \pm 0.1 \mathrm{~mm}, \mathrm{n}=6 \text {. }\end{array}$} \\
\hline
\end{tabular}

Regarding the tearing strength and suture retention strength of the animal blood vessels, Table 6 shows that the determined suture retention strengths of the carotid arteries varied between 3.9 and $9.6 \mathrm{~N} / \mathrm{mm}$. The corresponding toughness values varied between 51 and $108 \mathrm{~N}$. The suture retention strength and toughness of the porcine aorta $(2.2 \mathrm{~N} / \mathrm{mm}$ and 16.9 N, respectively) were somewhat lower than of the other blood vessels. This can be due to the longitudinal orientation of the tissue of the aorta specimens. Tear resistance strengths 
could only be determined for porcine aortas. The maximum TPS of the porcine aortas was $3.0 \mathrm{~N} / \mathrm{mm}$, with a toughness (determined as the area under the stress-strain curve) of $177 \mathrm{~N}$. The data presented in Table 4, shows that photo-crosslinked PTMC networks prepared from macromers with the highest molecular weights (higher than $20000 \mathrm{~g} / \mathrm{mol}$ ) are more resistant to tearing than the natural tissues. This implies that these networks can readily be sutured to natural blood vessels.

Table 6. Values of the maximum tear propagation strength, maximum suture retention strength and corresponding toughness ( $W_{T P S}$ and $W_{S R S}$, respectively) of different porcine and ovine blood vessels. Values are reported as averages \pm standard deviation.

\begin{tabular}{|c|c|c|c|c|c|}
\hline \multirow{2}{*}{ blood vessel } & TPS $_{\text {average }}$ & TPS $_{\text {maximum }}$ & $\mathbf{W}_{\mathrm{TPS}}{ }^{\mathrm{a}}$ & $\mathbf{S R S}_{\text {maximum }}$ & $\mathbf{W}_{\mathrm{SRS}}{ }^{\mathrm{a}}$ \\
\hline & $\mathbf{N} / \mathbf{m m}$ & $\mathbf{N} / \mathbf{m m}$ & $\mathbf{N}$ & $\mathbf{N} / \mathbf{m m}$ & $\mathbf{N}$ \\
\hline porcine carotid artery ${ }^{b}$ & n.d. & n.d. & n.d. & $5.8 \pm 3.2$ & $62.3 \pm 21.5$ \\
\hline porcine aorta $^{c}$ & $2.7 \pm 1.0$ & $3.0 \pm 1.1$ & $177.3 \pm 62.1$ & $2.2 \pm 0.5$ & $16.9 \pm 6.5$ \\
\hline ovine carotid artery ${ }^{\mathrm{d}}$ & n.d. & n.d. & n.d. & $7.7 \pm 1.6$ & $108.1 \pm 47.5$ \\
\hline
\end{tabular}

\footnotetext{
${ }^{\mathrm{a}}$ Normalized area under the stress-strain curve in tear propagation strength measurement $\left(\mathrm{W}_{\mathrm{TPS}}\right)$ or suture retention strength measurements $\left(\mathrm{W}_{\mathrm{SRS}}\right)$.

${ }^{\mathrm{b}}$ Diameter $=5.6 \pm 1.3 \mathrm{~mm}$, wall thickness $=0.9 \pm 0.1 \mathrm{~mm}, \mathrm{n}=6$.

${ }^{\mathrm{c}}$ Specimens with a thickness of $2.1 \pm 0.2 \mathrm{~mm}$ were cut out from $12 \mathrm{~cm}$ long porcine aortas with diameters of approximately $30 \mathrm{~mm}$. In TPS measurements the width was $20.8 \pm 0.8 \mathrm{~mm}, \mathrm{n}=3$ and in SRS measurements the width was $9.8 \pm 0.4 \mathrm{~mm}, \mathrm{n}=4$.

${ }^{\mathrm{d}}$ Diameter $=5.1 \pm 0.2 \mathrm{~mm}$, wall thickness $=1.0 \pm 0.1 \mathrm{~mm}, \mathrm{n}=3$.

n.d.: not determined.
}

\section{Conclusions}

Photo-crosslinked networks were readily prepared from PTMC oligomers endfunctionalized with methacrylate end groups. While all networks were creep resistant, other mechanical properties of the obtained networks depended on the molecular weight of the macromer from which they are prepared. Networks prepared from PTMC macromers with molecular weights lower than $1800 \mathrm{~g} / \mathrm{mol}$ were relatively rigid and brittle. Networks prepared from macromers with molecular weights higher than approximately $10000 \mathrm{~g} / \mathrm{mol}$ were rubber-like, with E-modulus values close to $5 \mathrm{MPa}$. Maximum tensile strength and elongation at break values increased with increasing macromer molecular weight. Networks 
prepared from PTMC macromers with molecular weight $40000 \mathrm{~g} / \mathrm{mol}$ had a yield strength of $1.7 \mathrm{MPa}$ and a maximum tensile strength of $17 \mathrm{MPa}$. The elongation at break was more than $1200 \%$.

PTMC networks prepared from macromers of molecular weights higher than approximately $10000 \mathrm{~g} / \mathrm{mol}$ were shown to be tough and tear resistant as well. Tear propagation strength and suture retention strength measurements indicated that photo-crosslinked networks prepared from PTMC macromers with molecular weights higher than approximately $10000 \mathrm{~g} / \mathrm{mol}$ are more resistant to tearing than porcine and ovine blood vessels.

Also, in comparison with well-known elastomers like PDMS and latex natural rubber, it is shown that the strength and toughness of PTMC networks prepared from macromers of higher molecular weights is superior.

\section{References}

[1] Pourdeyhimi B, Kern S. Ease of suturing surgical fabrics - a quantitative-evaluation. Am J Surg 1985, 149, 387-389.

[2] Pourdeyhimi B, Wagner D. On the correlation between the failure of vascular grafts and their structural and material properties - a critical analysis. J Biomed Mater Res 1986, 20, 375-409.

[3] Greenwald SE, Berry CL. Improving vascular grafts: the importance of mechanical and haemodynamic properties. J Pathol 2000, 190, 292-299.

[4] Bhowmick AK. Tear strength of elastomers over a range of rates, temperatures and cross-linking - tearing energy-spectra. J Mater Sci 1986, 21, 3927-3932.

[5] Pego AP, Grijpma DW, Feijen J. Enhanced mechanical properties of 1,3-trimethylene carbonate polymers and networks. Polymer 2003, 44, 6495-6504.

[6] Bat E, Plantinga JA, Harmsen MC, van Luyn MJA, Zhang Z, Grijpma DW, Feijen J. Trimethylene carbonate and epsilon-caprolactone based (co)polymer networks: mechanical properties and enzymatic degradation. Biomacromolecules 2008, 9, 32083215.

[7] Song Y, Kamphuis MMJ, Zhang Z, Sterk LMT, Vermes I, Poot AA, Feijen J, Grijpma DW. Flexible and elastic porous poly(trimethylene carbonate) structures for use in vascular tissue engineering. Acta Biomater 2010, 6, 1269-1277.

[8] Storey RF, Warren SC, Allison CJ, Puckett AD. Methacrylate-endcapped poly(d,1lactide-co-trimethylene carbonate) oligomers. Network formation by thermal freeradical curing. Polymer 1997, 38 , 6295-6301.

[9] Matsuda T, Mizutani M. Liquid acrylate-endcapped biodegradable poly(ecaprolactone-co-trimethylene carbonate). II. Computer-aided stereolithographic microarchitectural surface photoconstructs. J Biomed Mater Res 2002, 62, 395-403.

[10] Crawford ED, Lesser AJ. Brittle to ductile: fracture toughness mapping on controlled epoxy networks. Polym Eng Sci 1999, 39, 385-392. 
[11] Flipsen TAC, Derks R, van der Vegt H, Stenekes R, Pennings AJ, Hadzhiioannou G. Densely crosslinked polycarbosiloxanes. II: Thermal and mechanical properties. $J$ Polym Sci B: Polym Phys 1997, 35, 1311-1331.

[12] Smith TL. Strength of elastomers - perspective. Polym Eng Sci 1977, 17, 129-143.

[13] Mark JE. Improved elastomers through control of network chain-length distributions. Rubber Chem Technol 1999, 72, 465-483.

[14] Thomas AG. The development of fracture-mechanics for elastomers. Rubber Chem Technol 1994, 67, G50-G60.

[15] Hou QP, Grijpma DW, Feijen J. Creep-resistant elastomeric networks prepared by photocrosslinking fumaric acid monoethyl ester-functionalized poly(trimethylene carbonate) oligomers. Acta Biomater 2009, 5, 1543-1551.

[16] Zhang Z. Biodegradable Block Copolymers Based on Trimethylene Carbonate, Lactides, and Poly(Ethylene Glycol). PhD Thesis, University of Twente, 2006.

[17] Carothers WH, van Natta FJ. Glycol esters of carbonic acid. J Am Chem Soc 1930, 52, 314-326.

[18] Zhu KJ, Hendren RW, Jensen K, Pitt CG. Synthesis, properties, and biodegradation of poly(1,3-trimethylene carbonate). Macromolecules 1991, 24, 1736-1740.

[19] Uchida T, Ikeda S, Oura H, Tada M, Nakano T, Fukuda T, Matsuda T, Negoro M, Arai F. Development of biodegradable scafolds based on patient-specific arterial configuration. J Biotechnol 2008, 133, 213-218. 


\title{
Chapter 4
}

\section{Microstructured Photo-Crosslinked Poly(Trimethylene Carbonate) for Use in Soft Lithography Applications: \\ Biodegradable Structures as Alternatives for Poly(Dimethylsiloxane)}

\begin{abstract}
Photo-crosslinkable poly(trimethylene carbonate) (PTMC) macromers were used in the fabrication of microstructured surfaces. Microstructured PTMC surfaces were obtained within minutes by hot embossing the macromer against structured silicon masters, followed by photo-crosslinking, resulting in network formation. The microstructures of the master could be precisely replicated with shrinkage of the structures being less than $5.7 \%$. The microstructured PTMC was investigated for use in two different applications: as stamping material to transfer a model protein to another surface and as structured substrate for cell culture. Applying the flexible and elastic materials as stamps, a model protein (bovine serum albumin labelled with fluorescein isothiocyanate) was successfully patterned on glass surfaces. In addition, in cell culturing experiments, the behavior of human mesenchymal stem cells on non-structured and microstructured PTMC surfaces was investigated. The cells adhered strongly to the PTMC surfaces and proliferated well. In comparison to poly(dimethylsiloxane) (PDMS), which is commonly used in soft lithography, (microstructured) PTMC networks offer significant advantages. They not only show better compatibility with cells, but they are also biodegradable and have significantly better mechanical properties. Both materials are transparent, flexible and elastic at room temperature, but the resistance to tearing is much higher for PTMC networks than for PDMS. For these reasons PTMC might be an alternative material to poly(dimethylsiloxane) in the fields of biology, medicine, and tissue engineering, where microfabricated devices are increasingly being applied.
\end{abstract}




\section{Introduction}

Microfabrication has made a large impact on biology and medicine since it allows conducting controlled experiments at the micrometer scale. Envisaged applications include medical devices such as biosensors and tools for biochemistry, molecular biology, and cell biology, which are being developed to allow quantitative measurements at the cellular level. ${ }^{[1,2]}$

Soft lithography describes a set of technologies with which microstructures can be created on (soft) materials by printing, molding, or embossing using an elastomer with a patterned surface as a mask, stamp or mold. ${ }^{[3,4]}$ Poly(dimethylsiloxane) (PDMS) has emerged as the key material in soft lithography as its properties are suitable for many applications and, perhaps even more importantly, it is well known and readily available commercially.

In the biomedical field, surface structured PDMS stamps are increasingly being applied in microcontact printing $(\mu \mathrm{CP})$. The technique was first developed to transfer thiol-patterns onto gold surfaces, but is now being used to transfer a variety of inks, including proteins and other biomolecules. ${ }^{[5,6]}$ The range of substrate materials that are being patterned has also expanded significantly. ${ }^{[7,8]}$ Although other polymers and hydrogels have been investigated as alternatives, PDMS is still by far the most widely used stamp material due to specific properties that make it well suited for $\mu \mathrm{CP}$ : it is optically transparent, flexible and elastic to allow conformal contact with all kinds of surfaces. ${ }^{[9]}$ Microstructured PDMS has also been used to prepare microfluidic devices. ${ }^{[10]}$ Although these devices were initially developed as analytical tools and devices to investigate the effect of microstructure dimensions on the biological response of cells, they are nowadays also being considered as implantable devices, and even as scaffolding structures in tissue engineering. ${ }^{[10-14]}$ However, PDMS might not be the best material for these applications as it has been shown to have limited biocompatibility with cells in long term culture, and it is highly resistant to degradation. ${ }^{[15-18]}$ Furthermore, PDMS is difficult to process into porous cell scaffolding structures. $^{[18]}$

From this it follows that for several applications in the biomedical field, there is a need for alternative materials to PDMS that are flexible and allow accurate replication of a microstructures from silicon masters.

In earlier work we have investigated the properties of photo-crosslinked poly(trimethylene carbonate) networks prepared from different molecular weight methacrylate-functionalized PTMC oligomers. These networks are flexible, elastic materials that are amorphous and 
transparent with low glass transition temperatures. Their mechanical properties depend on the molecular weight of the macromers used. ${ }^{[19]}$ Also, these networks were shown to be compatible with different cell types and degraded in vivo by a surface erosion process. ${ }^{[20-22]}$ While the preparation of PDMS networks is very time consuming, as the thermal curing process can take hours to complete, photo-crosslinking is a very rapid process, allowing the solidification step to be conducted in minutes. ${ }^{[19,23-25]}$

In this work we aimed at investigating the use of photo-crosslinked PTMC as an alternative to PDMS in microfabrication applications. The microstructures present on silicon masters are replicated into the surface of different PTMC resins by casting or hot embossing, followed by photo-crosslinking to form networks.

\section{Materials and Methods}

\section{Materials}

PDMS networks (Sylgard 184, Dow Corning, USA) were prepared according to manufacturers specifications. PDMS base material and curing agent were thoroughly mixed in a 10:1 weight ratio, degassed under vacuum and cured overnight at $80{ }^{\circ} \mathrm{C}$.

Three-armed methacrylated PTMC macromers with molecular weights between 3500 and $41000 \mathrm{~g} / \mathrm{mol}$ were synthesized as described previously. ${ }^{[19]}$ Briefly, hydroxyl-terminated PTMC oligomers were prepared by ring-opening polymerization (oligomerization) of polymer grade 1,3-trimethylene carbonate (TMC, Boehringer Ingelheim, Germany) using glycerol as initiator and $0.07 \mathrm{wt} \%$ stannous octoate ( SnOct $_{2}$, Sigma, USA) as catalyst. Glycerol (99.5+\% spectrophotometric grade, Aldrich, USA) was dehydrated by briefly heating at $180^{\circ} \mathrm{C}$. By varying the monomer-to-initiator ratio, three-armed oligomers with varying molecular weights could be prepared.

Crosslinkable PTMC macromers were then obtained by reaction of the hydroxyl-terminated oligomers with methacryloyl chloride (Fluka, Germany) in the presence of triethyl amine (Fluka, Germany), or with methacrylic anhydride (Aldrich, Germany). To prevent premature crosslinking, $0.06 \mathrm{wt} \%$ hydroquinone (Aldrich, Germany) and $0.06 \mathrm{wt} \%$ $( \pm)$ - $\alpha$-tocopherol (Fluka, Switzerland) were used as inhibitors. The PTMC macromers were purified by precipitation in ethanol or by distillation of the excess methacrylic anhydride, followed by drying under vacuum. 
The monomer conversions in the oligomerization reactions, the molecular weights and the degrees of functionalization of the three-armed oligomers were determined by ${ }^{1} \mathrm{H}-\mathrm{NMR}$ spectroscopy (Varian Inova $300 \mathrm{MHz}$, USA), using $\mathrm{CDCl}_{3}$ (Merck, Germany) as solvent. PTMC macromers with number average molecular weights of $\overline{\mathrm{M}}_{n}=3500,25400$ and $41000 \mathrm{~g} / \mathrm{mol}$ were prepared. These macromers are designated as PTMC 3500, PTMC 25400 and PTMC 41000, respectively.

Different resins containing PTMC macromer and a photoinitiator (Irgacure 2959 from Ciba Specialty Chemicals, Switzerland or Lucirin TPO-L from BASF, Germany) were formulated. The macromer with the lowest molecular weight PTMC 3500 was a viscous liquid at room temperature and could be readily mixed with $2.5 \mathrm{wt} \%$ (relative to the macromer) of the photo-initiator Irgacure 2959, that was dissolved in a minor amount of chloroform (Chemopack, Belgium). The chloroform was allowed to evaporate before use. PTMC macromers with molecular weights higher than approximately $10000 \mathrm{~g} / \mathrm{mol}$ are solids at room temperature. ${ }^{[19]}$ PTMC 25400 was mixed with $0.1 \mathrm{wt} \%$ of the photo-initiator Lucirin TPO-L at $80{ }^{\circ} \mathrm{C}$, while PTMC 41000 needed to be dissolved in a small amount of chloroform to be able to mix it with $2.5 \mathrm{wt} \%$ Irgacure 2959 at room temperature. In the latter case, the chloroform was allowed to evaporate before use.

\section{Preparation of Microstructured PDMS and PTMC Surfaces by Replication}

Two different silicon wafers (master A and master B) having channels and ridges of different dimensions were manufactured by standard cleanroom lithography and etching techniques. These masters were prepared according to Figure 1.

The widths of the channels (b) and ridges (a) and the height of the ridges (c) of the silicon masters were determined from scanning electron microscopy images. The respective measurements were 34.5, 16.9 and $54.5 \mu \mathrm{m}$ for master A, and 51.1, 315.3 and $52.2 \mu \mathrm{m}$ for master B. Upon replication of these wafers, PDMS and PTMC replicas (see below) will have the inverse structure of the master template; master A was designed to yield microstructured surfaces with narrow channels $(16.9 \mu \mathrm{m})$, while master $\mathrm{B}$ will give microstructured surfaces with much broader channels of $315.3 \mu \mathrm{m}$. To allow easy removal of the PDMS and PTMC replicas, the microstructured silicon masters were coated with a monolayer of hydrophobic perfluorooctyl-trichlorosilane (FOTS, Aldrich, USA). 

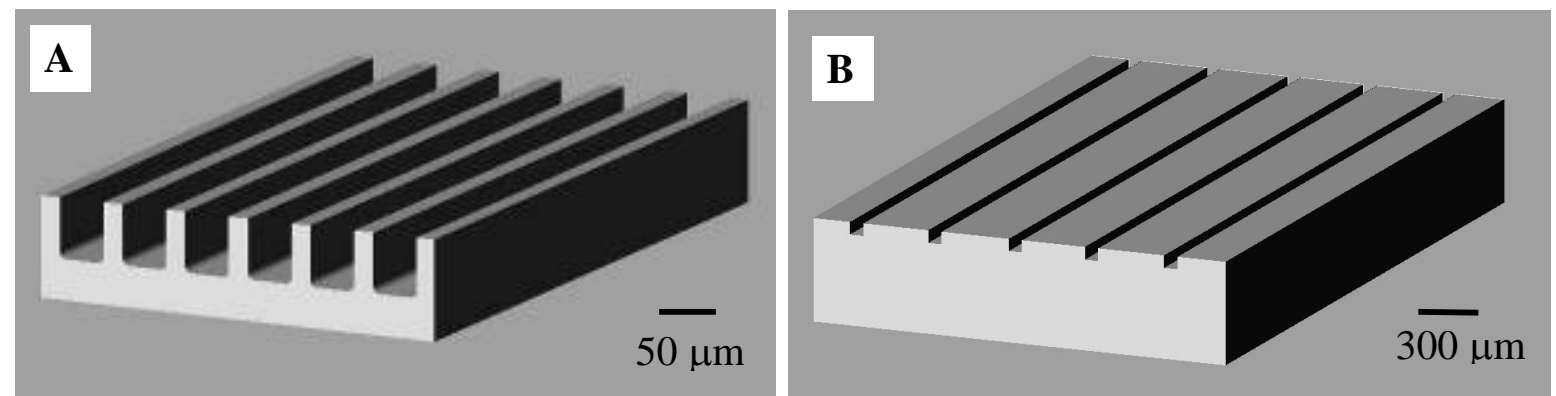

Figure 1. Designs showing the dimensions of the features of silicon masters used to prepare microstructured PDMS and PTMC surfaces by replication. Master A (left) yields replicas with narrow channels, while master B (right) yields replicas with broad channels. Note the different size scales.

In soft lithography and replica molding, PDMS is the most often used polymer material. To prepare microstructured PDMS surfaces, the liquid mixture of PDMS base and curing agent were cast on the silicon masters at a thickness of approximately $400 \mu \mathrm{m}$, cured at $80{ }^{\circ} \mathrm{C}$ for $16 \mathrm{~h}$ temperatures, and carefully released.

To fabricate microstructured PTMC surfaces, the formulated PTMC resins were applied on the surface of the silicon masters and cured by photo-crosslinking. The resin based on PTMC 3500 was liquid at room temperature and could be cast directly on the silicon master at thicknesses of 250 to $400 \mu \mathrm{m}$. Photo-crosslinking was then done in a crosslinking cabinet (Ultralum) under nitrogen flow at room temperature for $15 \mathrm{~min}$ at $365 \mathrm{~nm}$. The distance from the lamp to the sample was approximately $10 \mathrm{~cm}$. The microstructured solid PTMC films that formed could be easily peeled off. The resins based on PTMC 25400 and PTMC 41000 were solids at room temperature, and were hot embossed against the silicon templates for $2 \mathrm{~min}$ at $120{ }^{\circ} \mathrm{C}$ applying a pressure of 40 bar using a hydraulic press (Specac). The resin-covered master was immediately placed under a Xe-lamp with wavelength of 300-400 nm (Lightningcure LC8, Hamamatsu) and crosslinked for 5 min. The distance of the lamp to the sample was approximately $20 \mathrm{~cm}$. The microstructured PTMC films could easily be detached from the masters. Their thicknesses ranged from 180 to $250 \mu \mathrm{m}$.

The microstructured surfaces and the cross-sections of the prepared PDMS and PTMC replicas were gold-sputtered and analysed by scanning electron microscopy (SEM, Philips XL 30 ESEM-FEG). 
A non-structured flat silicon wafer was also used to prepare non-patterned PDMS and PTMC network films for use in cell culturing experiments (see below).

\section{Protein Stamping Using a Microstructured PTMC Stamp}

The use of microstructured PTMC materials as stamps in protein patterning experiments on glass was evaluated using bovine serum albumin labelled with fluorescein isothiocyanate (BSA/FITC from Sigma, USA) as model protein. The PTMC 25400 based resin was hot embossed on silicon master $\mathrm{B}$ and photo-cured as described before to yield a microstructured material. Specimens measuring approximately $7 \times 10 \mathrm{~mm}^{2}$ were cut out and inked with a $50 \mu \mathrm{g} / \mathrm{ml}$ solution of BSA/FITC (Sigma, USA) in phosphate buffered saline (PBS) at $37{ }^{\circ} \mathrm{C}$ for 30 and $60 \mathrm{~min}$. The excess solution was removed, and the microstructured PTMC specimens were brought into contact with the glass surfaces to allow protein transfer to occur. Gentle pressure was applied at contact times of 5, 10 and $30 \mathrm{~min}$. The patterns on the glass surfaces were subsequently analysed by confocal laser scanning microscopy (LEICA TCS SP2).

Note that the PTMC stamps were used as prepared, without additional purification or surface treatments.

\section{Cell Culturing on (Microstructured) PDMS and PTMC Surfaces}

To investigate the response of cells to the PDMS and PTMC surfaces, human mesenchymal stem cells (hMSC) were seeded and cultured on non-structured, as well as on microstructured (replicates of master A) surfaces. The specimens were cut out of microstructured films, measuring approximately $7 \times 10 \mathrm{~mm}^{2}$ and were used without further purification. The PTMC specimens were prepared from PTMC 25400 by hot embossing and subsequent photo-crosslinking.

Human MSCs were isolated and proliferated as described previously. ${ }^{[26]}$ Bone marrow aspirates $(5-20 \mathrm{ml})$ were obtained from donors with written informed consent. Briefly, aspirates were re-suspended using $20 \mathrm{G}$ needles, plated at a density of $5 \times 10^{5} \mathrm{cell} / \mathrm{s} / \mathrm{cm}^{2}$ and cultured in hMSC proliferation medium which consists of $\alpha$-MEM, $10 \%$ FBS, $0.2 \mathrm{mM}$ Asap, $2 \mathrm{mM}$ L-glutamine, $100 \mathrm{U} / \mathrm{ml}$ penicillin, $10 \mu \mathrm{g} / \mathrm{ml}$ streptomycin and $1 \mathrm{ng} / \mathrm{ml}$ basic fibroblast growth factor (bFGF, Instruchemie, The Netherlands). Cells were grown in cell culturing flasks at $37{ }^{\circ} \mathrm{C}$ in a humidified atmosphere with $5 \% \mathrm{CO}_{2}$. After two days the 
medium was changed and non-adherent cells removed. The medium was refreshed twice a week until cells reached confluence. Upon confluence, the cells were trypsinized with $0.25 \%$ trypsin containing $1 \mathrm{mM}$ EDTA for $5 \mathrm{~min}$ at $37{ }^{\circ} \mathrm{C}$ and counted. The PDMS and PTMC materials were seeded with $3 \times 10^{4}$ cells $/ 100 \mu$ l of medium and incubated overnight to allow adherence, after which $3 \mathrm{ml}$ of culture medium were carefully added. Cell culturing was continued for 7 days, the culture medium was refreshed twice. Cell culturing experiments were performed in duplicate.

All specimens were analysed at 1, 3 and 7 days of culture. At each time point, cell adhesion, cell distribution and cell morphology were assessed by light microscopy using methylene blue staining and by scanning electron microscopy (SEM). The specimens were removed from culture, carefully washed with PBS and fixated with $1.5 \%(\mathrm{v} / \mathrm{v})$ glutaraldehyde (AGA, Sigma) in $0.14 \mathrm{M}$ cacodylic acid buffer (Sigma) pH 7.3 for $30 \mathrm{~min}$. The specimens were then carefully washed several times with PBS.

For SEM, the specimens were dehydrated by sequential immersion in ethanol solutions of $50,60,70,80,90$, and 100\% v/v water. The samples were kept in absolute alcohol and taken to the critical point, using $\mathrm{CO}_{2}$. Before SEM analysis the dried specimens were sputter-coated with a thin layer of gold.

\section{Results and Discussion}

\section{Replication of Microstructures on PDMS Surfaces}

PDMS elastomers have emerged as key materials in soft lithography and microfabrication. ${ }^{[10]}$ Although very much used because of its inert nature, flexibility and elasticity, transparency, and ease of processing, PDMS has some serious drawbacks: unless it is reinforced, the mechanical properties of PDMS are quite poor. As reported in earlier work using Sylgard 184, even though the materials is elastic, has a tensile strength of 8.7 $\mathrm{N} / \mathrm{mm}^{2}$, an E-Modulus of $2.6 \mathrm{MPa}$ and an elongation at break of $164 \%$, it is very brittle with extremely low resistance to tearing (see Table 3 below). ${ }^{[19]}$ This brittleness follows from the very low values of average tear propagation strengths and maximum suture retention strengths, being only $0.19 \mathrm{~N} / \mathrm{mm}$ and $1.3 \mathrm{~N} / \mathrm{mm}$, respectively. Resistance to tearing is very important since defects created for example by punching inlets and outlets in microfluidic devices can lead to tearing and catastrophic failure of the device. When 
microstructured PDMS is used as an in vivo implant or in tissue engineering applications, the devices are repeatedly exposed to stresses and can fail prematurely. ${ }^{[10]}$ In addition, PDMS is a non-degradable material that sometimes has to be removed from the body.

For reasons of comparison, microstructured PDMS materials were first prepared by casting onto silicon masters and thermal curing. Figure 2 shows SEM images of the top view and a cross-section of microstructured PDMS, replicated using master A. Note the cracks which are present in the corners of the channels, which are most likely formed due to the brittle nature of the polymer network. The dimensions of the microstructures obtained on the PDMS surfaces are listed in Table 1. It can be seen that, although shrinkage occurs, the dimensions closely resemble those of the masters used in the replication process. We calculated the shrinkage by comparing the sums of the channel and ridge widths of the microstructured replicas and the patterned silicon wafers. The channel and ridge widths were determined by SEM. The shrinkage was found to be less than $4 \%$, which is comparable to shrinkage found in literature. ${ }^{[27]}$ The extent of shrinkage depends on factors such as curing temperature, curing time and layer thickness. ${ }^{[28]}$ Shrinkage is the result of the change in volume upon crosslinking of the PDMS precursor and upon cooling of the structure from curing temperature to room temperature. While shrinkage will be higher at higher curing temperatures, an increased temperature is desirable as curing rates significantly increase and device manufacturing times could be minimized.
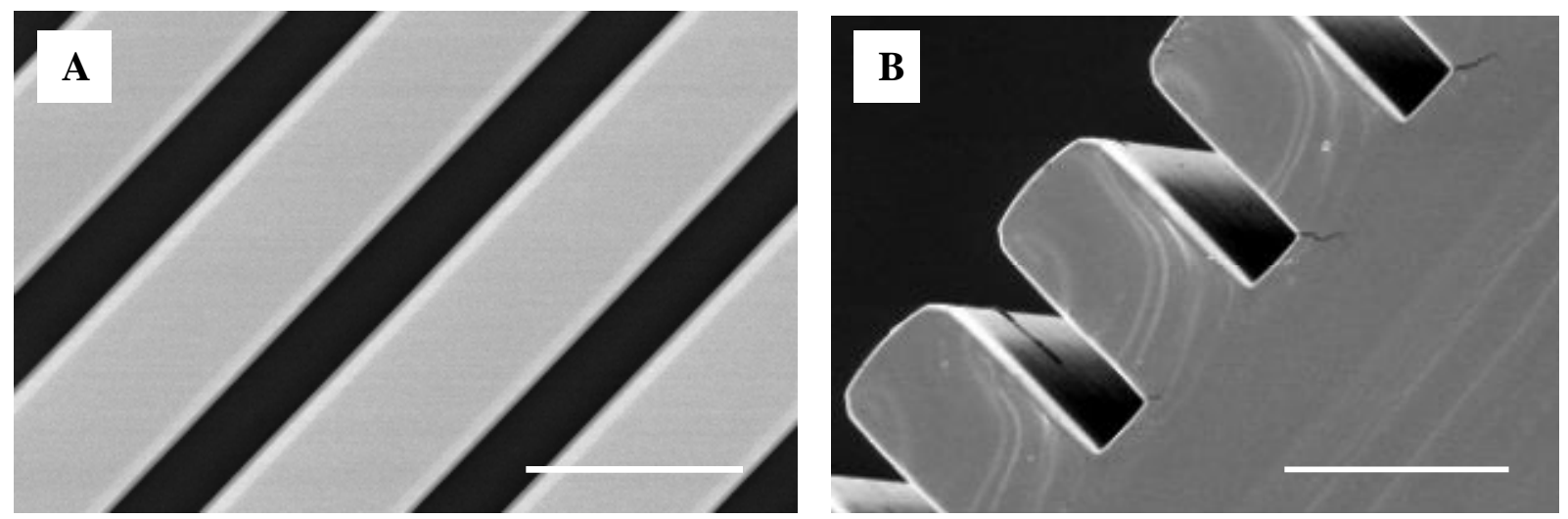

Figure 2. PDMS replica obtained by casting and thermal curing on silicon master A. Top view (A). Cross-section (B). Note the cracks that have formed (Scale bars are 50 $\mu \mathrm{m}$ ). 
Table 1. Dimensions of the microstructures on the surface of PDMS replicas obtained by casting and thermal curing on silicon masters A and B (see also Figure 1). Data are presented as mean values \pm standard deviation of five measurements.

\begin{tabular}{|c|c|c|c|c|c|}
\hline \multirow{2}{*}{ replica } & \multirow{2}{*}{ master } & $\mathbf{a}^{\mathrm{a}}$ & $\mathbf{b}^{\mathrm{a}}$ & $\mathbf{c}^{\mathrm{a}}$ & shrinkage \\
\hline & & $\mu \mathbf{m}$ & $\mu \mathbf{m}$ & $\mu \mathbf{m}$ & $\%$ \\
\hline \multirow{2}{*}{ PDMS } & A & $17.1 \pm 0.3$ & $32.3 \pm 0.1$ & $54.7 \pm 0.4$ & $3.8 \pm 0.5$ \\
\hline & B & $309.7 \pm 1.4$ & $48.8 \pm 0.9$ & $53.3 \pm 1.2$ & $2.2 \pm 0.3$ \\
\hline
\end{tabular}

\footnotetext{
${ }^{a}$ In the microstructured replicas, the dimensions a correspond to the width of the channels, the dimensions $\mathbf{b}$ to the width of the ridges and the dimension $\mathbf{c}$ to the depth of the channels. See Figure 1.

${ }^{\mathrm{b}}$ The shrinkage was determined by comparing the sums of the widths of the channels and ridges of the microstructured replicas with those of the masters.
}

In finding a compromise between avoiding extensive shrinking by curing at low temperatures and curing at elevated temperatures to reduce curing times, PDMS is usually cured at $80{ }^{\circ} \mathrm{C}$ for approximately 2 hours. ${ }^{[23]}$ It should be noted that in industrial manufacturing and high throughput fabrication these long times might be a limiting factor.

\section{Replication of Microstructures on PTMC Surfaces}

Three-armed PTMC macromers with average molecular weights $\left(\overline{\mathrm{M}}_{n}\right)$ between 3500 and $41000 \mathrm{~g} / \mathrm{mol}$ were synthesized by ring-opening polymerization and subsequent functionalization with methacryloyl chloride or methacrylic anhydride. ${ }^{1} \mathrm{H}-\mathrm{NMR}$ spectroscopy confirmed conversions of more than $97 \%$ and degrees of functionalization with photo-crosslinkable end-groups between 71 and $88 \%$. The microstructures of both silicon masters, depicted in Figure 1, could readily be replicated into PTMC surfaces by casting or hot embossing, and subsequent network formation by photo-crosslinking. Figure 3 gives an overview of several microstructured PTMC surfaces obtained by replication of silicon master A using different PTMC resin formulations.

Similar to the uncured PDMS base material, the PTMC 3500 resin is a viscous liquid at room temperature and can be cast directly onto the microstructured silicon wafer. However, by illuminating the resin for only a few minutes, a cured photo-crosslinked PTMC material with a microstructured surface is obtained. The microstructures of silicon master A can be faithfully reproduced in the PTMC 3500 material (see Figure 3A and 3B). 

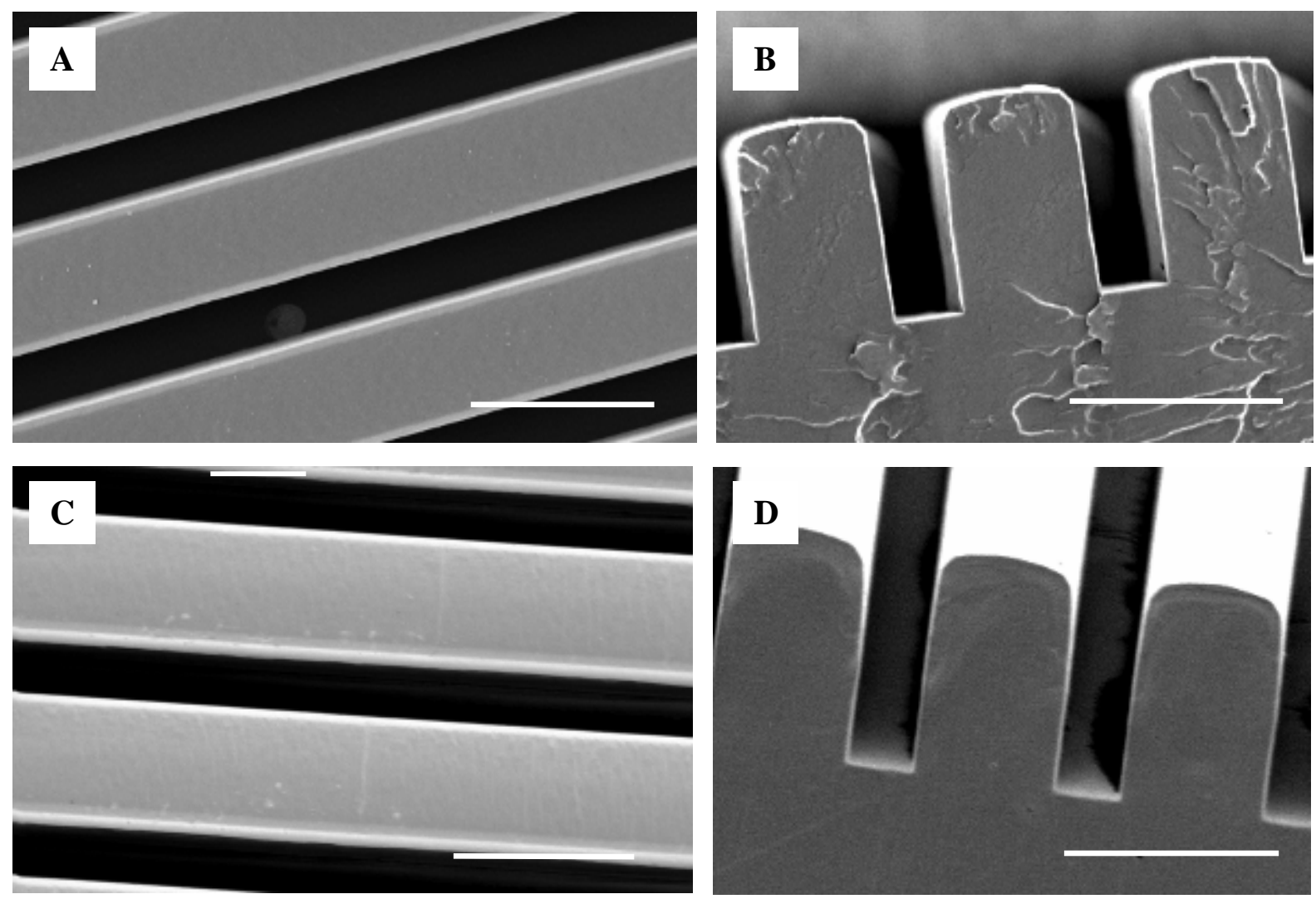

Figure 3. Scanning electron microscopy images of microstructured PTMC materials obtained using resins based on PTMC macromers of different molecular weight. Specimens were prepared by casting and subsequent photo-crosslinking of a PTMC 3500 resin $(A, B)$ or by hot embossing followed by photo-crosslinking of a resin based on PTMC 25400 (C) or PTMC 41000 (D). A microstructured silicon wafer (master A in Figure 1) was employed as template. (Scale bars are $50 \mu \mathrm{m})$.

The corresponding dimensions of the channels and ridges, as determined from the SEM images, are specified in Table 2. Although the features of the master are accurately reproduced, shrinkage is relatively high. The determined value of $7.5 \%$ shrinkage is approximately twice that of PDMS. The shrinkage is likely due to the relatively high crosslink density of the formed network obtained after photo-crosslinking PTMC macromers with relatively low molecular weights of $3500 \mathrm{~g} / \mathrm{mol}$. Although shrinkage can be taken into account when designing a template, it is undesired. 
Table 2. Dimensions of the microstructures on the surface of PTMC replicas obtained by casting or hot embossing and subsequent photo-crosslinking on silicon masters $A$ and $B$ (see also Figure 1). Data are presented as mean values \pm standard deviation of five measurements.

\begin{tabular}{|c|c|c|c|c|c|}
\hline \multirow{2}{*}{ replica } & \multirow{2}{*}{ master } & \multirow{2}{*}{$\frac{\mathbf{a}^{\mathrm{a}}}{\mu \mathbf{m}}$} & \multirow{2}{*}{$\frac{\mathbf{b}^{\mathrm{a}}}{\mu \mathbf{m}}$} & \multirow{2}{*}{$\frac{c^{a}}{\mu m}$} & \multirow{2}{*}{$\frac{\text { shrinkage }}{\%}$} \\
\hline & & & & & \\
\hline $\begin{array}{l}\text { PTMC } 3500 \text {, } \\
\text { casting }\end{array}$ & A & $15.7 \pm 0.4$ & $31.9 \pm 0.4$ & $49.0 \pm 0.5$ & $7.5 \pm 0.3$ \\
\hline PTMC 25400, & A & $16.2 \pm 0.3$ & $32.3 \pm 0.3$ & $49.3 \pm 0.2$ & $5.7 \pm 1.1$ \\
\hline hot embossing & $\mathrm{B}$ & $309.2 \pm 2.5$ & $50.7 \pm 1.5$ & $50.6 \pm 0.2$ & $1.7 \pm 0.4$ \\
\hline $\begin{array}{l}\text { PTMC 41000, } \\
\text { hot embossing }\end{array}$ & A & $15.9 \pm 0.6$ & $33.5 \pm 0.7$ & $53.5 \pm 0.4$ & $3.1 \pm 1.7$ \\
\hline
\end{tabular}

${ }^{a}$ In the microstructured replicas, the dimensions a correspond to the width of the channels, the dimensions $\mathbf{b}$ to the width of the ridges and the dimension $\mathbf{c}$ to the depth of the channels. See Figure 1.

${ }^{\mathrm{b}}$ The shrinkage was determined by comparing the sums of the widths of the channels and ridges of the microstructured replicas with those of the silicon templates.

A lower network crosslink density can be obtained when using PTMC macromers of higher molecular weight, which may not only reduce shrinkage, but also could improve the mechanical properties of the formed networks, especially the resistance to tearing. ${ }^{[19]}$ Table 3 lists the mechanical properties of PTMC networks obtained from macromer molecular weights similar to the ones used in this work. With increasing macromer molecular weight the volume degree of swelling increased, which indicates a decrease in the crosslink density of networks. The network obtained from PTMC 3300 is highly crosslinked, resulting in a slightly higher E-Modulus than networks based on macromer molecular weights above $10000 \mathrm{~g} / \mathrm{mol}^{\left[{ }^{[19]}\right.}$ However, the increase in macromer molecular weight strongly affects the tensile strength, elongation at break and the tear strength, resulting in tough, elastic, and flexible networks. When comparing these results with values obtained for PDMS (also given in Table 3), it can be seen that PTMC networks based on higher macromer molecular weights exceed the mechanical properties of PDMS, especially when it comes to tear resistance. This tear resistance is important when microstructures are used for example in microfluidic devices, where inlets and outlets have to be inserted in the material. 
Table 3. Mechanical Properties of PTMC networks based on macromers of different molecular weights and PDMS. (Taken from reference 19).

\begin{tabular}{|c|c|c|c|c|c|}
\hline material & $\begin{array}{c}\text { volume } \\
\text { degree of }\end{array}$ & E-Modulus & $\begin{array}{c}\text { tensile } \\
\text { strength }\end{array}$ & $\begin{array}{l}\text { elongation } \\
\text { at break }\end{array}$ & $\begin{array}{c}\text { tear } \\
\text { strength }\end{array}$ \\
\hline & swelling & MPa & $\mathbf{N} / \mathbf{m m}^{2}$ & $\%$ & $\mathbf{N} / \mathbf{m m}$ \\
\hline PTMC 3300 & $4.7 \pm 0.2$ & $7.5 \pm 0.2$ & $3.5 \pm 0.4$ & $78 \pm 6$ & $1.61 \pm 0.02$ \\
\hline PTMC 24600 & $12.3 \pm 0.4$ & $5.2 \pm 0.2$ & $9.8 \pm 1.4$ & $822 \pm 29$ & $4.88 \pm 0.93$ \\
\hline PTMC 41000 & $16.9 \pm 0.8$ & $4.9 \pm 0.5$ & $17.3 \pm 3.3$ & $1227 \pm 94$ & $11.09 \pm 1.51$ \\
\hline PDMS & n.d. & $2.6 \pm 0.2$ & $8.7 \pm 0.2$ & $164 \pm 15$ & $0.19 \pm 0.01$ \\
\hline
\end{tabular}

n.d. $=$ not determined

Methacrylate-functionalized PTMC macromers with molecular weights more than approximately $10000 \mathrm{~g} / \mathrm{mol}$ are solids at room temperature. ${ }^{[19]}$ One way to allow them to be cast onto the microstructured silicon wafers is by dissolving the macromers in a diluent. In preliminary experiments we dissolved PTMC 25400 in a non-reactive diluent. After addition of an initiator, the solutions were cast on the silicon templates and photo-cured. A crosslinked gel was obtained, which after extraction with a non-solvent and drying showed a microstructured surface. However, the features were irregular and did not accurately replicate the microstructures of the masters (data not shown).

Much better quality microstructured PTMC surfaces are obtained when the use of diluents is avoided. For the PTMC macromers with high molecular weights that are solid at room temperature, hot embossing can be used. Resin compositions based on PTMC 25400 and PTMC 41000 macromers were hot embossed at $120{ }^{\circ} \mathrm{C}$ for 2 min using the microstructured silicon wafers, and subsequently photo-crosslinked for $5 \mathrm{~min}$. Images of these replicates are shown in Figure 3C and 3D. As shown by the data in Table 2, the microstructures of the master were accurately replicated and of comparable quality and fidelity as the ones obtained using liquid cast PTMC 3500 or PDMS. When using the microstructured silicon master A, the determined shrinkage was $5.7 \%$ for PTMC 25400 structures and $3.1 \%$ for PTMC 41000 structures. These values are close to the ones found for PDMS. A lower shrinkage of only $1.7 \%$ was determined for microstructured PTMC 24500 surfaces hot embossed and photo-cured using master B. 
For these resins, shrinkage of the microstructures is mainly due to the elevated temperature at which the macromers were crosslinked, as well as subsequent cooling to room temperature. By hot embossing and photo-crosslinking at lower temperatures, it might be possible to obtain structured surfaces where shrinkage is even lower. This still remains to be optimized.

Hot embossing combined with photo-crosslinking of PTMC macromers of relatively high molecular weight, allows the very rapid manufacturing of microstructured surfaces. The accuracy and fidelity of replication is quite comparable to PDMS. Therefore, this method is not only cost-efficient, but also suitable for high-throughput processing and manufacturing. ${ }^{[29]}$

As discussed previously, the photo-crosslinked PTMC 25400 and PTMC 41000 networks described in this work are much tougher and more resistant to tearing than the PDMS networks. Their E-modulus values are also somewhat higher, which could make them a more suited material for the preparation of nanostructured specimens. This will be investigated in future experiments.

\section{Protein Printing Using a Microstructured PTMC Stamp}

In microcontact printing ( $\mu \mathrm{CP}$ ) stamps are often prepared using PDMS, as it is a flexible and elastic material that (as was also shown above) can readily be microstructured. However, PDMS is also quite hydrophobic with a water contact angle of approximately $113^{\circ}$. This means that in $\mu \mathrm{CP}$ of polar inks, such as solutions of biomolecules in buffers, the surface of the PDMS stamp that is to be used needs to be oxidized. ${ }^{[7,30]}$

Crosslinked PTMC networks, prepared by photo-crosslinking PTMC macromers are much less hydrophobic and have water contact angles varying between 89 and $98^{\circ}{ }^{[31]}$ As it also is elastomeric at room temperature, we investigated the use of photo-crosslinked microstructured PTMC 25400 as stamp to allow transfer of a pattern of biomolecules to a glass surface. The stamp was obtained by hot embossing and photo-crosslinking the resin on master B as described before. As a model protein, bovine serum albumin labelled with fluorescein isothiocyanate (BSA/FITC) dissolved in phosphate buffered saline was used as the ink.

The surface of the PTMC 25400 stamps consisted of parallel channels with a width and a depth of 309 and $51 \mu \mathrm{m}$, respectively. These channels were separated by ridges with a width of $51 \mu \mathrm{m}$ (see Table 2). After inking the stamps and removing the excess BSA/FITC 
solution, the stamp was brought into conformal contact with the glass surface. Stamps were inked for 30 or $60 \mathrm{~min}$, and contacted with the glass surface for times varying between 5 and 30 min. Figure 4 shows confocal laser scanning microscopy images of the patterns of the transferred fluorescently labeled protein.
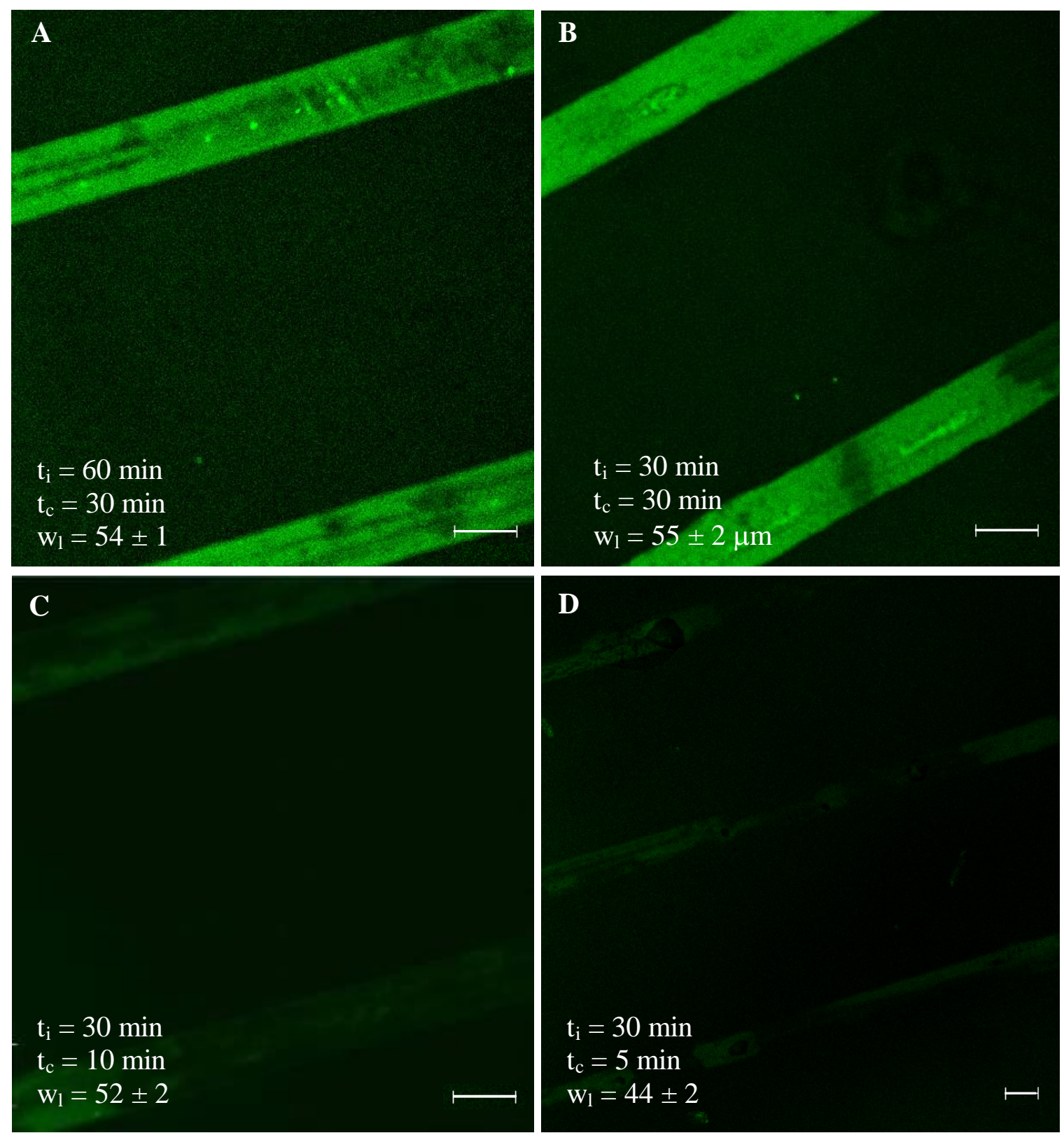

Figure 4. Confocal laser scanning microscopy images obtained after patterning BSA/FITC on a glass surface using a PTMC 25400 stamp. In the microstructured stamp, the width of the ridge was $50.7 \pm 1.5 \mu$ m (master template B, see Figure 1 and Table 2). Different inking times $\left(t_{i}\right)$ and contacting times $\left(t_{c}\right)$ were applied to transfer the pattern. The width of the transferred BAS/FITC pattern $\left(w_{l}\right)$ is given as the average \pm standard deviation of five measurements. (Scale bars are $50 \mu \mathrm{m})$. 
The figure clearly shows that BSA/FITC was successfully transferred to the glass surface when the ridges of the microstructured PTMC stamp are contacted with the surface of the glass. In most cases, the width of the transferred protein pattern coincides well with the ridge width of the microstructured PTMC stamp $(51 \mu \mathrm{m})$. Comparison of Figures 4A and 4B indicate no significant differences in the pattern of the transferred protein between inking times of 30 and $60 \mathrm{~min}$ when the contacting time was $30 \mathrm{~min}$. With inking times of $30 \mathrm{~min}$ and contacting times decreasing from 30 to $5 \mathrm{~min}$, the intensity of the fluorescence of the patterned protein lines noticeably decreased as is shown in Figure 4B, 4C and 4D. At the shortest contacting time of $5 \mathrm{~min}$, also the width of the transferred protein line decreased significantly. These results suggest that more protein is transferred at longer contacting times.

In conclusion, microstructured PTMC can be used as stamps without additional surface modification to effectively transfer a pattern of proteins to a substrate. More extended investigations will be required to reveal the suitability of microstructured PTMC stamps to print other biologically interesting inks.

\section{Cell Culturing on Crosslinked PTMC and PDMS Surfaces}

Previously we showed that human umbilical vein endothelial cells (HUVECs) showed good to excellent adhesion and cell proliferation when cultured on the surface of smooth photo-crosslinked PTMC. ${ }^{[21]}$ In that experiment a PTMC macromer was photo-crosslinked and subsequently extracted to remove non-reacted macromer, initiator residues and/or inhibitors.

Here we investigate the behavior of mesenchymal stem cells (MSCs) on photo-crosslinked PTMC 25400 that was not extracted prior to cell culturing. MSCs are multipotent plastic cells that exhibit trophic activity, differentiating into different cell types and transdifferentiating into diverse lineage phenotypes. They secrete bioactive molecules that provide a regenerative microenvironment for a variety of injured adult tissues, making them valuable mediators for tissue repair and regeneration. MSCs are present in a variety of tissues during human development, and have often been used in preclinical models for tissue engineering. ${ }^{[32-33]}$

Figure 5 shows MSCs cultured on non-structured PTMC surfaces, as well as on microstructured PTMC surfaces prepared by hot embossing and photo-crosslinking using 
both silicon wafer templates A and B (see Figure 1, Figure 3 and Table 2). Surfaces measuring approximately $7 \times 10 \mathrm{~mm}^{2}$ were seeded with $3 \times 10^{4}$ cells $/ 100 \mu 1$ of medium.
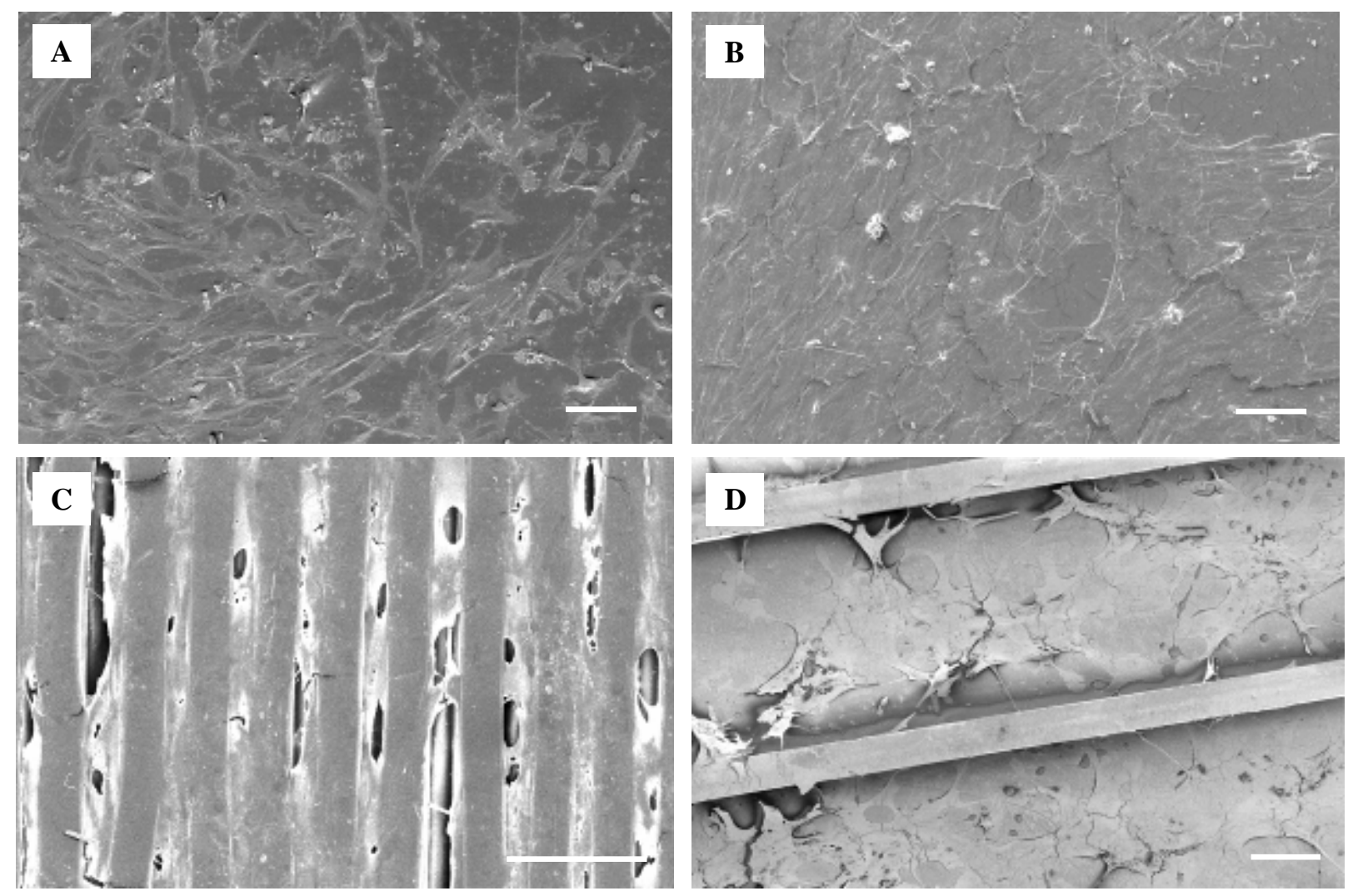

Figure 5. Scanning electron microscopy (images of human MSCs cultured on photocrosslinked PTMC 25400 surfaces. Adhesion of cells on smooth PTMC surfaces after 1 day (A) and after 3 days (B), and after 1 day on on microstructured PTMC surfaces obtained by replication of silicon wafer template $A(C)$ and template $B(D)$. (Scale bars are $100 \mu \mathrm{m})$.

Figure 5A and 5B show the adhering MSCs on the surface of smooth PTMC 25400 after culturing for 1 and 3 days, respectively. The cells are evenly distributed and show a stretched morphology after 1 day, and proliferate on the surface of the material to reach near confluency after 3 days. Also on the microstructured PTMC 25400 surface (replicated from master A) the cells adhere and grow well. Figure 5C and 5D indicate that at day 1 the cells are homogeneously distributed, showing a stretched morphology and nearly cover the entire channel surface. Although the cell seeding density was relatively high, the cells remained viable for the duration of the experiment (7 days).

On the microstructured PTMC 25400 surfaces with narrow channels the cells mainly adhered to the ridges that separate the channels (Figure 5C). Interestingly, in adhering to 
each other and to the material, the forces exerted by the cells deform the parallel ridges on the surface. When seeded onto microstructured PTMC 25400 with much wider channels that was prepared using master B, the majority of MSC cells adhered to, spread and proliferated on the bottom of the channels. Only few cells cross over the ridges to other channels. This is similar to what has been reported by Papenburg et al.. ${ }^{[34]}$

When culturing human MScs on similar PDMS surfaces under the same conditions, it was observed that after 1 day of culture the cells had already reached near confluency. However, the interaction between the cells and PDMS was very weak and the cells detached from both the microstructured and the non-structured surfaces when processing for SEM (data not shown).

As the cell seeding density used was relatively high, it is not possible to differentiate between crosslinked PTMC and PDMS surfaces with regard to differences in human MSC cell proliferation behavior. Nevertheless, the adhesion of the cells to PTMC surfaces is stronger than to PDMS. Therefore we can state that, even without extracting the networks, photo-crosslinked PTMC is well compatible with MSCs. Photo-crosslinked PTMC could be a welcome alternative to PDMS in biological applications and in tissue engineering.

\section{Conclusions}

Microstructured poly(trimethylene carbonate) surfaces can easily and rapidly be prepared by hot embossing and subsequently photo-crosslinking resins based on PTMC macromers. Within a few minutes, the surface of a microstructured silicon wafer is accurately replicated. The fidelity of the replicated structures is comparable to those obtained using poly(dimethylsiloxane), but the process is significantly faster taking only minutes to complete. This allows preparation of these microstructured materials in high-throughput processes. We explored two possible applications of microstructured PTMC that are of interest in the biomedical field.

In a first set of experiments, we explored the feasibility of using these microstructured PTMC to pattern substrates with proteins as an alternative for PDMS stamps. PTMC stamps, cut from microstructured PTMC which was prepared from a resin based on the macromer molecular weights of $25400 \mathrm{~g} / \mathrm{mol}$, was successfully applied as a stamp to pattern a glass surface with fluorescently labeled bovine serum albumin as a model protein at high resolutions. 
In a second set of experiments, we investigated the use of microstructured PTMC as substrate material for culturing human mesenchymal stem cells. Already after one day of cell culturing, MSCs strongly adhered to the photo-crosslinked PTMC surfaces. After 3 days the cells showed near confluency and remained viable for at least 7 days. It is noteworthy to mention that these PTMC networks were not extracted or purified in another manner. Cells cultured on PDMS surfaces, on the other hand, adhered only weakly to the PDMS substrates and easily detached when processed for SEM after 1 day of culturing.

The fact that photo-crosslinked PTMC networks are degradable in biological environments makes them useful for use in biodegradable implant devices and tissue engineering applications. Additionally, PTMC networks show mechanical properties which are superior to those of PDMS. The prepared photo-crosslinked PTMC networks are excellent alternative materials to PDMS in preparing microstructured devices by soft lithography for use in the life sciences.

\section{References}

[1] Voldman J, Gray ML, Schmidt MA. Microfabrication in biology and medicine. Annu Rev Biomed Eng 1999, 1, 401-425.

[2] Breslauer DN, Lee PJ, Lee LP. Microfluidics-based systems biology. Mol Biosyst 2006, 2, 97-112.

[3] Xia Y, Whitesides GM. Soft Lithography. Angew Chem Int Ed 1998, 37, 550-575.

[4] Weibel DB, DiLuzio WR, Whiteside GM. Microfabrication meets microbiology. Nat Rev Microbiol 2007, 5, 209-218.

[5] Kumar A, Whitesides GM. Features of gold having micrometer to centimeter dimensions can be formed through a combination of stamping with an elastomeric stamp and an alkanethiol "ink" followed by chemical etching. Appl Phys Lett 1993, 63, 2002-2004.

[6] Delamarche E. Microcontact printing of proteins. Nanobiotechnol 2004, 31-52.

[7] Kaufmann T, Ravoo BJ. Stamps, inks and substrates: polymers in microcontact printing. Polym Chem 2010, 1, 371-387.

[8] Perl A, Reinhoudt DN, Huskens J. Microcontact printing: limitations and achievements. Adv Mater 2009, 21, 2257-2268.

[9] Xu H, Huskens J. Versatile stamps in microcontact printing: transferring inks by molecular recognition and from ink reservoirs. Chem Eur J 2010, 16, 2342-2348.

[10] Whitesides GM. The origins and the future of microfluidics. Nature 2006, 442, 368373.

[11] McDonald JC, Whitesides GM. Poly(dimethylsiloxane) as a material for fabricating microfluidic devices. Accounts Chem Res 2002, 35, 491-499.

[12] Andersson H, van den Berg A. Microfabrication and microfluidics for tissue engineering: state of the art and future opportunities. Lab Chip 2004, 4, 98-103. 
[13] Leclerc E, Sakai Y, Fujii T. Cell culture in 3-dimensional microfluidic structure of PDMS (polydimethylsiloxane). Biomed Microdevices 2003, 5, 109-114.

[14] Borenstein JT, Terai H, King KR, Weinberg EJ, Kaazempur-Mofrad MR, Vacanti JP. Microfabrication technology for vascularized tissue engineering. Biomed Microdevices 2002, 4, 167-175.

[15] Sarkar S, Dadhania M, Rourke P, Desai TA, Wong JY. Vascular tissue engineering: microtextured scaffold templates to control organization of vascular smooth muscle cells and extracellular matrix. Acta Biomater 2005, 1, 93-100.

[16] Jo B-H, Van Lerberghe LM, Motsegood KM, Beebe DJ. Three-dimensional microchannel fabrication in polydimethylsiloxane (PDMS) elastomer. J Microelectromech $S$ 2000, 9, 76-81.

[17] Bettinger EJ, Weinberg EJ, Kulig KM, Vacanti JP, Wang Y, Borenstein JT, Langer R. Three-dimensional microfluidic tissue engineering scaffolds using a flexible biodegradable polymer. Adv Mater 2006, 18, 165-169.

[18] Moraes C, Kagoma YK, Beca BM, Tonelli-Zasarsky RLM, Sun Y, Simmons CA. Integrating polyurethane culture substrates into poly(dimethylsiloxane) microdevices. Biomaterials 2009, 30, 5241-5250.

[19] Chapter 3 of this thesis.

[20] Chapter 6 of this thesis.

[21] Chapter 7 of this thesis.

[22] Chapanian R, Tse MY, Pang SC, Amsden BG. The role of oxidation and enzymatic hydrolysis on the in vivo degradation of trimethylene carbonate based photocrosslinkable elastomers. Biomaterials 2009, 30, 295-306.

[23] Liu M, Sun J, Chen Q. Influences of heating temperature on mechanical properties of polydimethylsiloxane. Sensor Actuat A-Phys 2009, 151, 42-45.

[24] Dow Corning Product Information, "Information about Dow Corning® brand Silicone Encapsulants," 2005.

[25] Bhagat AAS, Jothimuthu P, Papautsky. Photodefinable polydimethylsiloxane (PDMS) for rapid lab-on-a-chip prototyping. Lab Chip 2007, 7, 1192-1197.

[26] Teixeira S, Fernandes H, Leusink A, van Blitterswijk C, Ferraz MP, Monteiro FJ, de Boer J. In vivo evaluation of highly macroporous ceramic scaffolds for bone tissue engineering. J Biomed Mater Res A 2010, 93A, 567-575.

[27] Wu H, Odom TW, Chiu DT, Whitesides GM. Fabrication of complex threedimensional microchannel systems in PDMS. J Am Chem Soc 2003, 125, 554-559.

[28] Lee SW, Lee SS. Shrinkage ratio of PDMS and its alignment method for the wafer level process. Microsyst Technol 2008, 14, 205-208.

[29] Xia Y, Whitesides GM. Soft lithography. Annu Rev Mater Sci 1998, 28, 153-184.

[30] Mata A, Fleischman AJ, Roy S. Characterization of polydimethylsiloxane (PDMS) properties for biomedical micro/nanosystems. Biomed Microdevices 2005, 7, 281-293.

[31] Chapter 5 of this thesis.

[32] Caplan AI, Bruder SP. Mesenchymal stem cells: building blocks for molecular medicine in the 21st century. Trends Mol Med 2001, 7, 259-264.

[33] Caplan AI. Adult mesenchymal stem cells for tissue engineering versus regenerative medicine. J Cell Physiol 2007, 213, 341-347.

[34] Papenburg BJ, Dooms Rodrigues E, Wessling M, Stamatialis D. Insights into the role of material surface topography and wettability on cell-material interactions. Soft Matter 2010, 6, 4377-4388. 


\title{
Chapter 5
}

\section{Preparation of Flexible and Elastic Poly(Trimethylene Carbonate) Structures by Stereolithography}

\begin{abstract}
Three-dimensional porous and non-porous structures were designed and prepared by stereolithography using resins based on poly(trimethylene carbonate) macromers. Three-armed poly(trimethylene carbonate) macromers with molecular weights varying between 3250 and $27750 \mathrm{~g} / \mathrm{mol}$ were synthesized. Photo-crosslinkable resins containing the PTMC macromers, propylene carbonate as non-reactive diluent, a photo-initiator and a dye were formulated and processed by stereolithography at temperatures between room temperature and $65{ }^{\circ} \mathrm{C}$.

Tough, flexible network films prepared in this manner had $E$ modulus values of approximately 3.8 MPa and high elongations at break of more than $900 \%$ after extraction and drying. Maximal tensile strengths were approximately 4.2 MPa. It was found that these values increased with increasing PTMC macromer molecular weight used in their preparation.

Designed solid cylindrical specimens and porous structures with a gyroid pore network architecture could successfully be built at $65^{\circ} \mathrm{C}$ by stereolithography using resins based on PTMC macromers with molecular weights up to $20150 \mathrm{~g} / \mathrm{mol}$. To reach suitable viscosities for processing, these resins necessarily contained up to $45 \mathrm{wt} \%$ propylene carbonate as nonreactive diluent.

In compression testing the solid specimens had compression moduli of 3.1 to $4.2 \mathrm{MPa}$, similar to the values determined in tensile testing. The porous structures had porosities of 53 to $66 \%$ and average pore sizes of 309 to $407 \mu \mathrm{m}$. The compression moduli of the porous structures were significantly lower than those of the solid structures with values ranging from $20 \mathrm{kPa}$ for structures with the highest porosity to $156 \mathrm{kPa}$ for structures with the lowest porosity.
\end{abstract}




\section{Introduction}

Stereolithography allows the fabrication of decimeter sized three-dimensional structures at high resolutions. ${ }^{[1]}$ With stereolithography, which is the most versatile, accurate and precise technique among other rapid prototyping techniques, (computer-) designed constructs can be built in a layer-by-layer manner. ${ }^{[2]}$ A volume element of a liquid, photo-crosslinkable resin is solidified upon illumination with (laser) light. Software slices a three-dimensional computer design into sequential layers of masks. Each mask consists of an individual two dimensional pixel pattern, which defines the position of the liquid resin voxels that are to be illuminated to induce solidification. Upon computer-controlled sequential photocrosslinking of the layers through the masks, a three-dimensional structure is created when each new layer is cured on top of the preceding layer by careful control of the light penetration depth. ${ }^{[3]}$

Stereolithography can be used in this manner to reproducibly prepare three-dimensional tissue engineering scaffolds with well-defined pore network architectures. Structures with defined porosities, pore sizes and pore size distributions, pore network architectures, pore interconnectivities and even gradients in porosity and pore size can be designed and built at high resolution. ${ }^{[4]}$ However, for applications in medicine the number of biocompatible resins suitable for stereolithography is still very limited, especially when resorbable implants or scaffolds such as in tissue engineering are required. For these purposes, resins based on poly(propylene fumarate), poly(ethylene glycol), poly(D,L-lactide) and (co)polymers of trimethylene carbonate and $\varepsilon$-caprolactone have been investigated. These resins result in the formation of brittle or rigid networks. ${ }^{[3,5-12]}$ For use in stereolithography, only few resins have been developed that yield flexible and elastic networks for soft tissue engineering purposes. These resins were based on functionalized poly(D,L-lactide-co- $\varepsilon$-caprolactone) or poly(trimethylene carbonate) (PTMC) oligomers of low molecular weight. ${ }^{[4,13]}$

We showed in previous work that the mechanical properties of networks prepared by photocrosslinking PTMC macromers in the melt were strongly dependent on the molecular weight of the macromer from which they were prepared. ${ }^{[14]}$ While the flexibility of the networks did not significantly change with increasing PTMC macromer molecular weights, E modulus values were in the range of 4 to $8 \mathrm{MPa}$, we found that for networks prepared from PTMC macromers with molecular weights higher than $10000 \mathrm{~g} / \mathrm{mol}$ the tensile 
strength, toughness, tear resistance and suture retention strength significantly increased with increasing macromer molecular weight.

It would be most beneficial to be able to prepare designed structures by stereolithography using resins based on PTMC macromers with molecular weights higher than $10000 \mathrm{~g} / \mathrm{mol}$. The aim of this work is to investigate the applicability of resins based on PTMC macromers with these high molecular weights in stereolithography. Methacrylate end-functionalized PTMC macromers of different molecular weights were synthesized and diluted with propylene carbonate to decrease the resin viscosity. The processability of these resins in stereolithography after optimization of diluent concentration, photo-crosslinking temperature and irradiation times was assessed. From these resins designed porous and nonporous structures were built and characterized with respect to their morphological, physical and mechanical properties.

\section{Materials and Methods}

\section{Materials}

Polymer grade 1,3-trimethylene carbonate (1,3-dioxan-2-one, TMC) was obtained from Boehringer Ingelheim (Germany). Glycerol (99.5+\% spectrophotometric grade) was purchased from Aldrich (USA) and dehydrated by briefly heating at $180{ }^{\circ} \mathrm{C}$. Stannous octoate (stannous 2-ethylhexanoate, SnOct $_{2}$ ) was used as received from Sigma (USA). Methacrylic anhydride (94\%) and hydroquinone were purchased from Aldrich (Germany), and ( \pm )- $\alpha$-Tocopherol (vitamin E) from Fluka (Switzerland). Triethyl amine (used as received) and methacryloyl chloride (distilled prior to use) were obtained from Fluka (Germany). Lucirin TPO-L (ethyl 2,4,6-trimethylbenzoylphenylphosphinate) was kindly provided by BASF (Germany). Orasol Orange G was obtained from Ciba Specialty Chemicals (Switzerland). Serva silicone solution was purchased from SERVA Electrophoresis GmbH (Germany). Technical grade ethanol was obtained from Chempopack (Belgium), and analytical grade dichloromethane from Biosolve (The Netherlands) which was dried over calcium hydride and freshly distilled before use. Propylene carbonate was purchased from Merck (Germany). 


\section{Macromer Synthesis and Resin Formulation}

Three-armed PTMC macromers were prepared by a two-step synthesis described in

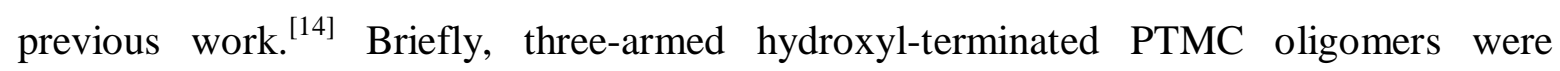
prepared by ring opening polymerization (oligomerization) of TMC on a 100-130 g scale using glycerol as initiator and $\operatorname{SnOct}_{2}(0.07 \mathrm{wt} \%)$ as catalyst. The molecular weights of the oligomers could be controlled by adjusting the TMC to glycerol ratio. These oligomers were then functionalized by reaction with an excess of methacrylic anhydride. Functionalization of the oligomer with the highest molecular weight was most effectively done using methacryloyl chloride. The monomer conversions, molecular weights (number average molecular weights, $\overline{\mathrm{M}}_{n}$ ) and degrees of functionalization were determined by ${ }^{1} \mathrm{H}-\mathrm{NMR}$ (Varian Innova $300 \mathrm{MHz}$, USA) using $\mathrm{CDCl}_{3}$ (Merck, Germany).

PTMC resin formulations were prepared by mixing the macromers with varying amounts of propylene carbonate, and adding $5 \mathrm{wt} \%$ of Lucirin TPO-L photo-initiator and $0.15 \mathrm{wt} \%$ Orasol Orange $\mathrm{G}$ dye when required. The weight percentages are relative to the macromer. The viscosities of the resins were determined at temperatures between 20 and $75{ }^{\circ} \mathrm{C}$ with a Brookfield DV-E rotating spindle viscometer. The apparatus was equipped with a small sample adapter and Brookfield s21 spindle that rotated at 0.3 and $1.0 \mathrm{rpm}$ (shear rates of 0.28 and $0.931 / \mathrm{s}$, respectively).

\section{Stereolithography}

Flat films and three-dimensional structures with a designed architecture were built from the PTMC based resins by stereolithography using an EnvisionTec Perfactory Mini Multilens (Germany) stereolithography apparatus (SLA). The wavelength of the blue light ranges between 400 and $550 \mathrm{~nm}$, and has a maximum at approximately $440 \mathrm{~nm}$. Details of the experimental setup (top-down) are given in reference 1. With use of an electrical heating element, the resin could be heated to temperatures of up to $70{ }^{\circ} \mathrm{C}$.

Flat films were prepared by illuminating a rectangular area of the resin of $75 \times 60 \mathrm{~mm}^{2}$ for $5 \mathrm{~min}$ at an intensity of approximately $10 \mathrm{~mW} / \mathrm{cm}^{2}$. The films were subsequently post-cured by irradiation for $30 \mathrm{~min}$ in a crosslinking cabinet (Ultralum, USA, operating at $365 \mathrm{~nm}$ and an intensity of 3-4 $\mathrm{mW} / \mathrm{cm}^{2}$ ) in a nitrogen atmosphere. The resulting films were extracted with a 1:1 mixture of propylene carbonate and ethanol, which was refreshed twice, then washed with ethanol and dried under vacuum until constant weight. To obtain films with a 
thickness of approximately $0.5 \mathrm{~mm}$, differences in shrinkage due to the varying amounts of propylene carbonate diluent in the resin were accounted for.

Cylindrical three-dimensional porous and non-porous structures were designed using K3dSurf v0.6.2 (freeware from http://k3dsurf.sourceforge.net) and Rhinoceros 3D (McNeel Europe) computer software. The designs were adjusted to the diluent concentration in the different resins, to give cylinders with a diameter and height of respectively 4.8 and $6.3 \mathrm{~mm}$ after building by stereolithography, extraction and drying. The porous cylindrical structures were designed to have a gyroid pore network architecture, with an average pore size of $442 \mu \mathrm{m}$ and a porosity of $71 \%$.

The three-dimensional structures were built by stereolithography at a pixel resolution of $16 \times 16 \mu \mathrm{m}^{2}$ and layer thicknesses of $25 \mu \mathrm{m}$. Subsequent layers were illuminated at an intensity of approximately $17 \mathrm{~mW} / \mathrm{cm}^{2}$. The required illumination times depended on the resin composition, and were found to vary between 8 and $16 \mathrm{~s}$ per layer for the solid cylinders and between 11 and $40 \mathrm{~s}$ per layer for the porous structures. To be able to precisely control the penetration depth of the light when preparing the designed structures, small amounts of Orasol Orange dye were added to the resins.

The structures were then carefully extracted. To avoid rupture of the built structures during the extraction process, a 3:1 mixture of propylene carbonate and ethanol was used and refreshed daily for one week. Then small additions of ethanol allowed the structures to slowly shrink to their final dimensions in the course of several days. Finally, the scaffolds were washed with ethanol and dried under vacuum until constant weight.

\section{Characterization of Built PTMC Network Structures}

The network characteristics and the physical properties of the PTMC networks prepared by stereolithography were assessed using the flat film specimens after extraction and drying. The volume degree of swelling (q) was assessed at room temperature by swelling diskshaped specimens ( $8 \mathrm{~mm}$ in diameter, punched out from the extracted films) in chloroform for 2 days. The values were determined according to equation 1 :

$$
q=1+\left(\frac{m_{\text {swollen }}-m_{d r y}}{m_{d r y}}\right) \times \frac{\rho_{P}}{\rho_{S}}
$$


Where $m_{\text {swollen }}$ is the mass of the swollen network, $m_{d r y}$ the mass of the initial dry specimen and $\rho_{S}$ and $\rho_{P}$ are the densities of chloroform $\left(1.48 \mathrm{~g} / \mathrm{cm}^{3}\right)$ and PTMC $\left(1.31 \mathrm{~g} / \mathrm{cm}^{3}\right)$, respectively.

The thermal properties of the PTMC networks were determined by differential scanning calorimetry (DSC) using a Perkin Elmer Pyris 1 (USA) calibrated with cyclohexane, indium and lead standards. Samples of 5-12 mg were heated from $-100{ }^{\circ} \mathrm{C}$ to $100{ }^{\circ} \mathrm{C}$ at a heating rate of $10{ }^{\circ} \mathrm{C} / \mathrm{min}$ and quenched rapidly $\left(300{ }^{\circ} \mathrm{C} / \mathrm{min}\right)$ to $-100{ }^{\circ} \mathrm{C}$. After $5 \mathrm{~min}$, a second scan was recorded from which the glass transition temperatures were determined. Onset values $\left(\mathrm{T}_{\mathrm{g}}\right.$, (onset)) and midpoint values of the heat capacity change $\left(\mathrm{T}_{\mathrm{g}}\right.$, (midpoint)) were determined.

The mechanical properties of the PTMC network films were determined at room temperature using a Zwick Z020 tensile tester (Germany), equipped with a $500 \mathrm{~N}$ load cell according to ASTM D882-91 at a cross-head speed of $50 \mathrm{~mm} / \mathrm{min}$ using specimens with lengths of $65.1 \pm 3.0 \mathrm{~mm}$ and widths of $5.4 \pm 0.2 \mathrm{~mm}$. The elongation of the specimens was derived from the grip-to-grip separation, which initially was $50 \mathrm{~mm}$. The yield stress and the elongation at yield were determined from the intersection of tangents to the stress-strain curves. The toughness of the specimens $\left(\mathrm{W}\right.$ in $\mathrm{N} / \mathrm{mm}^{2}$ ) was determined as the areas under the stress-strain curves. Experiments were conducted in triplicate.

To determine their water uptake, disks of $8 \mathrm{~mm}$ in diameter were punched out of the network films and equilibrated in ultra-pure water for $45 \mathrm{~h}$ at room temperature. The water uptake was determined according to:

$$
\text { water uptake }=\left(\frac{m_{w e t}-m_{d r y}}{m_{d r y}}\right) \times 100
$$

where $m_{w e t}$ is the mass of the water-swollen network and $m_{d r y}$ is the mass of the initial dry specimen. Experiments were conducted in triplicate.

Static ultra-pure water (MilliQ Plus, Millipore, France) contact angles of the surfaces of dry PTMC network films were determined using a video-based OCA 20 contact angle system from DataPhysics (Germany), equipped with an electronic syringe module. The measurements were performed at room temperature on five different regions of the film. The presented values are given as average values \pm standard deviation. 
The characteristics of the porous and non-porous designed structures built by stereolithography were evaluated by mechanical testing in compression, microcomputed tomography $(\mu \mathrm{CT})$ and scanning electron microscopy (SEM).

The mechanical behavior of the PTMC structures built by stereolithography using resins based on different PTMC macromer molecular weights was assessed in compression. A Zwick Z020 universal tensile tester was used to compress the specimens at a rate of $30 \% / \mathrm{min}$. Unless mentioned otherwise, the measurements were performed on five extracted and dried structures at room temperature. The presented values are given as average values \pm standard deviation.

Micro-computed tomography ( $\mu \mathrm{CT}$ ) (GE eXplore Locus SP $\mu \mathrm{CT}$ scanner with a spatial resolution of $14 \mu \mathrm{m}$ ) was employed to visualize the porous structures. Porosities and pore size distributions of six different scaffolds were assessed from the $\mu \mathrm{CT}$ data as described elsewhere. ${ }^{[5,15]}$ Average pore sizes were calculated from the pore size distributions. All values are presented as average values \pm standard deviation.

Scanning electron microscopy (SEM) (Philips XL30 ESEM-FEG, The Netherlands) was applied to visualize the porous structures. The apparatus was operated at a voltage of $3 \mathrm{kV}$, and the specimens were sputter coated with gold.

\section{Results and Discussion}

\section{Synthesis of PTMC Macromers and Formulation of Resins}

A series of photo-crosslinkable poly(trimethylene carbonate) macromers of different molecular weight was prepared by ring opening polymerization of trimethylene carbonate in the melt in the presence of varying amounts of glycerol initiator, and subsequent functionalization with methacrylate groups. Monomer conversions were higher than $94 \%$ and the degrees of functionalization varied between 59 and $87 \%$ as determined by ${ }^{1} \mathrm{H}-\mathrm{NMR}$ spectroscopy. The molecular weights of the PTMC macromers were 3250, 10300, 17150, 20150 and $27750 \mathrm{~g} / \mathrm{mol}$, these are respectively named PTMC 3250, PTMC 10300, PTMC 17150, PTMC 20150 and PTMC 27750 throughout the text. With exception of the macromer with the lowest molecular weight, which was a colorless viscous liquid, the obtained PTMC macromers were colorless solids at room temperature. 
It was found in preliminary experiments that the viscosity of the resin should be less than approximately $10 \mathrm{~Pa} \cdot \mathrm{s}$ to adequately build structures by stereolithography. The viscosity of the PTMC macromer resin formulations could be adjusted by addition of propylene carbonate as non-reactive diluent. The amount required to reach a viscosity that is appropriate for our intended purposes strongly depends on the molecular weight of the macromer. ${ }^{[3]}$ While at room temperature a PTMC 3250 resin requires the addition of 30 wt\% propylene carbonate, PTMC macromers of higher molecular weights need more than $50 \mathrm{wt} \%$ diluent to reach viscosities of approximately $10 \mathrm{~Pa} \cdot \mathrm{s}$. Unfortunately, such high diluent concentrations result in extremely fragile (swollen) network structures during the stereolithography building process. The viscosity of the resins can also be decreased by increasing the temperature. At a temperature of $43{ }^{\circ} \mathrm{C}$, PTMC 10300 resins formulated with $32 \mathrm{wt} \%$ propylene carbonate were suited to prepare solid PTMC network films by stereolithography. At $65{ }^{\circ} \mathrm{C}$ an even smaller amount of $22 \mathrm{wt} \%$ of non-reactive diluent was required to be able to build swollen three-dimensional structures.

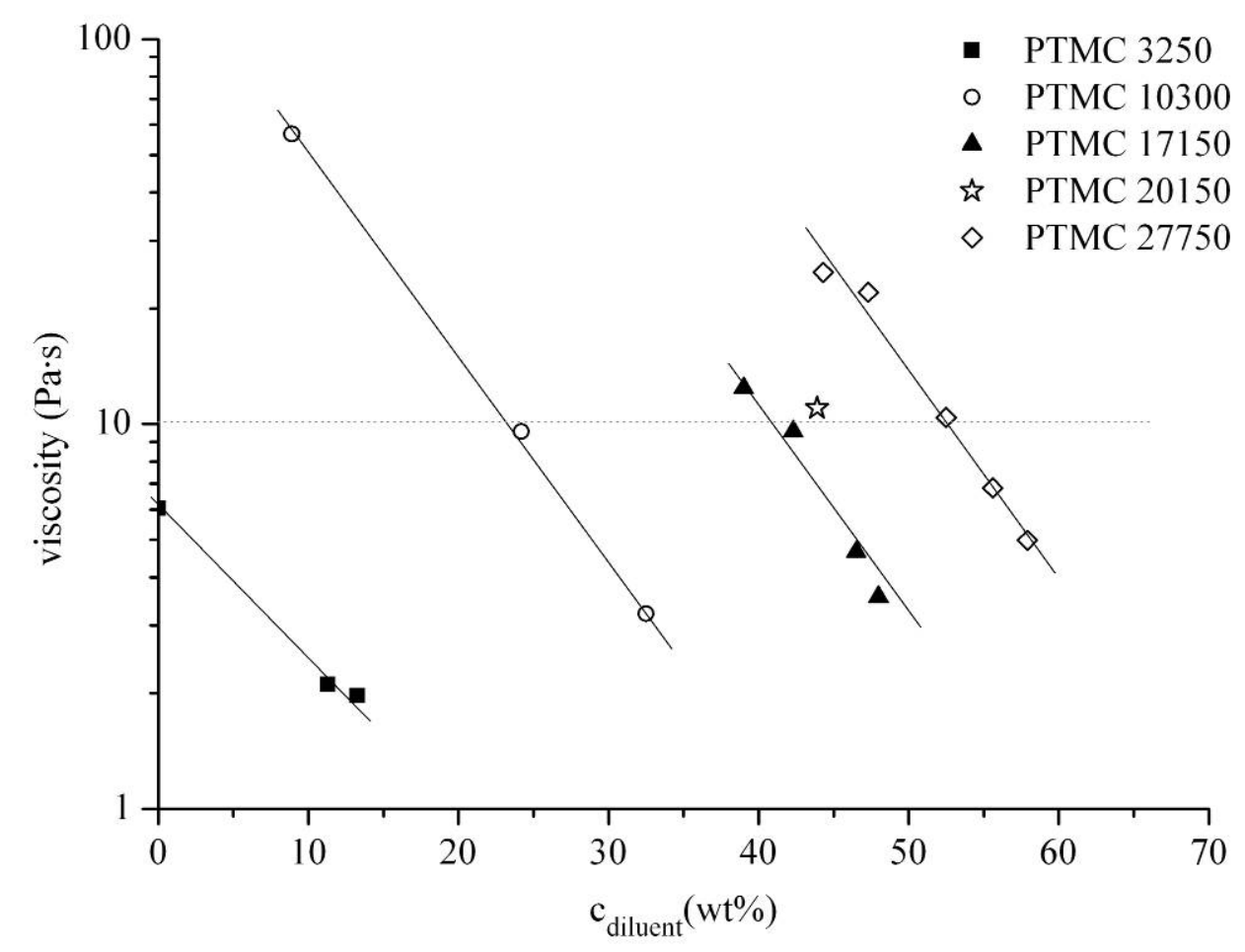

Figure 1. The viscosity of photo-crosslinkable resins based on PTMC macromers of different molecular weights and non-reactive propylene carbonate diluent at $65{ }^{\circ} \mathrm{C}$. A viscosity of approximately $10 \mathrm{~Pa} \cdot \mathrm{s}$ is required for stereolithography. Note the logarithmic scale of the $y$-axis. 
Figure 1 illustrates the change in viscosity of different PTMC macromer resins upon dilution with non-reactive propylene carbonate at $65{ }^{\circ} \mathrm{C}$. It is evident that the viscosity of the resin is strongly dependent on the molecular weight of the macromer and of the amount of diluent added. The least viscous resins are those formulated with the lowest molecular weight PTMC macromers or the highest amounts of diluent. Resins based on PTMC macromers with molecular weights 17150, 20150 and $27750 \mathrm{~g} / \mathrm{mol}$ have an appropriate viscosity for processing by stereolithography when diluted with respectively 40, 45 and $53 \%$ of propylene carbonate. At this temperature, only the PTMC 3250 resin has sufficiently low viscosity to be processed without addition of diluent. (Note that with this macromer structures were built at room temperature using a resin containing $30 \mathrm{wt} \%$ diluent).

\section{PTMC Network Films Prepared by Stereolithography}

To characterize the physical properties of the PTMC networks obtained by stereolithography, resins based on PTMC macromers of different molecular weights were diluted with propylene carbonate to a viscosity of approximately $10 \mathrm{~Pa} \cdot \mathrm{s}$ and photocrosslinked at temperatures between room temperature and $65{ }^{\circ} \mathrm{C}$ to eventually give solid films with a thickness of approximately $0.5 \mathrm{~mm}$. In Table 1 an overview of the applied conditions is given.

Since the photo-crosslinking of the PTMC networks takes place in the presence of solvent, the obtained swollen films were first carefully extracted and dried before assessing their properties.

Table 1 shows that with increasing PTMC macromer molecular weights, the crosslinking density decreases and the degree of swelling of the resulting photo-crosslinked networks increased. When networks are crosslinked in the presence of a non-reactive diluent, their degrees of swelling are significantly higher than when crosslinked in the melt without a diluent. For comparison, a network prepared from a PTMC macromer with molecular weight of $20150 \mathrm{~g} / \mathrm{mol}$ swelled approximately 10 times in chloroform when crosslinked in the melt, but 23 times when crosslinked in the presence of $45 \mathrm{wt} \%$ propylene carbonate. Due to the presence of the non-reactive diluent, the polymer chains are extended (swollen) during the crosslinking process. Networks formed in the diluted state therefore exhibit fewer entanglements and more elastically inactive network chains due to intramolecular reactions, giving super-contracted network chains after deswelling and drying. ${ }^{[16]}$ 
Table 1. PTMC resin formulations and characteristics of extracted and dried crosslinked PTMC network films upon use of the resins in stereolithography.

\begin{tabular}{|c|c|c|c|c|c|}
\hline \multirow{2}{*}{$\begin{array}{l}\text { macromer } \\
\text { used to } \\
\text { prepare } \\
\text { network }\end{array}$} & \multirow{2}{*}{$\frac{\begin{array}{c}\text { diluent } \\
\text { concentration }^{\mathrm{a}}\end{array}}{\mathrm{wt} \%}$} & \multirow{2}{*}{$\frac{\text { temperature }}{{ }^{\circ} \mathrm{C}}$} & \multirow{2}{*}{$\begin{array}{l}\text { degree of } \\
\text { swelling }^{c}\end{array}$} & \multirow{2}{*}{$\frac{\mathbf{T}_{\mathrm{g}} \text { (onset) }}{{ }^{\circ} \mathrm{C}}$} & \multirow{2}{*}{$\frac{\mathbf{T}_{\mathbf{g}} \text { (midpoint) }}{{ }^{\circ} \mathrm{C}}$} \\
\hline & & & & & \\
\hline PTMC 3250 & 30 & 22 & $5.5 \pm 0.2$ & -13.1 & -8.7 \\
\hline PTMC 10300 & 32 & 43 & $11.6 \pm 0.4$ & -16.9 & -13.9 \\
\hline PTMC 17150 & 40 & 65 & $18.6 \pm 0.4$ & -17.1 & -14.7 \\
\hline PTMC 20150 & 45 & 65 & $23.0 \pm 0.4$ & -17.4 & -15.2 \\
\hline PTMC $27750^{\mathrm{d}}$ & 53 & 65 & $43.9 \pm 1.0$ & -19.0 & -17.0 \\
\hline
\end{tabular}

${ }^{\mathrm{a}}$ Concentration of non-reactive propylene carbonate diluent in the resin.

${ }^{\mathrm{b}}$ Resin temperature during processing by stereolithography.

${ }^{\mathrm{c}}$ Degree of swelling in chloroform.

${ }^{\mathrm{d}}$ Melting peak at $40.7^{\circ} \mathrm{C}(\Delta \mathrm{H}=0.04 \mathrm{~J} / \mathrm{g})$ in the first DSC heating scan.

The glass transition temperatures of the extracted and dried PTMC networks are also given in Table 1. The onset and midpoint glass transition temperatures respectively ranged from -13.1 to $-19.0{ }^{\circ} \mathrm{C}$ and from -8.7 to $-17.0{ }^{\circ} \mathrm{C}$. These values are close to the glass transition temperatures previously found for PTMC networks crosslinked in the melt, indicating that these materials are flexible and rubber-like at room temperature. ${ }^{[14]}$ With increasing PTMC macromer molecular weight, the glass transition temperature of the resulting networks decreased. Furthermore, the temperature range of the glass transition became narrower. This indicates decreasing crosslinking densities. ${ }^{[17]}$ The networks obtained from PTMC 3250, PTMC 10300, PTMC 17150 and PTMC 20150 were completely amorphous. The network prepared from the PTMC 27750 resin showed a small melting endotherm at $40.7{ }^{\circ} \mathrm{C}(\Delta \mathrm{H}=0.04 \mathrm{~J} / \mathrm{g})$ in the first heating scan. In this case the low crosslinking density and the limited amount of entanglements due to the presence of diluent during the network formation process allowed the network chains to rearrange and partly crystallize. In tensile testing of non-crosslinked linear PTMC of very high molecular weights, strain-induced crystallization has been observed. Melting temperatures were between 30 and $50{ }^{\circ} \mathrm{C} .{ }^{[18-19]}$ 
Table 2. Tensile properties of extracted and dried crosslinked PTMC network films prepared by stereolithography.

\begin{tabular}{|c|c|c|c|c|c|c|}
\hline \multirow{2}{*}{$\begin{array}{l}\text { macromer } \\
\text { used to } \\
\text { prepare } \\
\text { network }^{\mathrm{a}}\end{array}$} & E-Modulus & $\sigma_{\text {yield }}$ & $\sigma_{\max }$ & $\varepsilon_{\text {yield }}$ & $\varepsilon_{\text {break }}$ & $\mathbf{W}^{\mathrm{b}}$ \\
\hline & MPa & MPa & MPa & $\%$ & $\%$ & $\mathbf{N} / \mathbf{m m}^{2}$ \\
\hline PTMC 3250 & $3.7 \pm 0.1$ & $0.40 \pm 0.02$ & $2.1 \pm 0.4$ & $14.5 \pm 0.7$ & $96 \pm 11$ & $97 \pm 26$ \\
\hline PTMC 10300 & $3.7 \pm 0.2$ & $0.64 \pm 0.01$ & $4.2 \pm 1.5$ & $30.8 \pm 1.2$ & $301 \pm 38$ & $449 \pm 138$ \\
\hline PTMC 17150 & $3.7 \pm 0.1$ & $1.04 \pm 0.05$ & $4.2 \pm 1.2$ & $50.6 \pm 3.4$ & $577 \pm 57$ & $971 \pm 225$ \\
\hline PTMC 20150 & $3.9 \pm 0.1$ & $1.17 \pm 0.02$ & $3.1 \pm 0.3$ & $57.7 \pm 2.5$ & $603 \pm 39$ & $933 \pm 137$ \\
\hline PTMC $27750^{\circ}$ & $4.2 \pm 0.2$ & $1.05 \pm 0.04$ & $1.3 \pm 0.1$ & $159.7 \pm 4.6$ & $991 \pm 40$ & $768 \pm 58$ \\
\hline
\end{tabular}

${ }^{\mathrm{a}}$ The resin formulations and temperatures during stereolithography are as given in Table 1 .

${ }^{\mathrm{b}}$ The toughness, $\mathrm{W}$ is determined as the area under the stress-strain curve.

${ }^{c}$ Specimen length was $55 \mathrm{~mm}$, grip-to-grip separation was $30 \mathrm{~mm}$. Since the elongation is derived from the grip-to-grip separation this leads to differing results.

The mechanical properties of the extracted and dried PTMC networks were assessed in tensile testing experiments at room temperature. Although the degrees of functionalization of the macromers differed, the mechanical properties of the obtained networks were mainly determined by the molecular weight of macromer used. ${ }^{[14]}$ In Table 2 an overview of relevant results is presented.

The table shows that all extracted networks are flexible and elastic materials with a low modulus of elasticity of approximately $4 \mathrm{MPa}$. The resilience of the networks prepared using the higher molecular weight PTMC macromers is illustrated by their very high elongations, with elongation at break values of more than $500 \%$, and high toughness values of more than $900 \mathrm{~N} / \mathrm{mm}^{2}$. In general, the yield strength, yield strain, maximal tensile strength and elongation at break of the networks increased with increasing macromer molecular weight. In the tensile testing of networks prepared from PTMC 27750 macromers a different gauge length was used. Since the deformation of the specimens was based on the grip-to-grip separation, the data for these materials given in Table 2 deviate from the other materials. Although these materials with low crosslinking densities showed small melting endotherms in the thermal analyses, strain hardening behavior was expected. Unfortunately, these specimens failed prematurely in our experiments.

When compared to similar PTMC networks prepared by photo-crosslinking in the melt in the absence of propylene carbonate diluent (Table 3), the elastic modulus values and the 
tensile strength values are somewhat lower. ${ }^{[14]}$ The elongations at break, however, were considerably higher. As mentioned before, these higher extensibilities are likely due to the supercontracted state of the network chains that results upon crosslinking in the diluted state after deswelling and drying. ${ }^{[16]}$ The toughnessess as determined from the areas under the stress-strain curves are relatively comparable.

Table 3. Tensile properties of extracted and dried crosslinked PTMC network films prepared by photo-crosslinking in the melt without the use of solvent. (taken from 14).

\begin{tabular}{|c|c|c|c|c|c|c|}
\hline \multirow{2}{*}{$\begin{array}{l}\text { macromer } \\
\text { used to } \\
\text { prepare } \\
\text { network }\end{array}$} & \multirow{2}{*}{$\frac{\text { E-Modulus }}{\mathbf{M P a}}$} & \multirow{2}{*}{$\frac{\sigma_{\text {yield }}}{\mathrm{MPa}}$} & \multirow{2}{*}{$\frac{\sigma_{\max }}{\mathrm{MPa}}$} & \multirow{2}{*}{$\frac{\varepsilon_{\text {yield }}}{\%}$} & \multirow{2}{*}{$\frac{\varepsilon_{\text {break }}}{\%}$} & \multirow{2}{*}{$\frac{\mathrm{W}}{\mathrm{N} / \mathbf{m m}^{2}}$} \\
\hline & & & & & & \\
\hline PTMC 3300 & $7.5 \pm 0.2$ & $0.62 \pm 0.09$ & $3.5 \pm 0.4$ & $8 \pm 0.4$ & $78 \pm 6$ & $131 \pm 20$ \\
\hline PTMC 10300 & $4.5 \pm 0.2$ & $0.78 \pm 0.01$ & $3.7 \pm 0.4$ & $26 \pm 0.1$ & $244 \pm 9$ & $426 \pm 38$ \\
\hline РTMC 18900 & $5.8 \pm 0.1$ & $1.24 \pm 0.01$ & $7.5 \pm 1.4$ & $34 \pm 3$ & $425 \pm 31$ & $1333 \pm 282$ \\
\hline PTMC 20000 & $4.3 \pm 0.1$ & $1.17 \pm 0.01$ & $5.4 \pm 0.2$ & $44 \pm 2$ & $467 \pm 14$ & $1107 \pm 59$ \\
\hline PTMC 24600 & $5.2 \pm 0.2$ & $1.46 \pm 0.02$ & $9.8 \pm 1.4$ & $48 \pm 2$ & $822 \pm 29$ & $2786 \pm 157$ \\
\hline
\end{tabular}

When these PTMC networks are to be used as biomaterials in vivo, their surface hydrophilicities and water uptake properties are of great importance. These properties strongly influence the interactions of cells and tissues with a biomaterial, and also can affect their mechanical properties and degradation behavior upon implantation.

It is shown in Figure 3 that the photo-crosslinked PTMC networks films were rather hydrophobic. Independent of the macromer molecular weight used in the network preparation, the equilibrium water uptake at room temperature was $1.1 \pm 0.1 \%$. The hydrophobic nature of extracted and dried PTMC networks is also reflected by the static water contact angles which varied between 89 and $98^{\circ}$. Upon equilibration in water, however, the water contact angle decreases. The surface of a network prepared from a PTMC macromer with molecular weight of $3050 \mathrm{~g} / \mathrm{mol}$ initially has a static contact angle of $93 \pm 2^{\circ}$ in the dry state, after conditioning in water this is reduced to $61 \pm 3^{\circ} \cdot[13]$ 


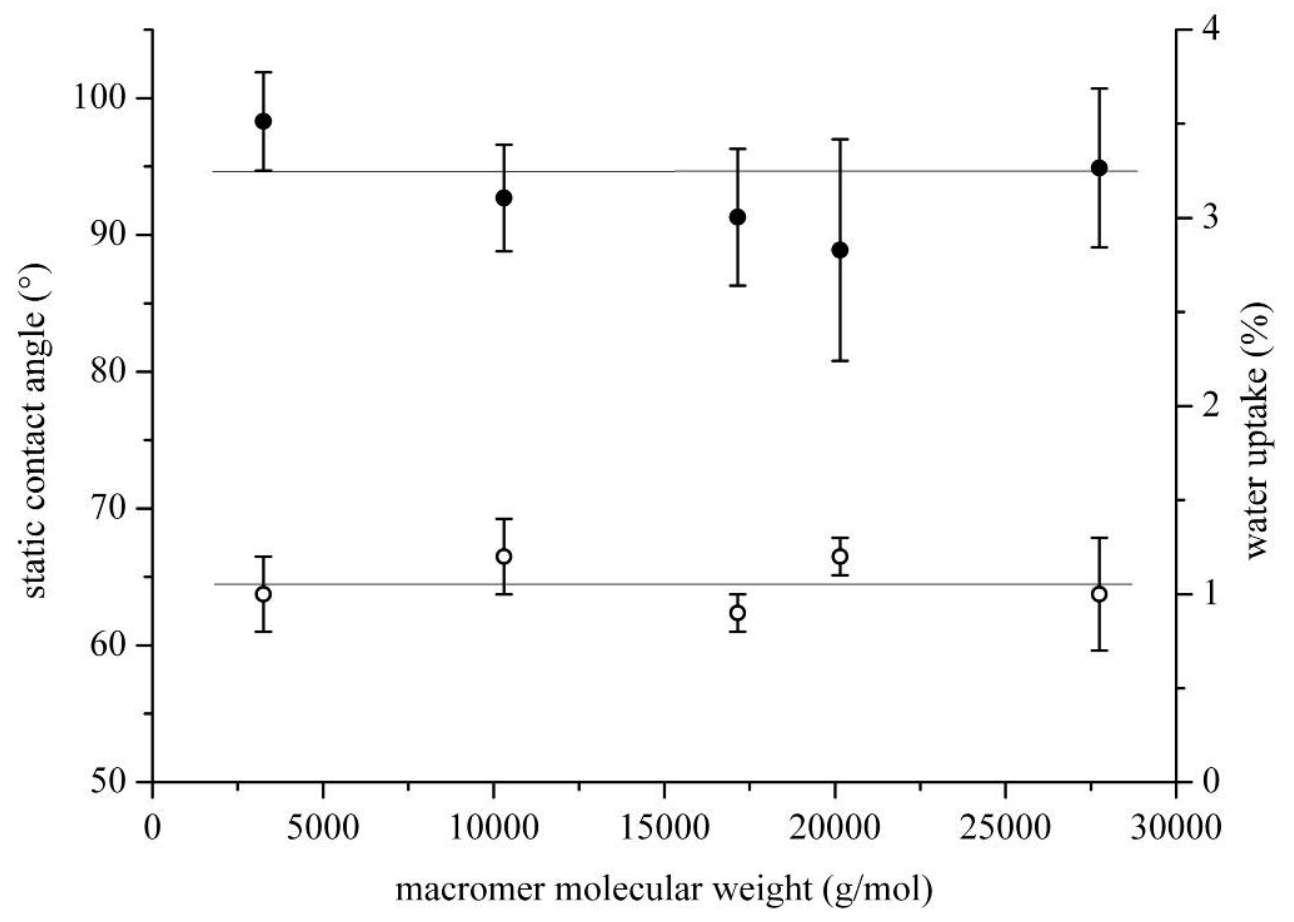

Figure 2. Water uptake (०) and static water contact angles $(\bullet)$ of extracted and dried PTMC network films prepared by stereolithography using PTMC macromers of different molecular weights. The resin formulations and temperatures during stereolithography are as indicated in Table 1.

\section{Designed Three-Dimensional PTMC Structures Prepared by Stereolithography}

Although stereolithography is perhaps not the best method to prepare photo-crosslinked films, it does allow the preparation of three-dimensional structures according to advanced designed architectures. In tissue engineering this can be highly beneficial, as scaffolds can be obtained with pore structures that facilitate cell seeding and proliferation having fully interconnected pore network structures with narrow pore size distributions.

It follows from the preceding part, that networks prepared from macromers with the higher molecular weights are likely to have optimal physical- and mechanical properties for the preparation of biomedical implants and devices. However, it can be anticipated that the resins based on these macromers will be the most difficult to process into three-dimensional structures by stereolithography, as these will contain the highest amounts of diluent.

The resins containing PTMC macromers of molecular weights between 3250 and $27750 \mathrm{~g} / \mathrm{mol}$ were diluted with different amounts of propylene carbonate and photo-crosslinked in the stereolithography apparatus to give solid cylinders with a diameter 
of $4.8 \mathrm{~mm}$ and a height of $6.3 \mathrm{~mm}$ after extraction and drying. In designing the cylinders, the amount of non-reactive diluent was taken into consideration. The resin based on PTMC 3250 was processed at room temperature after dilution with $30 \mathrm{wt} \%$ propylene carbonate, the other resins based on higher molecular weight macromers contained 22 to $50 \mathrm{wt} \%$ diluent and were processed at $65{ }^{\circ} \mathrm{C}$. It should be noted that during the building process the temperature in these cases increased to approximately $70{ }^{\circ} \mathrm{C}$. This is likely due to the intensity of the light and the exothermic nature of the polymerization reaction. ${ }^{[4]}$ An overview of the applied stereolithography resins and processing conditions is given in Table 4.

Table 4. Characteristics of the PTMC resins used and of the designed solid cylinders built by stereolithography after extraction and drying.

\begin{tabular}{|c|c|c|c|c|c|}
\hline \multirow{2}{*}{$\begin{array}{l}\text { macromer } \\
\text { used to } \\
\text { prepare } \\
\text { network }^{\text {a }}\end{array}$} & $\begin{array}{c}\text { diluent } \\
\text { concentration }\end{array}$ & temperature $^{\mathrm{b}}$ & $\begin{array}{l}\text { cylinder } \\
\text { diameter }\end{array}$ & shrinkage $^{c}$ & $\mathbf{E}^{\mathrm{d}}$ \\
\hline & wt $\%$ & ${ }^{\circ} \mathbf{C}$ & $\mathbf{m m}$ & $\%$ & MPa \\
\hline PTMC 3250 & 30 & 22 & $4.57 \pm 0.05$ & $22.7 \pm 0.6$ & $4.2 \pm 0.2$ \\
\hline PTMC $10300^{\mathrm{e}}$ & 22 & 65 & $5.07 \pm 0.03$ & $21.5 \pm 0.3$ & $3.1 \pm 0.3$ \\
\hline PTMC 17150 & 40 & 65 & $4.50 \pm 0.02$ & $23.9 \pm 0.6$ & $3.1 \pm 0.1$ \\
\hline PTMC 20150 & 45 & 65 & $4.94 \pm 0.03$ & $31.1 \pm 0.4$ & $3.8 \pm 0.1$ \\
\hline PTMC $27750^{\mathrm{f}}$ & 53 & 65 & - & - & - \\
\hline
\end{tabular}

\footnotetext{
${ }^{a}$ Resin contained PTMC macromers, non-reactive propylene carbonate diluent, photo-initiator and dye.

${ }^{\mathrm{b}}$ Initial resin temperature during processing by stereolithography.

${ }^{c}$ Shrinkage due to presence of non-reactive diluent, polymerization reaction and building at elevated temperature.

${ }^{\mathrm{d}}$ Elasticity moduli were determined in compression experiments.

${ }^{\mathrm{e}}$ Resin formulation and processing temperature differ from resin used in preparing films (Table 1).

${ }^{\mathrm{f}}$ Not possible to build cylindrical structures.
}

The solid cylinders could readily be prepared using the resins based on PTMC macromers with molecular weights of $20150 \mathrm{~g} / \mathrm{mol}$ and lower. As Figure 1 shows, the amount of nonreactive propylene carbonate diluent in the resin which is needed to sufficiently reduce the viscosity increases with increasing molecular weight of the PTMC macromers. It was found that resins based on the highest macromer molecular weights were the most difficult to process properly and careful optimization was needed. It was not even possible to successfully build three-dimensional structures in our stereolithography setup using the 
PTMC 27750 resin as the (swollen) networks obtained upon crosslinking were too fragile and brittle.

As shown in Table 4, the diameters of the cylindrical structures varied between 4.5 and $5.1 \mathrm{~mm}$ after extraction and drying. Their heights were $6.22 \pm 0.45 \mathrm{~mm}$. As the non-reactive diluent is removed upon extraction and drying, the cylinders will shrink. Because this shrinkage is isotropic, it can be taken into account in the design of the structure that is to be built. In our work this was done, and the solid cylinders were designed to have a diameter of $4.8 \mathrm{~mm}$ and a height of $6.3 \mathrm{~mm}$ after extraction and drying. However, the shrinkage is additionally influenced by the formation of a crosslinked network from oligomeric compounds and the cooling of the formed specimens from the high temperatures at which the resins were crosslinked to room temperature. The shrinkage of the crosslinked networks, determined by comparing the dimensions of the built (=designed) structures with the dimensions of the structures after extraction and drying, varied between 21 and $31 \%$.

The mechanical properties of the solid PTMC cylinders built by stereolithography were evaluated in compression tests after extraction and drying. The elastic moduli of the networks were very similar to those determined in tensile testing (Table 2) and varied between 3.1 and 4.2 MPa. No significant differences were seen for networks prepared from PTMC macromers with different molecular weights. After compressing the cylinders to $65-75 \%$, the specimens returned to at least $98 \%$ of their original heights within $30 \mathrm{~min}$. This illustrates the highly elastic nature of the photo-crosslinked PTMC networks.

Designed porous cylindrical structures were also prepared by stereolithography using the different PTMC resins. The porous cylinders were designed to have an interconnected pore network with gyroid architecture. ${ }^{[3,5]}$ Taking the amount of non-reactive propylene carbonate diluent into account, the cylinders were designed to have the same external dimensions as the solid cylinders (diameter $=4.8 \mathrm{~mm}$, height $=6.3 \mathrm{~mm}$ ) and a porosity of $71 \%$ with an average pore size of $442 \mu \mathrm{m}$.

As was also the case for solid cylinders, designed porous cylinders with a gyroid pore network architecture could be prepared using the resins based on PTMC macromers with molecular weights between 3250 and $20150 \mathrm{~g} / \mathrm{mol}$. Figure 3 visualizes an example of a structure with gyroid pore architecture prepared by stereolithography using a PTMC 3250 resin. Figure $3 \mathrm{~A}$ is a photograph of the structure after extraction and drying. It can be seen that here too the shrinkage is isotropic, and that the designed structure is preserved after removal of the diluent and drying. In Figure $3 \mathrm{~B}$ a $\mu \mathrm{CT}$ image is presented of the same 
specimen, showing that the porosity of the structure is highly regular and fully interconnected. In Figures 3C and 3D high resolution SEM images are presented of respectively the outer side of the porous PTMC structure and of a cross-section. These images confirm the highly regular open porosity of the porous structures. Also evident from the images is the structuring of the specimens which reveals the layer-by-layer manner in which the constructs are built by stereolithography.
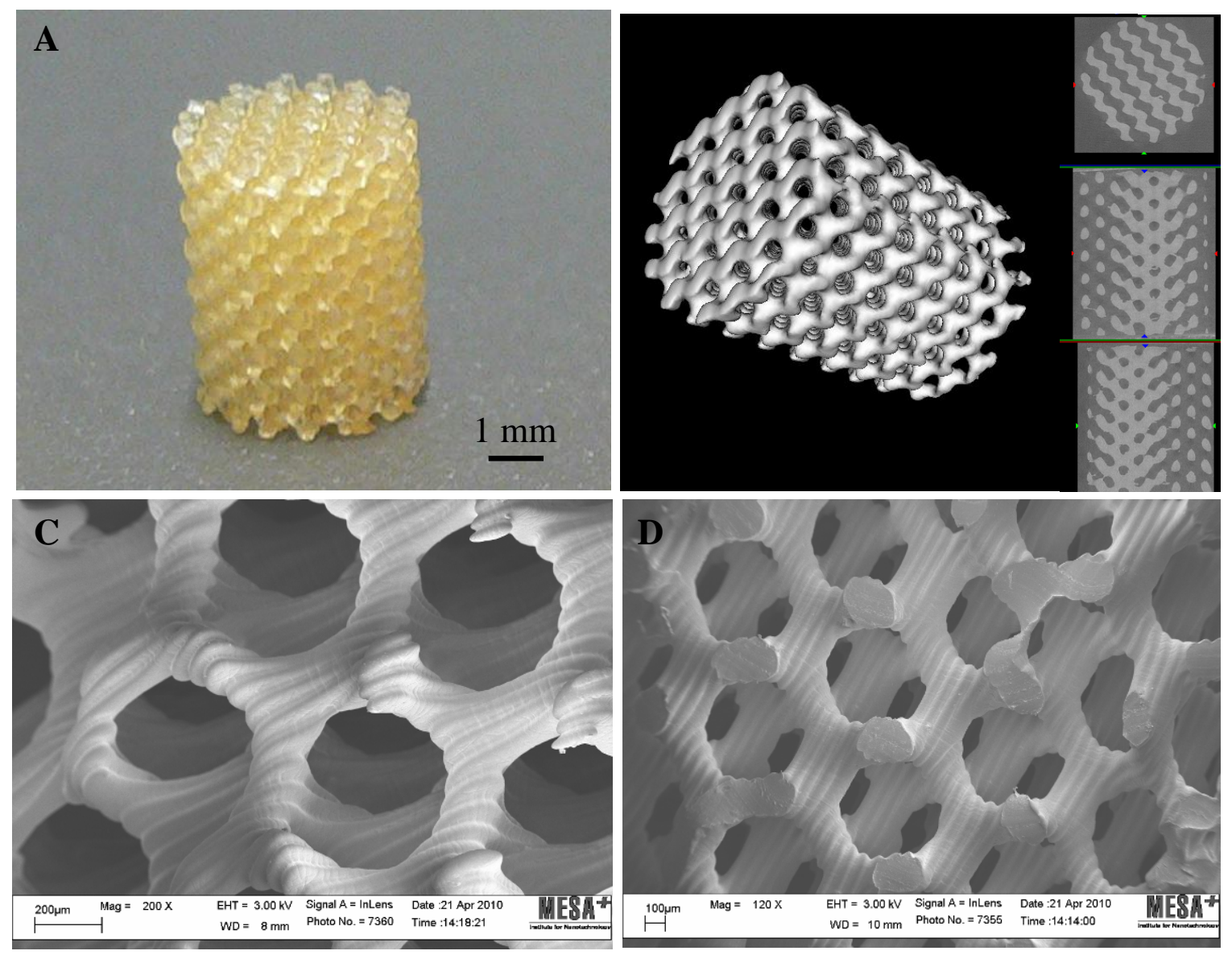

Figure 3. Designed three-dimensional porous structures with a gyroid pore network architecture prepared by stereolithography using a PTMC 3250 resin. Photographic image (A), $\mu C T$ visualization (B) and high resolution SEM images showing the outer side $(C)$ and a cross-section (D) of the built structure.

Taking the amount of non-reactive propylene carbonate diluent into consideration, the porous gyroid structures were designed to have the same external dimensions as the solid cylinders and a porosity of $71 \%$ with an average pore size of $442 \mu \mathrm{m}$. It was found that the shrinkage (after extraction and drying) based on the external dimensions of the built 
specimens was similar to that of the solid cylinders previously described, yielding porous structures having diameters between 4.48 and $5.02 \mathrm{~mm}$.

Table 5. Characteristics of the PTMC resins used and of the designed porous cylinders with gyroid network architecture built by stereolithography after extraction and drying.

\begin{tabular}{|c|c|c|c|}
\hline \multirow{2}{*}{$\begin{array}{l}\text { macromer used to } \\
\text { prepare network }^{\text {a }}\end{array}$} & porosity $^{b}$ & average pore size $^{b}$ & $\mathbf{E}^{\mathrm{c}}$ \\
\hline & $\%$ & $\mu \mathrm{m}$ & kPa \\
\hline PTMC 3250 & $66 \pm 1$ & $378 \pm 8$ & $20.4 \pm 4.3$ \\
\hline PTMC 10300 & $62 \pm 2$ & $407 \pm 11$ & $42.5 \pm 8.9$ \\
\hline PTMC 17150 & $53 \pm 2$ & $309 \pm 8$ & $156.0 \pm 41.9$ \\
\hline PTMC 20150 & $56 \pm 1$ & $339 \pm 7$ & $108.8 \pm 18.4$ \\
\hline PTMC $27750^{d}$ & - & - & - \\
\hline
\end{tabular}

${ }^{\text {a }}$ Resin composition and stereolithography processing conditions as given in Table 3.

${ }^{\mathrm{b}}$ Porosity and average pore sizes were determined from $\mu \mathrm{CT}$ data. Average \pm standard deviation $(\mathrm{n}=5)$.

${ }^{c}$ Elasticity moduli of the porous structures were determined in compression experiments.

${ }^{\mathrm{d}}$ Not possible to build designed porous cylindrical structures.

In Table 5 an overview is given of the porosities and the average pore sizes of structures prepared from the different PTMC macromer resins as determined from the $\mu \mathrm{CT}$ analyses. All porous structures show lower porosities and pore sizes than was designed, the determined porosity values varied between 53 and $66 \%$, and the average pore sizes between 309 and $407 \mu \mathrm{m}$. This can be the result of additional shrinkage due to the crosslinked nature of the networks and their cooling to room temperature, but can also be the result of overcuring. As during the building process the resin can heat up locally due to the high light intensity and the exothermic polymerization reaction of the resin, the depth of cure could have been higher than anticipated from the stereolithography working curves. ${ }^{[4]}$

In building these designed porous structures by stereolithography, it was also found that the molecular weight of the PTMC macromer, which determines the necessary amount of diluent in the resin, to a large extent determines whether the porous structures can be built. The fragility of the swollen networks during building is due to the presence of relatively large amounts of diluent and the low crosslink density of the networks prepared from the macromers with the highest molecular weights. The designed porous structures could not be built using the resin based on PTMC 27750, and resins based on PTMC macromers with 
the higher molecular weights could only be built after minimization of diluent contents, and meticulous optimization of building temperatures and illumination times in the stereolithography process.

The mechanical properties, and especially the elastic modulus, of porous structures are strongly dependent on the porosity of the structures. It can be seen in Table 5 that the compression moduli of the extracted and dried porous PTMC network structures prepared from the different macromers are significantly lower than those of the corresponding solid specimens (see Table 4). While the different solid PTMC network specimens have comparable E-modulus values close to $4 \mathrm{MPa}$, the compression modulus values of the stereolithography built porous structures strongly depend on porosity. The determined values vary from approximately $20 \mathrm{kPa}$ for the structures with the highest porosities of $66 \%$ to $156 \mathrm{kPa}$ for specimens with a porosity of $53 \%$.

\section{Conclusions}

Photo-crosslinkable PTMC macromers were prepared by ring opening polymerization of trimethylene carbonate and end-functionalization with methacrylate groups. Resins based on PTMC macromers with molecular weights between 3250 and $27750 \mathrm{~g} / \mathrm{mol}$ were used in stereolithography to prepare designed structures. To allow processing by stereolithography, it was found necessary to add non-reactive propylene carbonate as diluent to the resin and to increase the stereolithography building temperature to $65^{\circ} \mathrm{C}$. Designed three-dimensional porous and non-porous structures were successfully built by stereolithography using resins based on PTMC macromers with molecular weights lower than $20150 \mathrm{~g} / \mathrm{mol}$ that contained up to $45 \mathrm{wt} \%$ propylene carbonate.

It was found that the resins based on the PTMC macromers with the highest molecular weights were the most difficult to process, as these yielded the weakest and most fragile structures upon photo-crosslinking. The swollen networks formed using these macromers necessarily contained the largest amounts of diluent and also had the lowest crosslinking densities. The mechanical properties of the structures were determined in tensile testing experiments and in compression tests. It was found that after extraction and drying, the networks were elastic and flexible with elastic moduli of approximately $4 \mathrm{MPa}$. Tensile strength, elongation at break and toughness of the networks increased with increasing molecular weight of the PTMC macromer used. 
The use of resins based on PTMC macromers in stereolithography allows the preparation of biodegradable designed porous structures with well-defined porosities, pore network architectures and suitable mechanical properties for applications in medicine and tissue engineering.

\section{References}

[1] Melchels FPW, Feijen J, Grijpma DW. A review on stereolithography and its applications in biomedical engineering. Biomaterials 2010, 31, 6121-6130.

[2] Savalani MM, Harris RA. Layer manufacturing for in vivo devices. P I Mech Eng H 2006, 220, 505-520.

[3] Melchels FPW, Feijen J, Grijpma DW. A poly(D,L-lactide) resin for the preparation of tissue engineering scaffolds by stereolithography. Biomaterials 2009, 30, 38013809.

[4] Melchels FPW, Bertoldi K, Gabbrielli R, Velders AH, Feijen J, Grijpma DW. Mathematically defined tissue engineering scaffold architectures prepared by stereolithography. Biomaterials 2010, 31, 6909-6916.

[5] Cooke MN, Fisher JP, Dean D, Rimnac C, Mikos AG. Use of stereolithography to manufacture critical-sized 3D biodegradable scaffolds for bone ingrowth. J Biomed Mater Res 2003, 64B, 65-69.

[6] Lee JW, Lan PX, Kim B, Lim G, Cho D-W. 3D scaffold fabrication with PPF/DEF using micro-stereolithography. Microelectron Eng 2007, 84, 1702-1705.

[7] Dhariwala B, Hunt E, Boland T. Rapid prototyping of tissue-engineerign constructs, using photopolymerizable hydrogels and stereolithography. Tissue Eng 2004, 10, 1316-1322.

[8] Mapili G, Lu Y, Chen S, Roy K. Laser-layered microfabrication of spatially patterned functionalized tissue-engineering scaffolds. J Biomed Mater Res B 2005, 75B, 414424.

[9] Jansen J, Melchels FPW, Grijpma DW, Feijen J. Fumaric acid monoethyl esterfunctionalized poly(D,L-lactide)/N-vinyl-2-pyrrolidone resins for the preparation of tissue engineering scaffolds by stereolithography. Biomacromolecules 2009, 10, 214220.

[10] Matsuda T, Mizutani M. Liquid acrylate-endcapped biodegradable $\operatorname{poly}(\varepsilon-$ caprolactone-co-trimethylene carbonate). II. Computer-aided stereolithographic microarchitectural surface photoconstructs. J Biomed Mater Res 2002, 62, 395-403.

[11] Kwon KI, Matsuda T. Photo-polymerized microarchitectural constructs prepared by microstereolithography $(\mu \mathrm{SL})$ using liquid acrylate-end-capped trimethylene carbonate-based prepolymers. Biomaterials 2005, 26, 1675-1684.

[12] Lee S-J, Kang H-W, Park JK, Rhie J-W, Hahn SK, Cho D-W. Application of microstereolithography in the development of three-dimenstional cartilage regeneration scaffolds. Biomed Microdevices 2008, 10, 233-241.

[13] Chapter 6 of this thesis.

[14] Chapter 3 of this thesis.

[15] Hildebrand T, Ruegsegger P. A new method for the model-independent assessment of thickness in three-dimensional images. J Microscopy-Oxford 1997, 185, 67-75. 
[16] Premachandra JK, Mark JE. Effects of dilution during crosslinking on strain-induced crystallization in cis-1,4-polyisoprene networks. I. Experimental results. J. Macromol. Sci.-Pure Appl. Chem. A 2002, 39(4), 287-300.

[17] Ellis TS, Karasz FE, Ten Brinke G. The influence of thermal-properties on the glasstransition temperature in styrene/divinylbenzene network diluent systems. $J$ Appl Polym Sci 1983, 28, 23-32.

[18] Pêgo AP, Poot AA, Grijpma DW, Feijen J. Copolymers of trimethylene carbonate and e-caprolactone for porous nerve guides: synthesis and properties. J Biomater Sci Polym Ed 2001, 12, 35-53.

[19] Pêgo AP, Grijpma DW, Feijen J. Enhanced mechanical properties of 1,3-trimethylene carbonate polymers and networks. Polymer 2003, 44, 6495-6504. 


\title{
Chapter 6
}

\section{Designed Scaffolds for Cartilage Tissue Engineering Prepared by Stereolithography Using Poly(Trimethylene Carbonate)-Based Resins}

\begin{abstract}
The aim of this research was to investigate the applicability of designed poly(trimethylene carbonate) (PTMC) structures prepared by stereolithography as scaffolds for cartilage tissue engineering. A three-armed methacrylated PTMC macromer with a molecular weight of $3100 \mathrm{~g} / \mathrm{mol}$ was formulated into a resin, which upon photo-crosslinking yielded network films with a tensile strength of $1.7 \pm 0.1 \mathrm{MPa}$, E-modulus of 3.7 $\pm 0.4 \mathrm{MPa}$ and toughness of $76 \pm 7 \mathrm{~N} / \mathrm{mm}^{2}$. The static water contact angle was $93 \pm 2^{\circ}$, which decreased to $61 \pm 3^{\circ}$ upon equilibration of the network films in water.

The resin was used to build designed scaffolds with a gyroid pore architecture by means of stereolithography. Highly interconnected porous structures were prepared with an average pore diameter of $350 \pm 12 \mu \mathrm{m}$ and a porosity of $54.0 \pm 2.2 \%$. Bovine chondrocytes were seeded in the scaffolds and were found to adhere and spread on the PTMC surface. After culturing for 6 weeks also cells with a round morphology were present, indicative of the differentiated chondrocyte phenotype, adhering to the spread cells. Sulphated glycosaminoglycans and fibrillar collagens were deposited by the cells. During culturing for 6 weeks, the compression moduli of the constructs increased 50\% to approximately $100 \mathrm{kPa}$. In conclusion, designed PTMC scaffolds suitable for seeding of chondrocytes were built by stereolithography. Optimization of chondrocyte re-differentiation upon seeding will further increase the applicability of these constructs for cartilage tissue engineering.
\end{abstract}




\section{Introduction}

Due to the increase in life expectancy, degenerative disease and trauma, there is an increasing demand for strategies to restore the function of articular cartilage. Current methods for cartilage repair include the use of cell-based therapies, intended to regenerate the damaged tissue on a bioresorbable three-dimensional support structure. Under suitable culture conditions or after direct implantation into the patient, autologous chondrocytes should proliferate in the scaffold and deposit cartilage-specific extracellular matrix. Several efforts have been carried out in this direction, predominantly using hydrogels as substrates. $^{[1,2]}$ Although hydrogels can mimic a three-dimensional environment for cell attachment, their mechanical properties remain a challenge. ${ }^{[3]}$

Photo-polymerizable and photo-crosslinkable polymers that yield flexible and elastic materials receive increasing attention in the field of tissue engineering. ${ }^{[4,5]}$ Processing of these polymers by stereolithography would produce flexible and elastic three-dimensional structures. By means of stereolithography, three-dimensional structures are created layer by layer, irradiating a photo-sensitive resin according to a given design. ${ }^{[6-8]}$ The control of critical parameters such as porosity, pore size, pore size distribution and pore interconnectivity, promotes the transport of nutrients and metabolic waste products throughout the scaffold. ${ }^{[7,9]}$ Application of stereolithography in tissue engineering presents additional advantages over conventional approaches, since patient-specific implants can be constructed on the basis of scanning data of magnetic resonance imaging or tomography techniques. $^{[10]}$

We recently reported on the application of PTMC macromers in stereolithography. ${ }^{[1]}$ The resulting PTMC network structures are flexible and elastic. The mechanical properties of photo-crosslinked PTMC depend on the molecular weight of the macromer, allowing the creation of structures with desired modulus, elongation and strength. ${ }^{[12]}$ In addition, PTMC networks show good compatibility with different cell types and are enzymatically degradable. ${ }^{[13-15]}$

In the present study we aim to investigate the ability of PTMC scaffolds built by stereolithography to support the attachment and proliferation of primary bovine chondrocytes. To evaluate cell morphology and deposition of extracellular matrix, microscopical and histological analyses were performed during a culture period of 6 weeks. Moreover, the effect of chondrocyte culturing on the mechanical properties of the constructs was investigated by dynamic mechanical analysis. 


\section{Materials and Methods}

\section{Preparation of PTMC Resins}

A three-armed poly(trimethylene carbonate) (PTMC) macromer was synthesized in a twostep reaction and formulated into a liquid resin to be applied in the stereolithography apparatus. In the first step, hydroxyl-terminated PTMC oligomer was prepared by ring opening polymerization (oligomerization) of 1,3-trimethylene carbonate (TMC, Boehringer Ingelheim, Germany) using glycerol (Aldrich, USA; dehydrated by briefly heating at $180{ }^{\circ} \mathrm{C}$ ) as initiator and $0.07 \mathrm{wt} \% \mathrm{SnOct}_{2}$ (Sigma, USA) as catalyst on a $125 \mathrm{~g}$ scale. The TMC monomer and glycerol initiator were charged into a freshly silanized and dried (Serva silicone solution, SERVA Electrophoresis GmbH, Germany) three-necked flask and polymerized in an argon atmosphere at $130{ }^{\circ} \mathrm{C}$ for 5 days. Subsequently, the hydroxylterminated oligomer was functionalized by reaction with methacrylic anhydride (Aldrich, Germany), applied at an excess of $50 \mathrm{~mol} \%$ (per functional hydroxyl group) at $120{ }^{\circ} \mathrm{C}$ for 2 days. To avoid premature crosslinking, $0.06 \mathrm{wt} \%$ hydroquinone (Aldrich, Germany) and $0.06 \mathrm{wt} \%( \pm)-\alpha$-tocopherol (Fluka, Switzerland) were added during this reaction. The obtained macromer was purified by distillation of the formed methacrylic acid and the excess methacrylic anhydride under vacuum up to a temperature of $180{ }^{\circ} \mathrm{C}$ $\left(\mathrm{T}_{\text {vapor }}=105-110^{\circ} \mathrm{C}\right)$. The monomer conversion of the oligomerization reaction, the molecular weight and the degree of functionalization were determined by ${ }^{1} \mathrm{H}-\mathrm{NMR}$ spectroscopy (Varian Innova $300 \mathrm{MHz}$, USA), using $\mathrm{CDCl}_{3}$ (Merck, Germany) as solvent.

To apply a macromer in the stereolithography apparatus it has to be formulated into a liquid resin with a viscosity of approximately $10 \mathrm{~Pa} \cdot \mathrm{s} .{ }^{[11]}$ The PTMC resin was prepared by adding $22 \mathrm{wt} \%$ propylene carbonate (Merck, Germany) to the macromer followed by addition of $5 \mathrm{wt} \%$ Lucirin TPO-L (BASF, Germany) and $0.15 \mathrm{wt} \%$ Orasol Orange G dye (Ciba Specialty Chemicals, Switzerland). The latter two weight percentages are relative to the macromer. It should be noted that films for tensile testing experiments were prepared from a resin without Orasol Orange G. The resin viscosity was determined at temperatures between 20 and $25{ }^{\circ} \mathrm{C}$, using a Brookfield DV-E rotating spindle viscometer. The viscometer was equipped with a small sample adapter and the Brookfield s21 spindle, which was operated at shear rates of 2.79 and $3.721 / \mathrm{s}$ (rotating at 3 and $4 \mathrm{rpm}$, respectively). 


\section{Preparation of PTMC Films and Scaffolds by Stereolithography}

An EnvisionTec Perfactory Mini Multilens (Germany) stereolithography apparatus was used to build flat films and three-dimensional scaffolds from the PTMC-based resins. The stereolithography apparatus is equipped with a digital micro-mirror device, allowing projections of $1280 \times 1024$ pixels, each measuring $32 \times 32 \mu \mathrm{m}^{2} .{ }^{[16]}$ By applying the Enhanced Resolution Module (ERM) technology, the $\mathrm{x}$ and $\mathrm{y}$ resolution of the apparatus can be doubled, yielding pixels measuring $16 \times 16 \mu \mathrm{m}^{2}{ }^{217]}$ The wavelength of the (blue) light in the stereolithography apparatus ranges between 400 to $550 \mathrm{~nm}$, with a peak at $440 \mathrm{~nm} .^{[18]}$

Flat films for tensile testing were prepared by illuminating a rectangular area of the resin measuring $75 \times 60 \mathrm{~mm}^{2}$ for $5 \mathrm{~min}$ at an intensity of approximately $10 \mathrm{~mW} / \mathrm{cm}^{2}$ (lens $\mathrm{f}=60 \mathrm{~mm}$, without ERM). The layer thickness was $0.55 \mathrm{~mm}$. The films were subsequently post-cured by irradiation for $30 \mathrm{~min}$ in a crosslinking cabinet (Ultralum, USA, operating at $365 \mathrm{~nm}$ and an intensity of $3-4 \mathrm{~mW} / \mathrm{cm}^{2}$ ) in a nitrogen atmosphere. The resulting films were extracted with a 1:1 mixture of propylene carbonate and ethanol, which was refreshed twice after which the films were washed with ethanol and dried under vacuum until constant weight.

A cylindrical three-dimensional scaffold with gyroid pore architecture was designed using K3dSurf v0.6.2 (freeware from http://k3dsurf.sourceforge.net) and Rhinoceros 3D (McNeel Europe) computer software. The scaffold was designed to have a porosity of $71 \%$, an average pore size of $547 \mu \mathrm{m}$, a diameter of $5.94 \mathrm{~mm}$ and a height of $7.74 \mathrm{~mm}$ (Figure 1).

The three-dimensional structures were built by stereolithography at a pixel resolution of $16 \times 16 \mu \mathrm{m}^{2}$ and layer thicknesses of $25 \mu \mathrm{m}$. Subsequent layers were illuminated at an intensity of approximately $17 \mathrm{~mW} / \mathrm{cm}^{2}$ for $65 \mathrm{~s}$ (lens $\mathrm{f}=85 \mathrm{~mm}$, with ERM). In order to precisely control the penetration depth of the light when preparing the designed structure, a small amount of Orasol Orange dye was added to the resin, as described above. The structures were then carefully extracted with propylene carbonate for one week, after which small additions of ethanol allowed the structures to slowly shrink to their final dimensions in the course of several days. Finally, the scaffolds were washed with ethanol and dried under vacuum until constant weight. 


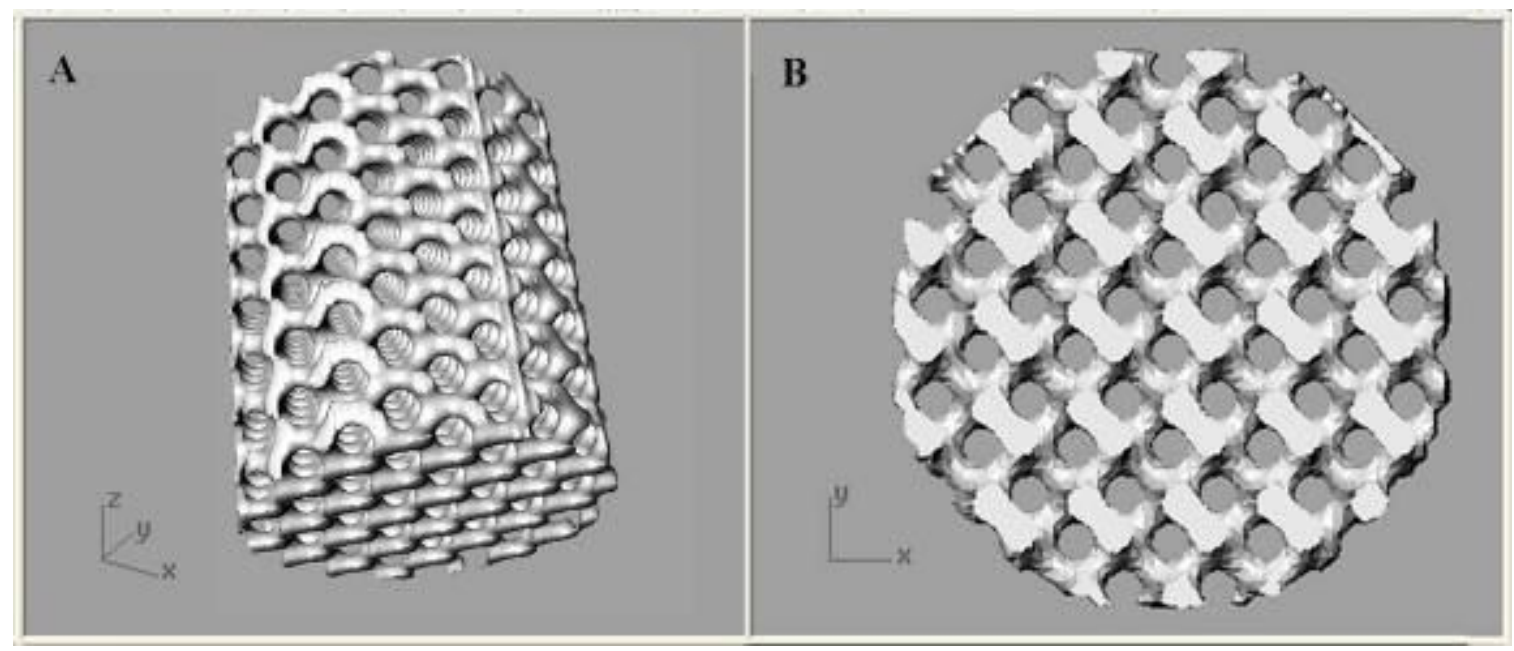

Figure 1. Design of the porous scaffold (diameter $=5.94 \mathrm{~mm}$, height=7.74 $\mathrm{mm}$ ) with gyroid pore architecture having an average pore size of $547 \mu m$ and a porosity of $71 \%$. Perspective (A) and top-view (B).

\section{Characterization of PTMC Films and Scaffolds}

The network characteristics and the physical properties of the PTMC networks prepared by stereolithography were assessed using the flat film specimens after extraction and drying. The volume degree of swelling was assessed at room temperature by swelling disk-shaped specimens ( $8 \mathrm{~mm}$ in diameter, punched out from the extracted films) in chloroform for 2 days. The values were determined as described previously and given as average \pm standard deviation $(n=3) .{ }^{[1]}$

To determine their water uptake, disks of $8 \mathrm{~mm}$ in diameter were punched out of the network films and equilibrated in ultra-pure water for $45 \mathrm{~h}$ at room temperature. The values were determined as described previously and given as average \pm standard deviation $(\mathrm{n}=3)^{[11]}$

Static contact angles of ultra-pure water (MilliQ Plus, Millipore, France) on the surface of a dry PTMC network film were determined using a video-based OCA 20 contact angle system from DataPhysics (Germany), equipped with an electronic syringe module. The static water contact angle was also determined on a wet PTMC network film after equilibration in water for several days. The measurements were performed at room temperature on five different regions of a film. The presented values are given as average \pm standard deviation. 
The thermal properties of the PTMC networks were determined by differential scanning calorimetry (DSC) using a Perkin Elmer Pyris 1 (USA) calibrated with cyclohexane, indium and lead standards. A sample of approximately $10 \mathrm{mg}$ was heated from $-100{ }^{\circ} \mathrm{C}$ to $100{ }^{\circ} \mathrm{C}$ at a heating rate of $10{ }^{\circ} \mathrm{C} / \mathrm{min}$ and quenched rapidly $\left(300{ }^{\circ} \mathrm{C} / \mathrm{min}\right)$ to $-100{ }^{\circ} \mathrm{C}$. After 5 min, a second scan was recorded from which the glass transition temperature $\left(\mathrm{T}_{\mathrm{g}}\right)$ was determined as midpoint value of the heat capacity change.

The mechanical properties of the PTMC network films were determined at room temperature using a Zwick Z020 tensile tester (Germany), equipped with a $500 \mathrm{~N}$ load cell according to ASTM D882-91 at a cross-head speed of $50 \mathrm{~mm} / \mathrm{min}$ using specimens measuring $66 \times 5 \mathrm{~mm}^{2}$. The elongation of the specimens was derived from the grip-to-grip separation, which initially was $50 \mathrm{~mm}$. The E-modulus was determined from the initial slope of the stress-strain curves. The yield stress and the elongation at yield were determined from the intersection of tangents to the stress-strain curves. The toughness of the specimens $\left(\mathrm{W}\right.$ in $\mathrm{N} / \mathrm{mm}^{2}$ ) was determined as the area under the stress-strain curves. Experiments were conducted in triplicate.

Micro-computed tomography $(\mu \mathrm{CT}$ ) (GE eXplore Locus SP $\mu \mathrm{CT}$ scanner with a spatial resolution of $7 \mu \mathrm{m}$ ) was employed to visualize the porous structures built by stereolithography. Porosities, pore size distributions and average pore sizes of six different scaffolds were assessed from the $\mu \mathrm{CT}$ data as described elsewhere. ${ }^{[18,19]}$ All values are presented as average \pm standard deviation. Humid constructs were also analyzed by $\mu \mathrm{CT}$ after seeding and culturing of chondrocytes. The porous scaffolds were visualized as well by scanning electron microscopy (SEM) (Philips XL30 ESEM-FEG, The Netherlands). The specimens were sputter-coated with gold and the apparatus was operated at a voltage of $10 \mathrm{kV}$.

Dynamic mechanical properties of scaffolds were determined in the dry and wet state using a dynamic mechanical analyzer (Perkin Elmer 7E, USA). A static force of $500 \mathrm{~N}$ was applied on which a dynamic force of $250 \mathrm{~N}$ was superimposed at a constant frequency of $1 \mathrm{~Hz}$. The compression modulus was recorded between 20 and $40{ }^{\circ} \mathrm{C}$ at a heating rate of $1{ }^{\circ} \mathrm{C} / \mathrm{min}$. Dynamic mechanical properties of constructs were also determined in the wet state after seeding and culturing of chondrocytes. 


\section{Cell Culture}

Bovine chondrocytes were isolated as previously reported and allowed to proliferate in the presence of expansion medium consisting of Dulbecco's modified Eagle's medium (DMEM, Invitrogen) supplemented with 10\% heat-inactivated fetal bovine serum (FBS, Sigma-Aldrich), $0.2 \mathrm{mM}$ ascorbic acid 2-phosphate (Invitrogen), $0.1 \mathrm{mM}$ nonessential amino acids (Sigma-Aldrich), $10 \mathrm{mM}$ HEPES (Invitrogen), $0.4 \mathrm{mM}$ proline (Sigma-Aldrich), $100 \mathrm{U} / \mathrm{ml}$ penicillin and $100 \mu \mathrm{g} / \mathrm{ml}$ streptomycin (both Invitrogen). ${ }^{[2]}$

Before cell seeding, the PTMC scaffolds were sterilized in ethanol for $30 \mathrm{~min}$, washed 3 times with sterile phosphate-buffered saline (PBS, Gibco-BRL), placed in 96-well plates (1 scaffold/well) and incubated overnight in DMEM. After cell expansion, the cells were detached from the culture flasks with trypsin and the PTMC scaffolds were subsequently seeded by adding $100 \mu$ l expansion medium containing 250000 cells on top of each scaffold. The culture plates containing the constructs were placed overnight on an orbital shaker in an incubator at $37{ }^{\circ} \mathrm{C}$ in humidified air with $5 \% \mathrm{CO}_{2}$. The next day, each well was supplemented with $100 \mu \mathrm{l}$ differentiation medium consisting of DMEM containing $100 \mu \mathrm{g} / \mathrm{ml}$ sodium pyruvate (Sigma), $0.2 \mathrm{mM} \mathrm{L-glutamine,} 50 \mathrm{mg} / \mathrm{ml}$ insulin-transferrinselenite (ITS+Premix, BD Sciences), $10 \mathrm{ng} / \mathrm{ml}$ transforming growth factor $\beta-3$ (TGF- $\beta 3$, R\&D Systems), $0.1 \mu \mathrm{M}$ dexamethasone (Sigma), $100 \mathrm{U} / \mathrm{ml}$ penicillin and $100 \mu \mathrm{g} / \mathrm{ml}$ streptomycin. ${ }^{[2]}$ Subsequently, culture media were changed every 2 days for a period up to 6 weeks by adding $200 \mu \mathrm{l}$ fresh differentiation medium per well. Constructs were collected after 3 and 6 weeks of culturing and subjected to microscopical, histological and mechanical analyses.

\section{Microscopical and Histological Analyses}

After culturing for 3 and 6 weeks PTMC constructs were analyzed by SEM. Specimens were fixed overnight in $0.14 \mathrm{M}$ cacodylate buffer ( $\mathrm{pH}$ 7.2-7.4) containing 1.5\% glutaraldehyde (Merck) followed by dehydration by immersion in sequential ethanol series. Subsequently, the specimens were critical-point-dried from liquid carbon dioxide using a Balzers CPD 030 machine (Bal-Tec, Balzers, Switzerland). Samples were then sputtercoated with gold and analyzed by SEM as described above.

PTMC scaffolds with and without cells were also collected after 3 and 6 weeks for histological analysis. The samples were washed with PBS and fixed in a $10 \%$ buffered 
formalin solution for $1 \mathrm{~h}$. Subsequently, the specimens were dehydrated in sequential ethanol series, embedded in Cryomatrix (Shandon), and cut at $-18{ }^{\circ} \mathrm{C}$ into $5 \mu \mathrm{m}$ sections using a cryotome (77210160 GB, Shandon). The sections were stained with $1 \%$ Alcian Blue (Sigma-Aldrich) and 1\% Picrosirius Red (Polysciences) to visualize sulphated glycosaminoglycans and fibrillar collagens, respectively. The applied staining protocols were performed according to the manufacturer's instructions.

Mounted slides stained with Alcian Blue were examined under a light microscope (Eclipse E400, Nikon, Japan) and samples stained with Picrosirius Red were examined under the same microscope with a polarized lens. Representative images were captured with a digital camera (Sony, Japan).

\section{Results and Discussion}

\section{Characterization of PTMC Network Films}

A three-armed methacrylated PTMC macromer with a molecular weight of $3100 \mathrm{~g} / \mathrm{mol}$ was synthesized in a two-step reaction using trimethylene carbonate, glycerol and methacrylic anhydride. The monomer to oligomer conversion and the oligomer to macromer conversion were 95 and $79 \%$, respectively, as determined by ${ }^{1} \mathrm{H}-\mathrm{NMR}$. However, as no peaks corresponding to unreacted oligomer chain ends can be discerned upon functionalization, it can be assumed that functionalization was essentially complete. ${ }^{[12]}$ The PTMC macromer was formulated into a photo-crosslinkable resin having a viscosity of approximately $10 \mathrm{~Pa} \cdot \mathrm{s}$, applying propylene carbonate as non-reactive diluent.

First, flat PTMC network films with a thickness of $482 \pm 16 \mu \mathrm{m}$, after extraction and drying, were characterized. The volume degree of swelling in chloroform $(5.3 \pm 0.2)$ was somewhat higher than that of a network of the same macromer, photo-crosslinked in the melt without non-reactive diluent. ${ }^{[11]}$ This indicates that formulation of the resin with the diluent results in a lower crosslinking density of the PTMC network films. Networks formed with extended polymer chains due to the presence of the diluent, exhibit fewer entanglements and more elastically inactive network chains due to intra-molecular reactions, giving supercontracted network chains after deswelling and drying. ${ }^{[20]}$ However, the PTMC network films prepared with the diluent-containing resin, still had a high degree of crosslinking as indicated by the relatively high midpoint glass transition temperature of 
$-8.8^{\circ} \mathrm{C}$. Networks prepared with macromers with higher molecular weights, and thus lower crosslinking densities, show lower midpoint glass transition temperatures of approximately $-15{ }^{\circ} \mathrm{C} .{ }^{[11]}$ In agreement with the effects of the non-reactive diluent discussed above, the PTMC network films formed in the diluted state had a somewhat lower E-modulus $(3.7 \pm 0.4 \mathrm{MPa})$ and maximum tensile strength $(1.7 \pm 0.1 \mathrm{MPa})$, a comparable toughness $\left(76 \pm 7 \mathrm{~N} / \mathrm{mm}^{2}\right)$ and a considerably higher elongation at break $(85 \pm 4 \%)$ as compared to network films prepared by photo-crosslinking in the melt. ${ }^{[1]}$

With a water uptake of $1.0 \pm 0.3 \%$, the PTMC network films are quite hydrophobic. This complies with the static water contact angle of $93 \pm 2^{\circ}$, measured on the dry network surface. However, conditioning in water leads to a lower static contact angle of $61 \pm 3^{\circ}$. This decrease is most probably caused by reorientation or diffusion of polymer end groups at the polymer-water interface due to the uptake of small amounts of water. ${ }^{[21]}$

\section{Characterization of the PTMC scaffold}

The designed scaffold was built from the PTMC resin in the stereolithography apparatus, applying a layer thickness of $25 \mu \mathrm{m}$. After extraction and drying, the scaffolds had shrunk $18.3 \pm 0.6 \%$ to a diameter of $4.87 \pm 0.03 \mathrm{~mm}$ and a height of $6.29 \pm 0.03 \mathrm{~mm}$. Figure 2 shows the gyroid pore architecture and pore interconnectivity of the built PTMC structure by $\mu \mathrm{CT}$ and SEM. As determined by $\mu \mathrm{CT}$ analysis, the scaffolds had an average pore size of $350 \pm 12 \mu \mathrm{m}$ and a porosity of $54.0 \pm 2.2 \%$, with $84 \%$ of the pores having diameters in the range of 300 to $415 \mu \mathrm{m}$ (see also Figure 4 below). This well-defined scaffold architecture allows seeding, proliferation and migration of cells. Moreover, the transport of nutrients and metabolic waste products will be facilitated by the high interconnectivity of the pores. ${ }^{[22]}$ 

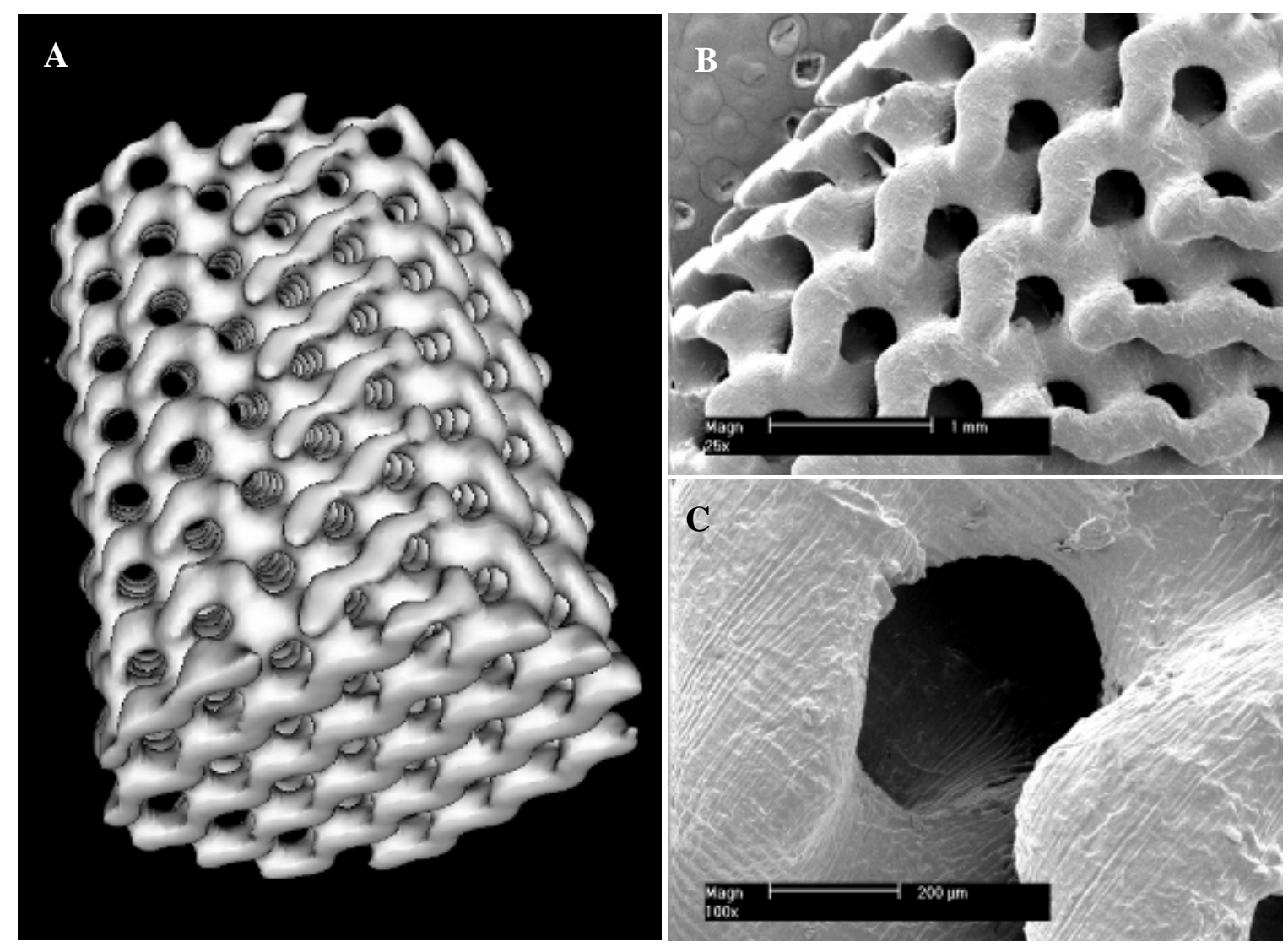

Figure 2. Visualization of scaffolds built from the PTMC resin by stereolithography. $\mu C T \operatorname{scan}(A)$ and SEM (B and $C)$.

\section{Culturing of Chondrocytes in PTMC Scaffolds}

To evaluate the behavior of chondrocytes in the designed porous PTMC structures, 250000 cells were seeded per scaffold and subsequently cultured under stationary conditions up to 6 weeks. As shown in Figure 3A, cells adhered to the PTMC scaffold after 3 weeks. The surface was almost completely covered with spread cells. After 6 weeks (Figure 3B), the porous structure was fully confluent with cells. Moreover, at this time point also cells with a round morphology were present on top of the spread cells. In cartilage tissue engineering, chondrocytes should preferentially adopt a round shape since these are the differentiated cells producing cartilage-specific proteoglycans and collagen fibrils with a high ratio of collagen type II over type I. ${ }^{[23]}$ The same cell behavior as shown in Figures 3A and 3B has been reported by Mahmood et al. using ceramic substrates. ${ }^{[24]}$ Chondrocytes were found to adhere and spread on the surface of the ceramic, whereas cells remained their round shape when they were attached to spread cells. Moreover, cell morphology was dependent on the cell seeding density, with an increase in the number of 
round and aggregated cells at a higher seeding density. Likewise, the number of round and presumably differentiated chondrocytes in our scaffold could be improved by increasing the seeding density of the cells.

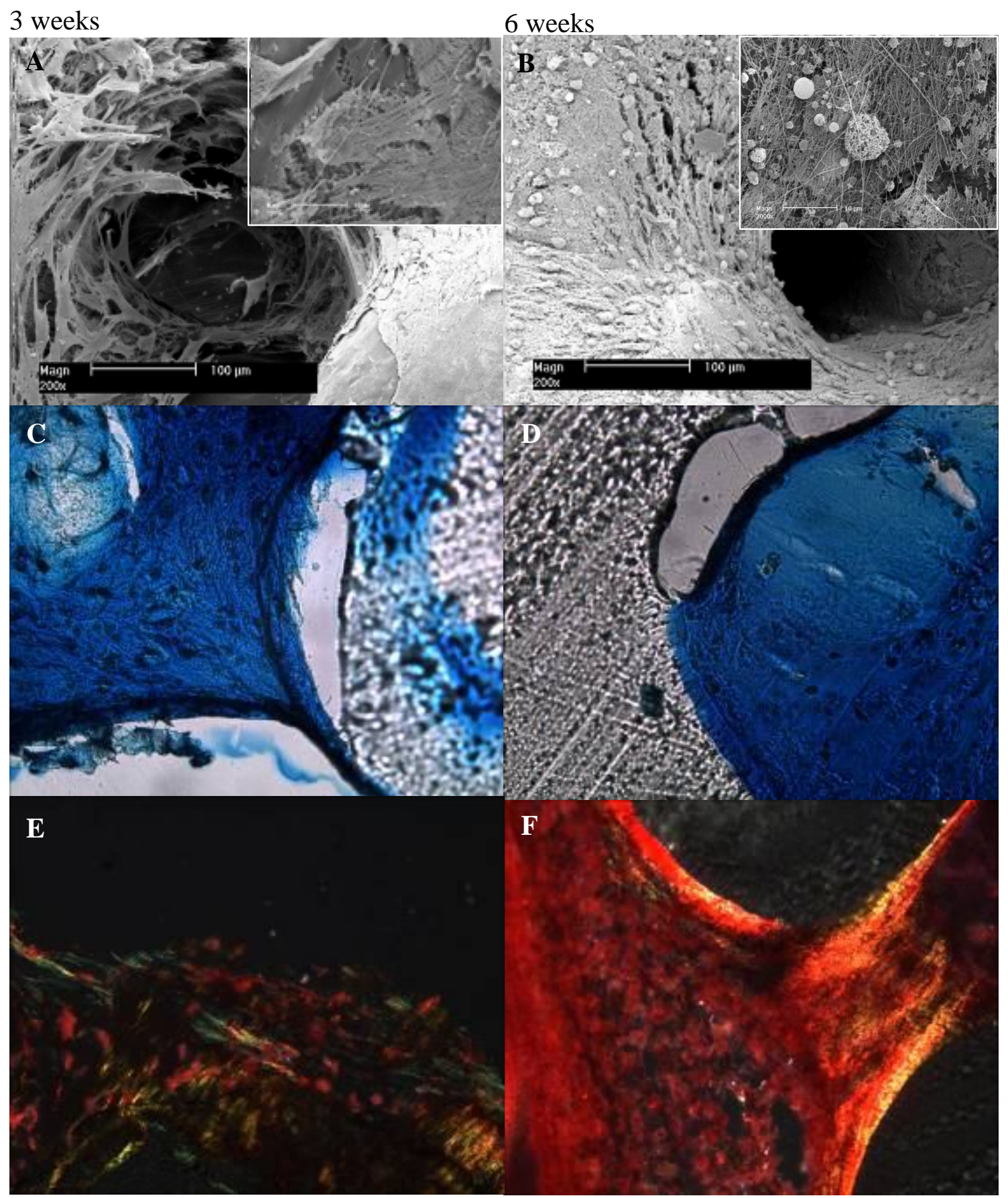

Figure 3. SEM (A,B), Alcian blue staining $(C, D)$ and Picrosirius red staining $(E, F)$ of constructs after culturing for 3 weeks $(A, C, E)$ and 6 weeks $(B, D, F)$. 
Alcian blue staining of cross-sections of PTMC constructs showed that the chondrocytes synthesized sulphated glycosaminoglycans after culturing for 3 and 6 weeks (Figures 3C and 3D, respectively). Sulphated glycosaminoglycans are main components of cartilagespecific proteoglycans, indicating appropriate matrix production by the cells. This is further substantiated by Picrosirius red staining for fibrillar collagens as shown in Figures $3 \mathrm{E}$ and 3F. Thin and loosely packed collagen fibrils stain green to yellow whereas thick and tightly packed fibrils stain yellow to red. Although this staining protocol does not allow distinguishing collagen type II indicative of cartilaginous matrix, the cross-sections clearly show that fibrillar collagen is produced by the cells. Presumably, these fibrils are also visible in the inset of Figure 3B. Alcian blue and Picrosirius red did not stain the PTMC material (data not shown).

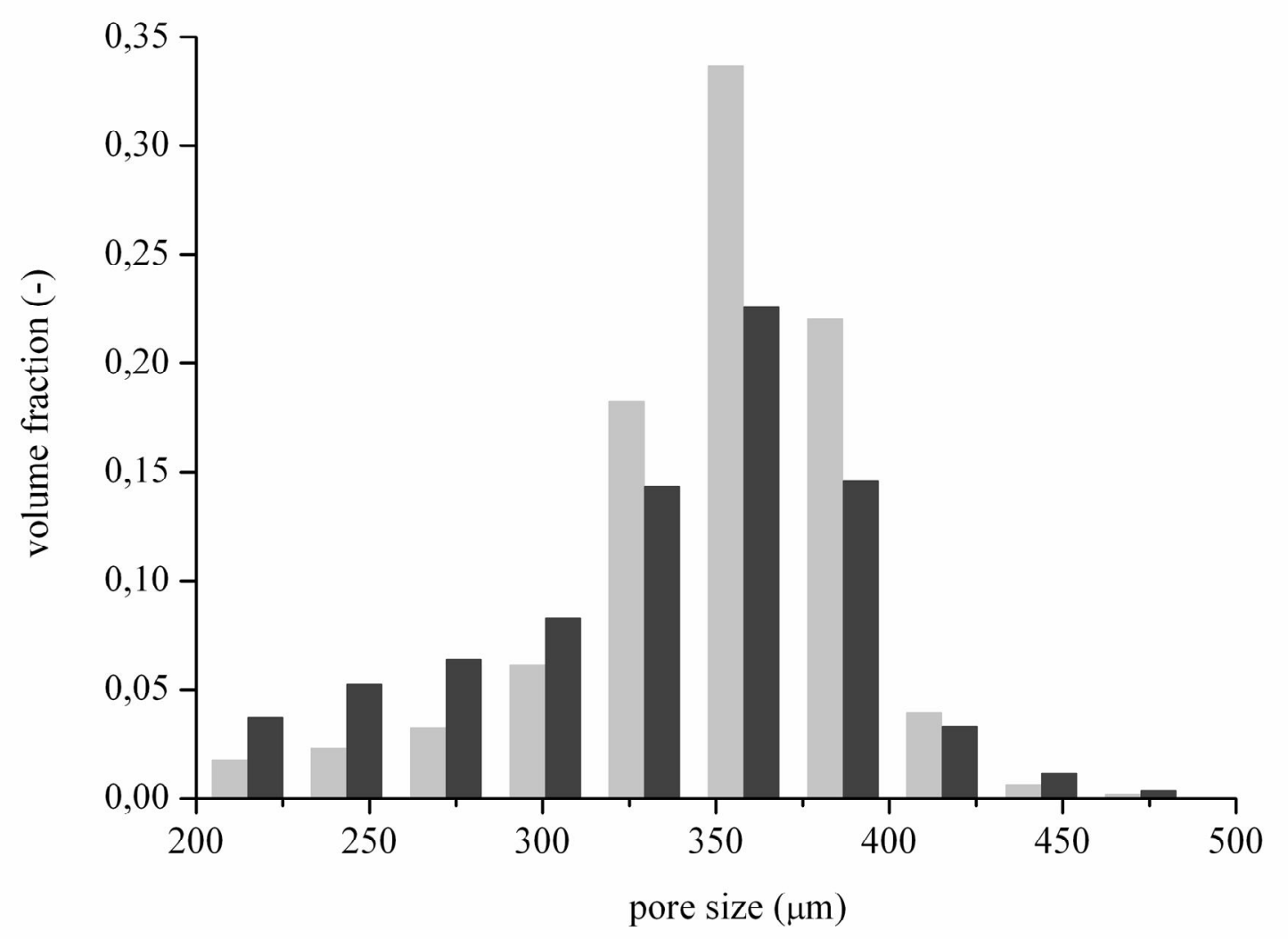

Figure 4. Pore size distributions determined by $\mu C T$ analysis of PTMC scaffolds without cells (light grey bars) and PTMC constructs after culturing for 6 weeks (dark grey bars).

The chondrocyte-seeded scaffolds were also investigated by $\mu \mathrm{CT}$, showing that after a culture period of 6 weeks the constructs were still porous. The average pore size and 
porosity were found to be decreased from $350 \pm 12 \mu \mathrm{m}$ to $325 \pm 19 \mu \mathrm{m}$ and from $54.0 \pm 2.2 \%$ to $39.2 \pm 9.3 \%$, respectively (Figure 4). This indicates that after 6 weeks the pores were not yet completely filled with cells and extracellular matrix. It should be noted, however, that degradation of the PTMC might have concomitantly increased the porosity of the constructs.

It has been reported that the hydrophilicity of scaffolds for cartilage tissue engineering is a key determinant for re-differentiation of seeded chondrocytes and preservation of the differentiated phenotype. ${ }^{[25-27]}$ In these studies, porous poly(ethylene glycol)-terephthalate poly(butylene terephthalate) (PEGT/PBT) scaffolds were used, of which the hydrophilicity could be increased by using poly(ethylene glycol) (PEG) with a higher molecular weight and by increasing the weight percentage of PEGT in the copolymer. It was shown that chondrocytes seeded in hydrophilic scaffolds adopted a round morphology and expressed a high ratio of collagen type II over type I. This was correlated with a low adsorption of fibronectin to the substrates, resulting in little adhesion and spreading of chondrocytes on the surface of the scaffolds. Static water contact angles of flat films of the material were $39 \pm 1^{\circ}$. Although the PTMC network films used in our study became more hydrophilic upon conditioning in water, the static water contact angle of $61 \pm 3^{\circ}$ is most probably too high to promote the differentiated chondrocyte phenotype. Photo-crosslinking of copolymers or blends of PTMC and PEG by stereolithography may be an alternative approach to prepare more hydrophilic scaffolds for cartilage tissue engineering. Yet another approach may be to use a chondrocyte-containing hydrogel to seed the cells in the designed scaffolds. Chondrocytes suspended in suitable hydrogels, e.g. mimicking the cartilaginous extracellular matrix, maintain the round chondrocyte morphology or re-differentiate to this phenotype. $^{[2,23]}$

\section{Mechanical Properties of PTMC Scaffolds and Constructs}

To characterize the mechanical properties of scaffolds without and with cells, dynamic compression tests were performed at different temperatures. Table 1 shows the compression moduli of scaffolds without cells, tested in dry and wet state, and of constructs with chondrocytes analyzed in the wet state after 3 and 6 weeks of culture.

Comparing scaffolds in the dry and wet state showed that the compression moduli decreased due to water uptake and subsequent swelling of the network. The decrease was more pronounced at $21^{\circ} \mathrm{C}$ than at $37^{\circ} \mathrm{C}$ (Table 1). Dynamic mechanical analysis further 
showed that the compression moduli increased approximately 50\% upon culturing of the scaffolds with chondrocytes for 6 weeks. Upon improving the approach presented here in terms of promotion of the differentiated phenotype of the cells (e.g. higher seeding densities, more hydrophilic scaffolds) and further maturation of the constructs (e.g. dynamic culturing, mechanical stimulation), the moduli may approach the values of natural cartilage tissue which range from $450-800 \mathrm{kPa}$ at room temperature. ${ }^{[28]}$

Table 1. Compression moduli of PTMC scaffolds and constructs determined in the dry and wet state. Values are given as average \pm standard deviation of 5 samples.

\begin{tabular}{|c|c|c|c|c|}
\hline & \multirow{3}{*}{$\begin{array}{l}\text { state of } \\
\text { scaffold }\end{array}$} & \multirow{3}{*}{$\frac{\text { culture period }}{\text { weeks }}$} & \multirow{2}{*}{\multicolumn{2}{|c|}{$\begin{array}{c}\text { compression modulus } \\
\mathbf{k P a} \\
\end{array}$}} \\
\hline & & & & \\
\hline & & & $\mathbf{T}=21^{\circ} \mathrm{C}$ & $\mathbf{T}=37^{\circ} \mathrm{C}$ \\
\hline \multirow{2}{*}{ without cells } & dry & - & $119 \pm 14$ & $60 \pm 7$ \\
\hline & wet & - & $69 \pm 15$ & $47 \pm 13$ \\
\hline \multirow{2}{*}{ with cells } & wet $^{\mathrm{a}}$ & 3 & 76 & 46 \\
\hline & wet & 6 & $102 \pm 28$ & $68 \pm 22$ \\
\hline
\end{tabular}

${ }^{\mathrm{a}}$ Values obtained from single measurement.

\section{Conclusions}

A three-armed methacrylated PTMC macromer with a molecular weight of $3100 \mathrm{~g} / \mathrm{mol}$ was formulated into a photo-crosslinkable resin, using propylene carbonate as non-reactive diluent. By photo-crosslinking of the resin, tough, flexible and relatively hydrophobic network films were prepared.

The resin was used to build designed scaffolds with a gyroid pore architecture by means of stereolithography. Highly interconnected porous structures were prepared with an average pore diameter of $350 \pm 12 \mu \mathrm{m}$ and a porosity of $54.0 \pm 2.2 \%$. Bovine chondrocytes were seeded in the scaffolds and were found to adhere and spread on the PTMC surface. After culturing for 6 weeks, the porous structures were completely covered with cells. Also cells with a round morphology were present, indicative of the differentiated chondrocyte phenotype, adhering to the spread cells. Sulphated glycosaminoglycans and fibrillar 
collagens were deposited by the cells. During culturing for 6 weeks, the compression moduli of the constructs increased $50 \%$ to approximately $100 \mathrm{kPa}$. Optimization of chondrocyte re-differentiation upon seeding will further increase the applicability of these constructs for cartilage tissue engineering.

\section{References}

[1] Calderon L, Collin E, Velasco-Bavon D, Murphy M, O'Halloran D, Pandit A. Type II collagen-hyaluronan hydrogel - a step towards a scaffold for intervertebral disc tissue engineering. Eur Cell Mater 2010, 20, 134-148.

[2] Jin R, Teixeira LSM, Dijkstra PJ, van Blitterswijk CA, Karperien M, Feijen J. Enzymatically-crosslinked injectable hydrogels based on biomimetic dextranhyaluronic acid conjugates for cartilage tissue engineering. Biomaterials 2010, 31, 3103-3113.

[3] Scotti C, Mangiavini L, Boschetti F, Vitari F, Domeneghini C, Fraschini G, Peretti GM. Effect of in vitro culture on a chondrocyte-fibrin glue hydrogel for cartilage repair. Knee Surg Sport Tr A 2010, 18, 1400-1406.

[4] Chan-Park MB, Zhu AP, Shen JY, Fan AL. Novel photopolymerizable biodegradable triblock polymers for tissue engineering scaffolds: synthesis and characterization. Macromol Biosci 2004, 4, 665-673.

[5] Dhariwala B, Hunt E, Boland T. Rapid prototyping of tissue-engineering constructs, using photopolymerizable hydrogels and stereolithography. Tissue Eng 2004, 10, 1316-1322.

[6] Ifkovits JL, Burdick JA. Review: photopolymerizable and degradable biomaterials for tissue engineering applications. Tissue Eng 2007, 13, 2369-2385.

[7] Sachlos, E. and J.T. Czernuszka. Making tissue engineering scaffolds work. Review: the application of solid freeform fabrication technology to the production of tissue engineering scaffolds. Eur Cell Mater 2003, 5, 29-40.

[8] Peltola SM, Melchels FPW, Grijpma DW, Kellomaki M. A review of rapid prototyping techniques for tissue engineering purposes. Ann Med 2008, 40, 268-280.

[9] Hollister SJ. Porous scaffold design for tissue engineering. Nat Mater 2005, 4, 518524.

[10] Melchels FPW, Feijen J, Grijpma DW. A review on stereolithography and its applications in biomedical engineering. Biomaterials 2010, 31, 6121-6130.

[11] Chapter 5 of this thesis.

[12] Chapter 3 of this thesis.

[13] Chapter 4 of this thesis.

[14] Chapter 7 of this thesis.

[15] Chapanian R, Tse MY, Pang SC, Amsden BG. The role of oxidation and enzymatic hydrolysis on the in vivo degradation of trimethylene carbonate based photocrosslinkable elastomers. Biomaterials 2009, 30, 295-306.

[16] Melchels FPW, Feijen J, Grijpma DW. A poly(D,L-lactide) resin for the preparation of tissue engineering scaffolds by stereolithography. Biomaterials 2009, 30, 38013809.

[17] EnvisionTEC Machine Data Sheet "Perfectory ${ }^{\circledR}$ SXGA $^{+}$W/ERM Mini Multi Lens" 2008. 
[18] Jansen J, Melchels FPW, Grijpma DW, Feijen J. Fumaric acid monoethyl esterfunctionalized poly(D,L-lactide)/N-vinyl-2-pyrrolidone resins for the preparation of tissue engineering scaffolds by stereolithography. Biomacromolecules 2009, 10, 214220.

[19] Hildebrand T, Rüegsegger P. A new method for the model-independent assessment of thickness in three-dimensional images. Journal of Microscopy-Oxford 1997, 185, 6775.

[20] Premachandra JK, Mark JE. Effects of dilution during crosslinking on strain-induced crystallization in cis-1,4-polyisoprene networks. I. Experimental results. J Macromol Sci-Pure Appl Chem A 2002, 39, 287-300.

[21] Bat E, Plantinga JA, Harmsen MC, van Luyn MJA, Zhang Z, Grijpma DW, Feijen J. Trimethylene carbonate and epsilon-caprolactone based (co)polymer networks: mechanical properties and enzymatic degradation. Biomacromolecules 2008, 9, 32083215.

[22] Melchels FPW, Barradas AM, van Blitterswijk CA, de Boer J, Feijen J, Grijpma DW. Effects of the architecture of tissue engineering scaffolds on cell seeding and culturing. Acta Biomater 2010, 6, 4208-4217.

[23] Jin R, Moreira Teixeira LS, Krouwels A, Dijkstra PJ, van Blitterswijk CA, Karperien M, Feijen J. Synthesis and characterization of hyaluronic acid-poly(ethylene glycol) hydrogels via Michael addition: An injectable biomaterial for cartilage repair. Acta Biomater 2010, 6, 1968-1977.

[24] Mahmood TA. Chondrocyte-biomaterial interactions: A mechanistic approach to cartilage tissue engineering. PhD Thesis, University of Twente, 2003, Chapter 6.

[25] Woodfield TB, Miot S, Martin I, van Blitterswijk CA, Riesle J. The regulation of expanded human nasal chondrocyte re-differentiation capacity by substrate composition and gas plasma surface modification. Biomaterials 2006, 27, 1043-1053.

[26] Miot S, Woodfield TB, Daniels AU, Suetterlin R, Peterschmitt I, Heberer M, van Blitterswijk CA, Riesle J, Martin I. Effects of scaffold composition and architecture on human nasal chondrocyte re-differentiation and cartilaginous matrix deposition. Biomaterials 2005, 26, 2479-2489.

[27] Mahmood TA, Miot S, Frank O, Martin I, Riesle J, Langer R, van Blitterswijk CA. Modulation of chondrocyte phenotype for tissue engineering by designing the biologic-polymer carrier interface. Biomacromolecules 2006, 7, 3012-3018.

[28] Mansour JM. Biomechanics of cartilage. www.cartilagehealth.com, accessed 01 April 2011. 


\title{
Chapter 7
}

\section{Preparation of a Designed Poly(Trimethylene Carbonate) Microvascular Network by Stereolithography}

\begin{abstract}
A photo-crosslinkable resin based on a poly(trimethylene carbonate) (PTMC) macromer with a molecular weight of $3150 \mathrm{~g} / \mathrm{mol}$ was used in stereolithography to prepare designed flexible and elastic network structures. The physical properties of the networks and the compatibility of built structures with human umbilical vein endothelial cells (HUVECs) were evaluated. The hydrophobic networks were found to be flexible and elastic, with an $\mathrm{E}$ modulus of $7.9 \pm 0.1 \mathrm{MPa}$, a tensile strength of $3.5 \pm 0.1 \mathrm{MPa}$ and an elongation at break of $76.7 \pm 0.7 \%$. HUVECs attached and proliferated well on the surfaces of the built structures. A three-dimensional microvascular network was designed to serve as a perfusable scaffold for tissue engineering. In the design, 5 generations of open channels branch into 4 smaller channels yielding a microvascular region with a high density of capillaries. The overall cross-sectional area through which medium or blood can be perfused remains constant. These structures would ensure efficient nourishment of cells in a large volume of tissue.

Microvascular network structures were built by stereolithography using the PTMC resin. The smallest channels had square cross-sectional areas, with inner widths of approximately $224 \mu \mathrm{m}$ and wall thicknesses of approximately $152 \mu \mathrm{m}$. The channels were open, allowing water to perfuse the scaffold at $0.279 \pm 0.006 \mathrm{ml} / \mathrm{s}$ at $80 \mathrm{mmHg}$ and $0.335 \pm 0.009 \mathrm{ml} / \mathrm{s}$ at $120 \mathrm{mmHg}$.
\end{abstract}




\section{Introduction}

In the United States the number of patients waiting for a donor organ was 110783 in April 2011, while only 2406 organ transplantations were performed in January 2011. ${ }^{[1]}$ As the number of patients waiting for a donor organ by far exceeds the number of available organs, there is an urgent need for organs. Use of the available artificial organs has drawbacks, but an alternative does not yet exist. Tissue engineering may change this situation in the future. Unfortunately, the engineering of vital organs and complex tissues is limited by the lack of an intrinsic blood supply, which leads to cell death when the thickness of the tissue exceeds 1-2 mm. ${ }^{[2]}$ A three-dimensional microvascular network for the distribution of oxygen and nutrients to the cells, and the removal of metabolic waste products from the cells concurrently needs to be engineered.

Microfabrication techniques have been used to prepare such microvascular systems. ${ }^{[2,3]}$ Using non-degradable poly(dimethyl siloxane) (PDMS) and biodegradable polymers like polylactic acid (PLA), polyglycolic acid (PGA), poly(lactic-co-glycolic) acid (PLGA), polyglycerol sebacate (PGS) and polyurethanes, two-dimensional vascular structures were made. ${ }^{[2,4-8]}$ To create three-dimensional constructs, up to 35 layers of these were stacked. ${ }^{[9]}$ This method, however, is cumbersome and poorly reproducible.

With stereolithography, designed three-dimensional structures can reproducibly be prepared at high resolution. This solid free-form fabrication technique is an additive fabrication process with which structures are built in a layer-by-layer manner by photo-crosslinking. In each layer, a (pixel) pattern is defined that spatially controls the curing and solidification of a liquid resin by light. Illuminating resin layers with different patterns allows the fabrication of a designed three dimensional construct, as each layer adheres to the preceding one.

For medical applications, resins based on poly(propylene fumarate) (PPF), poly(ethylene glycol) (PEG), poly(D,L-lactide) (PDLLA) and trimethylene carbonate (TMC) (co)polymers have been formulated and used in stereolithography. ${ }^{[10-18]}$ Recently, we prepared designed flexible and elastic porous structures for use in (soft) tissue engineering by stereolithography using poly(trimethylene carbonate) (PTMC)-based resins. ${ }^{[19-20]}$

In this work we describe the preparation and characterization of a flexible threedimensional biodegradable microvascular network by stereolithography using a PTMCbased photo-crosslinkable resin. The design of this microvasculature is considerably more advanced than that of previous (stacked) two dimensional structures. ${ }^{[2-3,21]}$ For the 
successful engineering of complex tissues and organs, the intricate vascular network design should be precisely reproduced in the stereolithography process.

\section{Materials and Methods}

\section{Materials}

Polymerization grade 1,3-trimethylene carbonate (1,3-dioxan-2-one, TMC) was obtained from Boehringer Ingelheim (Germany). Glycerol (99.5+\% spectrophotometric grade) was purchased from Aldrich (USA) and dehydrated by briefly heating at $180{ }^{\circ} \mathrm{C}$. Stannous octoate (stannous 2-ethylhexanoate, $\mathrm{SnOct}_{2}$ ) was used as received from Sigma (USA). Methacrylic anhydride (94\%) and hydroquinone were purchased from Aldrich (Germany), and ( \pm )- $\alpha$-Tocopherol (vitamin E) from Fluka (Switzerland). Lucirin TPO-L (ethyl 2,4,6trimethylbenzoylphenyl phosphinate) was provided by BASF (Germany). Orasol Orange G was a gift from Ciba Special Chemicals (Switzerland). Technical grade ethanol was purchased from Chempopack (Belgium), and propylene carbonate from Merck (Germany). Serva silicone solution to silanize the glassware was obtained from SERVA Electrophoresis GmbH (Germany).

\section{Poly(Trimethylene Carbonate) Macromer and Resin}

Poly(trimethylene carbonate) (PTMC) macromer was synthesized in a two step reaction. ${ }^{\text {[24] }}$ First, a three-armed hydroxyl-terminated poly(trimethylene carbonate) (PTMC) oligomer was prepared by ring opening polymerization (oligomerization) of TMC using glycerol as initiator and $\operatorname{SnOct}_{2}(0.07 \mathrm{wt}-\%)$ as catalyst. To obtain an oligomer with a molecular weight of approximately $3000 \mathrm{~g} / \mathrm{mol}, 1.3 \mathrm{~mol} \mathrm{TMC}, 4.4 \times 10^{-2}$ mol glycerol and $2.2 \times 10^{-4} \mathrm{~mol}$ SnOct $_{2}$ were charged into a freshly silanized, dried three-necked flask and polymerized in an argon atomosphere at $130{ }^{\circ} \mathrm{C}$ for 3 days. Then, the hydroxyl-terminated oligomer was functionalized by reaction with $19.8 \times 10^{-2}$ mol methacrylic anhydride (an excess of $50 \mathrm{~mol} \%$ ) at $120{ }^{\circ} \mathrm{C}$ for 3 days. To avoid premature crosslinking, $0.06 \mathrm{wt} \%$ hydroquinone and $0.06 \mathrm{wt} \%( \pm)$ - $\alpha$-tocopherol (both relative to the amount of oligomer) were used. The macromer was purified by removal of the formed methacrylic acid and the excess methacrylic anhydride by vacuum distillation at $85{ }^{\circ} \mathrm{C}$. 
The TMC monomer conversion in the oligomerization reaction, the degree of functionalization with methacrylic end-groups and the molecular weight of the obtained macromers were determined by ${ }^{1} \mathrm{H}-\mathrm{NMR}$ spectroscopy (Varian Innova $300 \mathrm{MHz}$, USA), using $\mathrm{CDCl}_{3}$ (Merck, Germany) as solvent.

A photo-crosslinkable resin was prepared at a temperature of $80{ }^{\circ} \mathrm{C}$ by mixing the PTMC macromer with 4.75 wt\% Lucirin TPO-L and 0.15 wt\% Orasol Orange G dye when required. The viscosity of the resin was determined at temperatures between 40 and $60{ }^{\circ} \mathrm{C}$ at shear rates of $0.28,1.86$ and 3.72 1/s, using a Brookfield DV-E rotating spindle viscometer, equipped with a small sample adapter and a Brookfield s21 spindle which rotated at respectively $0.3,2$ and $4 \mathrm{rpm}$.

\section{Stereolithography}

An EnvisionTec Perfactory Mini Multilens stereolithography apparatus was used to build flat solid films (for the evaluation of the physical properties of the photo-crosslinked networks) and designed three-dimensional disks and microvascular networks. The apparatus is equipped with a digital micro-mirror device, ${ }^{[22]}$ allowing the projection of $1280 \times 1024$ pixels measuring $32 \times 32 \mu \mathrm{m}^{2}$. The wavelength of the (blue) light with which the PTMC resin is crosslinked ranged from 400 and $550 \mathrm{~nm}$, and had a maximum at $440 \mathrm{~nm} .^{[15]}$ With use of an electrical heating element, the resin could be photo-crosslinked at temperatures varying from room temperature to approximately $60{ }^{\circ} \mathrm{C}$. To precisely control the depth of cure when building the constructs, stereolithography working curves were first prepared to assess the curing behaviour of the resin. ${ }^{[15]}$

The solid films were prepared by crosslinking single layers of PTMC resin with thicknesses of 0.15 and $0.55 \mathrm{~mm}$ at a light intensity of $10 \mathrm{~mW} / \mathrm{cm}^{2}$. (In this case the resin did not contain Orasol Orange dye.) Rectangular areas of $75 \times 60 \mathrm{~mm}^{2}$ were irradiated for $5 \mathrm{~min}$, the resulting network films were post-cured in a nitrogen atmosphere in a UV crosslinking cabinet at $365 \mathrm{~nm}$ for $30 \mathrm{~min}$ (Ultralum, USA, UV light intensity is $3-4 \mathrm{~mW} / \mathrm{cm}^{2}$ ). The films were extracted with 1:1 mixtures of propylene carbonate and ethanol for one week, refreshing the solvent mixtures twice a day. During the following week, the films were deswollen by daily replacing the solvent mixtures with mixtures containing increasing amounts of ethanol. The films were then washed with ethanol and dried under vacuum until constant weight. 
Designed three-dimensional microvascular networks were prepared in the stereolithography apparatus by photo-crosslinking (light intensity of $17 \mathrm{~mW} / \mathrm{cm}^{2}$ ) subsequent layers of resin with thicknesses of $25 \mu \mathrm{m}$ upon which a pixel pattern is projected. For the assessment of the compatibility of the PTMC networks with cells, upright standing disks with a diameter of $5.5 \mathrm{~mm}$ and a thickness of $1.0 \mathrm{~mm}$ were also designed and directly prepared by stereolithography. In these cases the resins contained the macromer, the photoinitiator as well as the dye.

The structures and the disks were designed using a computer-aided design software package (Rhinoceros v4, McNeel, Europe), and converted into STL files for use in the stereolithography apparatus. ${ }^{[23]}$

The microvascular networks and disks were extracted with 1:2 mixtures of propylene carbonate and ethanol. For one week, the solvent mixtures were refreshed three times a day. Very careful de-swelling of the built structures was done during another week by daily refreshing the mixtures with solvent mixtures to which increasing amounts of ethanol were added. The structures were then washed with ethanol and dried under vacuum until constant weight.

\section{Physical Properties of Photo-Crosslinked PTMC Resins}

Thicker PTMC films obtained by photo-crosslinking the PTMC resin in the stereolithography apparatus were used in determining the network characteristics, thermal and mechanical properties, hydrophilicity and water uptake behaviour of the PTMC networks.

Disks with a diameter of $8 \mathrm{~mm}$ were punched out of the extracted PTMC network films and swollen in chloroform for $45 \mathrm{hrs}$. The degree of swelling (q) at room temperature was determined in triplicate according to equation 1:

$$
q=1+\frac{m_{\text {swollen }}-m_{d r y}}{m_{d r y}} \times \frac{\rho_{P T M C}}{\rho_{C H C l_{3}}}
$$

In this equation $m_{s w o l l e n}$ is the mass of the solvent swollen specimen and $m_{d r y}$ is the mass of the extracted specimen in the dry state. The densities of chloroform $\left(\rho_{\mathrm{CHCl}_{3}}\right)$ and PTMC $\left(\rho_{\text {PTMC }}\right)$ are respectively $1.48 \mathrm{~g} / \mathrm{cm}^{3}$ and $1.31 \mathrm{~g} / \mathrm{cm}^{3}$.

The glass transition temperatures $\left(\mathrm{T}_{\mathrm{g}}\right)$ of the extracted PTMC networks were determined by differential scanning calorimetry (DSC), using a Perkin Elmer Pyris 1 (USA). A sample of 
approximately $10 \mathrm{mg}$ was heated from $-100{ }^{\circ} \mathrm{C}$ to $100{ }^{\circ} \mathrm{C}$ at a heating rate of $10{ }^{\circ} \mathrm{C} / \mathrm{min}$ and quenched at $300{ }^{\circ} \mathrm{C} / \mathrm{min}$ to $-100{ }^{\circ} \mathrm{C}$. After $5 \mathrm{~min}$, a second scan was recorded from which onset- and midpoint values of the glass transition temperature were determined. Temperature calibration was done using cyclohexane, indium and lead calibration standards.

The tensile properties of the photo-crosslinked PTMC network were determined at room temperature using a Zwick Z020 tensile tester (Germany) equipped with a $500 \mathrm{~N}$ load cell according to ASTM D882-91. The specimens measured $72 \times 5.5 \mathrm{~mm}^{2}$, the initial grip-to-grip separation was $50 \mathrm{~mm}$ and the cross-head speed was $50 \mathrm{~mm} / \mathrm{min}$. The elongation of the specimens was derived from the distance between the grips. The Young's modulus, maximum tensile strength, elongation at break and toughness of the networks were determined in duplicate as previously described. ${ }^{[24]}$

The hydrophilicity and water uptake of photo-crosslinked PTMC was evaluated using disks with a diameter of $8 \mathrm{~mm}$ diameter which were punched out of the extracted films. The disks were allowed to equilibrate in ultra-pure water (MilliQ Plus-Millipore, France) for $45 \mathrm{~h}$ at room temperature, after which the water uptake was calculated according to equation 2:

$$
\text { water uptake }=\frac{m_{w e t}-m_{d r y}}{m_{d r y}} \times 100
$$

where $m_{w e t}$ is the mass of the water-swollen network and $m_{d r y}$ the mass of the initial dry specimen. Static-, advancing- and receding contact angles of ultra-pure water on the dry extracted network surface were determined using a video-based OCA 20 contact angle measuring system from DataPhysics (Germany), equipped with an electronic syringe module. The measurements were performed at room temperature on five different regions of the film; the presented values are given as average \pm standard deviation.

The permeation of oxygen $\left(\mathrm{O}_{2}\right)$ through the photo-crosslinked PTMC films was evaluated at a feed pressure of 1.5 bar and a temperature of $37{ }^{\circ} \mathrm{C}$, using the setup described elsewhere. ${ }^{[25]}$ The gas permeability coefficient $(P)$ [Barrer] was obtained from the gas permeance $(P / l)\left[10^{-6} \mathrm{~cm}^{3} /\left(\mathrm{cm}^{2} \cdot \mathrm{s} \cdot \mathrm{cmHg}\right)\right]$ and the thicknesses $(l)[\mathrm{cm}]$ of the films. The gas permeance is determined from the steady-state pressure increase in time at the permeate side in a calibrated volume, assuming the ideal gas law to be valid. ${ }^{[26]}$ This is done using equation 3: 


$$
\frac{P}{l}=\frac{V \times 273.15 \times\left(p_{p t}-p_{p 0}\right)}{A \times T \times \frac{1}{2} \times\left(p_{f t}-p_{f 0}\right) \times 76 \times t} \times 10^{6}
$$

Here, $p_{p t}$ and $p_{f t}[\mathrm{bar}]$ are respectively the gas pressure at the permeate- and at the feed side at time $t$ [s], while $p_{p 0}$ and $p_{f 0}$ are respectively the gas pressure of the permeate and feed at $t=0$. In this equation, $T[\mathrm{~K}]$ is the temperature, $V\left[\mathrm{~cm}^{3}\right]$ the calibrated permeate volume and $A\left[\mathrm{~cm}^{2}\right]$ the area of the film through which exchange takes place. Experiments were conducted in duplicate; the results are expressed as an average value \pm standard deviation.

\section{Compatibility of Photo-Crosslinked PTMC Resins with Cells}

Human umbilical vein endothelial cells (HUVECs) were cultured in a humidified atmosphere of $5 \% \mathrm{CO}_{2}$ in air at $37{ }^{\circ} \mathrm{C}$, in $300 \mathrm{~cm}^{2}$ flasks containing endothelial cell medium (EGM2). The medium was refreshed twice a week until cells reached confluence. Upon confluence, the cells were trypsinized and counted.

After extraction and drying, the PTMC disks prepared by stereolithography were disinfected with $70 \%$ ethanol for 3 hours and left to dry overnight in a laminar flow hood. Afterwards, the films were extensively washed with PBS and placed in non-treated 96-well plates (Greiner, Germany, 14mg/well, surface area: approximately $0.35 \mathrm{~cm}^{2} /$ well). The use of these plates prevents cell adhesion to the bottom of the wells. The cells were seeded on the disks at a density of $10^{4}$ cells and incubated at $37^{\circ} \mathrm{C}$ for $4 \mathrm{~h}$ to allow cell attachment. Then, fresh medium was added until a final volume of $200 \mu$ per well was reached. The HUVECs were cultured for $4 \mathrm{~h}$, and 1, 3, 7 and 14 days. Control culturing experiments were done on tissue culture polystyrene under the same conditions. At each time point, cell attachment, proliferation, distribution and morphology were assessed microscopically after staining with methylene blue.

\section{Characterization of PTMC Microvascular Networks Built by Stereolithography}

The designed microvascular networks that were built by stereolithography and subsequently extracted and dried, were imaged and visualized at a spatial resolution of $14 \mu \mathrm{m}$ by micro-computed tomography $(\mu \mathrm{CT})$ using a GE eXplore Locus SP scanner. Scanning electron microscopy (SEM) using a Philips XL30, The Netherlands, operating at $2 \mathrm{kV}$ was employed as well. 


\section{Results and Discussion}

\section{Photo-Crosslinkable PTMC Resins}

A three-armed PTMC macromer was synthesized by ring opening polymerization of trimethylene carbonate using glycerol as the initiator, and subsequently functionalized with methacrylic anhydride. Analyses by ${ }^{1} \mathrm{H}-\mathrm{NMR}$ showed that the monomer conversion in the ring opening polymerization was $99 \%$, and that the degree of functionalization with methacrylate groups was $90 \%$. The number average molecular weight of the macromer was $3150 \mathrm{~g} / \mathrm{mol}$. The macromer has a glass transition temperature of $-33^{\circ} \mathrm{C}$, and is a colourless, very viscous liquid at room temperature.

To be able to process PTMC resins in stereolithography, much lower viscosities are required. Although a diluent can also be used to reduce viscosity, we found that the diluentswollen network structures that form upon crosslinking were very fragile and failures in the stereolithography process very often occurred. Much better results were obtained when building the structures at elevated temperatures using undiluted resin compositions. (The resins could also be formulated without use of a diluent by mixing the macromer and other compounds at higher temperatures.) In the investigated temperature range of 40 to $60{ }^{\circ} \mathrm{C}$, the resin containing macromer and Lucirin TPO-L photo-initiator behaved in a Newtonian manner, and its viscosity decreased from $149.5 \mathrm{~Pa} \cdot \mathrm{s}$ at $40{ }^{\circ} \mathrm{C}$ and $21.4 \mathrm{~Pa} \cdot \mathrm{s}$ at $50{ }^{\circ} \mathrm{C}$ to $7.3 \mathrm{~Pa} \cdot \mathrm{s}$ at $60{ }^{\circ} \mathrm{C}$.

\section{Properties of PTMC Networks Prepared by Stereolithography}

To evaluate the physical properties of PTMC network structures prepared by stereolithography, solid network films were first prepared in the stereolithography apparatus at a temperature of $60{ }^{\circ} \mathrm{C}$. A layer of resin (not containing dye) with a thickness of $0.55 \mathrm{~mm}$ was crosslinked by illumination, and then post-cured, extracted, de-swollen and dried as described. The thicknesses of the final PTMC network films were $0.49 \pm 0.02 \mathrm{~mm}$. To assess their network characteristics, the crosslinked films were swollen in chloroform. At room temperature, their volume degrees of swelling were 4.1 \pm 0.1 . As the network films had been post-cured in a second step and had been extracted, de-swollen and cured, their gel contents were not determined. 
The films were flexible, transparent and amorphous with a broad glass transition at low temperature. The onset value of the glass transition ( $\mathrm{T}_{\mathrm{g}}$ (onset)) was determined to be $-13.2{ }^{\circ} \mathrm{C}$, while the midpoint value $\left(\mathrm{T}_{\mathrm{g}}\right.$ (midpoint)) was $-7.8^{\circ} \mathrm{C}$. In tensile testing, the value of the elasticity modulus was determined to be $7.9 \pm 0.1 \mathrm{MPa}$, while the maximum tensile strength and elongation at break were respectively $3.5 \pm 0.1 \mathrm{MPa}$ and $76.7 \pm 0.7 \%$. The toughness of the networks, as determined from the area under the curves in the stress-strain diagrams, was $123.8 \pm 2.8 \mathrm{~N} / \mathrm{mm}^{2}$. This is in agreement with previous work. ${ }^{[24]}$

Water uptake experiments showed that the photo-crosslinked PTMC network films were quite hydrophobic, with an equilibrium water uptake at room temperature of $1.02 \pm 0.18 \%$. The water contact angles of the films determined after immediately placing a droplet of water on the films were $84.8 \pm 5.6^{\circ}, 87.8 \pm 3.8^{\circ}$ and $53.45 \pm 6.90^{\circ}$ respectively for the static, advancing and receding contact angle. Comparable values were found for PTMC networks that were formed by irradiation of linear high molecular weight PTMC with UV light. ${ }^{\text {[27] }}$

\section{Three-Dimensional PTMC Network Structures Prepared by Stereolithography}

Compared to the preparation of sheets and films by stereolithography, the preparation of designed (porous) three-dimensional structures is much more involved. To be able to accurately build such structures at high resolutions, it is necessary to be able to precisely control the depth of cure in the resin layers that are consecutively photo-crosslinked in the process.

To convert a liquid resin into a (semi-) solid state, a minimum amount of energy is required. This is the critical energy $\left(E_{c}\right.$ in $\left.J / \mathrm{m}^{2}\right)$. The curing characteristics of a resin under given experimental conditions can be evaluated by recording a stereolithography working curve. The working curve describes the relationship between the thickness of the cured layer (cure depth $\mathrm{C}_{\mathrm{d}}$ in $\mathrm{m}$ ) and the light irradiation dose $\left(\mathrm{E}\right.$ in $\left.\mathrm{J} / \mathrm{m}^{2}\right)$. For each resin and resin formulation the working curve is unique. The cure depth can be influenced by adjusting the light penetration depth $\left(D_{p}\right.$ in $\left.m\right)$ and by varying the light irradiation dose $E$.

The working curve is expressed as shown in equation $4:^{[28]}$

$$
C_{d}=D_{p} \times \ln \frac{E}{E_{c}}
$$

The light irradiation dose $\mathrm{E}$ is determined by the intensity of the light used in photo-crosslinking and the duration of time ( $\mathrm{t}$ in $\mathrm{s}$ ) to which the resin is exposed to. 
As result of absorption, addition of a dye to the resin decreases the light penetration depth and significantly increases the accuracy with which the depth of cure can be controlled. ${ }^{[29]}$

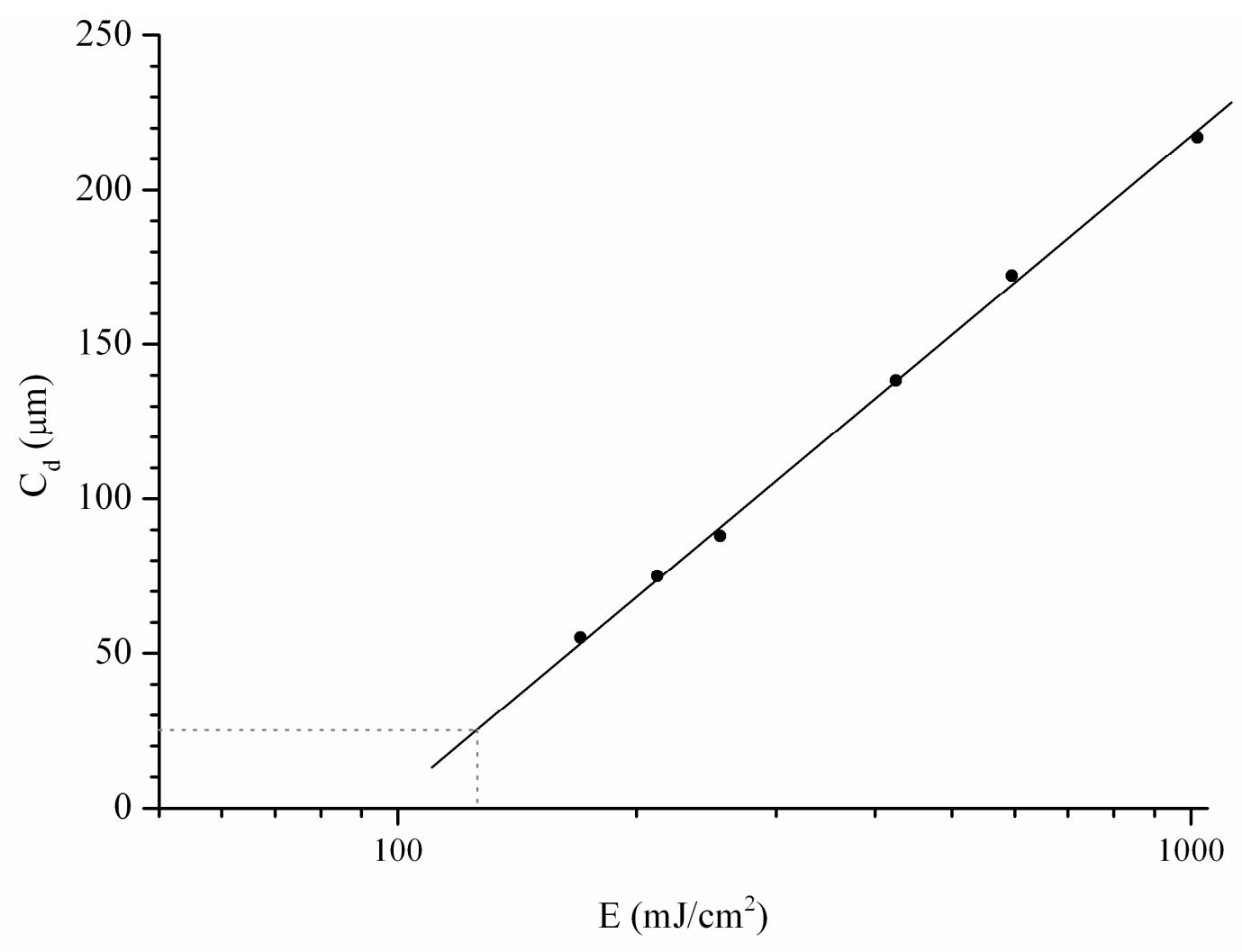

Figure 1. Stereolithography working curve of the PTMC resin at $60{ }^{\circ} \mathrm{C}$. The resin contained macromer with a number average molecular weight of $3150 \mathrm{~g} / \mathrm{mol}, 4.75 \mathrm{wt} \%$ Lucirin TPO-L and 0.15 wt\% Orasol Orange $G$ dye. Note the logarithmic scale of the $x$-axis.

In Figure 1 the working curve at $60{ }^{\circ} \mathrm{C}$ for the resin containing the PTMC macromer, $4.75 \mathrm{wt} \%$ photo-initiator and $0.15 \mathrm{wt} \%$ dye is presented. The relationship between the depth of cure under these conditions and the irradiation dose was determined in triplicate by illuminating the resin for different exposure times of up to $60 \mathrm{~s}$. A resin layer thickness of $25 \mu \mathrm{m}$ was used to prepare the three dimensional structures at high resolution. The working curve shows that to reach this cure depth $124.1 \mathrm{~mJ} / \mathrm{cm}^{2}$ is required. At a light intensity of $17 \mathrm{~mW} / \mathrm{cm}^{2}$, this corresponds to an exposure time of $7.30 \mathrm{~s}$. To ensure bonding between the sequentially photo-crosslinked layers a somewhat longer exposure time is required. However, too long exposure times will lead to significant deviations of the built structures when compared to the design. This is especially relevant when building porous structures. 
We found that an overcure of $100 \%$ resulted in optimal results, and prepared our structures by illuminating each layer of resin for $14.6 \mathrm{~s}$.

Solid structures in the form of disks were built to investigate the compatibility of the photocrosslinked PTMC networks with cells. Human umbilical vein endothelial cells (HUVECs) were cultured for 14 days on the disks built by stereolithography. Although initially the networks were quite hydrophobic (static water contact angle $\sim 85^{\circ}$, see before) equilibration in water increases their hydrophilicity, and static water contact angles of $61 \pm 3^{\circ}$ are now measured. Cells readily adhered to the surfaces of the PTMC networks, Figure 2A and 2D show HUVECs stained with methylene blue on the surface of the disks 4 hrs after seeding. Although at this time point the cells appeared to be round, it was found that they significantly proliferated and showed a stretched morphology after 1 day of culture. See Figure $2 \mathrm{~B}$ and $2 \mathrm{E}$. After 3 days the cells formed a near confluent layer over the entire surface of the disks. The confluent cell layer remained stable in culture for at least 14 days.
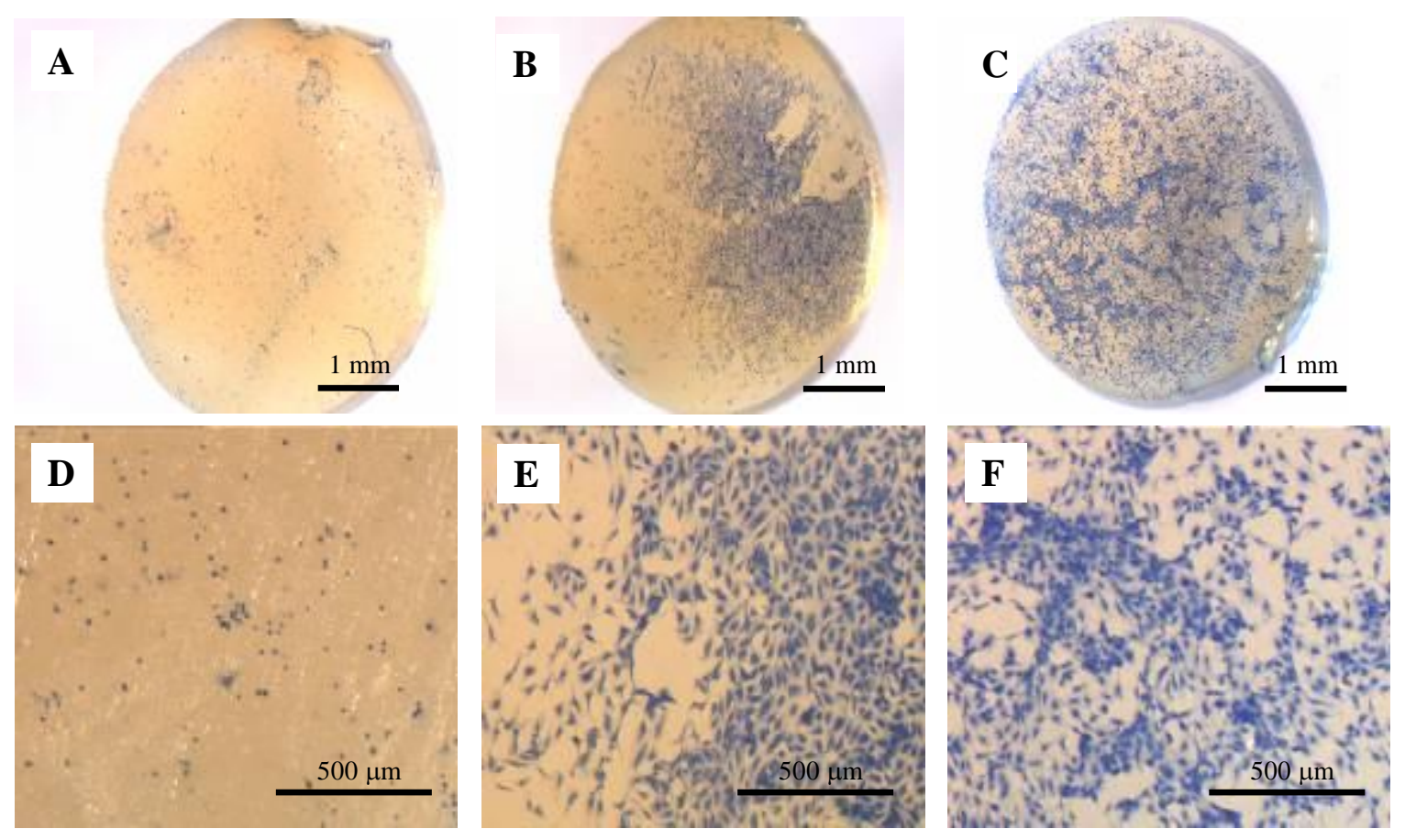

Figure 2. Light microscopy images of adhering HUVECs stained with methylene blue on the surface of PTMC network disks prepared by stereolithography. Images were taken $4 \mathrm{~h}$ after seeding ( $A$ and $D)$, after 1 day ( $B$ and $E$ ) and after 3 days ( $C$ and $F$ ) of culturing. 


\section{Designed Microvascular Network Structures Prepared by Stereolithography}

The appearance and structural organization of the microvasculature can differ in different organs and tissues, and it can change its structural and functional characteristics in response to stimuli. ${ }^{[30-31]}$ The complexity of a vasculature system is illustrated in Figure 2A, where a SEM image of a casting of the temporal lobe vasculature of a chinchilla is shown.

In designing a (human) vasculature, the following hierarchy applies and should be taken into account: arteries with diameters of 1-2 mm divide into a network of arteriolae with diameters of $100-1000 \mu \mathrm{m}$. These divide into smaller meta-arteriole branches with diameters of 80-100 $\mu \mathrm{m}$, which finally become capillaries with diameters of 10-15 $\mu \mathrm{m}$. The arterial vessel system distributes blood through the body, and as the diameters of the branching structures decrease in the tissues, pressure is reduced. This allows most efficient exchange of metabolites. Each cell is in close proximity to a capillary (within a distance of 150-200 $\mu \mathrm{m}$ ). These eventually combine into a venous collecting system that removes metabolic waste products, which also serves as a capacitance system. ${ }^{[32-34]}$
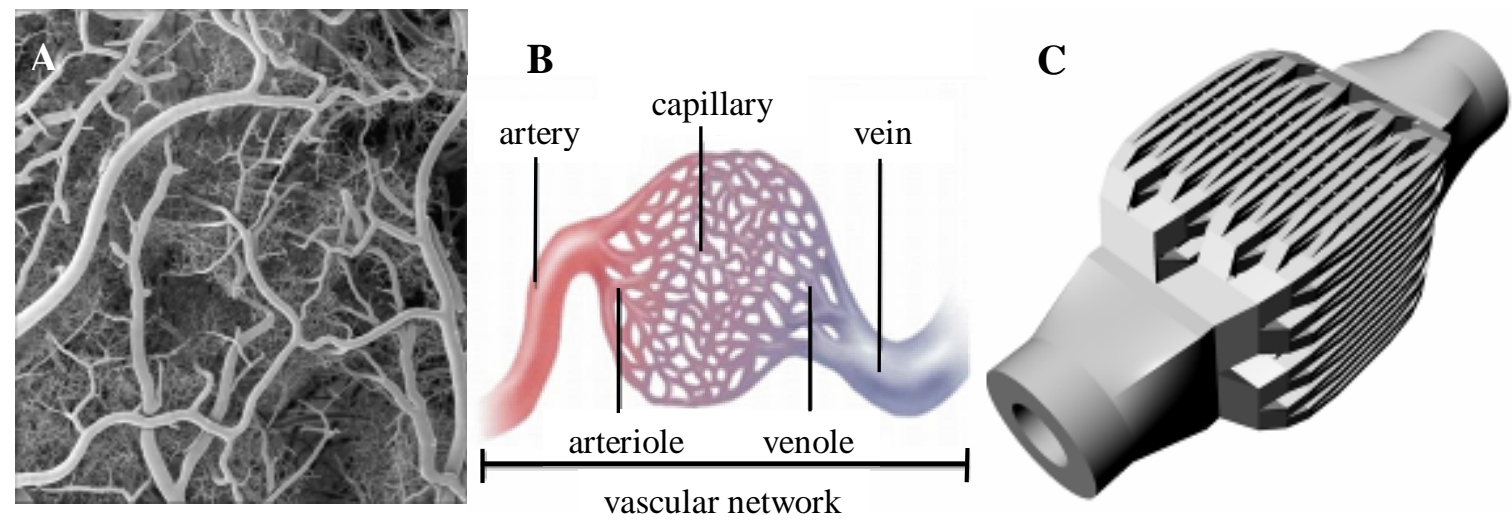

Figure 3. The vascular system: an arterial vessel system branches into a microvascular (capillary) network, which reunites into a venous vessel system. SEM image of a casting of a chinchilla temporal lobe vasculature (A, taken from reference 35). Schematic textbook illustration of the vascular system (B, adapted from reference 42). Simplified three-dimensional design used to prepare a microvascular network structure for use as a scaffold in tissue engineering by stereolithography $(C)$.

Schematic representations, such as the one shown in Figure 3B, served as a starting point in the design of a three-dimensional model of a vascular system with a high density of capillaries. This design is presented in Figure 3C. When used as a tissue engineering 
scaffold, such a branching network of hollow vessels could ensure the supply of oxygen and nutrients to the cells being cultured and the removal of waste products from them. As in our stereolithography setup a pattern of pixels measuring $32 \times 32 \mu \mathrm{m}^{2}$ is projected on the resin, hollow capillary channels with square cross sections were designed. Such design will allow the fabrication of open microvascular structures with the smallest possible features. With each generation, the hollow channels split into four channels with equal inner crosssectional area. The ends of the structure were tubular to allow connection of the perfusable scaffolds in a bioreactor or to be anastomosed with the host vasculature upon implantation. In addition, minimally-sized supporting struts were incorporated into the design to stabilize the structure during the building process.

In initial experiments it was determined that the designed microvascular network structures could be built using the PTMC-based resin when the wall thicknesses of the smallest capillaries were larger than approximately $150 \mu \mathrm{m}$. When wall thicknesses were smaller, the structures were too fragile to be built and ruptured halfway during the process.

In Figure 4 the features of an extracted microvascular network built according to the design of Figure 3C using the PTMC macromer resin that was formulated with photo-initiator and dye are shown. Directly after the building process, the structure was $38.9 \mathrm{~mm}$ high and $15.6 \mathrm{~mm}$ wide. Figure 4A shows a photograph of the microvascular network after extraction, de-swelling and drying. The network was found to be very flexible and elastic. It had a height of $34.8 \mathrm{~mm}$ and a width of $14.1 \mathrm{~mm}$; this shrinkage of $7.8 \pm 1.6 \%$ is likely not only due to the removal of non-reacted macromer, but also due to cooling from the building temperature of $60{ }^{\circ} \mathrm{C}$ to room temperature.

An image obtained by reconstruction of the data of a $\mu \mathrm{CT}$ scan is shown in Figure 4B. The image demonstrates that the smallest channels of the built microvascular network are well-separated and hollow. The scanning electron microscopy images of cross-sections of the built structures presented in Figures 4C and 4D confirm that the smallest channels are hollow. In addition the higher resolution of the method in comparison to $\mu \mathrm{CT}$ illustrates the layer-by-layer nature of the stereolithographic manufacturing process.

Scanning electron microscopy images of the extracted microvascular networks such as those shown in Figure 4D allow determination of the dimensions of the branched channels of the different generations. In Table 1, the dimensions of the walls and the open cross-sectional area are compared with those designed. 

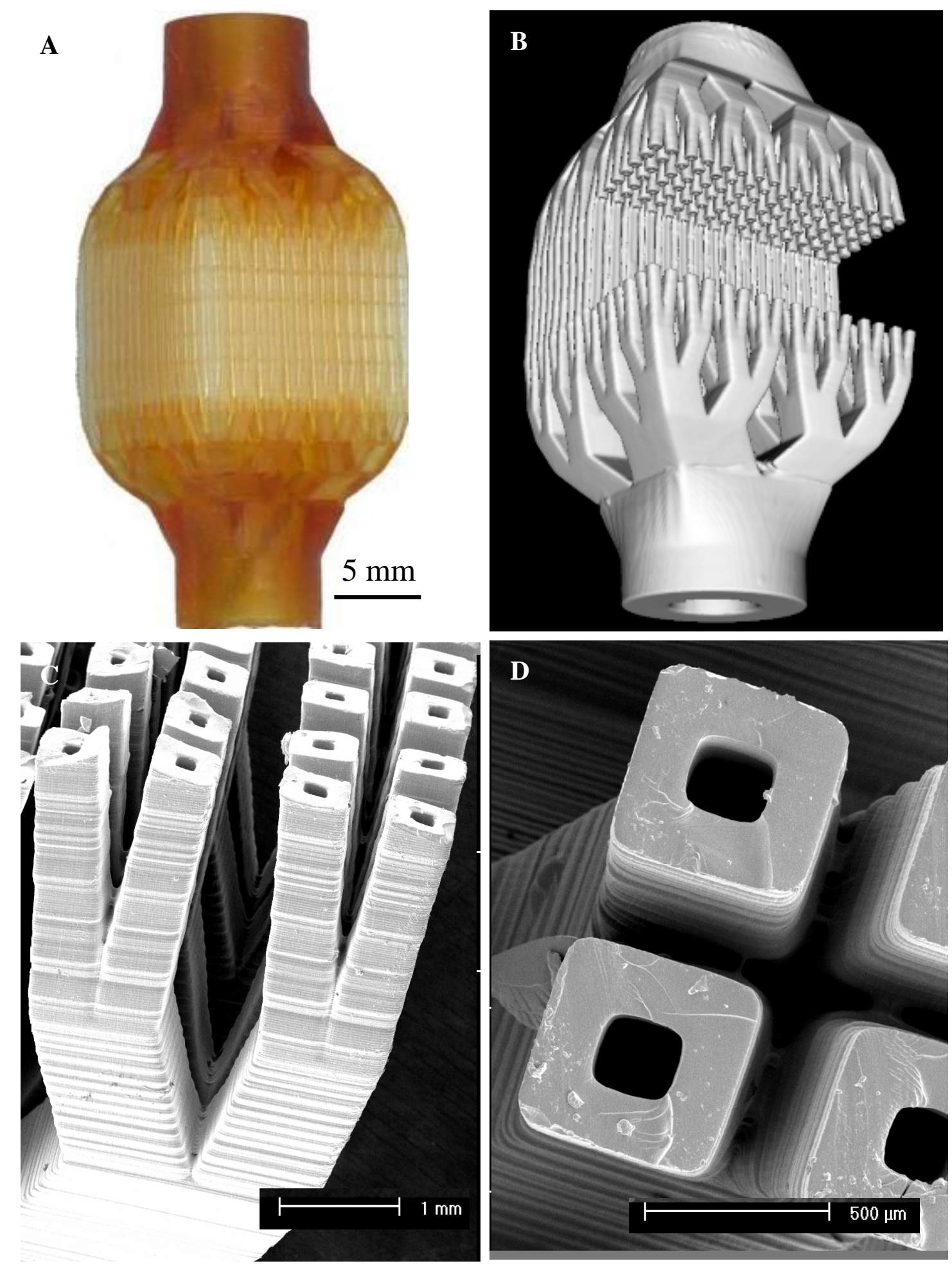

Figure 4. Designed three-dimensional microvascular network for use as a perfusable tissue engineering scaffold built by stereolithography using a PTMC-based resin. The design is shown in Figure 3C. Photograph (A) and reconstructed $\mu C T$ image (B) of the microvascular network. SEM images of cross-sections of the microvascular structure showing that the channels with the smallest diameters are fully open ( $C$ and $D)$. 
Table 1 shows that the characteristics of the channels are well reproduced in the built microvascular network. They are very well retained after extraction, de-swelling and drying of the crosslinked network. The inner diameter of the channel and its wall thickness decreases with each branching step. However, as each generation splits into 4 separate branches, the cross-sectional area through which a liquid can be perfused remains open and only decreases from 20 to $13 \mathrm{~mm}^{2}$. Although as a whole the built structure has shrunk after extraction and drying, the wall thickness of the smallest channels is somewhat higher than designed. This can be the result of additional thermal polymerization of the resin due to the exothermic nature of the photo-crosslinking reaction. ${ }^{[36]}$

Table 1. Channel dimensions of the different generations of the microvascular network prepared according to the design of Figure 3C by stereolithography using a PTMC-based resin. The dimensions of the built microvascular networks were determined after extraction, de-swelling and drying.

\begin{tabular}{|c|c|c|c|c|c|c|}
\hline \multirow{4}{*}{$\begin{array}{l}\text { channel } \\
\text { generation }\end{array}$} & \multirow{4}{*}{$\begin{array}{c}\text { total } \\
\text { number of } \\
\text { channels } \\
\text { per } \\
\text { cross- } \\
\text { section }\end{array}$} & \multicolumn{2}{|c|}{ wall thickness } & \multicolumn{3}{|c|}{ open cross-sectional area } \\
\hline & & \multirow{2}{*}{ designed } & \multirow{2}{*}{ obtained } & \multirow{2}{*}{$\begin{array}{c}\text { designed } \\
\text { per } \\
\text { channel }\end{array}$} & \multicolumn{2}{|c|}{ obtained } \\
\hline & & & & & per channel & total \\
\hline & & $\mathbf{m m}$ & $\mathbf{m m}$ & $\mathbf{m m}^{2}$ & $\mathbf{m m}^{2}$ & $\mathbf{m m}^{2}$ \\
\hline 1 & 1 & 2.288 & $2.275 \pm 0.054$ & 20.94 & $19.97 \pm 1.50$ & $19.97 \pm 1.50$ \\
\hline 2 & 4 & 1.144 & $0.982 \pm 0.042$ & 5.23 & $3.47 \pm 0.09$ & $13.88 \pm 0.38$ \\
\hline 3 & 16 & 0.572 & $0.572 \pm 0.093$ & 1.31 & $0.97 \pm 0.09$ & $15.58 \pm 1.44$ \\
\hline 4 & 64 & 0.286 & $0.267 \pm 0.005$ & 0.33 & $0.22 \pm 0.03$ & $14.39 \pm 1.68$ \\
\hline 5 & 256 & 0.143 & $0.152 \pm 0.006$ & 0.08 & $0.05 \pm 0.01$ & $12.95 \pm 1.66$ \\
\hline
\end{tabular}

Water was perfused through the branched capillary network structures to simulate the flow of medium or blood in a tissue engineering cell culturing experiment. Typical blood pressures of healthy persons are 120 over $80 \mathrm{mmHg}$. The microvascular structures were tested to pressures of $140 \mathrm{mmHg}$ which they could easily withstand. Using pre-wetted structures, water flow rates were determined to be $0.279 \pm 0.006 \mathrm{ml} / \mathrm{s}$ at $80 \mathrm{mmHg}$ and $0.335 \pm 0.006 \mathrm{ml} / \mathrm{s}$ at $120 \mathrm{mmHg}$. These values correspond to flow velocities of respectively 
18.6 \pm 2.9 and $22.3 \pm 3.5 \mathrm{ml} /\left(\mathrm{s} \cdot \mathrm{mm}^{2}\right)$. In the arteriolar limb of capillaries at rest, for example, a blood flow velocity of $0.84 \pm 0.53 \mathrm{ml} /\left(\mathrm{s} \cdot \mathrm{mm}^{2}\right)$ has been measured. ${ }^{[37]}$

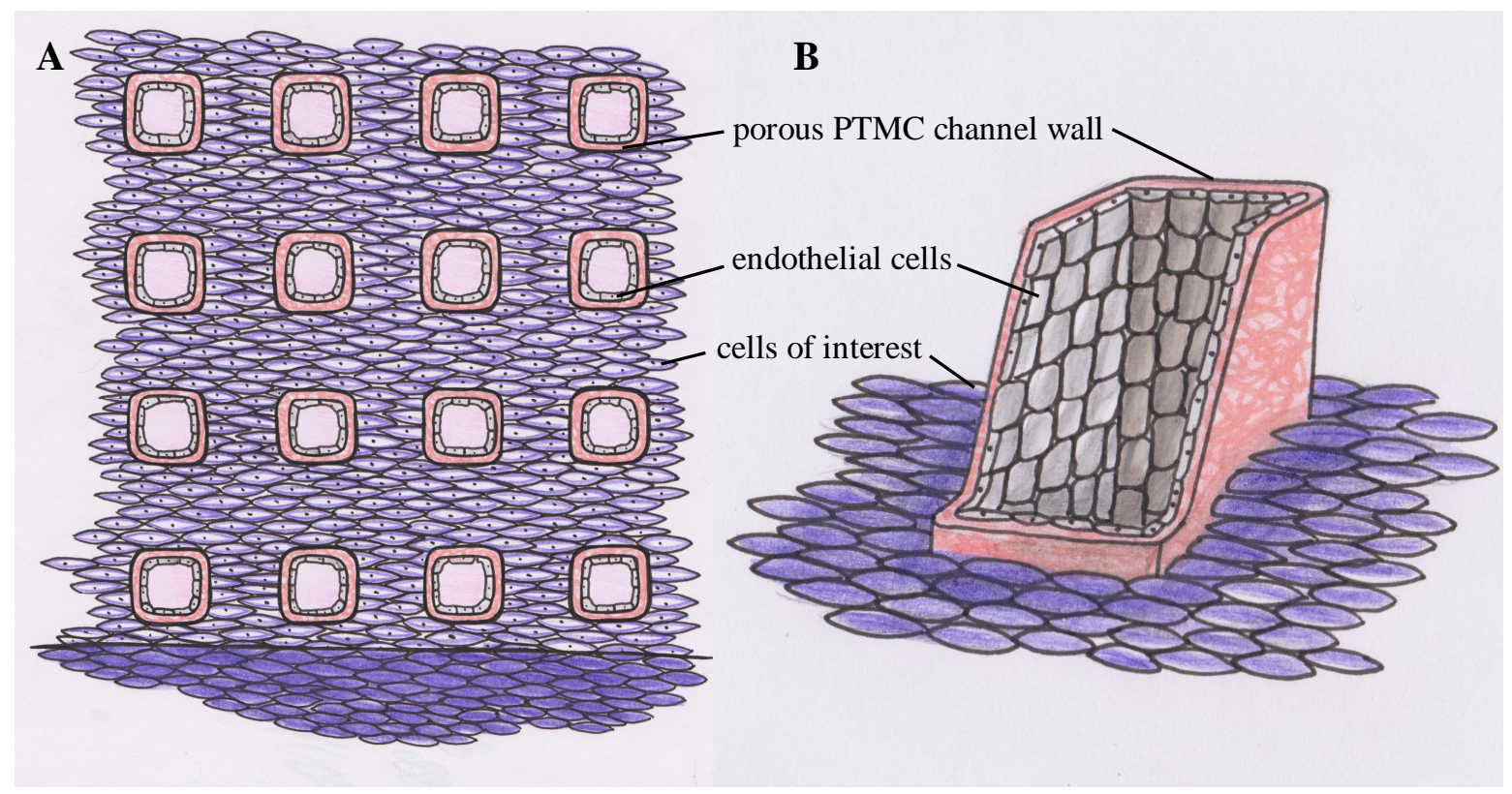

Figure 5. Scheme of the cross-section (A) of an engineered tissue prepared using a microvascular network scaffold built by stereolithography. The magnification of a microvascular channel $(B)$ shows that the lumen of the porous channels is coated with endothelial cells. Porous channel walls provide cells of interest, cultured on the outside of the channels, with nutrients and oxygen, allowing them to proliferate and built up native tissue.

For a cell to survive and for an organ to function properly, adequate concentrations of oxygen are required. In healthy tissue the supply of oxygen, as mentioned before, is ensured by the microcirculation. ${ }^{[38]}$ It is clear that to adequately function as a microvascular network and scaffold in tissue engineering, oxygen and nutrients also need to reach the cells in the developing tissues. This implies that the walls of the capillary channels in our structures need to be permeable to these compounds. Figure 5 shows a scheme of a cross-section of an engineered tissue containing open microvascular channels with porous walls. The lumen of the microvascular channels of the scaffold are coated with endothelial cells, while the cells of interest were seeded on the outside of the channels where they proliferated as they can now be reached by nutrients present in culture medium or blood.

In the structures we prepared, the walls are solid. Therefore, the oxygen permeability was determined using PTMC network films prepared by stereolithography in a similar manner 
as done for determination of the physical properties of the photo-crosslinked networks. Thinner films were used in this case, their thickness was $130 \pm 31 \mu \mathrm{m}$. At $37^{\circ} \mathrm{C}$ the oxygen permeability coefficient of the PTMC films was determined to be $0.55 \pm 0.19$ Barrer. The oxygen permeability of perfused tissues in vivo has not been determined, and values are derived by extrapolation of in vitro data using unperfused tissues. ${ }^{[39]}$ In this way, a value of 155 Barrer was determined for the oxygen permeability of muscle tissue.

By comparing this value with the one we determined for the solid PTMC networks, it is very likely that we will need to enhance the permeability of the channel walls before being able to successfully use such designed microvascular networks for the intended tissue engineering purposes (as seen in Figure 5). This will be done in work aimed at creating a network of interconnected pores in the walls of the channels. Resins will be formulated that after photo-crosslinking will form pores by phase separation methods involving extraction of a solvent in a non-solvent, or by extraction of leachable porogen components with nonsolvents. Using salt- or sugar particles sieved to specific dimensions, microporous crosslinked PTMC structures have been prepared previously. ${ }^{[40-41]}$ These micropores will not only allow the permeability of oxygen through the walls of the capillaries, but also of essential nutrients and metabolites.

\section{Conclusions}

A photo-crosslinkable resin based on a PTMC macromer with a molecular weight of $3150 \mathrm{~g} / \mathrm{mol}$ was formulated and used in stereolithography. Upon photo-crosslinking, the rubber-like PTMC networks showed good compatibility with cells. The networks allowed attachment of HUVECs already after $4 \mathrm{~h}$ of seeding and formed a near confluent layer after 3 days. A three-dimensional microvascular network with a high density of open capillary channels was designed and built at high resolution. The structures were flexible and elastic, and their dimensions closely matched the design. Imaging showed that the channels were open, and water could be perfused through the microvacular structure at physiological pressures. In the structures, the solid walls of the channels were not very permeable to oxygen. Structures with more permeable porous channel walls will need to be prepared before these designed PTMC structures can be used as perfusable tissue engineering scaffolds. 


\section{References}

[1] Organ Procurement and Transplantation Network (OPTN), Health Resources and Services Administraition (HRSA), U.S. Department of Health \& Human Services. http://optn.transplant.hrsa.gov/, accessed $13^{\text {th }}$ April 2011.

[2] Borenstein JT, Terai H, King KR, Weinberg EJ, Kaazempur-Mofrad MR, Vacanti JP. Microfabrication technology for vascularized tissue engineering. Biomed. Microdevices 2002, 4, 167-175.

[3] Kaihara S, Borenstein J, Koka R, Lalan S, Ochoa ER, Ravens M, Pien H, Cunninham $\mathrm{B}$, Vacanti JP. Silicon micromachining to tissue engineer branched vascular channels for liver fabrication. Tissue Eng. 2000, 6, 105-117.

[4] Ryu WH, Min SW, Hammerick KE, Vyakarnam M, Greco RS, Prinz FB, Fasching RJ. The construction of three-dimensional micro-fluidic scaffolds of biodegradable polymers by solvent vapor based bonding of micro-molded layers. Biomaterials 2007, 28, 1174-1184.

[5] King KR, Wang CJ, Kaazempur-Mofrad MR, Vacanti JP, Borenstein JT. Biodegradable microfluidics. Adv. Mater. 2004, 16, 2007-2012.

[6] Vozzi G, Flaim CJ, Ahluwalia A, Bhatia SN. Fabrication of PLGA scaffolds using soft lithography and microsyringe deposition. Biomaterials 2003, 24, 2533-2540.

[7] Bettinger CJ, Weinberg EJ, Wang Y, Borenstein JT, Langer R. Three-dimensional microfluidic tissue engineering scaffolds using a flexible biodegradable polymer. $A d v$. Mater. 2006, 18, 165-169.

[8] Folch A, Mezzour S, During M, Toner M, Muller R. Stacks of microfabricated structures as scaffolds for cell culture and tissue engineering. Biomed. Microdevices 2000, 2, 207-214.

[9] Borenstein JT, Weinberg EJ, Orrick BK, Sundback C, Kaazempur-Mofrad MR, Vacanti JP. Microfabrication of three-dimensional engineered scaffolds. Tissue Eng. 2007, 13, 1837-1844.

[10] Cooke MN, Fisher JP, Dean Dm, Rimnac C, Mikos AG. Use of stereolithography to manufacture critical-sized 3D biodegradable scaffolds for bone ingrowth. J. Biomed. Mater. Res. 2003, 64B, 65-69.

[11] Lee JW, Lan PX, Kim B, Lim G, Cho D-W. 3D scaffold fabrication with PPF/DEF using micro-stereolithography. Microelectron. Eng. 2007, 84, 1702-1705.

[12] Dhariwala B, Hunt E, Boland T. Rapid prototyping of tissue-engineerign constructs, using photopolymerizable hydrogels and stereolithography. Tissue Eng. 2004, 10, 1316-1322.

[13] Mapili G, Lu Y, Chen S, Roy K. Laser-layered microfabrication of spatially patterned functionalized tissue-engineering scaffolds. J. Biomed. Mater. Res. B 2005, 75B, 414424.

[14] Jansen J, Melchels FPW, Grijpma DW, Feijen J. Fumaric acid monoethyl esterfunctionalized poly(D,L-lactide)/N-vinyl-2-pyrrolidone resins for the preparation of tissue engineering scaffolds by stereolithography. Biomacromolecules 2009, 10, 214220.

[15] Melchels FPW, Feijen J, Grijpma DW. A poly(D,L-lactide) resin for the preparation of tissue engineering scaffolds by stereolithography. Biomaterials 2009, 30, 23-24.

[16] Matsuda T, Mizutani M. Liquid acrylate-endcapped biodegradable poly $(\varepsilon-$ caprolactone-co-trimethylene carbonate). II. Computer-aided stereolithographic microarchitectural surface photoconstructs. J. Biomed. Mater. Res. 2002, 62, 395-403. 
[17] Kwon IK, Matsuda T. Photo-polymerized microarchitectural constructs prepared by microstereolithography ( $\mu \mathrm{SL}$ ) using liquid acrylate-end-capped trimethylene carbonate-based prepolymers. Biomaterials 2005, 26, 1675-1684.

[18] Lee S-J, Kang H-W, Park JK, Rhie J-W, Hahn SK, Cho D-W. Application of microstereolithography in the development of three-dimenstional cartilage regeneration scaffolds. Biomed. Microdevices 2008, 10, 233-241.

[19] Chapter 6 of this thesis.

[20] Chapter 5 of this thesis.

[21] Fidkowski C, Kaazempur-Mofrad MR, Borenstein J, Vacanti JP, Langer R, Wand Y. Endothelialized microvascular based on a biodegradable elastomer. Tissue Eng. 2005, 11, 302-309.

[22] Lu Y, Mapili G, Suhali G, Chen SC, Roy K. A digital micro-mirror device-based system for the microfabrication of complex, spatially patterned tissue engineering scaffolds. J Biomed Mater Res 2006, 77A, 396-405.

[23] Melchels FPW, Feijen J, Grijpma DW. A review on stereolithography and its applications in biomedical engineering. Biomaterials 2010, 31, 6121-6130.

[24] Chapter 3 of this thesis.

[25] Bos A. PhD Thesis. High pressure $\mathrm{CO} 2 / \mathrm{CH} 4$ separation with glassy polymer membranes: Aspects of CO2-induced plasticization. $\mathrm{PhD}$ Thesis, University of Twente, 1996, Chapter 2.

[26] Sterescu DM, Stamatialis DF, Mendes E, Kruse J, Rätzke K, Faupel F, Wessling M. Boltorn-modified poly(2,6-dimethyl-1,4-phenylene oxide) gas separation membranes. Macromolecules 2007, 40, 5400-5410.

[27] Bat E, Kothman BHM, Higuera Sierra GA, van Blitterswijk CA, Feijen J, Grijpma DW. Ultraviolet light crosslinking of poly(trimethylene carbonate) for elastomeric tissue engineering scaffolds. Biomaterials 2010, 31, 8696-8705.

[28] Jacobs PF. Rapid Prototyping \& Manufacturing: Fundamentals of Stereolithography, $1^{\text {st }}$ edition; Society of Manufacturing Engineers (SME): Dearborn, MI, 1992.

[29] Seck TM, Melchels FPW, Feijen J, Grijpma DW. Designed biodegradable hydrogel structures prepared by stereolithography using poly(ethylene glycol)/poly(D,Llactide)-based resins. J. Controlled Release 2010, doi:10.1016/j.jconrel.2010.07.111

[30] Popel AS, Pittman RN. Mechanics and Transport in the Microcirculation, Chapter 31 in: The Biomedical Engineering Handbook, $2^{\text {nd }}$ edition; Bronzino JD, Ed.; CRC Press: Boca Raton, FL, 1999, p. 31-1 ff.

[31] Skalak TC, Price RJ. The role of mechanical stresses in microvascular remodeling. Microcirculation 1996, 3, 143-165.

[32] Cassell OC, Hofer SO, Morrison WA, Knight KR. Vascularisation of tissueengineered grafts: the regulation of angiogenesis in reconstructive surgery and in disease states. Br. J. Plast. Surg. 2002, 55, 603-610.

[33] Kannan RY, Salacinski HJ, Sales K, Butler P, Seifalian AM. The roles of tissue engineering and vascularisation in the development of micro-vascular networks: a review. Biomaterials 2005, 26, 1857-1875.

[34] Levick JR. An introduction to cardiovascular physiology, $3^{\text {rd }}$ edition; Arnold: London, 2000.

[35] Harrison RV, Harel N, Panesar J, Mount RJ. Blood capillary distribution correlates with hemodynamic-based functional imaging in cerebral cortex. Cerebral Cortex 2002, 12, 225-233.

[36] Melchels FPW, Bertoldi K, Gabbrielli R, Velders AH, Feijen J, Grijpma DW. Mathematically defined tissue engineering scaffold architectures prepared by stereolithography. Biomaterials 2010, 31, 6909-6916. 
[37] Bollinger A, Butti P, Barras J-P, Trachsler H, Siegenthaler W. Red blood cell velocity in nailfold capillaries of man measured by a television microscopy technique. Microvasc Res 1974, 7, 61-72.

[38] Ellis CG, Jagger J, Sharpe M. The microcirculation as a functional system. Critical Care 2005, 9, S3-S8.

[39] Dutta A, Popel AS. A theoretical analysis of intracellular oxygen diffusion. $J$ theor Biol 1995, 176, 433-445.

[40] Song Y, Wennink JWH, Kamphuis MMJ, Vermes I, Poot AA, Feijen J, Grijpma DW. Effective seeding of smooth muscle cells into tubular poly(trimethylene carbonate) scaffolds for vascular tissue engineering. J Biomed Mater Res A 2010, 95A, 440-446.

[41] Hou Q, Grijpma DW, Feijen J. Creep-resistant elastomeric networks prepared by photocrosslinking fumaric acid monoethyl ester-functionalized poly(trimethylene carbonate) oligomers. Acta Biomater 2009,5, 1543-1551.

[42] Computational Neurosciences Laboratory, Department of Biotechnology, Chennai, Tamil Nadu, India. http://www.biotech.iitm.ac.in/faculty/annlabweb/publication.htm, accessed $13^{\text {th }}$ April 2011. 


\section{Summary}

There is an evident need in the biomedical field to develop (new) materials with tailored properties for specific applications. In addition it is essential to be able to process these materials into desired two- and three-dimensional shapes and (micro)structures. Microfabrication, and especially soft lithography, allows the creation of two-dimensional microstructures with features having size scales that match the dimensions of microorganisms and of individual cells of more complex organisms. Using twodimensional microstructured surfaces, investigations in the biomedical field involving cells, proteins and other biomolecules have been successfully conducted. Examples include microfluidics-based biological systems, microarrays and tissue engineering applications. Tissue engineering requires a three-dimensional scaffold, and multiple layers of two-dimensional microstructures have been stacked to realize this. However, significantly more reproducible three-dimensional structures can be obtained by stereolithography. This rapid prototyping technique allows creating designed scaffolding structures at high resolutions of upto $20 \mu \mathrm{m}$. Unfortunately, most resins available for stereolithography do not yield the flexible and elastic biodegradable network materials that are required for the engineering of soft tissues or organs.

This thesis describes the development of poly(trimethylene carbonate) (PTMC) macromers, their formulation into photo-crosslinkable resins and their processing into designed flexible and elastic creep-resistant structures.

Chapter 1 briefly describes the aim and outline of this thesis. Chapter 2 provides the fundamental background on microfabrication and stereolithography, focussing on important applications in the biomedical field. Furthermore, the properties and applications of PTMC are reviewed. The mechanical performance of linear PTMC strongly depends on the molecular weight of the polymer. In this thesis, the mechanical properties of a variety of PTMC networks prepared by photo-crosslinking have been investigated. A series of threearmed methacrylate-functionalized polytrimethylene carbonate macromers with number average molecular weights $\left(\overline{\mathrm{M}}_{n}\right)$ between 1000 and $41000 \mathrm{~g} / \mathrm{mol}$ was synthesized and photo-crosslinked either in the melt (Chapter 3) or in presence of a non-reactive diluent (Chapter 6). In Chapter 3 the mechanical properties of the PTMC networks photo-crosslinked in the melt are described. It was found that all PTMC networks were 
highly resistant to creep. Their tensile properties, toughness and the tearing behaviour were strongly influenced by the $\overline{\mathrm{M}}_{n}$ of the macromer. Low molecular weight PTMC macromers yield relatively stiff networks with low elongations at break. With increasing $\overline{\mathrm{M}}_{n}$, the elastic modulus of the networks decreases to a value of approximately $5 \mathrm{MPa}$, while the elongation at break increased to more than $1200 \%$. The tensile strength and the suture retention strength (and tear resistance) increase with macromer molecular weight at $\overline{\mathrm{M}}_{n}$ values higher than $10000 \mathrm{~g} / \mathrm{mol}$. These networks are tough, and show yielding behavior and rubber plateaus in tensile testing. The mechanical properties of the PTMC networks were compared to those of well-known elastomers like PDMS and latex rubber, as well as to those of native blood vessels.

Chapter 4 describes the application of photo-crosslinkable PTMC macromers in microfabrication as a biodegradable alternative to PDMS. Within minutes two-dimensional microstructured surfaces could be obtained by hot embossing PTMC resins against a structured master and subsequent photo-crosslinking. The shrinkage of the network structures was less than $5.7 \%$, and the fidelity of the replicated features was comparable to that obtained using PDMS. Processing PTMC resins in this way is just as facile as processing PDMS, but significantly faster. This enables application of the resins in high-throughput processes. Microstructured PTMC was investigated for use in two different applications: as stamps to transfer a model protein to another surface and as structured substrate for cell culture. In the stamping process, the protein was successfully transferred at high resolutions from the PTMC microstructure to a glass surface. In cell culturing of mesenchymal stem cells (MSC), photo-crosslinked PTMC was found to be compatible with cells, showing better cell adhesion after 1 day of culture than PDMS. MSCs proliferated well on both smooth and on microstructured PTMC surfaces for periods of up to 7 days. Although these processes remain to be optimized, photo-crosslinked PTMC seems to be an excellent alternative to PDMS in life science and medical applications, especially when biodegradation of the structures is required.

In view of the results obtained in Chapter 3, the application of PTMC resins based on macromers of molecular weights between 3250 and $27750 \mathrm{~g} / \mathrm{mol}$ in the stereolithography apparatus is described in Chapter 5. Photo-crosslinkable resins containing the PTMC macromers, propylene carbonate as non-reactive diluent, a photo-initiator and a dye were formulated and processed by stereolithography at temperatures between room temperature and $65{ }^{\circ} \mathrm{C}$. The network films prepared from these resins were flexible with E-moduli of 
approximately $3.8 \mathrm{MPa}$ and elongations at break up to nearly $1000 \%$. Designed solid cylindrical structures and porous cylindrical structures with a gyroid pore network architecture could readily be built from PTMC resins containing up to $45 \mathrm{wt} \%$ diluent and from formulations in which the macromer molecular weights were less than $20150 \mathrm{~g} / \mathrm{mol}$. The average pore sizes and porosities of the porous structures built, however, were somewhat lower than designed. In compression tests the porous structures (porosities between 53 and 66\%) showed compression moduli between 156 and $20 \mathrm{kPa}$. These values are much lower compared to the ones obtained for the solid structures, being between 3.1 and 4.2 MPa.

Chapter 6 describes PTMC structures prepared by stereolithography using macromers with $\overline{\mathbf{M}}_{n}=3100 \mathrm{~g} / \mathrm{mol}$ and their applicability as scaffolds in cartilage tissue engineering. Bovine chondrocytes, seeded in the designed scaffolds with average pore sizes of $350 \pm 12 \mu \mathrm{m}$ and porosities of $54 \pm 2.2 \%$ adhered well and spread on the PTMC surface. After 6 weeks of culturing, cells showing a round morphology, indicative of the differentiated chondrocyte phenotype, were also present and adhered to the layer of spread cells. Cells deposited sulphated glycosaminoglycans and fibrillar collagen. Comparison of the initial compression modulus of the structure with that after 6 weeks of culture showed an increase of $50 \%$ to a value of approximately $100 \mathrm{kPa}$.

The design and fabrication of a three-dimensional microvascular network is described in Chapter 7. In the design, open channels branch into 5 generations of 4 smaller channels and reunite again in the same way. The smallest channels mimic densely arranged capillaries. The overall open cross-sectional area remains constant throughout the scaffold, allowing medium or blood to be perfused through the scaffold. Using this design in stereolithography, the structure was built using a resin based on a PTMC macromer with molecular weight of $3150 \mathrm{~g} / \mathrm{mol}$. This resin was processed in the absence of a diluent at 60 ${ }^{\circ} \mathrm{C}$. The obtained structures were flexible and elastic, with open square cross-sectional areas allowing water to perfuse at physiological pressures. The smallest channels had inner widths of approximately $224 \mu \mathrm{m}$ and wall thicknesses of approximately $152 \mu \mathrm{m}$. HUVECs were seeded on the surface of flat disks prepared from the photo-crosslinked resin and adhered and proliferated well.

The work described in this thesis demonstrates that photo-crosslinkable PTMC can be easily synthesized and processed by microfabrication and stereolithography techniques. The mechanical characteristics of the networks obtained by photo-crosslinking PTMC 
macromers depend on the molecular weight of the macromers used. Initial cell culturing experiments on flat surfaces, two-dimensional microstructured surfaces and porous threedimensional scaffolds prepared by stereolithography confirmed the biocompatibility of the obtained materials. However, there still remain challenges that need to be solved. Concerning tissue engineering, the creation of three-dimensional microvascular structures with porous walls is critical. Also, experiments to optimize cell expansion in and on structures prepared by stereolithography need to be carried out. 


\section{Samenvatting}

In de biomedische technologie bestaat een uitgesproken behoefte aan de ontwikkeling van nieuwe materialen met bijzondere eigenschappen voor specifieke toepassingen. Bovendien is het noodzakelijk dat deze materialen in twee- en driedimensionale vormen en (micro)structuren verwerkt kunnen worden. Microfabricagetechnieken, in het bijzonder de zogenaamde ,zachte“ lithografie (eng. soft lithography), stellen ons in staat om tweedimensionale structuren op de schaal van micro-organismen en cellen te maken. Met behulp van zulke tweedimensionale, microgestructureerde oppervlakken werden al diverse biomedische onderzoeken aan cellen, eiwitten en andere biomoleculen met succes uitgevoerd. Onder andere werden op deze manier microfluidische systemen, microarrays en dragermaterialen voor weefselkweek (eng. tissue engineering) ontwikkeld. Voor het kweken van een complex weefsel of orgaan is een driedimensionale raamstructuur (eng. scaffold) nodig. Een bruikbare driedimensionale structuur kan verkregen worden door het opeenstapelen van tweedimensionale microstructuren. Echter, een dergelijke driedimensionale structuur kan veel beter verkregen worden met behulp van stereolithografie. Stereolithografie is een snelle methode voor het maken van prototypes (eng. rapid prototyping) met microstructuren van ongeveer 20 micron. Helaas resulteren de gangbare harsen voor stereolithografie in harde en breekbare structuren in plaats van buigzame, elastische en biologisch afbreekbare materialen zoals die voor het kweken van weefsels en organen nodig zijn.

Dit proefschrift beschrijft de ontwikkeling van poly(trimethyleen carbonaat) (PTMC) macromeren, de verwerking van PTMC macromeren in harsen die onder belichting uitharden tot netwerkpolymeren, en het bereiden van buigzame en elastische PTMC microstructuren met hoge kruipweerstand.

Hoofdstuk 1 is een korte uiteenzetting van het doel en de opzet van het proefschrift.

Hoofdstuk 2 beschrijft de stand van kennis op het gebied van microfabricage en stereolithografie, met nadruk op belangrijke toepassingen in de biomedische technologie. Bovendien worden de eigenschappen en toepassingen van PTMC besproken. De mechanische eigenschappen van lineair PTMC worden bepaald door het molecuulgewicht van het polymeer. In dit proefschrift werden de eigenschappen van diverse PTMC netwerkpolymeren onderzocht, die door belichting van PTMC macromeren ontstaan. 
Hiertoe werd een serie drie-armige methacrylaat-gefunctionaliseerde PTMC macromeren met aantalgemiddeld molecuulgewicht $\left(\overline{\mathrm{M}}_{n}\right)$ tussen 1000 en $41000 \mathrm{~g} / \mathrm{mol}$ gesynthetiseerd en door belichting uitgehard, hetzij in onverdunde toestand (Hoofdstuk 3), hetzij in aanwezigheid van een inerte verdunner (Hoofdstuk 6). In Hoofdstuk 3 worden de mechanische eigenschappen van PTMC netwerkpolymeren beschreven, die werden verkregen door belichting in onverdunde toestand. Alle PTMC netwerkpolymeren hebben een hoge kruipweerstand. De treksterkte en de elasticiteit zijn sterk afhankelijk van $\overline{\mathrm{M}}_{n}$ van het gebruikte macromeer. PTMC macromeren met een laag molecuulgewicht resulteren in stijve netwerkpolymeren met een geringe rek voordat breuk optreedt. Bij hogere $\overline{\mathrm{M}}_{n}$ neemt de elasticiteitsmodulus van het netwerkpolymeer af tot ongeveer $5 \mathrm{MPa}$, terwijl de maximale rek toeneemt tot meer dan $1200 \%$. De treksterkte en de hechtsterkte (en ook de trekweerstand) nemen weer toe met het molecuulgewicht van het macromeer bij $\overline{\mathrm{M}}_{n}$ waarden groter dan $10000 \mathrm{~g} / \mathrm{mol}$. Deze netwerkpolymeren zijn sterk en vertonen rubber plateaus in de trekproeven. De mechanische eigenschappen van de PTMC netwerkpolymeren worden vergeleken met de eigenschappen van bekende elastomeren zoals poly(dimethylsiloxaan) (PDMS) en latex rubber, evenals met de eigenschappen van natuurlijke bloedvaten.

Hoofdstuk 4 behandelt de toepassing van PTMC in microfabricage als biologisch afbreekbaar alternatief voor PDMS. Tweedimensionale microgestructureerde oppervlakken werden binnen enkele minuten verkregen door het indrukken (eng. hot embossing) van een dunne laag PTMC hars met een microgestructureerde stempel, gevolgd door belichting en uitharden. De krimp van de netwerkpolymeer structuren bedroeg minder dan 5.7\%, en de nauwkeurigheid van de microstructuren was vergelijkbaar met structuren van PDMS. Het structureren van PTMC harsen is op deze manier net zo eenvoudig als het structureren van PDMS, maar verloopt zeer veel sneller. De PTMC harsen kunnen dus desgewenst in zeer snelle fabricage processen (eng. high-throughput processing) ingezet worden. De PTMC microstructuren werden met het oog op twee toepassingen onderzocht: als stempels om eiwitten in microstructuren op oppervlakken aan te brengen en als gestructureerde ondergrond voor het kweken van cellen. Tijdens het stempelen werd het eiwit inderdaad met grote nauwkeurigheid van de PTMC stempel op een glasoppervlak overgedragen. In een celkweek van mesenchymale stamcellen (MSC) werd gevonden dat cellen het PTMC netwerkpolymeer goed verdragen en na 1 dag beter aan PTMC hechten dan aan PDMS. De MSC groeien en delen op gladde en op microgestructureerde PTMC oppervlakken 
gedurende 7 dagen. Alhoewel deze processen verder geoptimaliseerd moeten worden, tonen deze experimenten aan dat PTMC netwerkpolymeren een uitstekend alternatief voor PDMS kunnen zijn voor toepassing in de levenswetenschappen en de biomedische technologie, met name wanneer de polymeerstructuren biologisch afbreekbaar moeten zijn.

In aansluiting op de resultaten beschreven in Hoofdstuk 3, wordt in Hoofdstuk 5 de toepassing van PTMC macromeren met een molecuulgewicht tussen 3250 en 27750 g/mol in de stereolithografie onderzocht. Een vloeibare hars bestaande uit een PTMC macromeer, trimethyleen carbonaat als inerte verdunner, een foto-initiator en een kleurstof werd in de stereolithografie-opstelling uitgehard bij een temperatuur tussen kamertemperatuur en $65^{\circ} \mathrm{C}$. Op deze manier werden dunne films van netwerkpolymeren verkregen met een elasticiteitmodulus van ongeveer 3.8 MPa en maximale rek van bijna 1000\%. Ook was het mogelijk om gevulde cilindrische structuren en gyroidale netwerken van holle cilinders te maken. Het bleek dat het voor stereolithografie noodzakelijk is dat de PTMC harsen tot 45 gewichtsprocent verdunner bevatten, tenzij het PTMC macromeer een molecuulgewicht lager dan $20150 \mathrm{~g} / \mathrm{mol}$ heeft. De gemiddelde poriegrootte en de porositeit van de verkregen structuren waren iets geringer dan voorzien. In compressietesten werd voor de poreuze structuren (met een porositeit tussen 53 en $66 \%$ ) een compressiemodulus tussen 156 en $20 \mathrm{kPa}$ gemeten. Deze waarden zijn zeer veel lager dan de compressiemodulus voor de gevulde structuren (3.1 tot $4.2 \mathrm{MPa})$.

Hoofdstuk 6 beschrijft PTMC structuren gemaakt door stereolithografie met macromeren met $\overline{\mathrm{M}}_{n}=3100 \mathrm{~g} / \mathrm{mol}$ en hun toepassing als dragermateriaal voor het kweken van kraakbeenweefsel. Runder chondrocyten werden in een PTMC dragerstructuur met een gemiddelde poriegrootte van $350 \pm 12 \mu \mathrm{m}$ en een porositeit van $54 \pm 2.2 \%$ gezaaid. De cellen hechtten goed aan het PTMC oppervlak en verspreidden zich over de gehele structuur. Na 6 weken hadden veel cellen een typische ronde vorm, die duidt op het gedifferentieerde chondrocyte phenotype. Deze cellen werden bevonden zich op een onderlaag van cellen die aan het PTMC oppervlak hechten. De cellen makten gesulfateerde glycosaminoglycanen en collageen vezels aan. Een vergelijking van de compressiemodulus voor en na de celkweek toonde aan dat de modulus met $50 \%$ toenam tot een waarde van ongeveer $100 \mathrm{kPa}$.

Het ontwerp en het maken van driedimensionale microcapillaire netwerken wordt beschreven in Hoofdstuk 7. In dit ontwerp vertakt zich een open kanaal in 5 opeenvolgende stappen tot in totaal 256 nauwe kanaaltjes. Daarna komen de kanaaltjes op 
dezelfde stapsgewijze manier weer samen. Deze bundeling van kleinste kanaaltjes is een goed model voor microcapillaire bloedvaten. De totale diameter van de kanalen blijft constant, zodat een vloeibare stof (bijvoorbeeld bloed) door de structuur kan stromen. Door middel van stereolithografie werd dit ontwerp - uitgaande van een hars van een PTMC macromeer met een molecuulgewicht van $3150 \mathrm{~g} / \mathrm{mol}$ - in een driedimensionale netwerkstructuur gerealiseerd. De hars werd zonder verdunner bij $60{ }^{\circ} \mathrm{C}$ verwerkt. De verkregen structuur was buigzaam en elastisch, met open kanalen die de doorstroom van water bij een fysiologische druk toelieten. De kleinste kanaaltjes hadden een interne diameter van ongeveer 224 micron en een wanddikte van ongeveer 152 micron. Menselijke navelstreng endotheel cellen (HUVEC) werden op een dunne film van hetzelfde netwerkpolymeer uitgezaaid en hechten en groeiden daar goed op.

De experimenten beschreven in dit proefschrift tonen aan dat PTMC macromeren op eenvoudige wijze gesynthetiseerd kunnen worden en toegepast in microfabricage en stereolithografie. De mechanische eigenschappen van de netwerkpolymeren die werden verkregen door belichting van de PTMC macromeren worden bepaald door het molecuulgewicht van het macromeer. Eerste celkweekexperimenten op gladde oppervlakken, gemicrostructureerde oppervlakken en complexe, poreuze driedimensionale structuren bevestigen de biocompatibiliteit van de netwerkpolymeren. Desondanks blijven er een aantal uitdagingen voor vervolgonderzoek. Voor daadwerkelijke toepassing in de kweek van kunstmatige weefsels is het noodzakelijk dat de microcapillaire netwerken een poreuze wand hebben. Ook zal de groei van cellen op de dragerstructuren verbeterd moeten worden. 


\section{Acknowledgements}

It is great to finally write this part of the thesis. Although my $\mathrm{PhD}$ did not always go as smoothly as I hoped in the beginning, I enjoyed the last six years. For me, not only work itself is important, but especially the environment and in particular the people I deal with. At the UT I met great colleagues, and some of them have become close friends. Now the time has come to acknowledge them and all others who participated somehow to this thesis. First of all, I want to thank my promotors Jan Feijen and Dirk Grijpma for their trust and support during the years, as well as for their understanding in difficult times. Jan Feijen, I am grateful that you gave me the opportunity to pursue my $\mathrm{PhD}$ in your group. I always appreciated your honesty, friendliness, and input in my work. Dirk, thank you for bringing me back on the right path when I got lost in details and couldn't see the forest for the trees anymore. I often remembered what you said to me once: KISS. Keep It Simple Stupid! I have learned a lot from you at work. Additionally, in Argentinean Tango, I had the pleasure of being (literally) one step ahead of you!

I want to thank the members of my promotion committee: Professor David Reinhoudt, Professor Leo Koole, Professor Wilma Dierkes, Professor Clemens van Blitterswijk, and Professor Matthias Wessling, for reading and approving my thesis.

I would also like to express my gratitude to the scientific staff members of the former PBM group. André Poot, thank you very much for the discussions about the work of Chapter 6 and for supporting me in the writing process on very short notice. I would also like to acknowledge Piet Dijkstra for encouraging me at the right moments and Johan Engbersen for nice conversations.

'Behind every great man there stands a great woman'. In case of (former) PBM, this quote can be derived to: 'behind every great group there stands a great secretary'. Karin, next to performing your tasks as secretary to the staff, you have always been there to support the rest of the group. Thank you very much for all your help (in a variety of things), your interest in me and my family and our great conversations! Also you, Hetty, deserve many words of gratitude! You did not only provide me with useful tips regarding work related stuff, but also in questions of how to educate a child! Thank you for placing Elisabeth as babysitter! Another person I want to deeply thank is Anita (Podt). I am grateful for your support, especially during the last year. You were always willing to start a build job, a $\mu \mathrm{CT}$ scan, to change solvent, etc., so I could leave and pick up Jesse on time from daycare. You 
are an angel! Zlata, without you I wouldn't have been able to do chemistry! It did not matter what I needed - chemicals, equipment, a new bottle of nitrogen, or anything else - I knew you would help straight away! Thank you very much! Marc, lab safety has been one of your main duties and you have been very fussy (and rightly so!) in that regard. I am thankful you 'adopted' me (for a while) when going for lunch to the Bastille. With that you assured my physical safety, as I otherwise (lacking time for preparing lunch at home) might have eaten unhealthy deep-fried stuff in front of the computer.

Now it is time to thank 'my' students, which I supervised during their bachelor or master projects. Talitha, you have been the first student I supervised. I enjoyed the months we worked together, especially because we had the same style of working. Anne, you started to become more enthusiastic about your project when due to my hand injury you could try more things in the lab without me watching and we became friends. I always liked your lively character, and in the end we became friends. Erwin, we have worked together for 11 months and I owe you my gratitude for laying the foundation for Chapter 7. You became acquainted with Rhino and designed the nice structure of 'our' microvascular network and other fancy pictures which are displayed in this thesis. Additionally, you learned to deal with the stereolithography apparatus and transferred your experience to me. The nice PTMC structure that we were finally able to build is partly due to your efforts.

Sandra Teixeira, you performed the cell experiments as post-doc on the project. Thank you for your input to Chapters 4, 6 and 7.

In the following paragraphs I want to acknowledge my dear colleagues which I dealt with on a daily basis. First of all, I want to thank Henriette (Jet), Erhan, and Ferry, which turned from colleagues into great friends on which I could ALWAYS rely! Together with Willeke, Arzu, and Rob we often met for dinner and to play games. I am grateful to all of you for the 'gezelligheid'. Jet, although you decided to follow another road we never lost contact and met regularly. Thank you for being there at all times, and especially for staying by my side as paranimf. Erhan and Ferry, first we shared the lab, later I was happy to also call you 'office mates'. We talked about everything and have always been there for each other, helping in word and deed. Thank you both for your advice, help, candidness, honesty, and friendship! Erhan, my dear paranimf, you are one of the nicest persons I have met in my life (and a very reliable source regarding PTMC literature)! Ferry, although I sometimes asked you 'stupid' questions you never minded answering. And although you are at the other side of the world we are in touch with each other. 
Continuing with my lab mates I want to thank Janine. It has been a pleasure to work with you in a lab (although being the lab chief you might not have always been happy with my style of working). I always envied your ability to organize your work and be finished on time (or even before!); I still would "like to be a little bit more Janine"! Sameer, little Indian fellow: when you entered the lab it became lively and happy! Thank you for cooking 'real' Indian food and for trying to teach me the 'moonwalk'. By the way: you have been the best 'Zwarte Piet' I have ever seen! Zheng, thank you for helping me in the lab during the first weeks of my $\mathrm{PhD}$ and for helpful discussions about PTMC. Alfredo, thank you for all the talks and discussions we had about work related topics and private things, in particular Italian food.

There are some things for which I want to acknowledge all (former) members of the PBM, BST and BMC groups: thank all of you for the 'gezelligheid' at the coffee table, borrels, parties, conferences, or other events. Regarding work I want to acknowledge you for being helpful when I had cut the tendon of my right hand, becoming a bit disabled for a while, and for assisting me in the lab during my pregnancy. Niels, especially in the last mentioned period you have been a great support to me, helping me out where you could (e.g. filling the heavy DSC nitrogen tank -without me even asking!). Also, thank you for your support during the movie making process for e.g. Erhan and Ferry (as actor and advisor)! Hans, you are a great actor! I would have never dared to play air-guitar in front of ACTION on a Thursday evening! Thanks for all the fun and laughter, as well as for (co)organizing all kind of borrels. Although I needed to get used to your way of talking German, I appreciated that you just did! Sytze, you were my favourite (male) neighbour! Thanks for babysitting Jesse once! I am sure you will never forget that experience and maybe neither will he $:$. Jos, I want to thank you for your cooperation, especially during your master project. Regarding your roles in the PBM / BST / BMC movies I am wondering if you will also turn out to be a professor... Ingrid, on the first day as a $\mathrm{PhD}$ student you picked me up for lunch. I appreciated this a lot and I am glad we became friends! Together with Christine and Mark (ten Breteler), you taught me the Dutch language! Marloes, you are such a lovely person! Whenever you appeared there was liveliness in the air that was infectious! I am glad we are still in touch although you are in Australia! Woika, you are also a woman with a lot of vitality! Thanks for being a friend! Martin, you turned around a day that started crappy when you spontaneously fixed the tire of my bike! Thanks! Andries, although we do not always have the same type of humour, we did always get along very well. Especially when 
going out partying, I enjoyed dancing with you! Kim, thanks for introducing me to the cell lab and for "kicking" Andries at the right moments ;-). Gregory, thank you for organizing all kinds of parties! You are a great chef and baker! Anita (Krouwels), you have been in the group for less than one year but we still are in touch! Let's keep it that way! Shahriar, thanks for fruitful discussions about PTMC and other work related topics! Jung Seok, thanks for the nice Korean dinner at your place. Lanti, I will never forget the great taste of the Coca-Cola chicken! Boon Hua, you have been my first office mate. It's a pity you left the office to become a member of the 'kippenhok'! Arkadiy, endlich einer der 'richtig' Deutsch gesprochen hat (nicht im 'Militär'-Stil). Danke für die gemütlichen Stunden mit dir, Olga, Sasha, und Yuri! Bas (Siebum), thank you for the nice conversations at work and the great evenings at "de Beiaard". Federica, it's a pity you left our group. I enjoyed the high-teas we had together with Jet between Randstad and Enschede! Finally, I want to thank all other (former) PBM members that have not been mentioned above by name. Thanks for the great atmosphere in the group!

From Medisse I want to thank Sandra (de Vos), Vincent and Kasia. Sandra, it has always been great to talk to you about many things, but especially about being a working mother. Thank you very much for your good advice and for cheering me up! Vincent and Kasia, we shared the office only for a few months and weeks, but it has been a friendly and warm atmosphere, thanks for that!

I also want to thank the collaborators in the "Advanced Polymeric Microstructures for Tissue Engineering” program: Bernke and Dimitrios (at that time in the Membrane Science and Technology group), as well as Tomasz and Hans (Nanobiophysics group). Thank you for interesting and constructive meetings, discussions, and experiments.

Furthermore, from the Membrane Science and Technology group I want to thank Jeroen (Ploegmakers) for measuring the oxygen permeability of my PTMC films (Chapter 7). Mark Smithers (Mesa+), thank you very much for performing SEM measurements on short notice when "our" machine was broken! Also another apparatus was "on strike" when I needed to finish my experimental work: our $\mu \mathrm{CT}$. I want to thank Professor Frauke Alves of the Universitätsklinikum Göttingen for allowing me to accomplish my last measurements with their machine. Dr. Jeannine Mißbach-Güntner and Christian Dullin: "Vielen Dank" for the warm welcome in Göttingen and for your assistance during these days!

Each $\mathrm{PhD}$-thesis is unique, just as its cover. Ingeborg, thank you so much for making my thesis special by designing and painting the cover! I am glad you have also drawn the 
scheme (Figure 5) in Chapter 7 as it is just as I wanted it to be, but couldn't get it like that myself!

During the time when PBM was located in "Langezijds", I socialized with people from SMCT, MTP and ETE (former RBT). Although it is impossible to name all, I want to thank Aldrik and Bianca for their help with questions regarding NMR; Clemens (Padberg) for helping with all kind of technical matters; Jacob for being supportive not only in work related topics, but also in personal issues. Monique, Nina (it has been a pleasure to be your paranimf!), Janet, Oya, Eddy, Denis, Thomas, Marina, Miguel, Debby, Anne Corine, Rani, Arun, Debassis, Mukund, Rui, Francesca, Montse and all other (former) members of MTP, STEP and ETE: thank you for the great coffee breaks, borrels, and unforgettable parties we enjoyed together!

Now the time has come to thank my family and friends, close by and far away. Because you have always supported me and believed in me I dedicate this thesis to all of you!

Let me start with my friends. I cannot mention all of you by name (some I did already), but to name a few: Yuko and Emma: thank you very much for babysitting or playing with Jesse when I needed to go to the lab in evenings or weekends; Jurriaan, I really appreciated your comments on Chapter 4; Kerstin, Vroni and Quirin: I am very grateful to have friends like you. It is sometimes so much easier to tell things in your own language. Thank you for always listening and advising me! Monique, thank you for reading through and correcting parts of my thesis. From the beginning (at the coffee corner) we have been on the same wavelength and although there are thousands of kilometers between us we share a deep friendship.

Finally the last words are for my family. Bart Jan, the last years haven't been always easy. Nevertheless you stood by me, encouraged and supported me at all times. Thank you for your love and patience (especially during the last year). Ich liebe Dich und unseren kleinen „Boef“! Jesse, auch wenn du noch nicht lesen kannst, möchte ich dir für all' die Küsschen und Umarmungen danken die ich in den letzten 3 Jahren von dir bekommen habe. Hoffentlich hörst du nie damit auf! Mama und Karin, ich wünschte Papa hätte das Ende der Doktorarbeit auch noch miterlebt! Vielen Dank für eure Liebe und Unterstützung! Lieve schoonfamilie, ook jullie wil ik van harte bedanken voor de steun in de afgelopen jaren. Jullie zijn altijd geïnteresseerd in mijn werk en hoe het met mij gaat. Eindelijk is het nu zover. Het proefschrift is af en ik heb weer tijd voor de leukere dingen in het leven. Ik zal weer regelmatig lekkere taarten voor jullie bakken $\odot$ !

\section{Siggi}




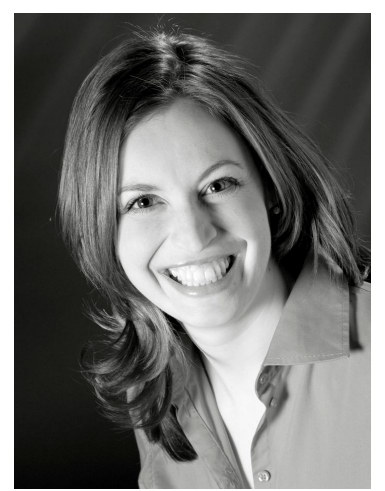

\section{Curriculum Vitae}

Sigrid Schüller-Ravoo was born on $15^{\text {th }}$ of May 1977 in Sibiu (Romania) and grew up in Ingolstadt (Germany). After completing an apprenticeship as Electronics Technician at Audi AG, she went back to school to obtain her diploma, enabling her to enter University. Between 1998 and 2004 she studied Chemistry at the University of Regensburg. She completed her study with the diploma thesis "Novel Surfactants for Separation and Environmental Applications: Aluminium Soaps - Synthesis, Characterisation and Application" in the research group of Prof. Dr. Werner Kunz. During her study she spent one semester at University College Dublin (Ireland), where she was involved in a project regarding the microwave parallel synthesis of substituted 4(5)-sulfanyl-1H-imidazole libraries in the research group of Prof. Dr. Donal O'Shea. In March 2005 she joined the research group Polymer Chemistry and Biomaterials of Prof. Dr. Jan Feijen at the University of Twente, Enschede (The Netherlands). Her research was supervised by Prof. Dr. Dirk W. Grijpma and the results are reported in this thesis. Currently she is working at European Research Services GmbH in Münster. 
